Portland State University

PDXScholar

Civil and Environmental Engineering Faculty

Publications and Presentations

Civil and Environmental Engineering

$7-2015$

\title{
Bridge Seismic Retrofit Measures Considering Subduction Zone Earthquakes
}

\author{
Peter Dusicka \\ Portland State University \\ Ramiro Bazaez \\ Portland State University \\ Sarah Knoles \\ Portland State University
}

Follow this and additional works at: https://pdxscholar.library.pdx.edu/cengin_fac

Part of the Civil Engineering Commons, and the Structural Engineering Commons

Let us know how access to this document benefits you.

\section{Citation Details}

Dusicka, P., Bazaez, R., and Knoles, S. (2015). Bridge Seismic Retrofit Measures Considering Subduction Zone Earthquakes. Report FHWA-OR-RD-16-01. https://doi.org/10.15760/trec.144

This Report is brought to you for free and open access. It has been accepted for inclusion in Civil and Environmental Engineering Faculty Publications and Presentations by an authorized administrator of PDXScholar. Please contact us if we can make this document more accessible: pdxscholar@pdx.edu. 


\section{BRIDGE SEISMIC RETROFIT MEASURES CONSIDERING SUBDUCTION ZONE EARTHQUAKES}

Final Report

SPR 741

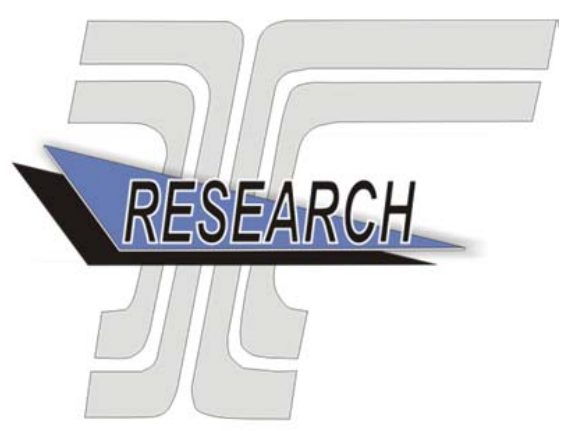





\title{
BRIDGE SEISMIC RETROFIT MEASURES CONSIDERING SUBDUCTION ZONE EARTHQUAKES
}

\section{Final Report}

\section{SPR 741}

\author{
By \\ Peter Dusicka \\ Associate Professor \\ Ramiro Bazaez \\ Graduate Research Assistant \\ Sarah Knoles \\ Former Graduate Research Assistant \\ Portland State University \\ for \\ Oregon Department of Transportation \\ Research Section \\ $55513^{\text {th }}$ Street NE, Suite 1 \\ Salem OR 97301 \\ and \\ Federal Highway Administration \\ 400 Seventh Street, SW \\ Washington, DC 20590-0003
}

July 2015 

Technical Report Documentation Page

\begin{tabular}{l|l|l}
$\begin{array}{l}\text { 1. Report No. } \\
\text { FHWA-OR-RD-16-01 }\end{array}$ & 2. Government Accession No. & 3. Recipient's Catalog No.
\end{tabular}

\section{Title and Subtitle}

Bridge Seismic Retrofit Measures Considering Subduction Zone Earthquakes
5. Report Date

-July 2015-

6. Performing Organization Code

8. Performing Organization Report No.

SPR 741

9. Performing Organization Name and Address

Department of Civil \& Environmental Engineering

Portland State University

1930 SW 4th Avenue, Suite 200

10. Work Unit No. (TRAIS)

Portland, Oregon 97201

12. Sponsoring Agency Name and Address

Oregon Dept. of Transportation

Research Section

$55513^{\text {th }}$ Street NE, Suite 1

and Federal Highway Admin.

400 Seventh Street, SW

Salem, OR 97301

Washington, DC 20590-0003

11. Contract or Grant No.

\section{Supplementary Notes}

16. Abstract: Over the years, earthquakes have exposed the vulnerability of reinforced concrete structures under seismic loads. The recent occurrence of highly devastating earthquakes near instrumented regions, e.g. 2010 Maule, Chile and 2011 Tohoku, Japan, has demonstrated the catastrophic impact of such natural force upon reinforced concrete structures. Research was conducted to investigate the effect of subduction zone earthquakes on structural damage. The study suggests that large magnitude ground motions of long duration have the potential of significantly increasing the number of inelastic excursions and consequently incur more extensive structural damage as compared to ground motions with similar elastic spectral demands but of shorter duration. This increase in demand plays a crucial role in the Pacific Northwest where a mega subduction zone earthquake is impending.

Typical reinforced concrete bridge bents constructed in the 1950 to mid-1970 in the State of Oregon were designed and built with minimum seismic considerations. This resulted in inadequate detailing within plastic hinge zones, leaving numerous RC bents highly susceptible to damage following an earthquake. In this study, the cyclic performance of an asbuilt RC square column and a reinforced concrete bridge bent retrofitted using buckling restrained braces (BRBs) was experimentally evaluated using quasi-static cyclic loading protocols aiming to reflect subduction zone earthquake demands up to displacement ductility. The buckling restrained braces were designed as replaceable elements in order to take the earthquake-induced energy and dissipate it through nonlinear hysteretic behavior. Two BRB designs were considered in the study in an effort to assess the influence of BRB stiffness on the overall structural performance. The results of these large-scale experiments successfully demonstrated the effectiveness of utilizing buckling restrained braces for achieving high displacement ductility of the retrofitted structure, while also controlling the damage of the existing vulnerable reinforced concrete bent up to the design performance levels. The potential of improving the overall seismic behavior and the design performance levels with BRBs offers structural design professionals a viable method for performance driven retrofit of reinforced concrete bents.

17.Key Words

Subduction zone earthquakes, reinforced concrete bridge bents, plastic hinge zone, performance levels, bridge seismic retrofit, 18.Distribution Statement: Copies available from NTIS, and online at http://www.oregon.gov/ODOT/TD/TP_RES/ quasi-static loading protocol, buckling restrained braces.

\begin{tabular}{l|c}
$\begin{array}{l}\text { 19. Security Classification (of this } \\
\text { report) }\end{array}$ & $\begin{array}{l}\text { 20. Security Classification (of this } \\
\text { page) } \\
\text { Unclassified }\end{array}$ \\
\hline
\end{tabular}

Technical Report Form DOT F 1700.7 (8-72)
21. No. of Pages 


\begin{tabular}{|c|c|c|c|c|c|c|c|c|c|}
\hline \multicolumn{10}{|c|}{ SI* (MODERN METRIC) CONVERSION FACTORS } \\
\hline \multicolumn{5}{|c|}{ APPROXIMATE CONVERSIONS TO SI UNITS } & \multicolumn{5}{|c|}{ APPROXIMATE CONVERSIONS FROM SI UNITS } \\
\hline Symbol & $\begin{array}{c}\text { When You } \\
\text { Know }\end{array}$ & $\begin{array}{c}\text { Multiply } \\
\text { By }\end{array}$ & To Find & Symbol & Symbol & $\begin{array}{c}\text { When You } \\
\text { Know }\end{array}$ & $\begin{array}{l}\text { Multiply } \\
\text { By }\end{array}$ & To Find & Symbol \\
\hline \multicolumn{5}{|c|}{ LENGTH } & \multicolumn{5}{|c|}{ LENGTH } \\
\hline in & inches & 25.4 & millimeters & $\mathrm{mm}$ & $\mathrm{mm}$ & millimeters & 0.039 & inches & in \\
\hline $\mathrm{ft}$ & feet & 0.305 & meters & $\mathrm{m}$ & $\mathrm{m}$ & meters & 3.28 & feet & $\mathrm{ft}$ \\
\hline yd & yards & 0.914 & meters & $\mathrm{m}$ & $\mathrm{m}$ & meters & 1.09 & yards & yd \\
\hline $\mathrm{mi}$ & miles & 1.61 & kilometers & $\mathrm{km}$ & $\mathrm{km}$ & kilometers & 0.621 & miles & $\mathrm{mi}$ \\
\hline \multicolumn{5}{|c|}{$\underline{\text { AREA }}$} & \multicolumn{5}{|c|}{$\underline{\text { AREA }}$} \\
\hline in $^{2}$ & square inches & 645.2 & $\begin{array}{l}\text { millimeters } \\
\text { squared }\end{array}$ & $\mathrm{mm}^{2}$ & $\mathrm{~mm}^{2}$ & $\begin{array}{l}\text { millimeters } \\
\text { squared }\end{array}$ & 0.0016 & square inches & in $^{2}$ \\
\hline $\mathrm{ft}^{2}$ & square feet & 0.093 & meters squared & $\mathrm{m}^{2}$ & $\mathrm{~m}^{2}$ & meters squared & 10.764 & square feet & $\mathrm{ft}^{2}$ \\
\hline $\mathrm{yd}^{2}$ & square yards & 0.836 & meters squared & $\mathrm{m}^{2}$ & $\mathrm{~m}^{2}$ & meters squared & 1.196 & square yards & $\mathrm{yd}^{2}$ \\
\hline $\mathrm{ac}$ & acres & 0.405 & hectares & ha & ha & hectares & 2.47 & acres & ac \\
\hline $\mathrm{mi}^{2}$ & square miles & 2.59 & $\begin{array}{l}\text { kilometers } \\
\text { squared }\end{array}$ & $\mathrm{km}^{2}$ & $\mathrm{~km}^{2}$ & $\begin{array}{l}\text { kilometers } \\
\text { squared }\end{array}$ & 0.386 & square miles & $\mathrm{mi}^{2}$ \\
\hline \multicolumn{5}{|c|}{ VOLUME } & \multicolumn{5}{|c|}{ VOLUME } \\
\hline $\mathrm{fl} \mathrm{oz}$ & fluid ounces & 29.57 & milliliters & $\mathrm{ml}$ & $\mathrm{ml}$ & milliliters & 0.034 & fluid ounces & $\mathrm{fl} \mathrm{oz}$ \\
\hline & gallons & 3.785 & liters & $\mathrm{L}$ & $\mathrm{L}$ & liters & 0.264 & gallons & gal \\
\hline $\mathrm{ft}^{3}$ & cubic feet & 0.028 & meters cubed & $\mathrm{m}^{3}$ & $\mathrm{~m}^{3}$ & meters cubed & 35.315 & cubic feet & $\mathrm{ft}^{3}$ \\
\hline & cubic yards & 0.765 & meters cubed & $\mathrm{m}^{3}$ & $\mathrm{~m}^{3}$ & meters cubed & 1.308 & cubic yards & $y d^{3}$ \\
\hline \multirow{2}{*}{\multicolumn{5}{|c|}{$\begin{array}{l}\text { NOTE: Volumes greater than } 1000 \mathrm{~L} \text { shall be shown in } \mathrm{m}^{3} \text {. } \\
\text { MASS }\end{array}$}} & \multirow{2}{*}{\multicolumn{5}{|c|}{$\underline{\text { MASS }}$}} \\
\hline & & & & & & & & & \\
\hline $\mathrm{oz}$ & ounces & 28.35 & grams & $\mathrm{g}$ & $\mathrm{g}$ & grams & 0.035 & ounces & $\mathrm{oz}$ \\
\hline $\mathrm{lb}$ & pounds & 0.454 & kilograms & $\mathrm{kg}$ & $\mathrm{kg}$ & kilograms & 2.205 & pounds & $\mathrm{lb}$ \\
\hline $\mathrm{T}$ & $\begin{array}{l}\text { short tons }(2000 \\
\text { lb) }\end{array}$ & 0.907 & megagrams & $\mathrm{Mg}$ & $\mathrm{Mg}$ & megagrams & 1.102 & short tons $(2000 \mathrm{lb})$ & $\mathrm{T}$ \\
\hline \multicolumn{5}{|c|}{ TEMPERATURE (exact) } & \multicolumn{5}{|c|}{ TEMPERATURE (exact) } \\
\hline${ }^{\circ} \mathrm{F}$ & Fahrenheit & $\begin{array}{l}(\mathrm{F}- \\
32) / 1.8\end{array}$ & Celsius & ${ }^{\circ} \mathrm{C}$ & ${ }^{\circ} \mathrm{C}$ & Celsius & $\begin{array}{l}1.8 \mathrm{C}+3 \\
2\end{array}$ & Fahrenheit & ${ }^{\circ} \mathrm{F}$ \\
\hline
\end{tabular}




\section{ACKNOWLEDGEMENTS}

The author would like to thank the members of ODOT's Technical Advisory Committee as well as the Research Section for their advice and assistance during the project and preparation of this report. The authors would also like to thank the undergraduate research assistants who contributed their time and effort to help complete this research and StarSeismic LLC that provided the Buckling Restrained Braces for the experimental portion of this project.

\section{DISCLAIMER}

This document is disseminated under the sponsorship of the Oregon Department of Transportation and the United States Department of Transportation in the interest of information exchange. The State of Oregon and the United States Government assume no liability of its contents or use thereof.

The contents of this report reflect the view of the authors who are solely responsible for the facts and accuracy of the material presented. The contents do not necessarily reflect the official views of the Oregon Department of Transportation or the United States Department of Transportation.

The State of Oregon and the United States Government do not endorse products of manufacturers. Trademarks or manufacturers' names appear herein only because they are considered essential to the object of this document.

This report does not constitute a standard, specification, or regulation. 


\section{TABLE OF CONTENTS}

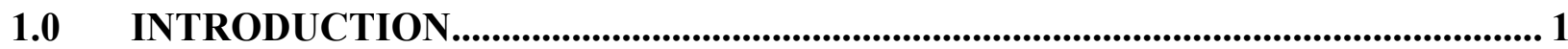

\subsection{INELASTIC STRUCTURAL RESPONSE TO SUBDUCTION ZONE}

EARTHQUAKES ................................................................................................................... 5

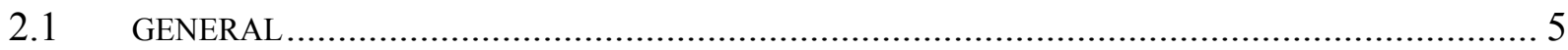

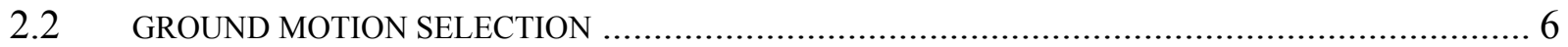

2.2.1 Representative Crustal Earthquake Set ………………………………………………………....6

2.2.2 Subduction Zone Earthquake Sets ……………………………………………………………....

2.2.3 Removing Outlying Records ……………………………………………………………...... 10

2.3 NUMBERICAL REPRESENTATION OF STRUCTURAL BEKHAVIOR …................................ 11

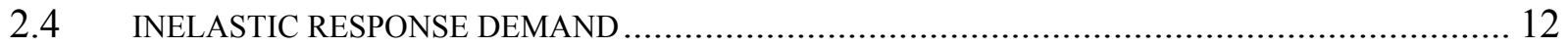

2.5 CUMULATIVE PLASTIC DISPLACEMENT DEMAND …………........................................ 14

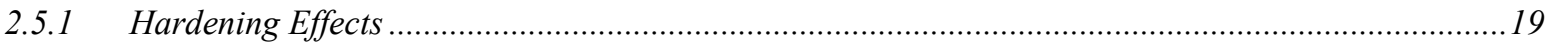

2.6 RESIDUAL DISPLACEMENT DEMAND ………………............................................... 19

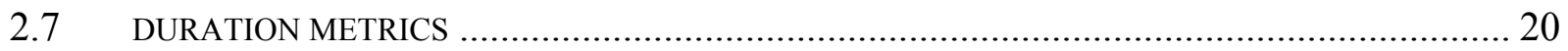

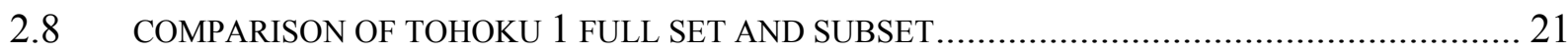

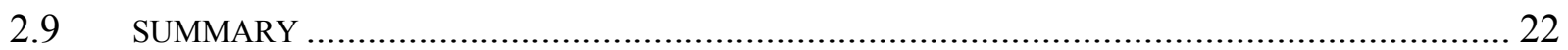

\subsection{CYCLIC LOADING PROTOCOL CONSIDERING SUBDUCTION MEGA}

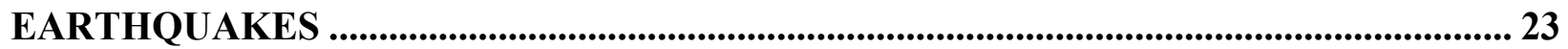

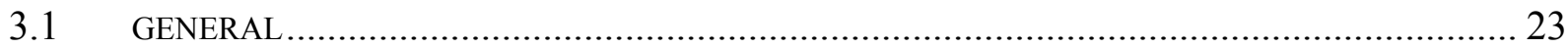

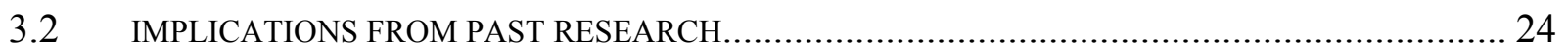

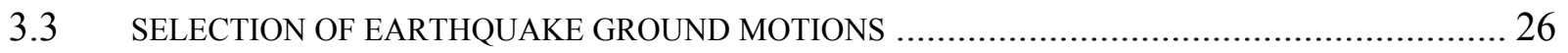

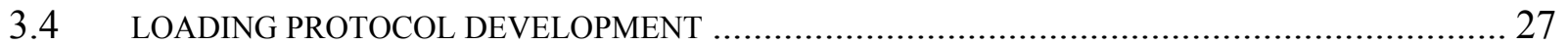

3.4.1 Hysteresis Model and Target Response .....................................................................................2

3.4.2 Cumulative Damage ……………………………………………………………………2.

3.4.3 Cycle Counting ……………………………………………………………………………...... 30

3.4.4 Results and Protocol Development .............................................................................................. 31

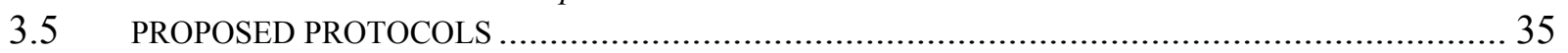

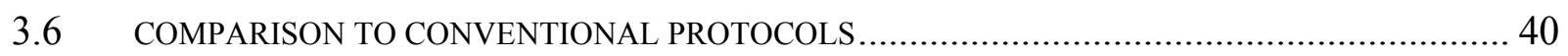

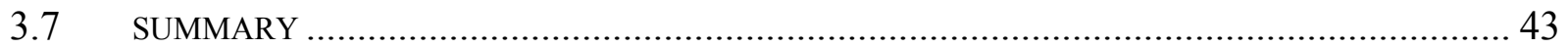

4.0 ASSESSMENT OF SEISMICALLY DEFICIENT RC BRIDGE COLUMNS ........ 45

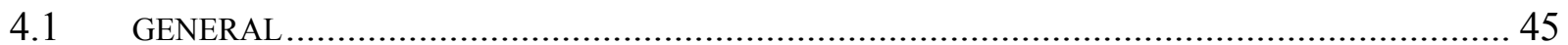

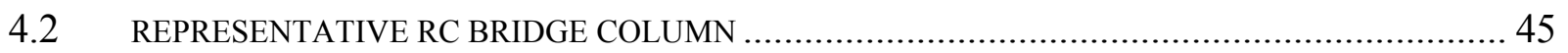

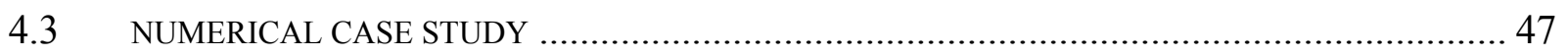

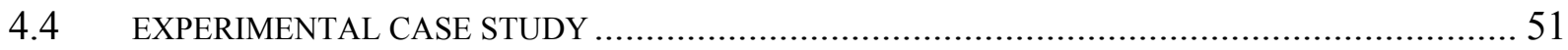

4.4.1 Test Program …………………………………………………………………………....

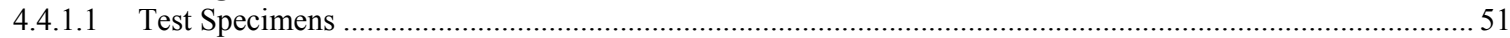

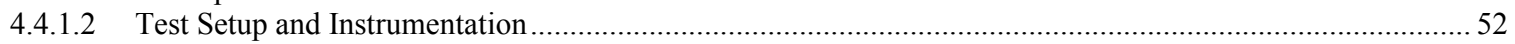

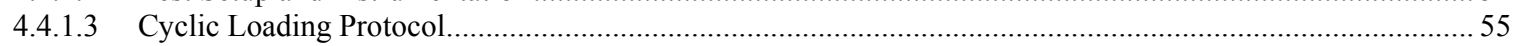

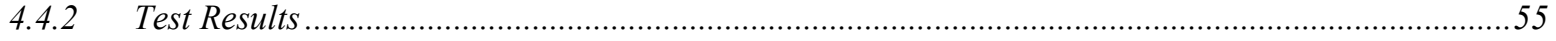

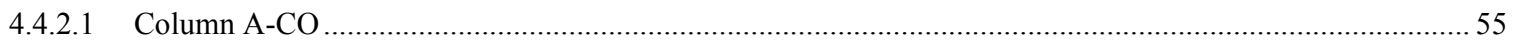

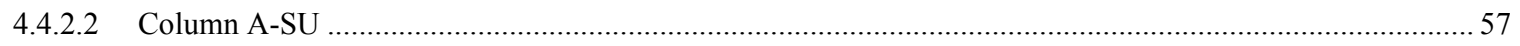

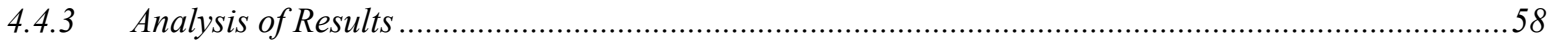

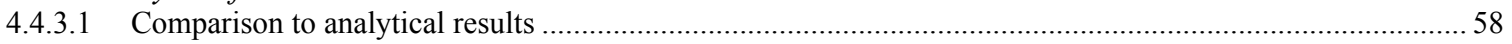

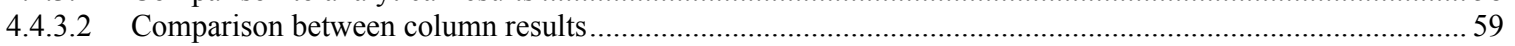

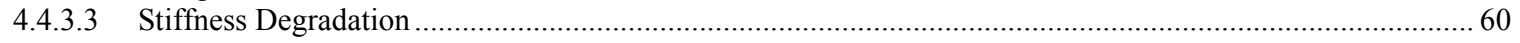




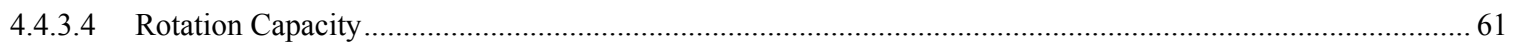

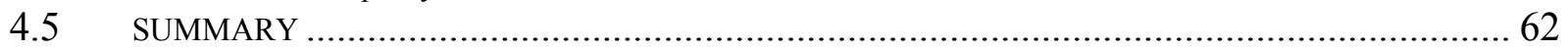

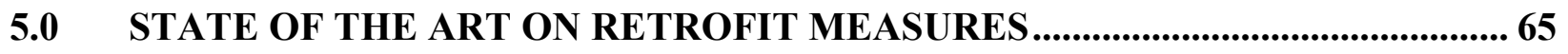

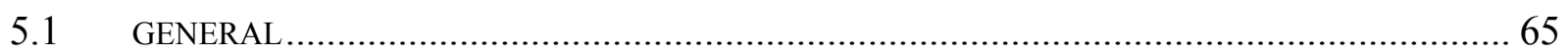

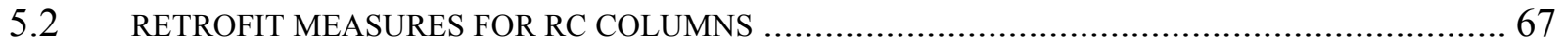

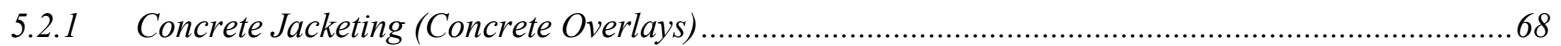

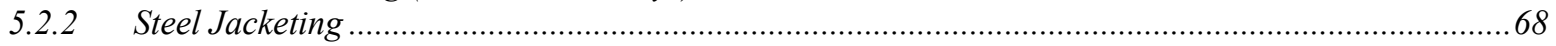

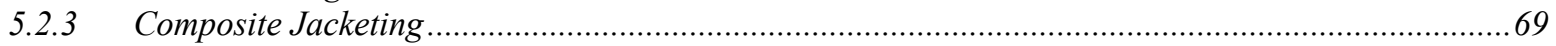

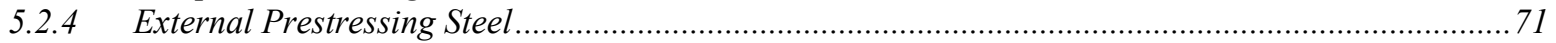

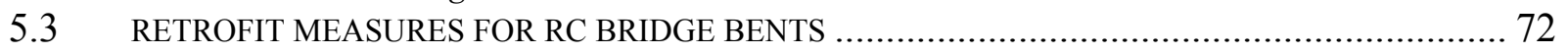

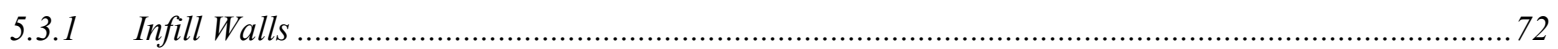

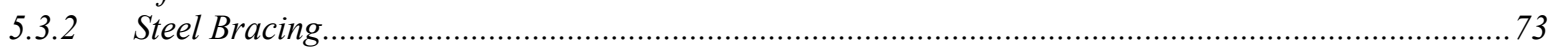

6.0 STRUCTURAL FUSES AS SEISMIC RETROFITTING MEASURE FOR MULTI-

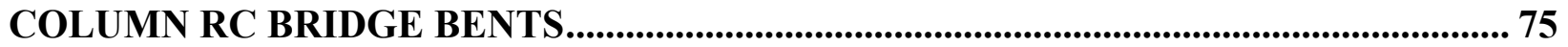

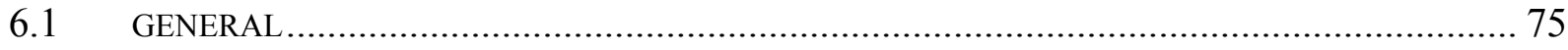

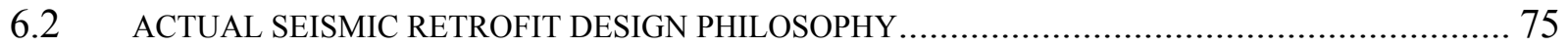

6.3 STRUCTURAL FUSE RETROFIT STRATEGY …........................................................ 76

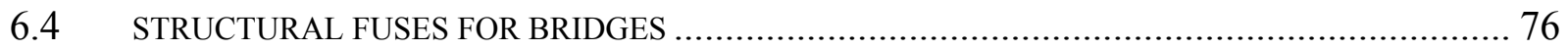

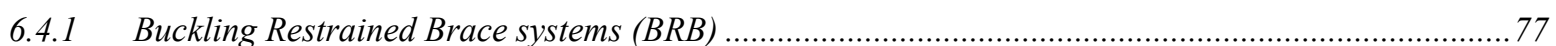

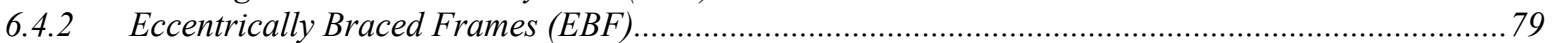

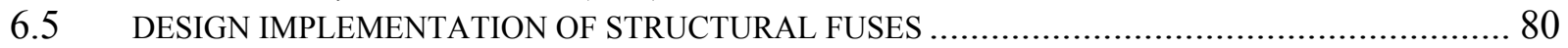

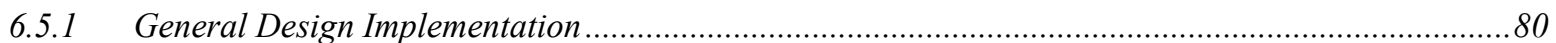

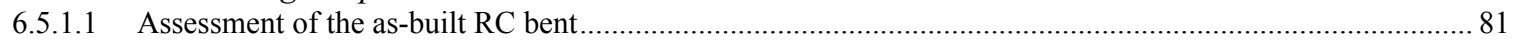

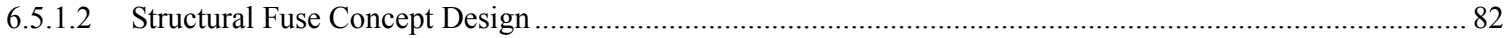

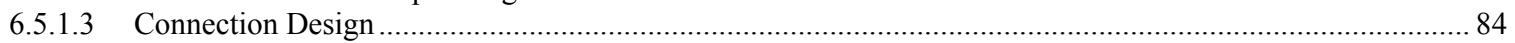

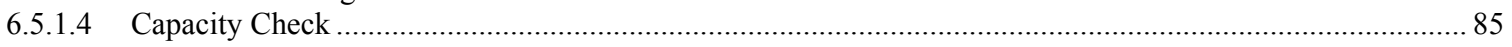

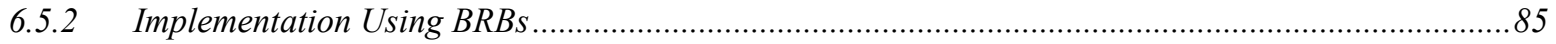

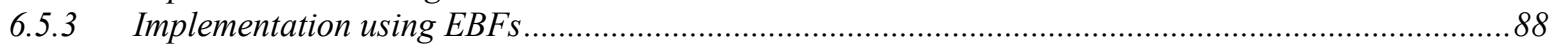

\subsection{ASSESSMENT OF RC BRIDGE BENTS UTILIZING BUCKLING RESTRAINED}

BRACES FOR SEISMIC RETROFIT ...........................................................................91

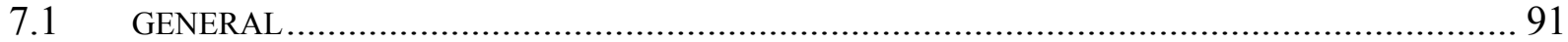

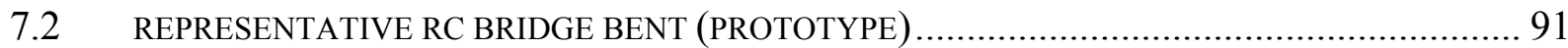

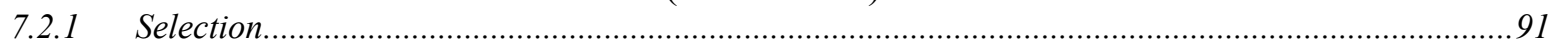

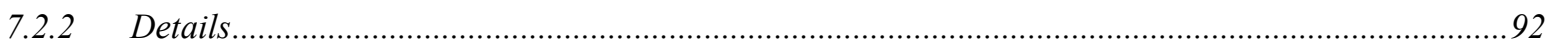

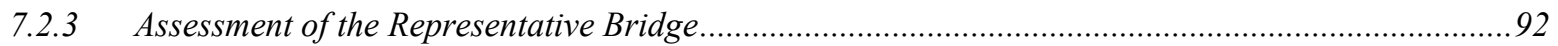

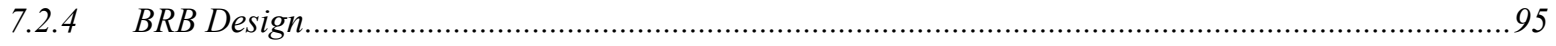

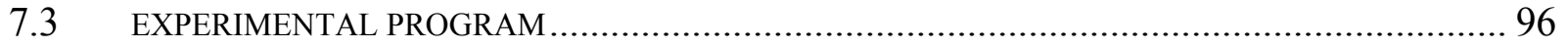

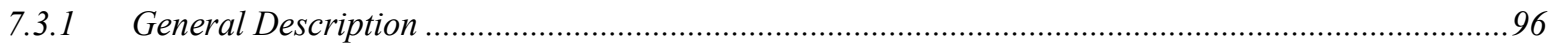

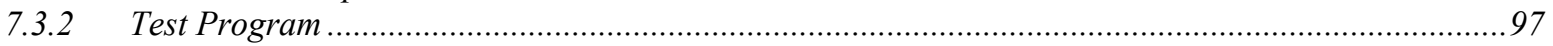

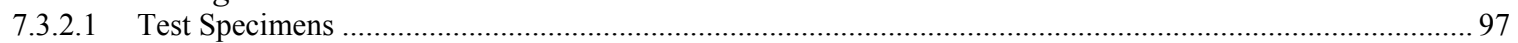

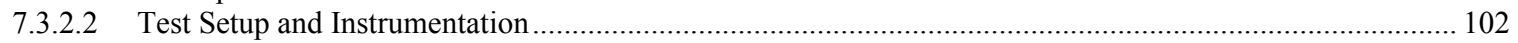

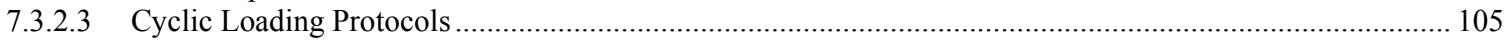

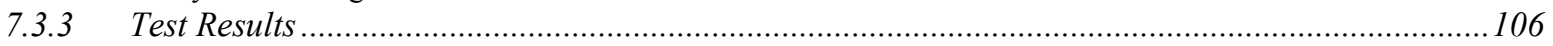

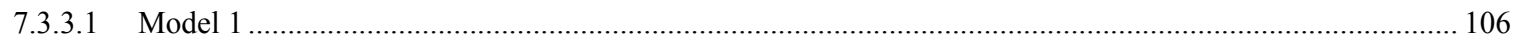

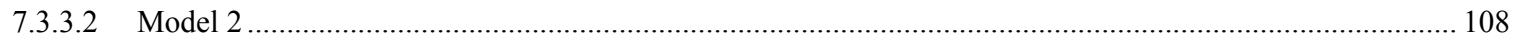

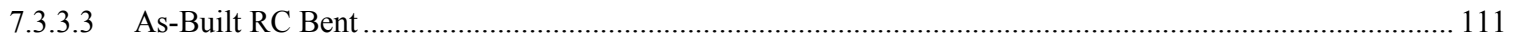

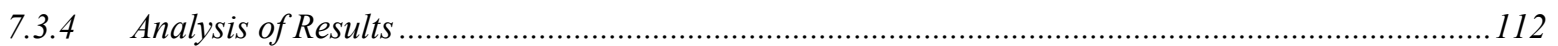

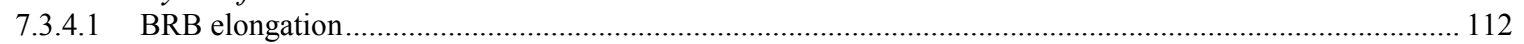

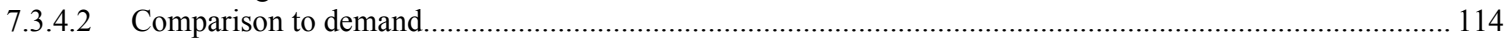

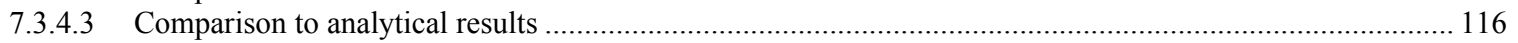

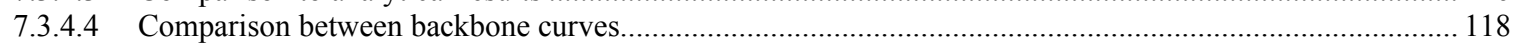




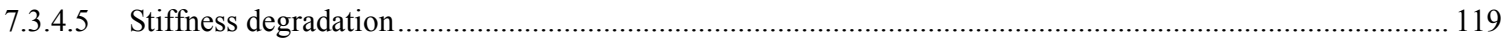

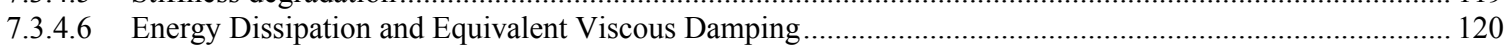

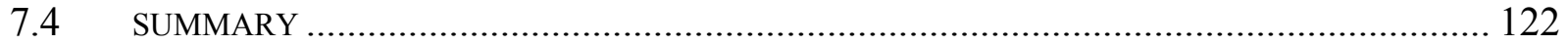

8.0 FRAGILITY CURVES .......................................................................................... 123

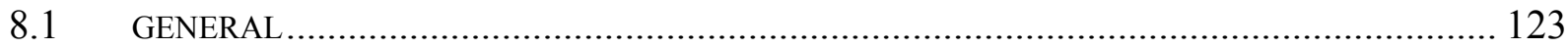

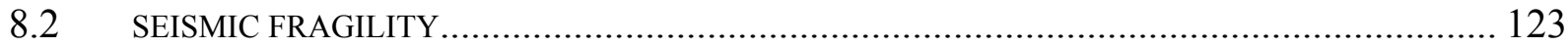

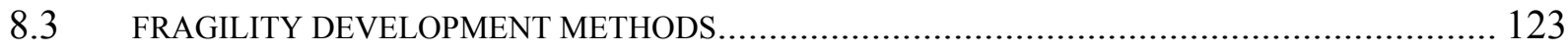

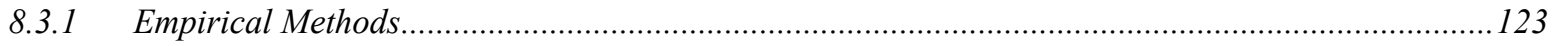

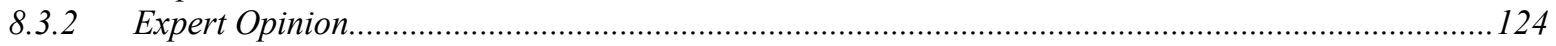

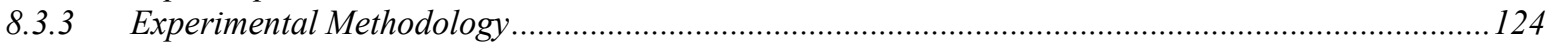

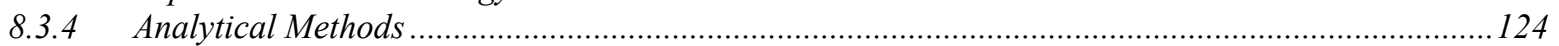

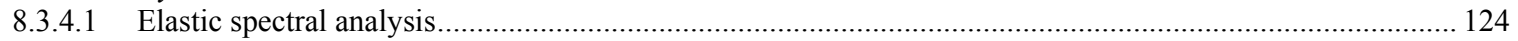

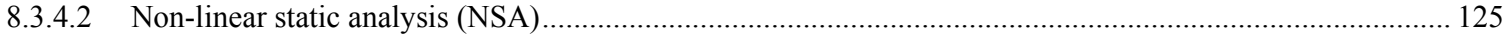

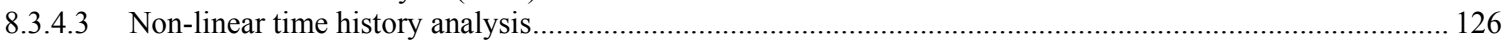

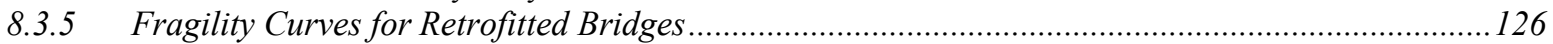

$8.4 \quad$ FRACILITY CURVES FOR REPRESENTATIVE BRIDGE BENT .................................... 127

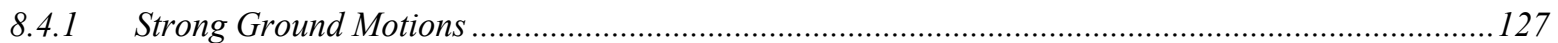

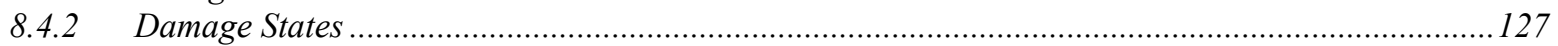

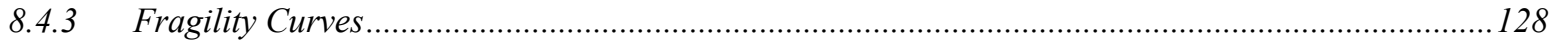

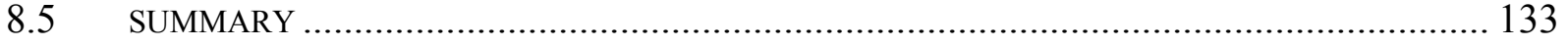

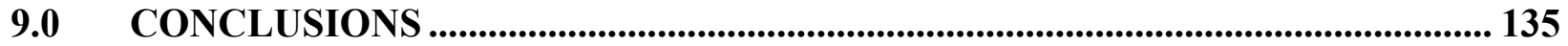

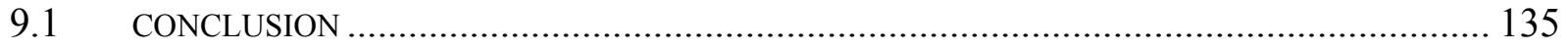

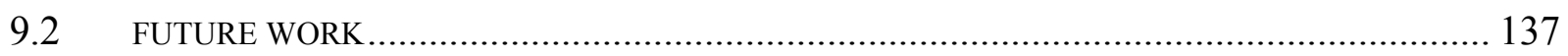

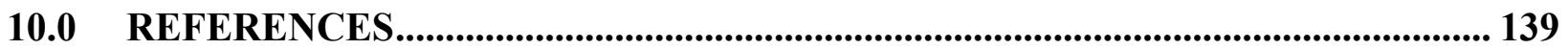

\begin{abstract}
APPENDICES
APPENDIX A: EARTHQUAKE CHARACTERISTICS

APPENDIX B: SCRIPTING PROCEDURE USED FOR NUMERICAL ANALYSIS

APPENDIX C: DEVELOPMENT OF THE NUMBERICAL MODELS

APPENDIX D: RESIDUAL DISPLACEMENT UPPER BOUND

APPENDIX E: ADDITIONAL RESULTS FROM CHAPTER 2

APPENDIX F: CYCLIC LOADING PROTOCOLS CONSIDERING ALL EXCURSIONS

APPENDIX G: MOMENT-CURVATURE ANALYSIS OF REPRESENTATIVE SQUARE

COLUMN

APPENDIX H: ADDITIONAL EXPERIMENTAL RESULTS FROM CHAPTER 4

APPENDIX I: DRAWING AND DESIGN OF CONNECTION

APPENDIX J: ADDITIONAL EXPERIMENTAL RESULTS FROM CHAPTER 7
\end{abstract}

\title{
LIST OF TABLES
}

Table 2.1: Mean Duration Metrics...............................................................................................................2

Table 3.1: Ground motion sets used for the development of cyclic loading protocols .........................................27

Table 3.2: Comparison of demand parameters for pre-peak and all excursions .................................................32

Table 3.3: Target values and proposed demands considering pre-peak excursions and all excursions .....................36 
Table 3.4: Proposed loading protocols

Table 3.5: Exponential coefficients to obtain cycle amplitudes ..........................................................................39

Table 3.6: Comparison of conventional protocols and proposed protocols ...........................................................41

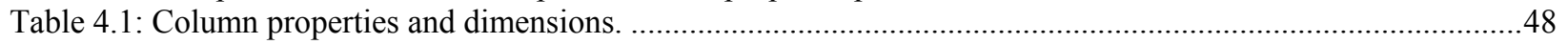

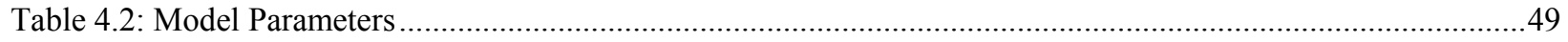

Table 4.3: Compressive strength of concrete cylinders for deficient square columns ........................................52

Table 7.1: Displacement capacity of the representative bridge bent .................................................................94

Table 7.2: Displacement demand in representative bridge bent ....................................................................95

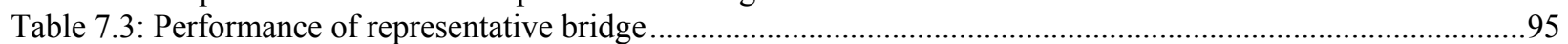

Table 7.4: Dimension of representative bridge and test specimen...................................................................99

Table 7.5: Compressive strength of concrete cylinders for deficient $\mathrm{RC}$ bent ....................................................99

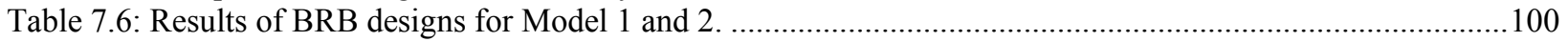

Table 8.1: Damage states for fragility curve development ....................................................................128

Table 8.2: Fragility curves values for representative RC bridge bent.............................................................. 132

Table 8.3: Fragility curves values for retrofitted RC bridge bent ................................................................. 132

\section{LIST OF FIGURES}

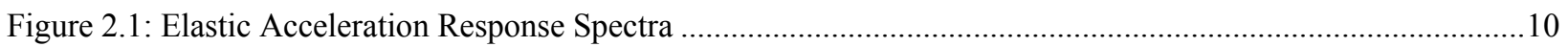

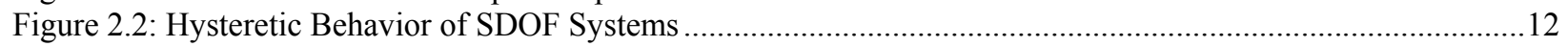

Figure 2.3: Mean Inelastic Acceleration Response Spectra......................................................................... 14

Figure 2.4: Mean NCPD Spectra: Bilinear and Degrading Models .................................................................. 17

Figure 2.5: Mean NCPD Spectra: Elastic Nonlinear Model ...........................................................................18

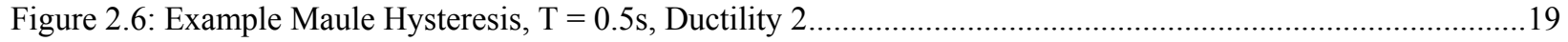

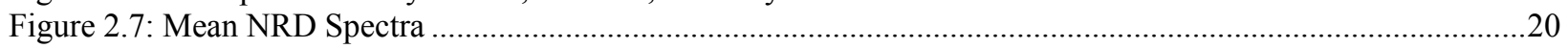

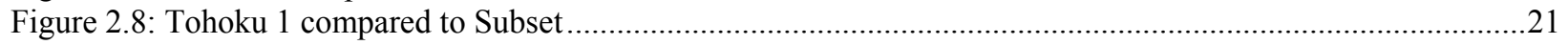

Figure 3.1: Conventional loading protocols used on RC column assessment. (a) New Zealand Protocol (Cheung et al. 1991), (b) Modified New Zealand Protocol (Priestley et al. 2002), (c) ACI374 unidirectional protocol (ACI

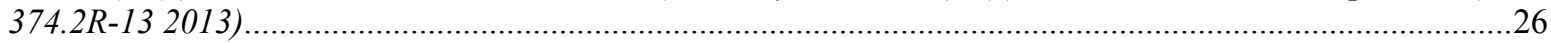

Figure 3.2: Hysteretic behavior of SDOF system. Adapted from Ibarra et al. (Ibarra et al. 2005a) ........................28

Figure 3.3: Illustration of the simplified rainflow counting procedure. (a) Inelastic response history. (b) Pre-Peak inelastic response history. (c) Ordered cycles including mean effect. (d) Final ordered cycles.........................31

Figure 3.4: Number of inelastic cycles for different component ductilities $(\mu)$ :

(a) Pre-Peak excursions; (b) All excursions...

Figure 3.5: Normalized Cumulative Plastic Deformation (NCPD) for different component ductilities ( $\mu$ ): (a) Pre-

Peak excursions; (b) All excursions.

Figure 3.6: Demand parameter values for different component ductilities.

(a) Number of Inelastic Cycles; (b) Normalized Cumulative Plastic Displacement (NCPD) ...........................35

Figure 3.7: Proposed loading protocols for component ductilities $(\mu)=2,4$ and 8.

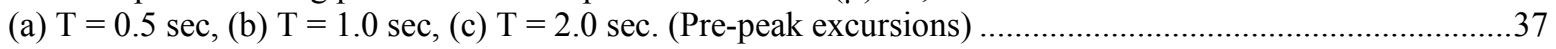

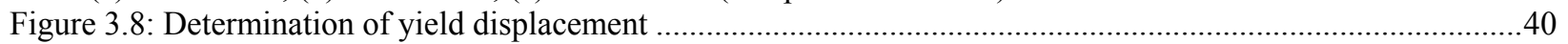

Figure 3.9: Illustrative comparison of protocols for bridge columns of ductility capacity 8 and period $0.5 \mathrm{sec} . . . . . . .41$

Figure 3.10: Comparison of cumulative ductility demands for component ductilities 2, 4 and 8 and component fundamental period of $0.5 \mathrm{sec}$.

Figure 3.11: Comparison of cumulative distribution functions for conventional protocols, proposed protocol, and statistical result for structures of period $0.5 \mathrm{sec}$ and different component ductilities. (a) $\mu=2$, (b) $\mu=4$, (c) $\mu=$

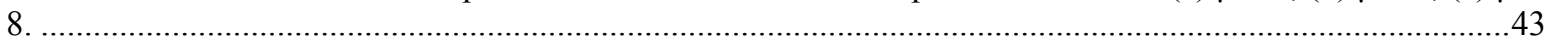

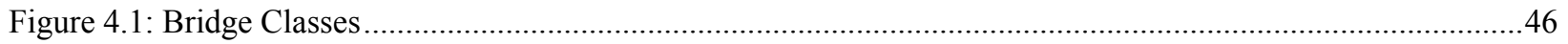

Figure 4.2: Illustration of seismic loading consideration over the years. ……........................................................46

Figure 4.3: Typical multi-span continuous stringer/girder RC bridge. (a) Picture. (b) Reinforcement details............47 Figure 4.4: Hysteretic behavior used for the numerical case study. Adapted from Ibarra et al. (Ibarra et al. 2005a) 48 Figure 4.5: Effect of loading protocol and model parameters on column response. (a) Standard Protocol. (b) Subduction Protocol. 
Figure 4.6: Geometry and reinforcement of RC Bridge column specimens .........................................................52

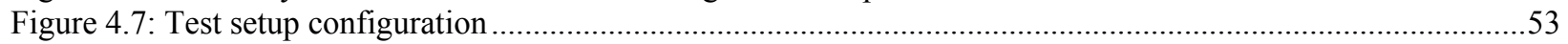

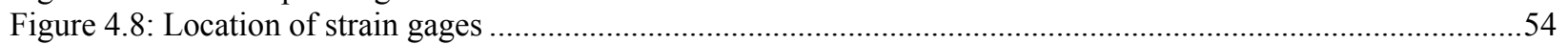

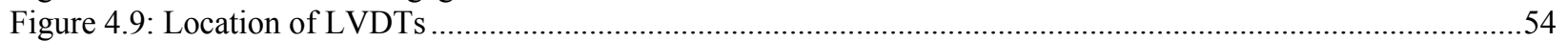

Figure 4.10: Damage progression in Column A-CO. (a) Horizontal cracks, (b) Crushing of concrete, (c) Bar buckling

Figure 4.11: Load vs. Displacement curve of column A-CO ............................................................................56

Figure 4.12: Damage progression in Column A-SU. (a) Horizontal cracks, (b) Crushing of concrete, (c) Bar buckling

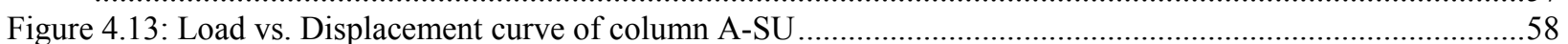

Figure 4.14: Experimental vs. analytical envelope curves for column A-CO .........................................................59

Figure 4.15: Experimental vs. analytical envelope curves for column A-SU ..........................................................59

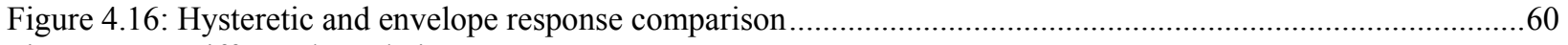

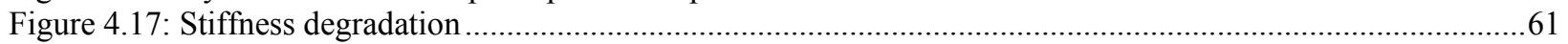

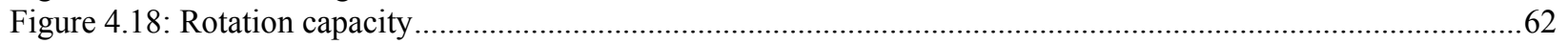

Figure 5.1: Poorly Confined Column in 1971 San Fernando Earthquake ……...................................................65

Figure 5.2: Collapsed Cypress street viaduct in Oakland during the 1989 Loma Prieta Earthquake (USGS and Wilshire 1989) ......................................................................................................................................6

Figure 5.3: Damage in RC columns on the Simi Valley Freeway during the 1994 Northridge Earthquake (Teng

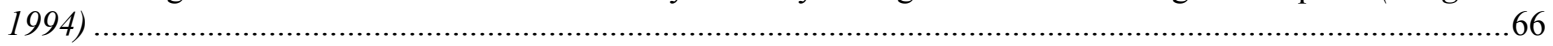

Figure 5.4: Damage in Nakasone viaduct during 2011 Japan earthquake (Kawashima et al. 2011) ........................66

Figure 5.5: Retrofit measure for bent caps. (a) Posttensioned rods. (b) Steel Jackets. (Wright et al. 2011)................67

Figure 5.6: Footing overlay on Hernando-Desoto Bridge (photo: R.A. Imbsen) ....................................................67

Figure 5.7: Concrete Jacketing. (a) Concrete overlay squematic (FHWA 2006), (b) Concrete jacketing of a column in Illinois (Poplar Street Complex) ..............................................................................................................68

Figure 5.8: Steel Jacketing for; (a) circular columns in bridge along Interstate 5, Seattle (photo: Lucia Bill), (b) rectangular column in bridge State Route 24 eastbound, San Francisco. Left: retrofitted column. Right: Asbuilt column (photo, Leonard G.).

Figure 5.9: Composite jacketing of a deficient square RC column. (a) Installation of CFRP, (b) Results (Mehary et

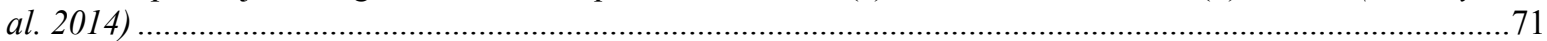

Figure 5.10: External prestressing steel retrofitting of columns in Illinois............................................................72

Figure 5.11: Effect of different retrofit measure for RC frames. (Sugano 1981) ...................................................73

Figure 5.12: Infill wall, (a) schematic, (b) Installation on West Lake Sammish Parkway (photo: L.M. Marsh).........73

Figure 6.1: Hysteretic behavior of a BRB. (Clark et al. 1999) .........................................................................77

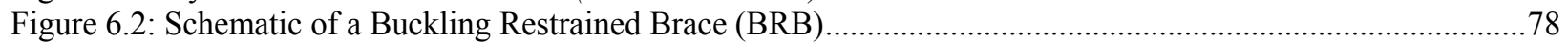

Figure 6.3: BRB retrofit configuration. (a) Structural fuse schematic. (b) Diagonal configuration. (c) Chevron

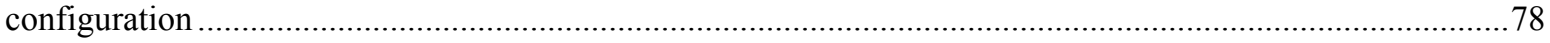

Figure 6.4: Different EBFs configurations. (AISC 2010) ……..........................................................................

Figure 6.5: Hysteretic behavior of EBF system. (Manheim 1982) ....................................................................79

Figure 6.6: Experimental study of using EBF system to retrofit RC structures. (Mazzolani 2009)............................80

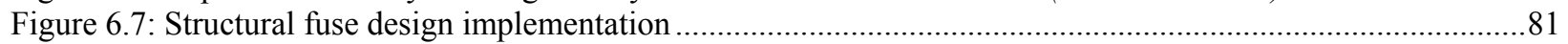

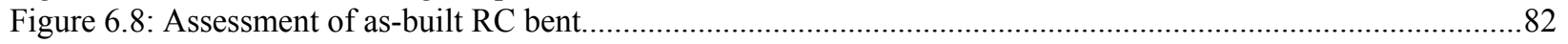

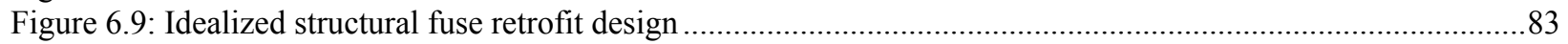

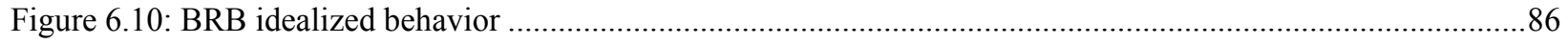

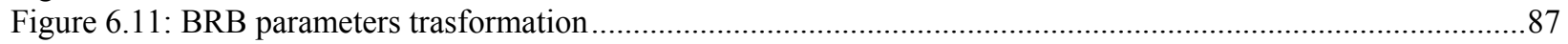

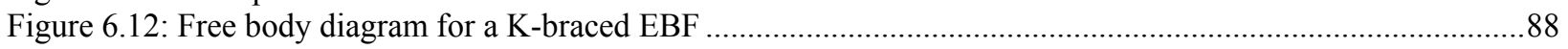

Figure 6.13: Effect of link length, e, in elastic lateral stiffness (Hjelmstad and Popov 1983) .................................88

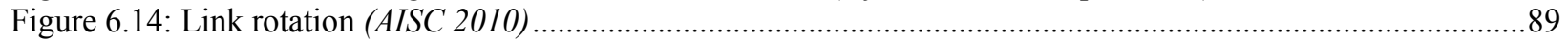

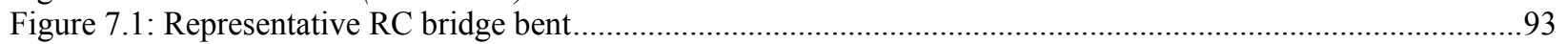

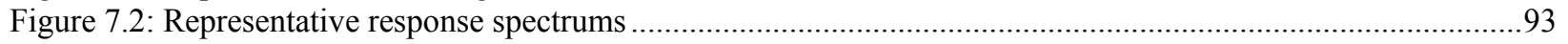

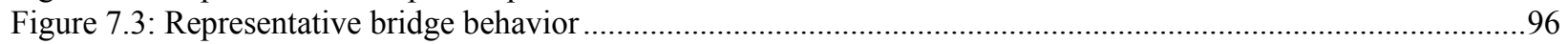

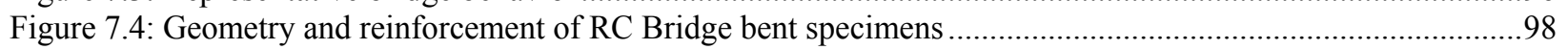

Figure 7.5: Expected load vs displacement behavior for Model 1 ...................................................................100

Figure 7.6: Expected load vs displacement behavior for Model 2 ...................................................................101

Figure 7.7: No Gusset Plate to Column Connection (a) Lower connection, (b) Top connection .............................102

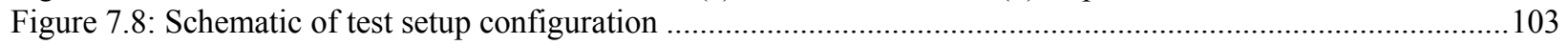


Figure 7.9: Actual test setup

Figure 7.11: Installation of strain gages. (a) Strain gages on longitudinal reinforcement and dowel, (b) Strain gages with protective coating.....

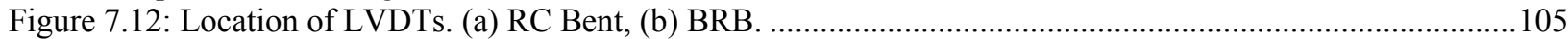

Figure 7.13: Cyclic loading protocol. (a) Retrofitted bent, (b) As-built RC bent...................................................106

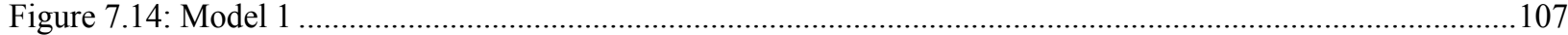

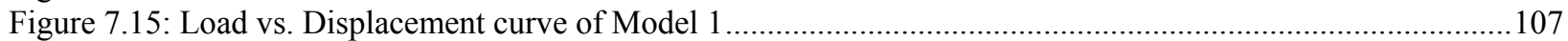

Figure 7.16: Damage in RC Bent Model 1. (a) Horizontal crack pattern, (b) Crack width .....................................108

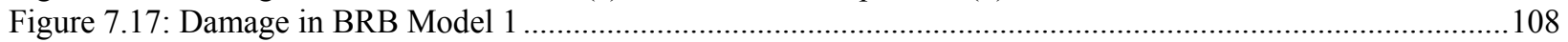

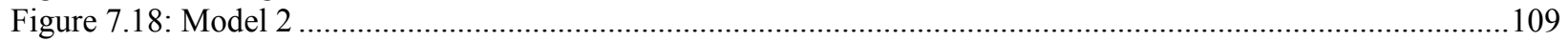

Figure 7.19: Load vs. Displacement curve of Model 2 ..............................................................................110

Figure 7.20: Damage progression in Model 2. (a) Horizontal cracks, (b) Spalling of concrete ..............................110

Figure 7.21: Damage in BRB Model 2 ................................................................................................111

Figure 7.22: Load vs. Displacement curve of As-built RC bent ......................................................................112

Figure 7.23: Damage on As-built RC bent. (a) Buckling of Steel, (b) Rupture of steel ........................................112

Figure 7.24: BRB elongation in Model 1. (a) Top and Bottom, (b) West and East, (c) Mean E-W and Bottom+Top

Figure 7.25: BRB elongation in Model 2. (a) Top and Bottom, (b) West and East, (c) Mean E-W and Bottom+Top

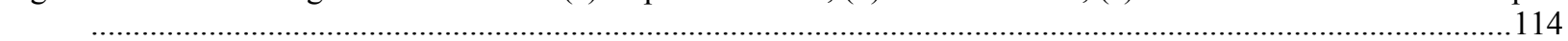

Figure 7.26: Comparison between experimental results and theoretical demand; (a) Model 1, (b) Model 2 ...........115

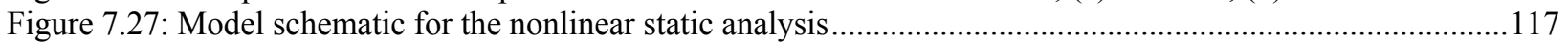

Figure 7.28: Experimental vs. analytical envelope curves for Model 1 ….........................................................117

Figure 7.29: Experimental vs. analytical envelope curves for Model 2 ................................................................117

Figure 7.30: Experimental vs. analytical envelope curves for the As-built bent ....................................................118

Figure 7.31: Backbone response comparison .............................................................................................119

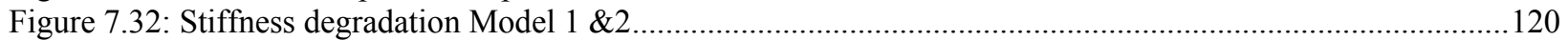

Figure 7.33: Illustration of energy dissipated through hysteretic behavior..............................................................120

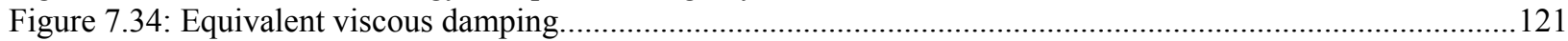

Figure 8.1: Capacity vs demand spectra showing uncertainty in structural behavior and ground motion response

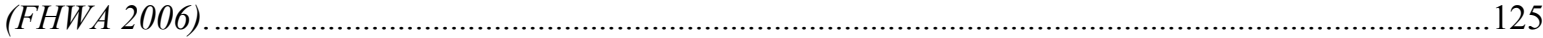

Figure 8.2: Response spectra for the 30 time histories (5\%damping) ................................................................127

Figure 8.3: Performance displacement of as-built RC bridge bent ....................................................................128

Figure 8.4: Performance displacement of retrofitted RC bridge bent ...............................................................129

Figure 8.5: Fragility curves for representative as-built RC bridge bent for damage state: (a) Slight, (b) Moderate, (c)

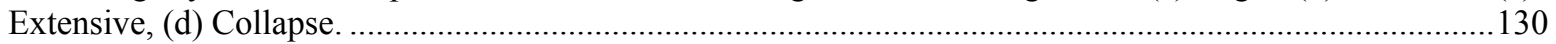

Figure 8.6: Fragility curves for representative as-built RC bridge bent...............................................................130

Figure 8.7: Fragility curves for retrofitted RC bridge bent (deterministic) for damage state: (a) Slight, (b) Moderate,

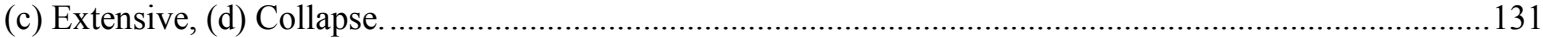

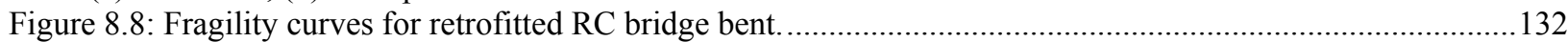

Figure 8.9: Comparison of median values of PGA for representative RC bridge bent..........................................132 


\subsection{INTRODUCTION}

Modern structural design philosophies rely on the inelastic response of structures for resisting earthquake ground motion. Seismological technology coupled with the frequent occurrence of crustal earthquakes has resulted in robust databases of crustal ground motions. The majority of earthquake studies have utilized crustal earthquakes for this reason. Subduction zone events, however, possess distinct differences in magnitude, duration, and frequency content compared to those of crustal earthquakes. Understanding how these differences affect structures is paramount to furthering the development of engineering practices. Recent subduction events and seismological evidence have brought to the forefront the importance of this to the area of the Pacific Northwest near the Cascadia subduction zone.

The Cascadia subduction zone is the over $1000 \mathrm{~km}$ long boundary between the Juan de Fuca and North American plates. Geological evidence has shown that 13 significant earthquake events have occurred in the past 3000 years (Goldfinger et al. 2008). The most notable of which, the M9.0 earthquake of 1700, produced a tsunami large enough to reach Japan (Atwater et al. 2005). Historical evidence combined with comparisons of the Cascadia fault to other subduction zones has led geologists to conclude that a megathrust earthquake in the Cascadia subduction zone is impending (Heaton and Kanamori 1984). This creates an even greater need for the more thorough understanding of the differences in structural response resulting from large subduction zone earthquakes, and consequently plan retrofit measures to mitigate the potential damage that these type of earthquakes poses in RC bridges.

Inelastic response of structures underlies most modern seismic design codes. Several studies involving analysis of inelastic structural demands of earthquakes have been conducted utilizing primarily crustal earthquakes (Krawinkler et al. 2003), (Ibarra et al. 2005a), (Ruiz-Garcia and Miranda 2003), (Ruiz-Garcia and Miranda 2007). Extending this research to large magnitude long duration subduction zone earthquakes has been limited by the availability of recorded ground motions as such earthquakes occur with less frequency than crustal earthquakes. Studies have been conducted with the few available ground motions (Stapleton et al. 2005), and several attenuation relationships have been developed (Atkinson and Boore 2003), (Gregor, et al. 2002) in order to study the effects of such earthquakes. Each approach has limitations affecting the broader applicability of the results. Using a small set of ground motions produces specific results that can not necessarily be extended to subduction zone earthquakes in general. Attenuation relationships often involve scaling procedures, which do not sufficiently account for regional variations and have been shown to produce biased results (Luco and Bazzurro 2007). Compiling a reasonable quantity of large motion subduction zone records was, therefore, desirable to produce results that could be more generalized.

This study differed from previous similar research through both the ground motions and the methodology used to study the response. Recent seismic events, specifically the 2011 Tohoku earthquake and the 2010 Maule earthquake, have increased the quantity and availability of long 
duration, large magnitude subduction zone ground motions to the extent that such a study was possible.

In Chapter 2 strong motion data from subduction zone earthquakes is analyzed through a constant ductility approach. This approach was utilized to determine inelastic demand differences among sets of earthquakes for different SDOF systems, and a metric was developed by which expected damage was quantified by taking into account the duration of the earthquake.

Capturing the mega subduction effect is of vital importance in the assessment of RC bridge columns located in the Pacific Northwest coast of the United States which lies near the Cascadia subduction zone and where a mega thrust earthquake of long duration forms a major component of the seismic risk. Results summarized in Chapter 3 aim to advance the seismic assessment of reinforced concrete bridge columns subjected to subduction zone earthquakes of long duration by developing a rational quasi-static loading protocol capable of simulating the demands imposed on bridge structures.

Chapter 4 presents numerical and experimental results of seismically deficient square reinforced concrete columns constructed before 1971 in the State of Oregon. These columns are commonly deficient in flexural ductility and shear strength as bridges were designed primarily for gravity loads without much consideration to lateral forces from seismic loading. The effect of long duration loading protocol on columns response was investigated.

Many bridges built before 1970 in the state of Oregon are seismically deficient. This vulnerability of pre-1970 bridges in the United States was especially evident in the 1971 San Fernando, 1989 Loma Prieta earthquake, and the 1994 Northridge earthquake. Further, the recent occurrence of subduction zone mega earthquakes in Chile and Japan has demonstrated how vulnerable RC bridges are when subjected to major seismic events. For that reason, improving the seismic capacity of deficient bridges have become a subject of paramount importance in seismic regions around the world. The main goal of any seismic retrofit measure is to minimize structural collapse, while meeting certain performance requirements. Nowadays, the number of available retrofit measures has increased markedly as a result of extensive analytical and experimental studies. In Chapter 5 conventional and emerging retrofit measures capable of improving the seismic resistance of deficient RC bridge substructures.

Seismic retrofit implementation in the State of Oregon has been minimal due to a lack of funding, and when retrofits have occurred, the scope was typically limited to retrofit by providing restrainers for keeping the superstructure from sliding from the supports. Such retrofit measures are effective for their intended purpose, but shift the displacement demands onto the supporting substructure. Designs for strengthening substructure elements for earthquake resistance are typically based on conditions in California, which typically has round bridge columns exposed to crustal seismic events. Oregon has many bridges with reinforced concrete bents and slender rectangular and round columns with inadequate reinforcement. Current seismic retrofitting manuals and guidelines for existing highway structures in Oregon are based on a performance-based design methodology, which uses a multiple-level approach to performance criteria with two seismic hazard levels. Therefore, engineers designing retrofit measures for improving the seismic resistance of standard existing bridge substructures need to ensure that the structure remains operational under a moderate earthquake and that life safety is preserved after a 
large earthquake. From the multiple retrofit alternatives presented in Chapter 5, in Chapter 6 is presented the option of using sacrificial elements also referred to as structural fuses as a retrofit measure for reinforced concrete bridges. Chapter 6 also presents the design implementation of these structural fuses for retrofitting RC bridge bents.

The actual implementation and response of RC bridge bents retrofitted by using buckling restrained braces are investigated in Chapter 7 via numerical and experimental studies. In that chapter a representative multi-column RC bridge bent is selected, assessed and retrofitted. Additionally, a half-scale RC bridge bent is tested using cyclic loading protocols that aim to represent displacement demands imposed in bridges by subduction zone mega earthquakes. The retrofit strategy presented in this study have the potential to improve the overall seismic behavior of RC bridges.

Analytical and experimental methods used to study typical vulnerable bents in Chapter 7 are used to quantify the seismic performance of the as-built and retrofitted bent with fragility curves. Chapter 8 presents the fragility curves for the as-built and retrofitted condition of the representative bridge bent. Thus, the effects of subduction zone earthquakes and retrofit measures can be better understood. 


\subsection{INELASTIC STRUCTURAL RESPONSE TO SUBDUCTION ZONE EARTHQUAKES}

\subsection{GENERAL}

Large magnitude megathrust subduction zone earthquakes are recognized to display distinct differences in acceleration magnitude, shaking duration and geographic region affected as compared to crustal earthquakes. Understanding the structural response differences to such earthquakes is paramount to furthering the development of engineering practices, particularly for affected regions such as the Pacific Northwest located near the Cascadia subduction zone. The Cascadia subduction zone is the over $1000 \mathrm{~km}$ long boundary between the Juan de Fuca and North American plates. Geological evidence has shown that 13 significant earthquakes have occurred in the past 3000 years (Goldfinger et al. 2008). The most notable of which, the M9.0 earthquake of 1700, produced a tsunami large enough to reach Japan (Atwater et al. 2005). Historical evidence combined with comparisons of the Cascadia fault to other subduction zones has led geologists to conclude that a megathrust earthquake in the Cascadia subduction zone is impending (Heaton and Kanamori 1984).

Due to the lack of available large magnitude subduction zone ground motion records, researchers wishing to analyze the structural demands of such earthquakes had in the past needed to resort to available records of smaller accelerations, conduct studies with the few ground motions, or utilized simulated records. Both simulated records and the use of attenuation relationships required ground motions to be scaled, which has the potential to produce biased results (Luco and Bazzurro 2007). Atkinson and Boore (Atkinson and Boore 2003) also stated that an earthquake of magnitude 8.0 or greater would result in a significant hazard increase compared to ground motions of lower magnitude, indicating the need for a study of the specific structural effects of large magnitude earthquake events. With the recent occurrences of the 2011 Tohoku, Japan (M9.0) and 2010 Maule, Chile (M8.8) earthquakes, the quantity of large magnitude subduction zone records increased to the extent that a study could be performed using recorded time history accelerations and thereby removing the bias caused by scaled or simulated records. Structural demand requirements produced by the Tohoku and Maule earthquakes do not necessarily directly extend to the Cascadia subduction zone, but by analyzing the demands, knowledge of the potential differences between Cascadia and crustal earthquakes can begin to be compiled.

This chapter outlines the method and results of numerical analyses utilizing a constant ductility approach that has the advantage of utilizing the strong motion records without the necessity of scaling. The responses of ductile single degree of freedom systems exhibiting bilinear or degrading hysteretic behavior were analyzed over a wide range of structural periods. The responses from subduction zone records were contrasted with results from representative crustal records and show that under the same maximum ductility, the cumulative plastic displacement demands can be two to three times that of crustal records for short to medium period structures. These findings could have impact on the damage expectations and on the evaluation criteria of bridge substructures following subduction zone earthquakes. 


\subsection{GROUND MOTION SELECTION}

The selection of ground motions was made with the aim of producing results of broad applicability. Ground motions were then compiled into sets for ease of comparison. These consisted of a representative crustal set and three subduction zone sets: two from the 2011 Tohoku earthquake and one from the 2010 Maule earthquake. Determining the different effects of near field and far field ground motions was beyond the scope of this study, so each set utilized only far-field records. This distinction further maintained the relevancy of this study to the Cascadia subduction zone, as the fault liles many miles from the Pacific Northwest coast.

\subsubsection{Representative Crustal Earthquake Set}

Crustal earthquake selection criteria and the uses of earthquake sets have varied among previous researchers. Krawinkler et al. (Krawinkler et al. 2003) studied the dependence of drift and ductility demands on ground motions and the relationships of the parameters to ground motion intensity measures. A set of 80 ordinary (not near-fault) ground motions from the PEER NGA database were compiled and divided into four bins according to magnitude and distance from the fault. The Large Magnitude-Short Distance (LMSR) bin caused the most critical structural response and was therefore utilized in subsequent studies which required a representative set of ordinary ground motion records. This included the work of Richards et al. (Richards et al. 2006) for the development of cyclic loading and testing protocol for eccentrically braced frames. LMSR earthquakes were defined as those with moment magnitude between 6.5 and 7.0 and distance from fault between $13 \mathrm{~km}$ and $30 \mathrm{~km}$ and included 20 records.

Medina and Krawinkler (Medina and Krawinkler 2003) determined the need to expand the number of records in the LMSR bin for statistical purposes to further quantify the dependence of seismic demands on ground motions. This expanded LMSR-N set was also used in further studies. One study analyzed the variance of collapse capacity of SDOF systems under earthquake excitations (Ibarra et al. 2011), while another demonstrated that deteriorating hysteretic models provide increased versatility and more realistic results for analyzing inelastic systems (Ibarra et al. 2005a). The LMSR-N record set consisted of 40 records and extended the distance requirement to include records up to $40 \mathrm{~km}$ from the fault. Both LSMR and LSMR-N records sets were limited to those earthquakes that originated from strike-slip, reverse-slip, or reverse oblique fault mechanisms, were recorded at Site Class D locations, and had high-pass frequency content less than $0.2 \mathrm{~Hz}$. No aftershocks were considered, but multiple records per event were included.

FEMA 695 (FEMA 695 2009) outlined an additional representative set of crustal earthquake ground motions from the PEER NGA database. The distinction between near-field and far-field records was again recognized. Selection criteria for the far-field record set were similar to that of the LSMR record set. The set contained 22 records that had moment magnitude greater than 6.5, were $10 \mathrm{~km}$ or greater from the fault, were recorded at locations of site class $\mathrm{C}$ or $\mathrm{D}$, and originated from strike-slip or reverse fault mechanisms. Additionally, only records from recording equipment located free-field or the ground floor or basement of a small building were considered. Arbitrary limits of $0.2 \mathrm{~g}$ minimum PGA and $15 \mathrm{~cm} / \mathrm{s}$ minimum PGV were imposed, and only the two records with the highest PGV per event were chosen. 
The FEMA far-field record set was similar to the previously used LSMR record set, but included a greater variety of events. As the set was also included as part of a procedure that is likely to be extensively used in structural system evaluations in the future, the FEMA far-field set was chosen as the representative crustal ground motion set in this study, and will be referred to as the Crustal set. The 22 ground motions along with their general characteristic and elastic response spectra can be found in Appendix A and Figure 2.1.

\subsubsection{Subduction Zone Earthquake Sets}

Prior to the recent events in Chile and Japan, there was a significant lack of large magnitude long duration subduction zone ground motion data. In order to study subduction zone affects, previous researchers utilized what records were available, modified records using attenuation relationships, or used simulated records.

Stapleton et al. (Stapleton et al. 2005) performed time history analyses of reinforced concrete columns by modifying the Llolleo, Chile 1985 (M 7.9) and Moquegua, Peru 2001 (M 8.4) earthquakes to fit a target acceleration spectrum for the Puget Sound region on the basis of an attenuation relationship developed by Atkinson and Boore (Atkinson and Boore 2003). Thompson (Thompson 2004) included the same generated earthquakes in a data set used for a corresponding numerical study. The data set totaled 10 ground motions, 6 of which were long duration records, and of those 4 were modified either through the attenuation relationship mentioned above or to fit an acceleration spectrum representative of the Seattle area.

Atkinson and Boore (Atkinson and Boore 2003) compiled an extensive subduction zone database including records from Mexico, Japan, and Central America of M 5-M 8.3. Though the quantity of earthquakes was significant, the majority were of relatively smaller magnitude. The database was used to develop attenuation relationships using a maximum likelihood regression method. A previous attenuation relationship used a stochastic finite-fault ground motion model and a less extensive record database to produce relationships specific to the Cascadia subduction zone (Gregor et al. 2002). This model was validated using the 1985 Miochoacan, Mexico (M 8.0) and 1985 Valpariso, Chile (M 8.0) earthquakes. Atkinson and Boore concluded that regression equations based on a global database did not sufficiently account for regional variability of ground motion amplitudes. Amplitude differences in Japan and Cascadia for events of the same characteristics were especially apparent. Results also indicated that the hazard produced by a $M>8$ earthquake would be significantly larger in terms of area experiencing damaging levels of ground motion than the hazard produced by a large in-slab event, suggesting the need for analysis of the effects of earthquakes of $M>8$.

A simulated earthquake for a Cascadia subduction zone event was developed by Yang (Yang 2009). This model utilized the empirical Green's function approach to produce a scenario Cascadia earthquake (M 9.2) using source parameters from the 2004 Sumatra-Andaman (M 9.2) earthquake. The 2003 Tokachi-Oki (M 8.1) earthquake was used as empirical Green's functions to simulate the ground motions. However, uncertainties in Cascadia site conditions compared to those in Japan produced large variations in the resulting equations, making the use of simulated ground motions undesirable when recorded motions are available. 
Japanese earthquakes were also used by Goda and Atkinson (Goda and Atkinson 2009) and Bai et al. (Bai et al. 2012). Goda and Atkinson (Goda and Atkinson 2009) studied the probabilistic distribution of the peak ductility demand of SDOF systems. Interface records were observed to vary significantly from inslab and crustal records. Large magnitude earthquakes were also shown to somewhat affect the distribution and suggested that even larger magnitude earthquakes than those used in the study $(\mathrm{M} \leq 8.3)$ could cause significant differences in inelastic demand. Bai et al. (Bai et al. 2012) considered larger magnitude earthquakes from the 2011 Tohoku event. A total of 6 ground motions, 3 from the Tohoku event and 3 simulated, were used to investigate the seismic demands of high-rise buildings models. The models were shown to not satisfy seismic demand under extreme ground motions.

Subduction zone ground motion sets used in this study were chosen with the intention of reducing the less desirable effects of ground motion selection in addition to focusing specifically on the effects of large magnitude subduction zone events compared to crustal events. The availability of ground motion data from the Tohoku and Maule events allowed for separate sets of records to be compiled. By not scaling or utilizing attenuation relationships, the earthquake characteristics were preserved. As each event had $\mathrm{M} \geq 8.8$, the records used did not appear in the database compiled by Atkinson and Boore (Atkinson and Boore 2003) or Goda and Atkinson (Goda and Atkinson 2009). This also allowed for the potential hazard resulting from a megathrust earthquake to be analyzed specifically. The two subduction events were similar in terms of magnitude, distance, and site classification as shown in Table 11, but records from the two events were analyzed separately in order to observe any potential differences caused by variations in regional site characteristics and soil profile that were not captured by the site classification procedure. The compiled record sets are also more extensive than those used by Stapleton el at. (Stapleton et al. 2005), Thompson (Thompson 2004), and Bai el al. (Bai et al. 2012) and can be considered to produce more statistically significant outcomes.

Due to the large amount of data available from the Tohoku earthquake on the Kyoshin Network (K-Net) database, the Tohoku records were further divided into two sets. The first contained records with PGA between $0.2 \mathrm{~g}$ and $0.9 \mathrm{~g}$, while the second contained records with PGA greater than $0.9 \mathrm{~g}$, referred to as Tohoku2 and Tohoku1 sets, respectively. The PGA range for the Tohoku 2 record set was chosen to produce a set with a similar PGA range and elastic acceleration spectral shape to the crustal and Maule sets. PGA ranged from $0.13 \mathrm{~g}$ to $0.93 \mathrm{~g}$ for the Maule set and $0.21 \mathrm{~g}$ to $0.82 \mathrm{~g}$ for the crustal set. Because the Tohoku2 set contained records with much higher PGA than the other sets, this set was kept separate in order to isolate the effects of records with larger PGA on the resulting structural demands. This difference in PGA range was also reflected in the shape of the elastic acceleration spectra shown in Figure 2.1.

The Tohoku2 record set contained components from 100 ground motions, while Tohoku1 contained 16 and Maule 19. The differing quantities of records directly reflect the availability of records from each earthquake. As the focus of this research was not to develop new criterion for selecting records, all available records available from the K-Net database in the determined PGA range were included. Additional records from the Tohoku earthquake were available from the Kiban-Kyoshin Network (Kik-Net) database, but as the two networks covered a similar geographical area, using only ground motions from the K-Net database was deemed appropriate. Two additional ground motions were also available from the Maule earthquake, but were not included as the PGA of each was orders of magnitude lower than the average PGA of the set. 
Earthquakes ground motions from the Maule earthquake were available via the Department of Civil Engineering at the University of Chile.

The disparity in the quantity of ground motions between the Tohoku2 and Maule sets was also addressed by selecting records from among the Tohoku2 set to form a subset of 19 records, referred to as the Tohoku2S set. No rigorous process was involved in the selection of Tohoku2S. The records were chosen simply to produce a subset with a similar PGA range and mean as the full set. Analyzing the structural response differences between the full set and the subset could give an indication as to the quantity of records necessary to produce confidence in the results, particularly when all of the ground motions in question are from one earthquake event. Elastic acceleration response spectra and record set characteristics are shown in Figure 2.1 and Appendix A-1.

In each of the record sets, two horizontal component records were used for each ground motion. Vertical components were not used. For the purposes of this study, component records were not rotated or combined, but treated as two separate ground motions. Imposed criteria for both the representative crustal and Tohoku sets were based on the component record that contained the higher PGA for each event, but each component were used in analysis regardless of PGA. This reflected the selection and analysis methods employed by the procedure set forth in FEMA 695 (FEMA 695 2009). Ground motions were also corrected prior to being used in this study. 

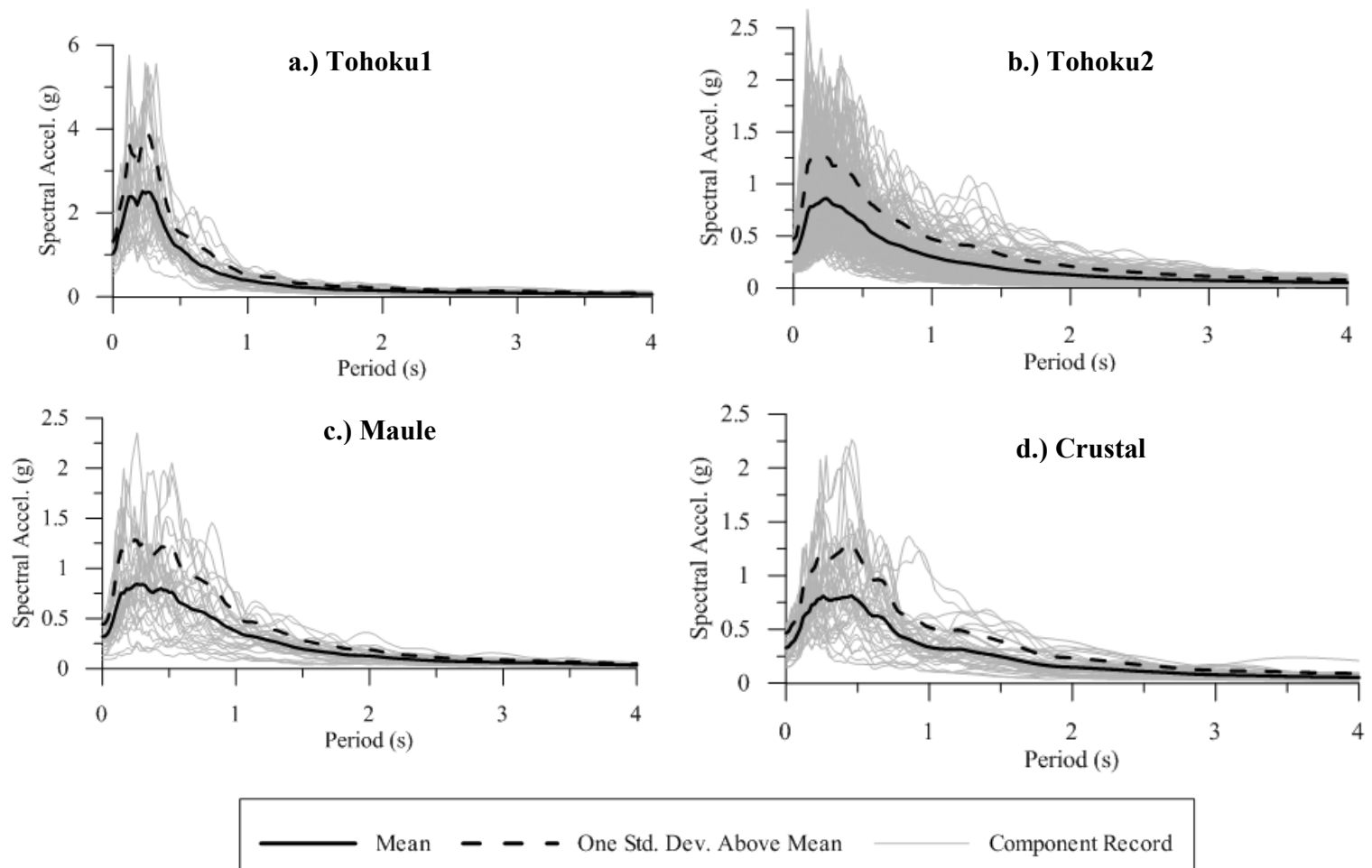

Figure 2.1: Elastic Acceleration Response Spectra

\subsubsection{Removing Outlying Records}

Evaluation of the elastic response spectra of each record set led to the observation that each set contained records that fell well above the mean at some point in the spectra. In order to avoid any potential skewing of the results, the outliers were removed. Common statistical methods of removing the outliers of a set do not apply when the set contains a series of spectra, rather than a series of single data points. A simple method was employed instead. Each component record that exceeded three standard deviations above the mean at more than one point of the elastic spectra was removed. Components that exceeded three standard deviations over the mean exactly once were not considered outliers. This was due to the fact that the spectra were calculated at small period steps of $0.02 \mathrm{~s}$. Records exceeding the limit only once at small period steps would most likely not exceed the limit if spectra were calculated at larger period steps, so exceeding the limit only once was not considered to be indicative of an outlying record. This effectively removed 7 components from the crustal set, 7 from the Maule set, 28 from the Tohoku2 set, 6 from the Tohoku2S set, and 5 from the Tohoku1 set. Outlying records were not included in Appendix A-1. Individual records for the Tohoku2 set were also not included, as the Tohoku2S set was considered to be representative of both sets. 


\subsection{NUMBERICAL REPRESENTATION OF STRUCTURAL BEKHAVIOR}

Representing structural behavior by single degree of freedom (SDOF) systems is a common analysis tool. Current code allows for the approximation of structures by SDOF systems, and utilizing SDOF systems maintains non-structure specific applicability. Analysis and computation time and complexity are also greatly reduced. In order to analyze the inelastic demands of various types of earthquakes, three SDOF systems were used.

Researchers have developed a variety of hysteretic models in order to produce representative results for a desired material behavior. Typically, a simple bilinear model was used and compared to more complex models. Such models included peak-oriented (Clough and Jonhnston 1966), pinching, and Bouc-Wen (Wen 1976) models. A CUREE study of wood frame structures, for example, utilized bilinear, peak-oriented, and pinching models (Krawinkler et al. 2002).

Rahnama and Krawinkler (Rahnama and Krawinkler 1993) developed hysteretic models that incorporated multilinear load-deformation response, strain hardening, stiffness degradation, strength degradation, and pinching characteristics. Peak oriented and pinching models were developed along with a deterioration factor. The deterioration factor was used to describe any combination of strength deterioration, degradation of unloading stiffness, and accelerated degradation of loading stiffness in the developed models.

More recently Ibarra et al. (Ibarra et al. 2005) developed a different strength and stiffness deteriorating hysteretic model. Basic strength, post capping strength, unloading stiffness and reloading stiffness cyclic deterioration modes were investigated. The proposed model expressed all cyclic deterioration modes by a single parameter and included a backbone curve with negative post-capping tangent stiffness, which was not included in the Rahnama and Krawinkler (Rahnama and Krawinkler 1993) model. This model maintained both simplicity and versatility. Ibarra et al. further concluded that using a model that included strength and stiffness degradation in an inelastic analysis process resulted in more accurate results.

This study utilized a bilinear model, an elastic non-linear model, and the peak oriented IbarraKrawinkler deterioration model (Ibarra et al. 2005). Each SDOF system was defined as a zero length element which utilized uniaxial material types available in OpenSees (OpenSees 2011). Both the bilinear and elastic non-linear models included 5\% post yield stiffness, and the IbarraKrawinkler model was calibrated based on the ductile moment frame model 3a used in FEMA 440A (FEMA 440A 2009).

FEMA 440A defined the model as points on a force deformation capacity boundary in terms of normalized force and drift. Drift was modified to normalized deformation in order to use the model as a zero length element, and the residual strength was adjusted to be $80 \%$ of the yield strength. The FEMA model represented structures of ductility level 8 , so this was scaled to produce additional capacity boundaries for structures of ductility levels 2 and 4 . When scaling, the stiffness of each leg of the capacity boundary was kept constant for ductility level 4, but this was not possible for ductility level 2 . The pre-capping leg was omitted and the post-capping 
stiffness was increased slightly in order to maintain the strength reducing and residual strength portions of the hysteresis. Hysteretic shapes for each model are shown in Figure 2.2. Appendix A-2 and A-3 show the procedure used for numerical analysis and parameter for each hysteretic model, respectively.

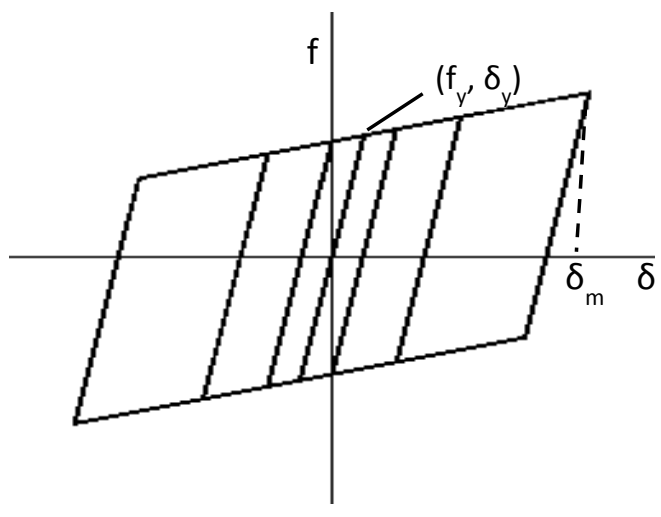

a.) Bilinear

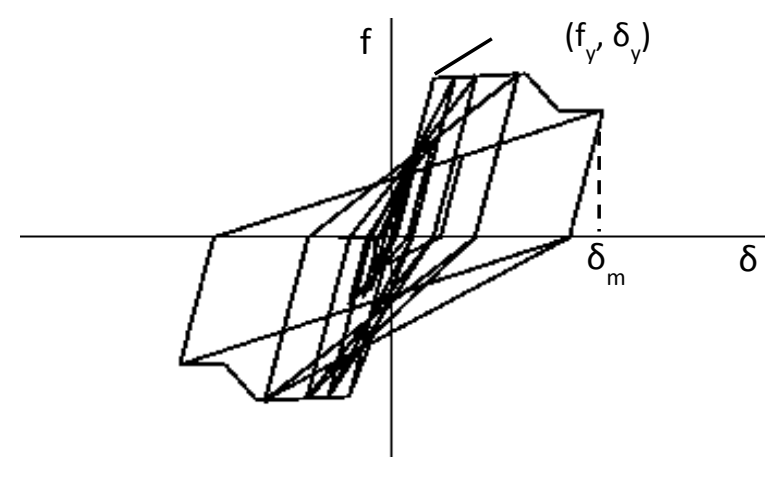

b.) Degrading

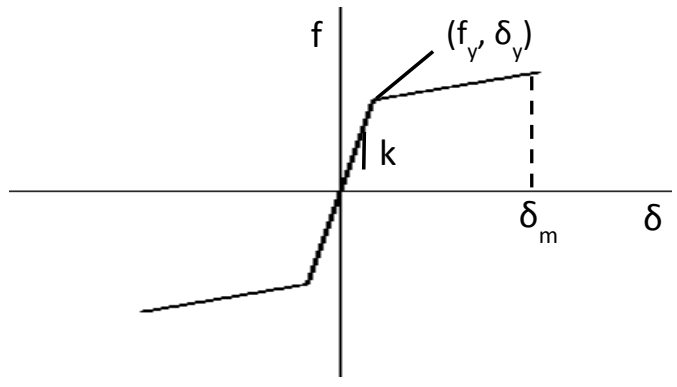

c.) Elastic Nonlinear

$f_{y}=$ yield force, $\delta_{y}=$ yield displacement, $\delta_{m}=$ max displacement, $k=$ stiffness

Figure 2.2: Hysteretic Behavior of SDOF Systems

The models were chosen and developed to be analyzed over a range of periods and ductility levels in order to represent a wide variety of structures. Steel component behavior was represented by the bilinear model, while the degrading model represented both reinforced concrete and ductile moment frame behaviors. The elastic nonlinear model was included to represent structures that display rocking behavior during earthquakes, as the benefits of utilizing rocking motion in structures are becoming more widely acknowledged and understood. To simplify the following explanation of results, the nonlinear behavior of elastic nonlinear model will be referred to as "plastic" behavior.

\subsection{INELASTIC RESPONSE DEMAND}

Researchers have generally used one of two methods to analyze structural systems for seismic demand: a constant strength approach or a constant ductility approach. In each approach, structures are commonly approximated as inelastic SDOF systems. The constant strength 
approach assigns known strengths to the system to solve for the peak ductility demand, while the constant ductility approach finds the yield strength that produces a desired peak ductility value. Each approach produces the desired seismic demand of a structure over a range of natural periods and as a result of multiple earthquake ground motions.

The constant strength approach determines inelastic displacement demands probabilistically. Methods have been developed to analyze the ductility demand of existing structures (RuizGarcia and Miranda 2003), as well as structures which display Bouc-Wen hysteretic behavior (Goda et al. 2009). This has also been used as a means of estimating maximum inelastic displacement demand as a part of performance-based design (Ruiz-Garcia and Miranda 2007). A constant strength approach was further used as mechanism for determining the sensitivity of inelastic SDOF systems to the orientation of input ground motions (Goda 2012). In each study, ground motions were scaled to produce a constant seismic excitation relative to the yield strength of the structure. The advantage of this approach is the applicability to existing structures. Luco and Bazurro (Luco and Bazurro 2007) have demonstrated that scaling of ground motion records can produce biased results. As the focus of this study was to determine the effects of different types of ground motions, analysis procedures that called for scaling of ground motions were avoided.

The constant ductility analysis procedure used in this study was closely related to the inelastic response spectra procedure used by Murukami and Penzien (Murukami and Penzien 1975). Time history analysis of each of SDOF systems over a range of natural periods up to $4 \mathrm{~s}$ at $0.02 \mathrm{~s}$ increments was conducted with each of the earthquake ground motions using OpenSees (OpenSees 2011). Due to numerical computation issues at very small periods, analysis of the degrading model began at $0.1 \mathrm{~s}$. At each period and for each earthquake record, the yield strength of the structure that produced the desired ductility value was found through an iterative process, in effect having an individual ductility design for each of the earthquake records. Krawinkler and Nassar (Krawinkler and Nassar 1990) referred to this type of spectra as strength demand spectra. Miranda (Miranda 1993) conducted a similar evaluation of site-dependent inelastic seismic demand spectra. Rahnama and Krawinkler (Rahnama and Krawinkler 1993) expanded the approach to determine the effects of soft soils and hysteresis models on strength demand. The ductility, $\mu$, was defined as

$$
\mu=\frac{\delta_{m}}{\delta_{y}}
$$

Where, $\delta_{\mathrm{m}}$ is the max displacement and $\delta_{\mathrm{y}}$ is the yield displacement. Inelastic responses were calculated at ductility values of 2,4 , and 8 as this was considered to represent a wide range of ductile structural responses. Throughout the process, mass of the system remained constant, while the stiffness, $\mathrm{k}$, was calculated based on the desired period. Yield force was represented by $f_{\mathrm{y}}$.

Resulting inelastic acceleration spectra were analyzed by comparing the mean of each record set for each model. Spectra were found to differ very little between models, so only the results of the bilinear model are shown in Figure 2.3. An example of how the models compare is also shown for the Maule set at ductility 2. For this and the presentation of the majority of the results, the Tohoku2S set was omitted, as providing results for both the Tohoku2 and Tohoku2S sets was 
redundant. An isolated comparison of the two sets follows. The mean of the Tohoku1 response was consistently higher than the other record sets, which was a direct reflection of the higher acceleration content of that set. The Tohoku2, Maule, and Crustal sets, however, resulted in very similar values despite the differences in fault type and magnitude of the earthquake events.
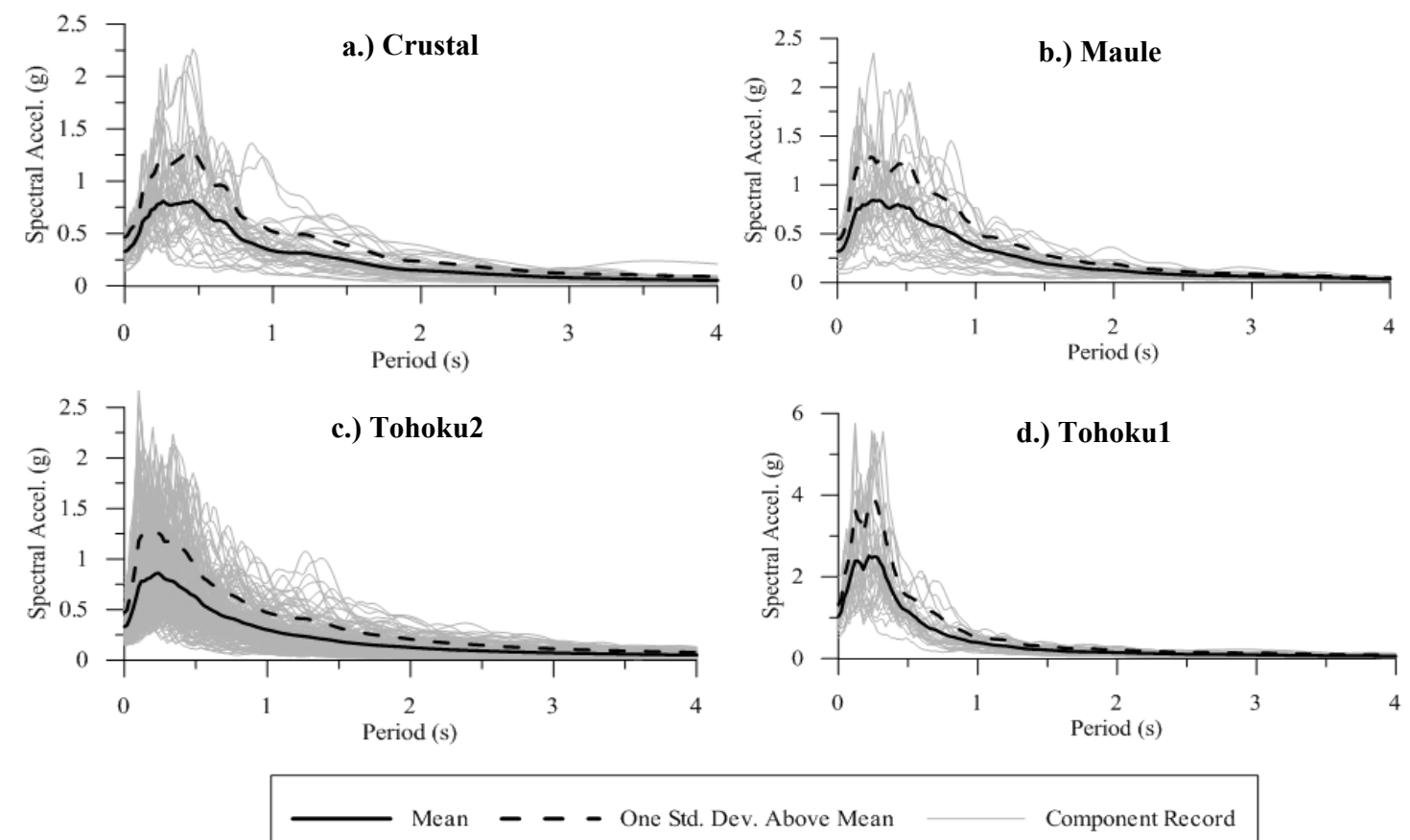

Figure 2.3: Mean Inelastic Acceleration Response Spectra

\subsection{CUMULATIVE PLASTIC DISPLACEMENT DEMAND}

Force based seismic design is evolving towards displacement based design from the research realm to practice, whereby a number of states including Oregon have adopted Guide Specifications (AASHTO 2009) to more appropriately capture the structural behavior. Hence, a displacement based metric was needed to compare the seismic demand between record sets that would take into account the longer duration of subduction zone records and allow for comparison between ground motions of various magnitudes and spectral acceleration contents. This was achieved by calculating the cumulative plastic displacement demand over the duration of the response.

To calculate cumulative plastic displacement, elastic displacement was first removed from the total displacement leaving only the plastic displacement, up, at each time increment of the analysis, "i". Adding the incremental differences of plastic deformation produced the cumulative plastic displacement, $U_{p}$, for each earthquake record at each structural period as 


$$
U_{p}=\sum_{i=1}^{n}\left\|\left(\delta_{p, i+1}-\delta_{p, i}\right)\right\|
$$

Normalizing this value by the yield displacement at each period produced a unitless metric by which the seismic demand of earthquakes of different types and magnitudes could be compared among different systems. Since the structures were designed to a set ductility value, i.e. to a specific inelastic displacement demand, the differences in the normalized cumulative plastic displacement (NCPD) indicate the amount of plastic demand imposed. The results can be presented for each earthquake by plotting the data over the period range at each ductility value. These plots are referred to as normalized cumulative plastic displacement spectra.

Resulting plastic displacement demand varied among models as illustrated in Figure 2.4 and Figure 2.5. Different trends were also apparent for higher and lower periods, particularly for the bilinear and degrading models. For the bilinear model, at periods of less than $0.5 \mathrm{~s}$, higher demands were produced by the Tohoku1 and Maule sets than the Tohoku2 set when compared to the Crustal set. At the peak of the Crustal demand, the Tohoku1 and Maule sets resulted in an average of $20 \%$ and $40 \%$ more demand, respectively. This means that for lower period bilinear structures designed to the same ductility, the Tokoku2 and Maule earthquakes accumulated noticeably more plastic deformation.

At periods higher than approximately $1.0 \mathrm{~s}$, both Tohoku sets produced lower normalized cumulative displacement demand than the Crustal set. This difference increased as the period increased and was more pronounced at higher ductility values. For the analysis period of $4.0 \mathrm{~s}$, the mean NCPD produced by the Tohoku1 set was $41 \%, 49 \%$, and $58 \%$ less than that of the Crustal set for ductility values 2,4 , and 8 , respectively. Despite the differences in spectral acceleration content, the mean NCPD demand of the Tohoku sets remained within approximately $18 \%$ of each other for all ductility values. This means that despite the longer duration of the records, the accumulated plastic deformation was significantly less for the Tohoku sets than the Crustal set for high period structures. Conversely, the Maule set produced more demand than the Crustal set at higher periods. Again, an increase in demand was seen as the ductility increased with the Maule set producing 26\%, 55\%, and 62\% more demand than the Crustal set at increasing ductility levels. The contrasting behavior of the Maule and Tohoku sets was notable, because the results were not what would be expected based on commonly used intensity measure such as acceleration spectra and magnitude.

In contrast to the bilinear model, the elastic nonlinear model did not result in significant trend differences among higher and lower period structures. All subduction sets produced consistently higher demand than the Crustal set. At both the peak of the Crustal demand and at the 4.0s analysis period, subduction sets averaged approximately $60 \%, 120 \%$, and $170 \%$ higher demand at increasing ductility levels. All structures with this type of behavior over the range of periods analyzed accumulated significantly more damage due to subduction earthquakes than crustal earthquakes. The degree to which damage accumulated was also orders of magnitude higher for the elastic nonlinear model than for the bilinear model for structures designed to the same levels of ductility ranging from approximately 3 to 10 times more demand at the peak of the mean Crustal response. 
The NCPD spectra for the degrading model displayed a different spectral shape than the previous models and the produced plastic displacement demand was orders of magnitude higher than that of the elastic nonlinear model at lower periods. The trend differences between higher and lower period structures that were seen in the bilinear model were seen in the degrading model, though the trends were more apparent as ductility increased and the transition between lower and higher period effects was not as clear. In this case, all subduction zone sets produced more NCPD demand than the Crustal set for lower period structures, whereas the Tohoku2 set of the bilinear model tracked more closely to the Crustal set. At the peak of the NCPD response, all subduction sets produced over 200\% more demand than the Crustal set. The largest differences in demand produced by the degrading and elastic nonlinear models occurred at ductility 2 where the demand of the degrading model was approximately 3 to 6 times greater than the elastic nonlinear model and fell to approximately 1.3 to 2.0 times greater at ductility 8 .

The transition to higher period effects occurred at a lower period as ductility increased, but generalizations could be made about the behavior of structures higher than approximately $2 \mathrm{~s}$. As with the bilinear model, the demand produced by the Tohoku sets of the degrading model was significantly less than that of the Maule set, around $40 \%$ to $80 \%$. All subduction sets produced higher demand than the Crustal set, but particularly for the higher period structures designed to higher levels of ductility the difference in demand produced by the Tohoku and Crustal sets was negligible. Generally, the demand produced by the degrading model at higher periods was of similar magnitude to that produced by the elastic nonlinear model.

The elastic nonlinear and degrading models further confirmed that lower period structures are likely to be susceptible to increased demands due to subduction zone earthquakes. The degree of damage seemed to be dependent on the behavior of the structure and was more apparent when strength and stiffness degradation occurred. Among higher period structured, the Maule set produced at least as much demand as the Tohoku sets, in most cases more. Based on the magnitude and acceleration spectra of each set, the most demand would be expected to result from the Tohoku1 set. The cumulative plastic displacement metric, therefore, captures structural damage that was not possible with acceleration spectra.

Hysteretic behavior of each model can be used to explain the magnitude of demand differences displayed above. As an example, the normalized force displacement curves for one Maule earthquake designed to a $0.5 \mathrm{~s}$ period and ductility of 2 are shown in Figure 2.6. The elastic behavior of the bilinear model can be clearly seen as each segment that is parallel to the initial stiffness. Cumulative displacement was higher for the elastic nonlinear model, because with each excursion that caused the structure to change from plastic displacement in one direction to the other direction the elastic nonlinear model had to backtrack through a certain amount of "plastic" deformation before reaching the elastic region again. The bilinear model, in contrast, was able to move into an elastic region as soon as the displacement became small enough.

The even larger plastic displacement of the degrading model was an effect of the reloading stiffness degradation. In each cycle, the structure behaved plastically between the yield point and the beginning of unloading, behaved elastically during unloading, but moved back into the plastic region during reloading due to the reduction in reloading stiffness that is characteristic of pinching behavior. This additional plastic portion of the hysteresis can explain the higher cumulative plastic displacement demand of the degrading model. 

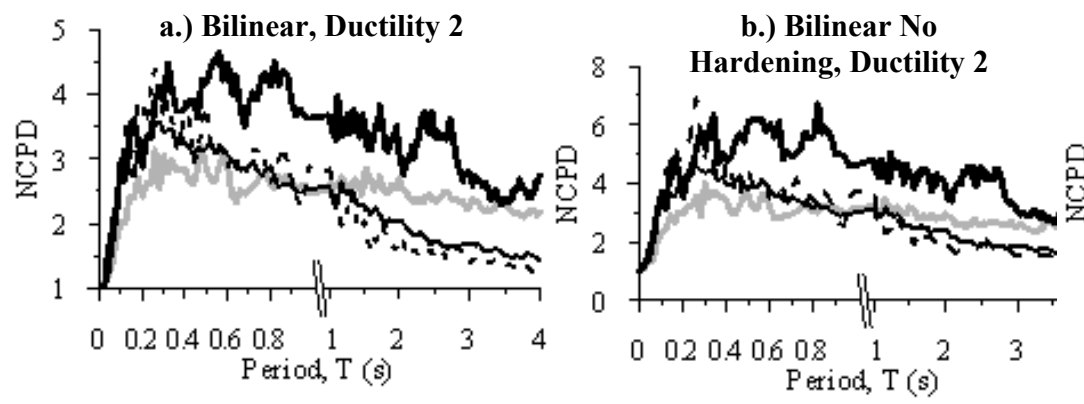

c.) Degrading, Ductility 2
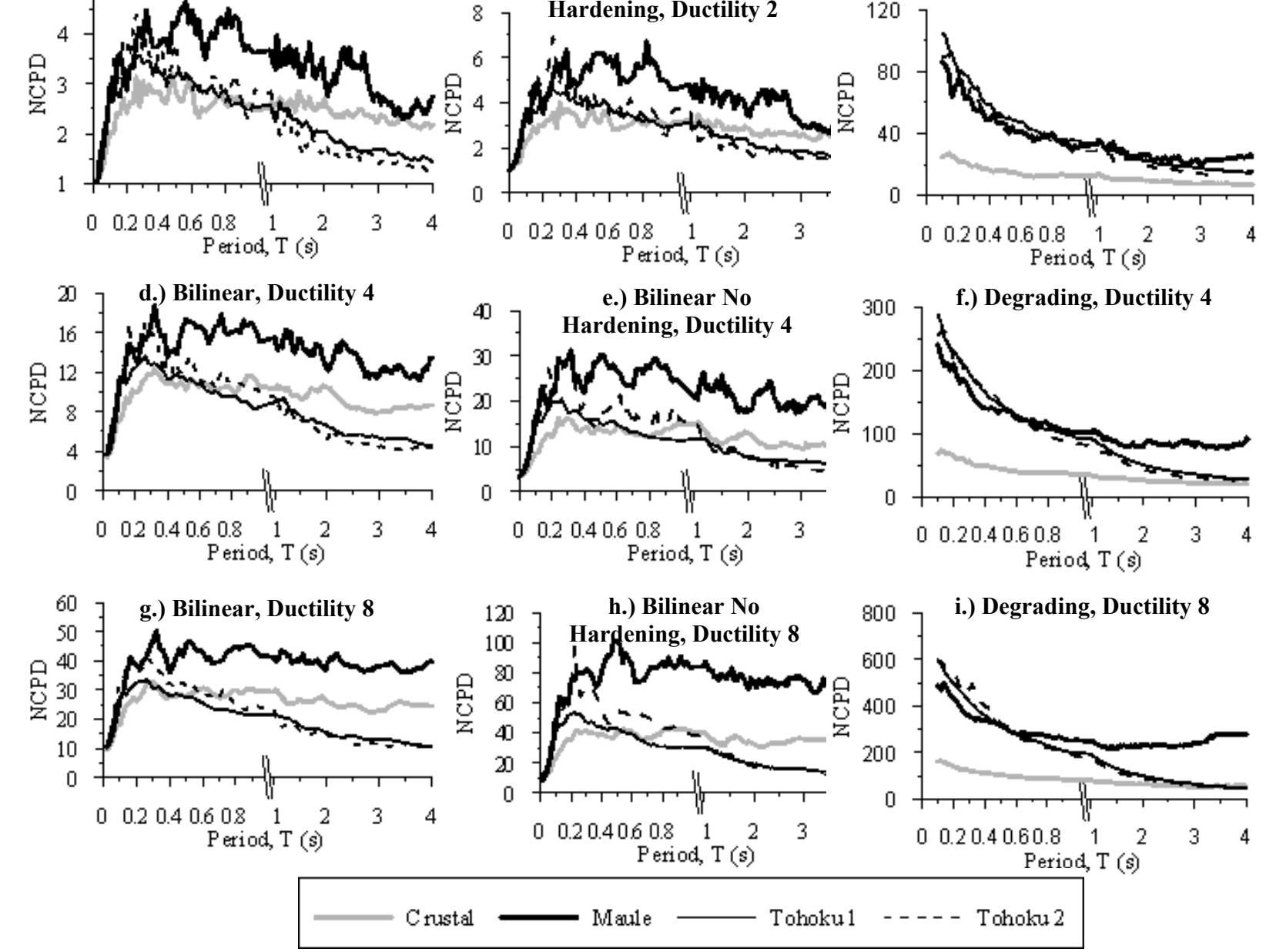

Figure 2.4: Mean NCPD Spectra: Bilinear and Degrading Models 

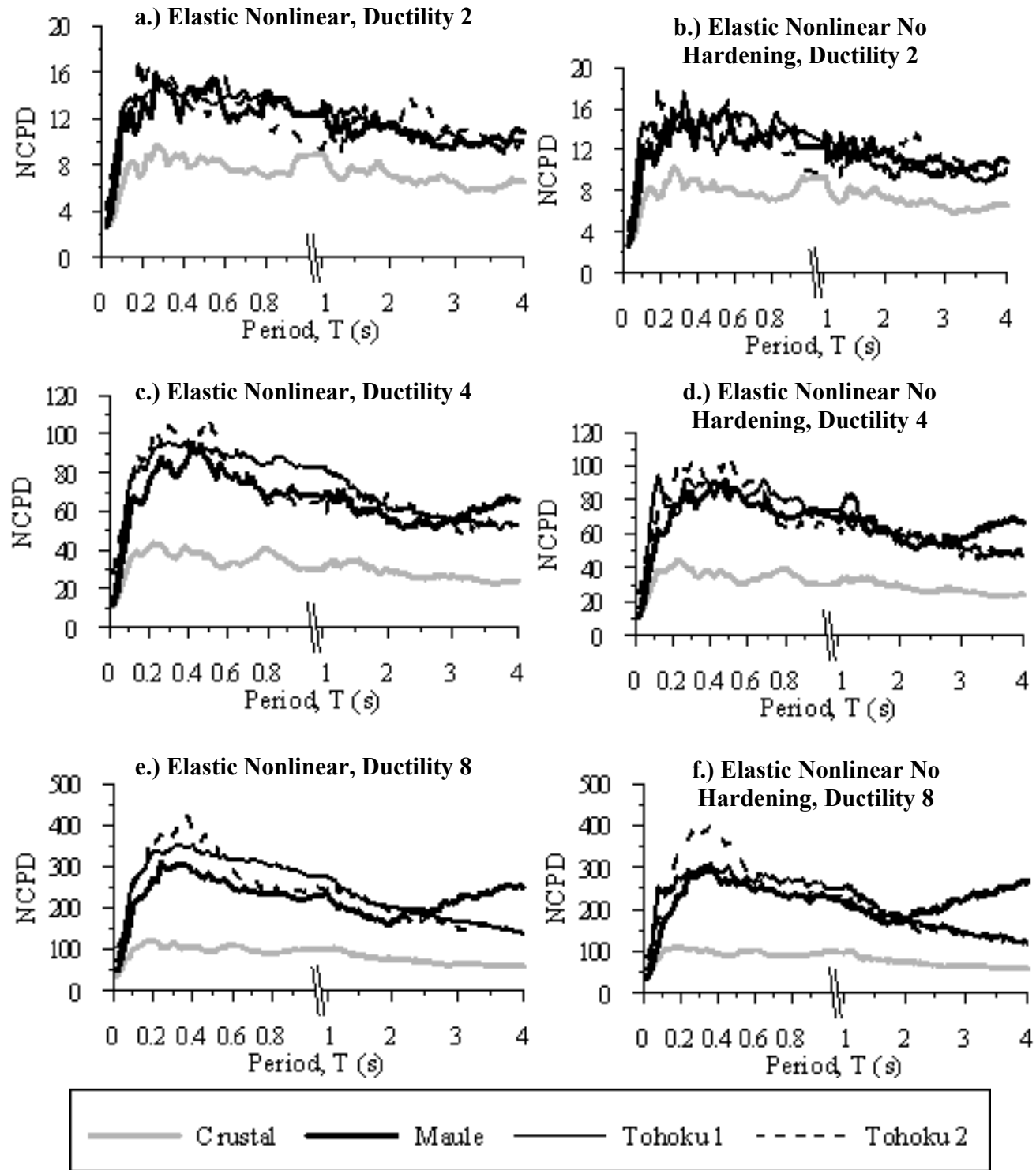

Figure 2.5: Mean NCPD Spectra: Elastic Nonlinear Model 


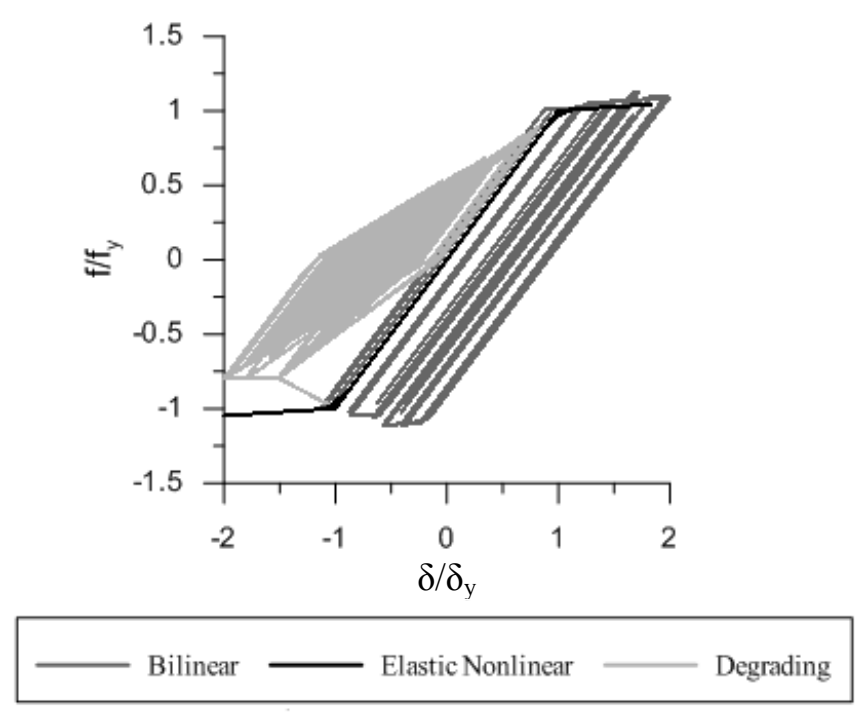

Figure 2.6: Example Maule Hysteresis, $\mathrm{T}=0.5 \mathrm{~s}$, Ductility 2

\subsubsection{Hardening Effects}

Both the elastic nonlinear and bilinear models were also analyzed without any post yield hardening. Because results were similar to those of the hardening counterparts, the full results were not included or discussed. However, an interesting effect was noticed. The trends for each model with and without hardening were very similar, including period effects and differences among ductility levels. In the case of the elastic nonlinear model, the difference in the magnitude of the demand with and without hardening was negligible. The magnitude of the demand of the bilinear model, however, was higher when hardening was not included on the order of approximately $25 \%$ to $80 \%$. Lower displacement demand is to be expected when hardening is introduced. The fact that this difference was only seen in the bilinear model suggests hysteretic damping is necessary for hardening to affect cumulative displacement demand.

\subsection{RESIDUAL DISPLACEMENT DEMAND}

Residual displacement was presented as an additional response metric. Because this was not a cumulative response metric, comparing the resulting trends led to conclusions about the importance of accounting for cumulative demands. This metric was calculated in a similar manner to NCPD. At each period of each SDOF system, the residual displacement was defined as the final displacement of each time history analysis. This value was then normalized by the yield displacement at that period of the system and presented as normalized residual displacement (NRD) spectra over the period range.

Similarly to inelastic acceleration response demand, the residual displacement demand did not appreciably vary among models. Results are shown only for the bilinear model in Figure 2.7 with a comparison among the models for the Maule set at ductility 2. For each level of ductility, the Tohoku sets resulted in higher residual displacement than the Maule and Crustal sets.

Furthermore, the Maule and Crustal sets resulted in very similar values of residual displacement. This was in contrast to the NCPD trends, where the Maule set consistently produced the highest 
cumulative displacement demand and the Tohoku2 sets produced results more similar to the Crustal set. The NRD results follow more closely what might be expected if PGA and acceleration spectra were used to predict damage. Here again, the need was apparent for a cumulative metric.
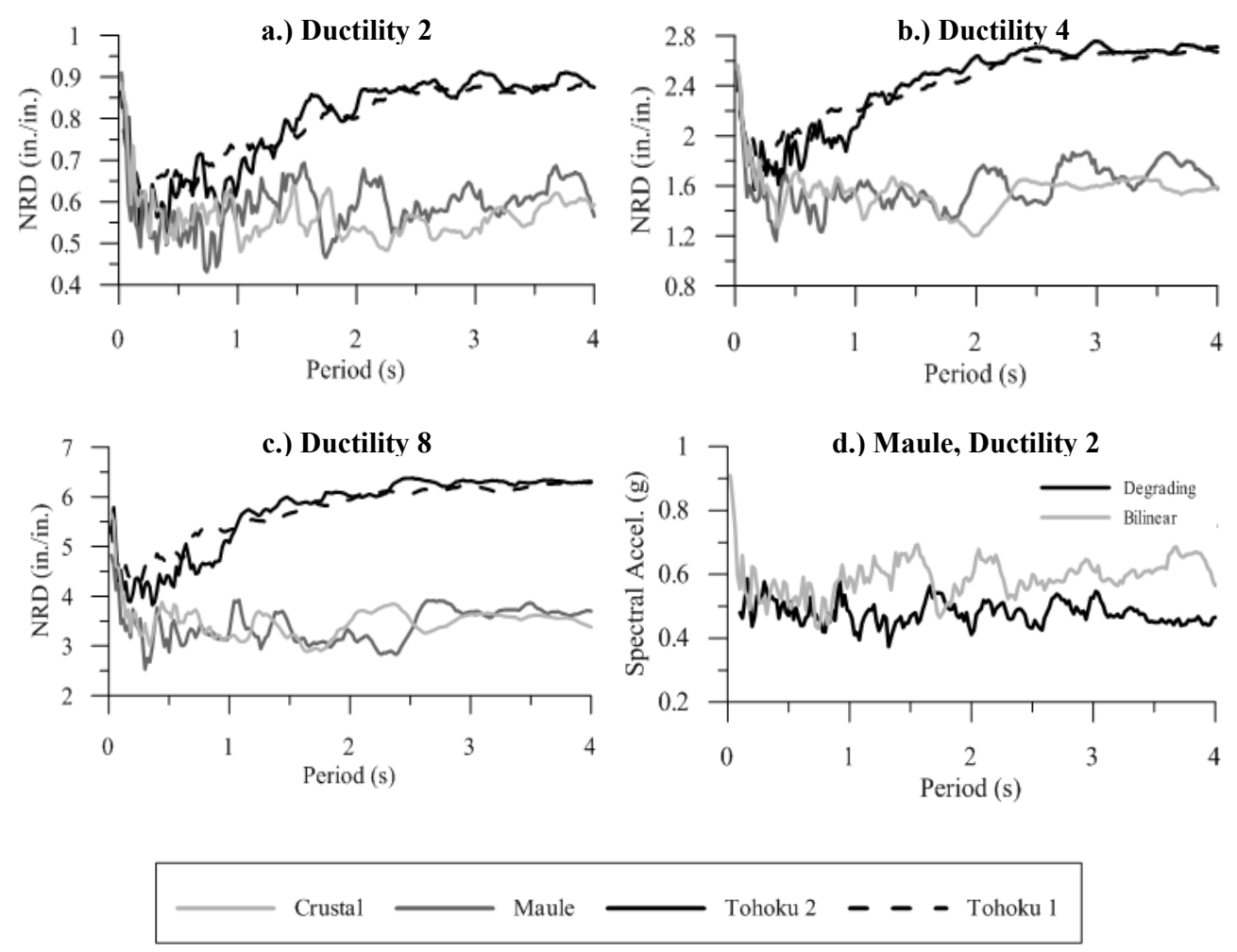

Figure 2.7: Mean NRD Spectra

\subsection{DURATION METRICS}

Previous researchers have looked at duration intensity measures, but most were based on characteristics of the ground motions themselves rather than the response of various structures to those ground motions. Two commonly used duration metrics are the "Bracketed Duration", and the "Normalized Arias intensity (NAI)". The bracketed duration in this study was taken as the first to the last occurrence of an acceleration of $0.05 \mathrm{~g}$ (Bolt 1969). The normalized Arias intensity was defined as the integral over the total record duration (tr) of the square of the acceleration time history as shown in Eq. (2.3) and normalized by PGA. The metrics were calculated and compared to the NCPD, and mean results for each set are shown in Table 2.1. The duration metrics would lead to similar expectations of demand as the inelastic acceleration content. Each indicated that the Tohoku1 would be expected to produce the highest demand. As this was not the case when cumulative duration was considered, the metrics are insufficient for capturing the expected damage due to duration effects. 


$$
N A I=\frac{\frac{\pi}{2 g} \int_{0}^{t r} a^{2}(t) d t}{P G A}
$$

Table 2.1: Mean Duration Metrics

\begin{tabular}{c|c|c|c|c}
\hline METRIC & TOHOKU1 & TOHOKU2 & MAULE & CRUSTAL \\
\hline Bracketed Duration $(\mathrm{sec})$ & 153 & 110 & 53 & 15 \\
\hline NAI $(\mathrm{g})$ & 169 & 107 & 123 & 42 \\
\hline
\end{tabular}

\subsection{COMPARISON OF TOHOKU 1 FULL SET AND SUBSET}

A subset of the Tohoku1 set was selected to represent the PGA range of the full set and approximate the number of earthquakes in the Maule and Crustal sets. Various results comparing the two sets are shown in Figure 2.8. As can be seen, the results do not considerably vary. Analysis was initially performed with all available ground motions in order to avoid any bias that might result from the method of ground motion selection. In this case, the selection process used to reduce the number of earthquakes in the set did not appreciably affect the results. This was most likely due to the fact that all ground motions under consideration were from the same earthquake and the reduced set maintained a statistically sufficiently large number of ground motions.
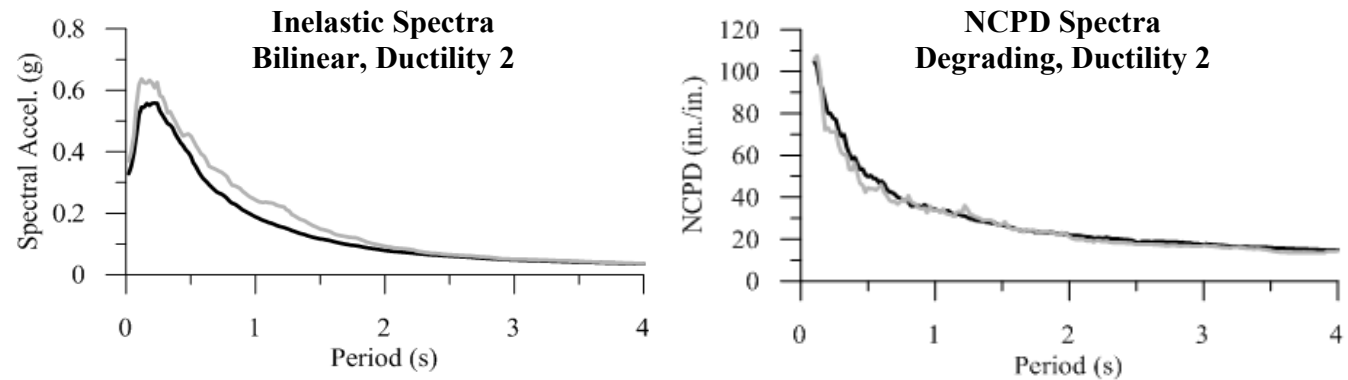

NCPD Spectra
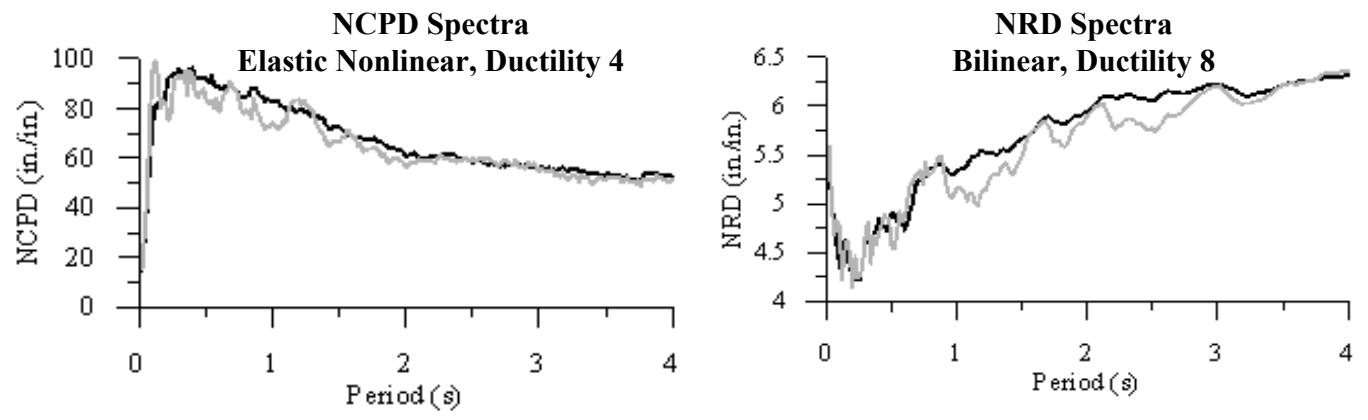

Tokoku1 Toholos 1 atset

Figure 2.8: Tohoku 1 compared to Subset 


\subsection{SUMMARY}

Observations based on this analysis can be summarized as follows:

- For all short period $(<\sim 0.5 \mathrm{~s})$ structures, subduction earthquakes produced more cumulative displacement than crustal earthquakes.

- The Maule earthquake produced at least as much cumulative displacement, in most cases more, than the Tohoku earthquake particularly at longer periods $(>\sim 1.0 \mathrm{~s})$ despite having shorter duration.

- Resulting cumulative displacement did not conform to the levels of damage that would be expected based on other more common intensity measures including simply looking at earthquake record duration and response metrics.

- Structures with hardening accumulated less displacement than structures without hardening, but only when hysteretic damping was also present. Hardening did not affect the accumulated damage in elastic nonlinear structures.

- Reducing the number of ground motions in an analysis set from 166 to 34 did not appreciably alter the results, and would most likely not do so for any case where all ground motions are from the same earthquake source. 


\subsection{CYCLIC LOADING PROTOCOL CONSIDERING SUBDUCTION MEGA EARTHQUAKES}

\subsection{GENERAL}

All structural components have limited capacity. For that reason, understanding their behavior under strong ground motion excitations has always been a major objective of earthquake engineering. One method to assess the performance of structural components is via experimental evaluations utilizing quasi-static cyclic loading. The relatively slow application of the load in quasi-static tests allows experimentalists to relate structural metrics such as top displacement, chord rotation, drift, strains, etc. to visual damage of specimens (e.g. first cracking, spalling of the concrete, buckling of longitudinal reinforcement). Current earthquake design procedures for structural components have been established based on experimental results utilizing quasi-static cyclic tests. Moreover, design codes are trending to a relatively new design methodology called "Performance-based seismic design" (PBSD). In this methodology, a number of performance levels, which are frequently defined in terms of acceptable levels of damage, need to be satisfied under different levels of seismic hazards. Under this design methodology the assessment of different structural components plays a fundamental role.

Numerous experimental and analytical studies have been conducted in order to assess structural components, define limit states and acceptance criteria to be used in performance-based seismic design (Hose and Seible 1999, FEMA356 2000, ASCE/SEI41-06 2007). Many protocols have been developed and utilized to assess structural and non-structural components, e.g. Krawinkler et al. (Krawinkler et al. 1983), Cheung et al. (Cheung et al. 1991), ATC-24 (ATC-24 1992), Krawinkler et al. (Krawinkler et al. 2000a, 2000b), Richard and Uang (Richard and Uang 2006), FEMA461 (FEMA461 2007), Hutchinson and Wood (Hutchinson and Wood 2013), ACI374.2R13 (ACI374.2R-13 2013). Despite these efforts, past loading protocol developments had not considered subduction ground motions because a scarcity of this type of record. However, the recent occurrence of highly devastating subduction megathrust earthquakes of long duration, 2010 Maule, Chile and 2011 Tohoku, Japan, have raised researchers' interest in how earthquake duration and number of cycles affect structural response, collapse assessment and overall performance of structural components subjected to subduction zone earthquakes. The occurrence of these seismic events suggests that large magnitude ground motions of long duration have the potential of significantly increase the number of inelastic excursions and consequently incur more extensive structural damage compared to ground motions with similar elastic spectral displacement demands but less duration as discussed in Chapter 2, and also in Raghunandan and Liel (Raghunandan and Liel 2013), and Chandramohan et al. (Chandramohan et al. 2013). This effect is mostly attributed to the rate of structural strength and stiffness deterioration due to an increase in load reversals imposed for large magnitude and long duration ground motions. This aspect is particularly relevant in subduction zones due to the fact that larger magnitude earthquakes are associated with strong motions of long duration (Dobry 1978), (Midorikawa et al. 2012). The increase of inelastic demands creates a critical necessity to improve current 
loading protocols, which predominantly have been developed using crustal ground motions of moderate to high magnitude.

Capturing the mega subduction effect is of vital importance in the assessment of RC bridge components located in the Pacific Northwest coast of the United States which lies near the Cascadia subduction zone and where a mega thrust earthquake of long duration forms a major component of the seismic risk. For that reason, in this chapter is discussed the development of a rational quasi-static loading protocol capable of simulating the demands imposed on reinforced concrete bridge columns.

In order to achieve this objective the results from non-linear time history analyses considering numerous subduction ground motions imposed on structures with a wide range of structural periods and pre-determined ductilities were analyzed using a simplified rainflow counting procedure (ASTM E1049-85 2005). Throughout the analyses and development, a methodology similar to the one utilized for the development of a testing protocol for wood frame structures (Krawinkler et al. 2000a) was utilized. Since strength and deformation capacities of structural components depend on the cumulative damage that the system undergoes under load reversals imposed by seismic excitations. It is recognized a direct relationship between the number of inelastic cycles and damage (Krawinkler et al. 1983), (Stephens \& Yao 1987). Various models have been developed to quantify this relationship (Cosenza et al. 1993) (Ghobarah et al. 1999); two well-known relationships describing the cumulative damage are the normalized cumulative ductility and the Coffin-Manson law for low-cycle fatigue in conjunction with the Miner's rule of linear damage accumulation. Both models show a direct relationship between structural damage and the number and amplitude of damaging cycles. Thus, the number of inelastic cycles and a cumulative damage demand were selected as the main parameters to develop the protocols. A range of protocols was developed with the aim of capturing more closely the inelastic demands of subduction megathrust earthquakes and, consequently, improving the seismic assessment of bridge columns through physical testing.

\subsection{IMPLICATIONS FROM PAST RESEARCH}

Limited experimental data can be found on reinforced concrete (RC) columns subjected to long duration protocols that try to simulate subduction zone earthquakes since most of the seismic assessment of RC bridge columns has been carried out using conventional cyclic loading protocols, such as those shown in Figure 3.1. These conventional protocols have been developed to reflect seismic cumulative demands of short period structures that are not representative of long period structures (Cheung et al. 1991), (Priestley et al. 2002), (ACI 374.2R-13 2013) and do not represent the demands imposed by subduction mega earthquakes.

Moreover, experimental studies have shown that the displacement capacity of structural components is influenced by the loading history applied. A relevant research was carried out by Takemura and Kawashima (Takemura and Kawashima 1997) to study the influence that different loading histories have on the ductility capacity of reinforced concrete bridge piers. In Takemura's research six nominally identical specimens were tested under different loading protocols resulting in six different responses. Another relevant research was carried out by Kunnath, et al. (Kunnath 1997) to investigate the cumulative seismic damage in circular reinforced concrete bridge columns, which were mostly controlled by flexural behavior. With 
that purpose, twelve columns were tested to quarter-scale. Test results led to the conclusion that the load path highly influences the failure mode of RC columns. In this study was found that columns subjected to cycles of low amplitude were likely to present a failure in the confinement rather than in the longitudinal bars. On the other hand, under cycles of high amplitude the failure mode was controlled by rupture in the longitudinal reinforcement. The study also revealed that conventional loading protocols commonly used in experimental testing tend to replicate unrealistic drift demands because numerous large inelastic reversals are imposed in the component. This is not representative of typical earthquakes demands, which usually imposed several inelastic cycles of low amplitude and just a few large inelastic cycles.

Similarly, using the concept of low-cycle fatigue and the cumulative damage model employed in the research carried out by Kunnath, experimental tests were performed at the Washington State University in order to investigate the performance of pre-1975 concrete bridges subjected to subduction earthquakes (McDaniel et al. 2006). In this research, eight circular lightly confined reinforced concrete columns were tested using different displacement history to represent the demands imposed by subduction zone ground motions of long duration. Loading protocols with cycles of constant amplitude were performed to simulate those demands. The columns showed three different modes of failure depending on the load protocol employed. The first mode of failure was an interaction of shear and flexure when the applied protocol consisted of large initial inelastic cycles; the second failure mode was buckling of the longitudinal reinforcement when the column was subjected to many small inelastic cycles. The final mode of failure was slipping of the longitudinal reinforcement under a considerable amount of small amplitude inelastic cycles followed by large ones. Thus, these results, as well as those obtained by Kunnath (Kunnath 1997), showed that the failure mode of RC columns depends on the displacement history applied to them.

Moreover, Pujol (Pujol 2006) and Borg (Borg 2012) carried out experimental studies of reinforced concrete columns subjected to different loading histories, which showed that the drift capacity is highly influenced by the displacement history and the number of cycles.

A similar study was recently performed at MCEER, University at Buffalo in conjunction with the National Taiwan University of Science and Technology (Ou et al. 2013). In this case, reinforced concrete bridge columns were tested applying two different loading protocols to investigate the influence of the number of cycles on bridge columns. Test results showed that columns under a long duration protocol behave significantly different in terms of strength and stiffness degradation than those columns under conventional protocols showing that on high levels of damage the strength and stiffness degradation of the specimen would increase markedly under long duration earthquakes of large magnitude.

Thus, recent research studies in conjunction with the occurrence of devastating subduction zone mega earthquakes demonstrates that the development of loading protocols reflecting the increase in the number of inelastic demands posed by ground motions of large magnitude and long duration is needed to improve the assessment of RC bridge columns through experimental evaluations. 


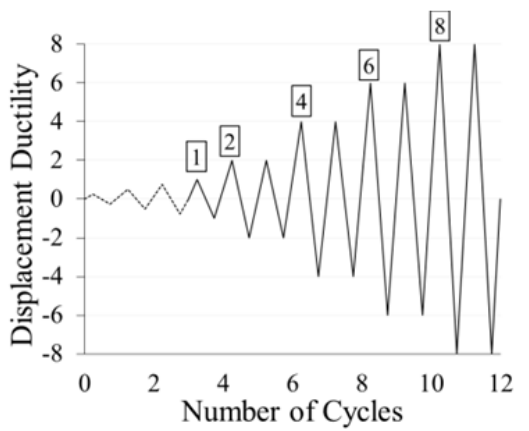

(a)

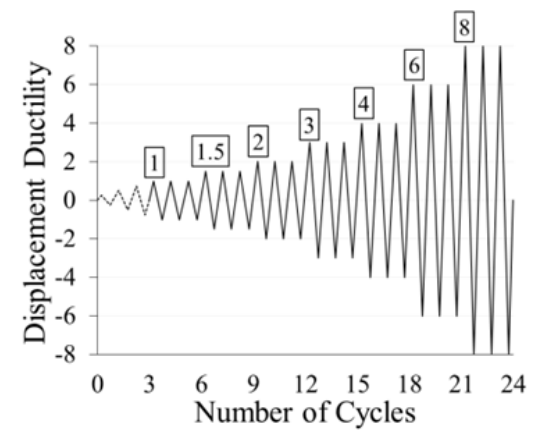

(b)

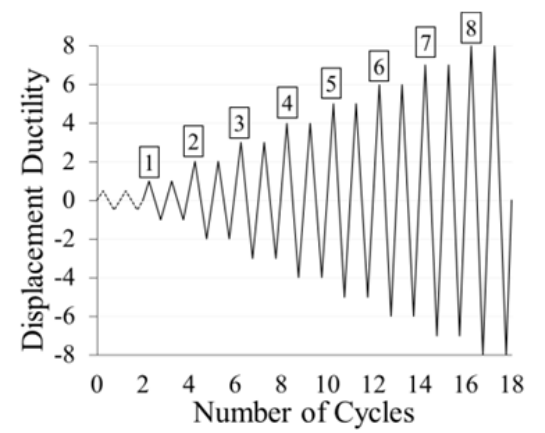

(c)

Figure 3.1: Conventional loading protocols used on RC column assessment. (a) New Zealand Protocol (Cheung et al. 1991), (b) Modified New Zealand Protocol (Priestley et al. 2002), (c) ACI374 unidirectional protocol (ACI 374.2R-13 2013).

\subsection{SELECTION OF EARTHQUAKE GROUND MOTIONS}

Regions of the Pacific Northwest Coast of the United States, which lies near the Cascadia subduction zone (also referred to as the Juan the Fuca subduction zone), may be affected by a megathrust earthquake of long duration similar to those occurred in Chile and Japan (Heaton and Hartzell 1986). Most of the loading protocols used in seismic assessment have been developed for a specific structural or nonstructural component by utilizing a set of crustal ground motions. These ground motions are often representative of the $10 \%$ probability of exceedance in 50 years $(10 / 50)$ hazard level for Los Angeles conditions and were selected to avoid near-fault effects, at distances from fault rupture greater than $13 \mathrm{~km}$, and from crustal earthquakes of moment magnitudes $\left(\mathrm{M}_{\mathrm{w}}\right)$ varying between 6.7 and 7.3. (Krawinkler et al. 2000a), (FEMA 461 2007)

With the aim of developing representative loading protocols for bridge components, a selection of strong motion records similar to that presented in Chapter 2 was conducted in order to determine the inelastic demands imposed by subduction megathrust earthquakes as shown in Table 1. The subduction zone ground motion sets used in the development of loading protocols were chosen from the 1985 Valparaiso (COSMOS 2012), 2007 Sumatra (COSMOS), 2010 Maule (U. Chile 2010), and 2011 Tohoku (K-Net undated) earthquakes with distances to the epicenter greater than $100 \mathrm{~km}$ to reduce the number of records and ensure far field response. The subduction ground motion sets, in spite of being treated as a single set called Subduction, were divided into four sub-sets in order to observe the differences in the inelastic demands that could be generated by variations in earthquake characteristics or regional geology. Tohoku ground motions were further differentiated, referred to herein as Tohoku1 and Tohoku 2, because of the large amount of records available on the Kyoshin Network Database (K-Net undated) and in order to have one of the Tohoku sets with similar PGA range to the other sets. Only one pair of ground motions for the 2007 Sumatra earthquake was utilized due to the lack of strong motion records available.

A set of crustal ground motions was utilized to allow for demand comparisons. Crustal ground motions, referred to herein as Crustal set, were chosen from the FEMA P695 far-field record (FEMA P695 2009), which is based on a representative set of twenty-two horizontal ground 
motions taken from the PEER database (PEER 2006) with the following criteria: from sites located at distance greater than $10 \mathrm{~km}$ from the fault rupture, moment magnitude greater than 6.5, recorded from soils categorized as Site Class C and D, and originated from shallow crustal sources (strike-slip or reverse fault mechanisms). The number of strongest records was limited from each earthquake to two and thus avoids bias in the results.

For each ground motion recording two orthogonal horizontal records were treated separately, while the vertical ground motion components were not considered. Although, there is no general consensus on the definition and determination of the strong ground motion duration, in this study the duration was taken as the "Bracketed Duration", which is defined as the first to the last occurrence of an acceleration of $0.05 \mathrm{~g}$ (Bolt 1969). The average duration of subduction ground motions was found to be at least twice that of the crustal set as summarized in Table 3.1. Furthermore, the bracketed duration for the Tohoku sets is from 7 to 10 times higher than the duration for the crustal set. In this study, no scaling procedure was employed on the records and instead a target ductility design was conducted as discussed in Chapter 2. In this manner, the earthquake source variability was minimized since recent studies have demonstrated that scaling input records to a target spectral acceleration level $\left(\mathrm{S}_{\mathrm{a}}\right)$ can produce biased results (Luco and Bazzurro 2007).

Table 3.1: Ground motion sets used for the development of cyclic loading protocols

\begin{tabular}{|c|c|c|c|c|c|c|}
\hline Set & $\mathbf{M}_{\mathbf{w}}{ }^{1}$ & $\begin{array}{l}\text { Site } \\
\text { Class }\end{array}$ & $\begin{array}{l}\text { Dist. Epicenter } \\
\text { Average (km) }\end{array}$ & $\begin{array}{c}\text { PGA } \\
\text { Range (g) }\end{array}$ & $\begin{array}{c}\text { Number of } \\
\text { Records }\end{array}$ & $\begin{array}{c}\text { Average } \\
\text { Bracketed } \\
\text { Duration (sec) }\end{array}$ \\
\hline Crustal & $6.5-7.6$ & $\mathrm{C} / \mathrm{D}$ & 39 & $0.21-0.82$ & 37 & 15 \\
\hline Valparaiso & $7.8^{2}$ & $\mathrm{~B} / \mathrm{D}$ & 137 & $0.11-0.71$ & 36 & 39 \\
\hline Sumatra & 7.9 & - & 168 & 0.13 & 2 & 48 \\
\hline Maule & 8.8 & $\mathrm{~B} / \mathrm{D}$ & 357 & $0.13-0.93$ & 31 & 53 \\
\hline Tohokul & 9.0 & $\mathrm{~B} / \mathrm{C} / \mathrm{D}$ & 238 & $0.94-2.01$ & 27 & 153 \\
\hline Tohoku2 & 9.0 & $\mathrm{D} / \mathrm{E}$ & 282 & $0.20-0.81$ & 166 & 110 \\
\hline
\end{tabular}

\subsection{LOADING PROTOCOL DEVELOPMENT}

\subsubsection{Hysteresis Model and Target Response}

The main objective of a loading protocol is to assess the structural capacity of components. Since demand and capacity are not independent, it is reasonable to think that one should know the seismic excitation imposed on the component and its response before the development of any protocol.

In order to predict the damage that a structure undergoes during severe earthquakes, it is important to represent in a realistic way the behavior of structural components during loading reversals. In the case of reinforced concrete components, the Clough (Clough 1966) and Takeda (Takeda et al. 1970) hysteretic models are widely used. However, these models assume that the stiffness degradation is related to the maximum displacement of the system and not to the 
number of cycles. Since the analyses were expected to incorporate numerous large inelastic excursions, the peak oriented Ibarra-Medina-Krawinkler hysteretic model (Ibarra et al. 2005a) was utilized. This model was referred to as "Degrading model" in Chapter 2 and includes strength capping, residual strength, and strength and stiffness deterioration caused by load reversals as illustrated in Figure 3.2. This model was calibrated using test results of bridge columns dominated by flexural behavior. Column tests can be found in the Pacific Earthquake Engineering Research Center (PEER) structural performance database. This process allowed us to find appropriate parameters to closely simulate load-deformation behavior of the components in study.

Results from nonlinear time-history analyses of single degree of freedom systems (SDOF) using OpenSees (OpenSees 2011), as described in Chapter 2, were utilized. In OpenSees ach SDOF system was modeled as a zero length element. The model parameters were calibrated using test results of bridge columns dominated by flexural behavior. A damping ratio of $5 \%$ was set for the analysis. A wide range of structural fundamental periods were considered from 0.2 to 4.0 seconds by maintaining the mass of the system constant and varying the stiffness of the SDOF system. Since the majority of modern seismic design codes for bridges (AASHTO 2009), (Caltrans 2013) rely on component ductilities, a constant ductility inelastic response approach (Ridell and Newmark 1979), (Krawinkler 1996) was deemed suitable to perform the nonlinear analyses. This approach assumes that for each ground motion and at each period, the structural system is designed to reach the pre-determined ductility $(\mu)$ by finding the yield strength of the structure that produced the desired ductility value. Ductility is defined as the ratio of the maximum displacement to the yield displacement $\left(\mu=\delta_{\mathrm{u}} / \delta_{\mathrm{y}}\right)$, where the maximum ductility capacity was calculated when the force in the post-capping range degraded to not less than 80 percent of the maximum as illustrated in Figure 3.2. Consequently, recursive analyses were needed to design each system in order to reach pre-determined displacement ductilities of 2, 4 and 8 .

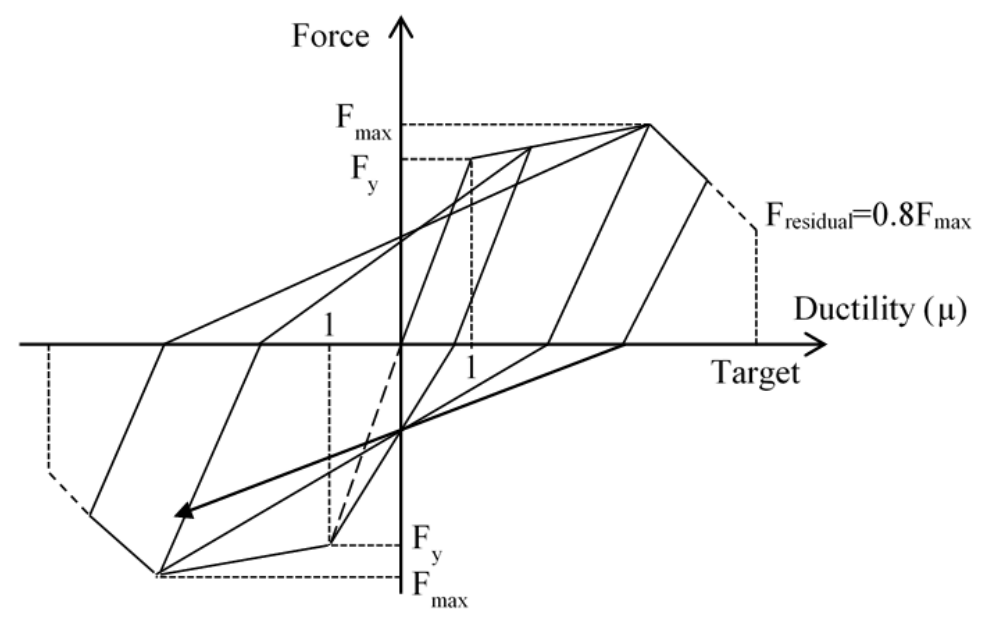

Figure 3.2: Hysteretic behavior of SDOF system. Adapted from Ibarra et al. (Ibarra et al. 2005a) 


\subsubsection{Cumulative Damage}

Strength and deformation capacities of structural components depend on the cumulative damage that the system undergoes under load reversals imposed by seismic excitations. A direct relationship between the number of inelastic cycles and damage can be assumed (Krawinkler et al. 1983), (Stephens and Yao 1987). Various models have been developed to quantify this relationship (Cosenza et al. 1993) (Ghobarah et al. 1999). In order to capture the behavior under ground motion excitations, most of current testing protocol developments and experimental studies have been conducted based on a general cumulative damage concept using the CoffinMason model and the Miner's rule of linear damage accumulation as a baseline (Krawinkler et al. 1983). This concept implies that every excursion in the inelastic range will cause damage in the component, and this damage will be accumulated from excursion to excursion, where an excursion is defined as the path from one peak to the next peak in the time history response. Thus, the performance of structural components depends on the previous inelastic excursions alluding to a memory of past damaging events. In this approach, the damage is directly affected by the number of inelastic excursions $(N)$, the range of each plastic excursion $\left(\Delta \delta_{\mathrm{pi}}\right)$, and the sum of those ranges as shown in Eq. (3.1). C and $\mathrm{c}$ are structural performance parameters that depend on the type of component and failure mode. The parameter $\mathrm{c}$ is usually greater than 1.0 , which implies that large inelastic excursions cause more damage than low ones. This damage index has demonstrated good correlations between the quantitative and descriptive damage for steel structures (Krawinkler et al. 1983) and reinforced concrete structures (Kunnath et al. 1997).

$$
D=C \sum_{i=1}^{N}\left(\Delta \delta_{p i}\right)^{c}
$$

Another extensively damage index used in reinforced concrete structures was formulated by Park and Ang (1985), which accounts that damage is caused by the maximum deformation and the cumulative dissipated energy as shown in Eq. (3.2).

$$
D_{p a}=\frac{\delta_{\max }}{\delta_{u}}+\frac{\beta}{Q_{y} \delta_{u}} \int d E
$$

Where $D_{p a}$ is the damage index; $\delta_{\max }$ is the maximum response deformation; $\delta_{u}$ is the ultimate deformation capacity under static load (monotonic response); $Q_{y}$ is the calculated yield strength; $d E$ is the incremental dissipated hysteretic energy; and $\beta$ is a factor that depends on several structural parameters that try to measure the duration effect on the hysteretic energy.

However, in order to calculate the damage indices $D$ and $D_{p a}$, in a meaningful way, the parameters $\mathrm{c}, \mathrm{C}$ and $\beta$ have to be experimentally obtained and validated, which can lead to undesirable uncertainties in the development of the protocol since those parameters are directly influenced by the cyclic loading history. For this reason, in this study was utilized another damage index also based on cumulative damage called Normalized Cumulative Plastic Displacement (NCPD), which is a simple measure of structural deterioration. This metric is calculated by accumulating the ratio of plastic displacement range under an excursion $\left(\Delta \delta_{\mathrm{pi}}\right)$ to the yield displacement $\left(\delta_{\mathrm{i}}\right)$ as shown in Eq. (3.3). 


$$
N C P D=\sum_{i=1}^{N} \frac{\Delta \delta_{p i}}{\delta_{y}}
$$

The number of damaging cycles $(\mathrm{N})$ and the NCPD were considered the target demand parameters in the development of testing protocols for RC bridge columns. In this sense, a cycle is considered damaging when its amplitude is greater than the yield displacement.

\subsubsection{Cycle Counting}

The seismic response exhibited by a structural component contains excursions that are not symmetric, and does not follow a consistent pattern under different ground motions. Additionally, sequence effects, which are related to the fact that not all the inelastic excursions occur before the maximum one, have not been fully established through analytical and experimental studies. In order to overcome this sequence effect in the development of loading protocols for structural components, researchers have been using the concept of pre-peak excursions (Krawinkler et al. 2000a), (FEMA 461 2007). Understanding as pre-peak excursion any excursion that occurs before either the maximum or minimum peak displacement. Using all excursions may lead to an overestimation of cumulative damage because most of the damage sustained by a structural component occurs when excursions tend to widen the hysteresis response. The development of the loading protocols in this study was primarily based on prepeak excursions. However, given the long duration of the subduction records, both pre-peak as well as all excursions were considered for comparison purposes in order to analyze the implications of selecting one over the other.

To rationalize the development of the testing protocol and compare the demands imposed by different ground motions, the time history responses based on pre-peak excursions were converted into a series of cycles using the simplified rainflow counting methodology (ASTM E1049-85 2005) as illustrated in Figure 3.3. This method of cycle-counting results in a deformation history composed only of full cycles due to the fact that the history response is rearranged in a way that always starts with either the maximum or minimum peak. The resultant excursions and cycles obtained after performing the rainflow counting are not symmetric with respect to the undeformed condition, also referred to as the mean effect, which could distort the cumulative damage of the structure. However, this effect is not considered in many practical cases because the effect of large mean deformations primarily influences the response in situations of near fault rupture (Krawinkler et al. 2000b). Thus, the proposed displacement protocols were developed with a stepwise increasing deformation of symmetric excursions instead of asymmetric excursions. The rainflow counting procedure was employed in the nonlinear time history response of individual SDOF systems across the range of fundamental periods. This generated a vast amount of data, which was statistically reduced to allow for comparisons in a rational way. The data that was extracted and post-processed from the rainflow counting procedure was the number of inelastic cycles and the NCPD, where the number of inelastic cycles was equal to the number of cycles above ductility one $(\mu=1.0)$ as shown in Figure 3.3(d) and the NCPD was obtained by using Eq. (3.2). It is worth mentioning that the NCPD computed for the development of cyclic loading protocols differs from that shown in Chapter 2 since the former was obtained as a result of the rainflow counting procedure. The number of inelastic cycles and the NCPD were represented employing the 84th percentile as 
target value in order to represent a reasonable and conservative estimate of the demand parameters, and also in an effort to bound the applicability of the resulting protocols.
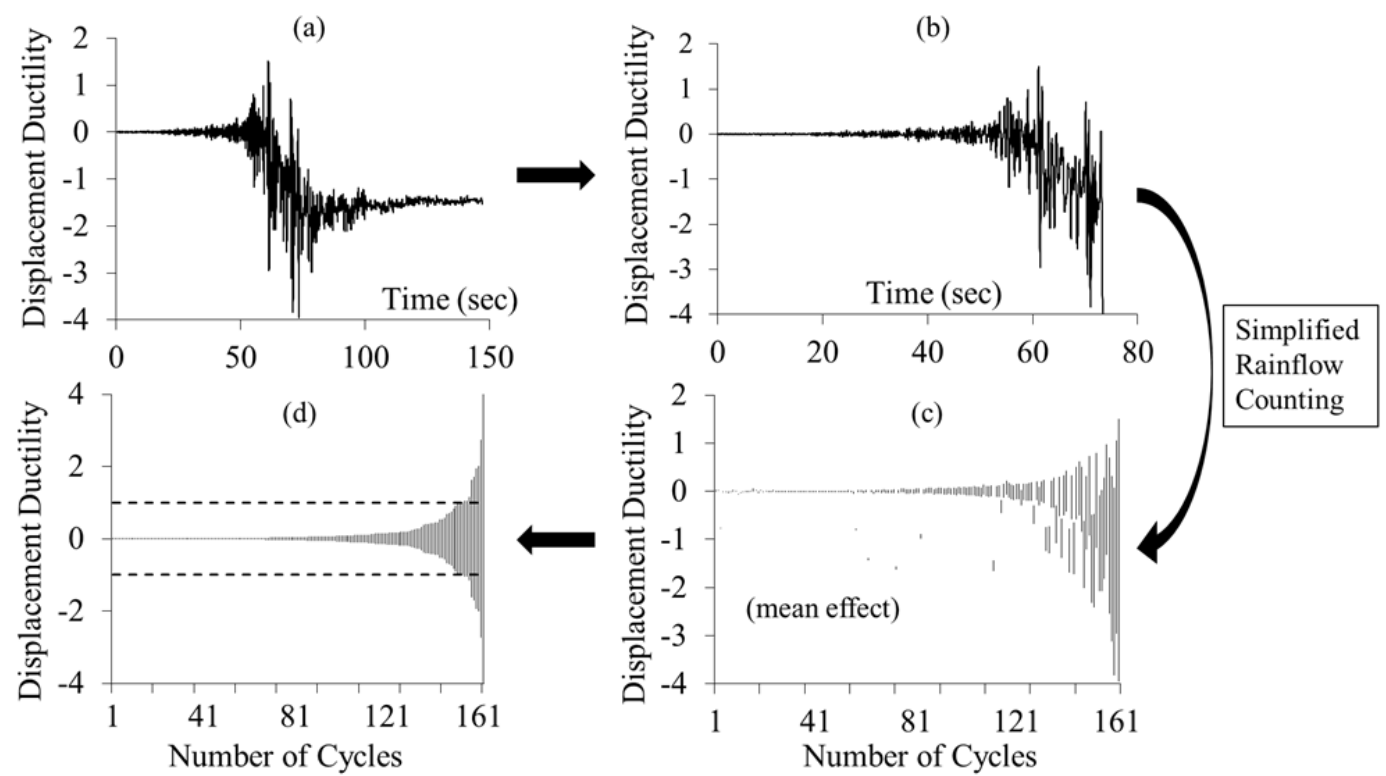

Figure 3.3: Illustration of the simplified rainflow counting procedure. (a) Inelastic response history. (b) Pre-Peak inelastic response history. (c) Ordered cycles including mean effect. (d) Final ordered cycles.

\subsubsection{Results and Protocol Development}

Results from the three pre-determined ductilities and a wide range of structural fundamental periods were considered in the development of the protocols in order to represent a vast number of bridge structures. Moreover, the results obtained by using structures of different fundamental periods was specially considered since conventional protocols commonly used to assess the seismic performance of $\mathrm{RC}$ structures were developed to reflect demands on short period structures (FEMA 461 2007), which are not often representative of bridge responses. From these results, the number of inelastic cycles and NCPD showed a high dependence on the fundamental period, as illustrated in Figure 3.4 and Figure 3.5. The figures represent the $84^{\text {th }}$ percentile of each set and clearly trend toward reduced demand with increase in period. Furthermore, the $84^{\text {th }}$ percentile of all the subduction records were utilized to develop the protocols since the results of each set of subduction ground motion showed a similar trend for structures with periods less than $2.0 \mathrm{sec}$. The demand parameters also showed that for structures with periods less than $2.0 \mathrm{sec}$ the demands imposed by subduction earthquakes are on average 100\% higher than those for crustal earthquakes. For periods above $2.0 \mathrm{sec}$, the results depended on the earthquake set. This effect is most evident for the Chilean earthquakes (Valparaiso and Maule sets) where the number of inelastic cycles and the NCPD tend to plateau significantly above the crustal sets for fundamental periods over $2.0 \mathrm{sec}$. Meanwhile, the same parameters for the Indonesia and Tohoku sets tend to decrease with increasing period, approaching the demands under the action of the Crustal set. The results led to the conclusion that cumulative plastic demands in bridges of long fundamental periods ( $\geq 2.0 \mathrm{sec}$ ) imposed by Chilean earthquakes (Maule and Valparaiso), are larger than those imposed by Tohoku and Indonesia earthquakes. This effect is more predominant for higher 
ductilities and when all the excursions are considered. The number of inelastic cycles and the NCPD decrease as the period of the system increases, which implies that the critical demand occurs for structures with low fundamental period. Multi span bridges that rely on columns in addition to abutments are unlikely to exhibit such short fundamental periods. For those reasons, three fundamental periods of $0.5,1.0$ and 2.0 seconds were selected for cyclic load protocol development as a benchmark representation of expected bridge fundamental periods, referred to herein as short $(0.5 \mathrm{~s})$, medium $(1.0 \mathrm{~s})$ and long $(2.0 \mathrm{~s})$ fundamental periods. The consideration of different natural periods reflects the period dependency of the analysis results. Appropriate selection of resulting protocols may avoid overestimation of inelastic cycles that the structure undergoes or distortion in the assessment of the behavior during physical testing.

The influence of different target ductilities can also be compared. Increase in achieved ductility increases both the number of inelastic cycles $(\mathrm{N})$ and the normalized cumulative plastic displacement (NCPD). For the benchmark periods averaged over all sets, results have shown a nearly linear relation in the NCPD for different ductilities as illustrated in Figure 3.6, which implies that for structures with other ductilities the cumulative demand may be found by linear interpolation of the values for ductilities 2,4 , and 8 . On the other hand, the number of inelastic cycles does not show a linear relation as the gradient reduces with increased period. This trend implies that linear interpolation between the selected benchmark periods would result in conservative estimate of inelastic cycles and could be a practical choice for protocol selection between the representative target ductilities used in this paper.

Comparison of pre-peak and all excursion demands is summarized in Table 3.2 using a relative ratio. For structures of low ductility $(\mu=2)$ pre-peak excursions represent over $75 \%$ of the demands computed considering all excursions. Identical protocols, for both pre-peak and all excursions, were proposed for low ductile structures since the demands between the two approaches were similar. On the other hand, the ratios between pre-peak excursion demands and all excursion demands decrease as the ductility of the structure increases, with ratios as low as $63 \%$ in the case of high ductile structures $(\mu=8)$. Therefore, using all excursions in the development of testing protocols may lead to more damage in moderate and high ductility structures.

Table 3.2: Comparison of demand parameters for pre-peak and all excursions

\begin{tabular}{c|c|c|c}
\hline \multicolumn{4}{|c}{ Ratio Pre-Peak/All Excursions } \\
\hline \multirow{2}{*}{$\begin{array}{c}\text { Period } \\
\mathrm{T}\end{array}$} & $\begin{array}{c}\text { Max } \\
\mu\end{array}$ & $\mathrm{N}_{\text {cycles }}>\delta_{\mathrm{y}}$ & \multirow{2}{*}{ NCPD } \\
\hline \multirow{3}{*}{0.5} & 2 & $78 \%$ & $80 \%$ \\
\cline { 2 - 4 } & 4 & $74 \%$ & $75 \%$ \\
\cline { 2 - 4 } & 8 & $66 \%$ & $69 \%$ \\
\hline \multirow{3}{*}{1.0} & 2 & $75 \%$ & $77 \%$ \\
\cline { 2 - 4 } & 4 & $70 \%$ & $72 \%$ \\
\cline { 2 - 4 } & 8 & $67 \%$ & $70 \%$ \\
\hline \multirow{3}{*}{2.0} & 2 & $83 \%$ & $85 \%$ \\
\cline { 2 - 4 } & 4 & $71 \%$ & $65 \%$ \\
\cline { 2 - 4 } & 8 & $63 \%$ & \\
\hline
\end{tabular}




$-\cdots$ Crustal --- Maule --- Tohoku1 - Tohoku2
- Indonesia $\longrightarrow$ Valparaiso $\cdots$ 84th Perc. Sub.
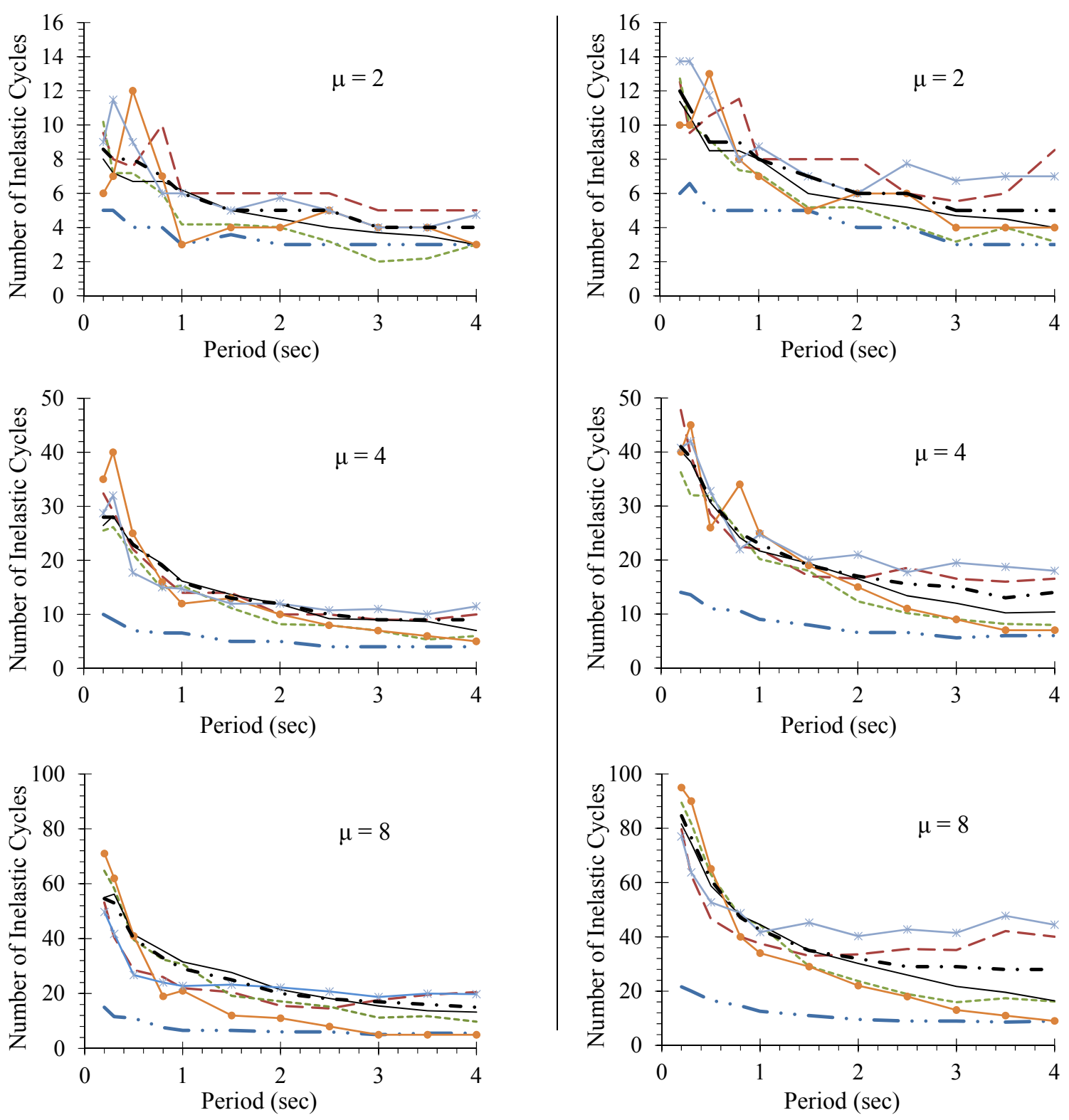

(a)

(b)

Figure 3.4: Number of inelastic cycles for different component ductilities $(\mu)$ :

(a) Pre-Peak excursions; (b) All excursions. 

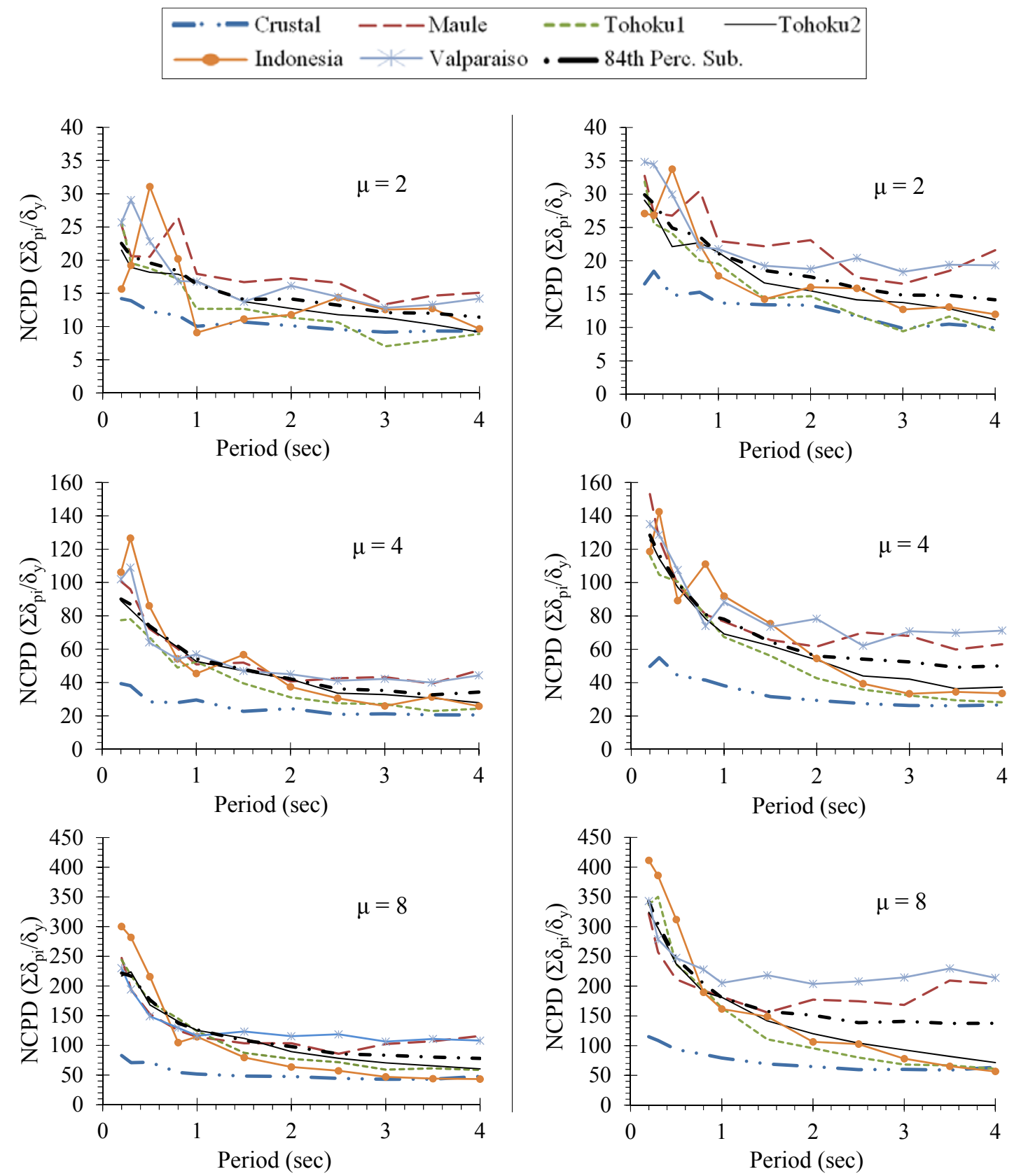

(a)

(b)

Figure 3.5: Normalized Cumulative Plastic Deformation (NCPD) for different component ductilities ( $\mu$ ): (a) Pre-Peak excursions; (b) All excursions. 


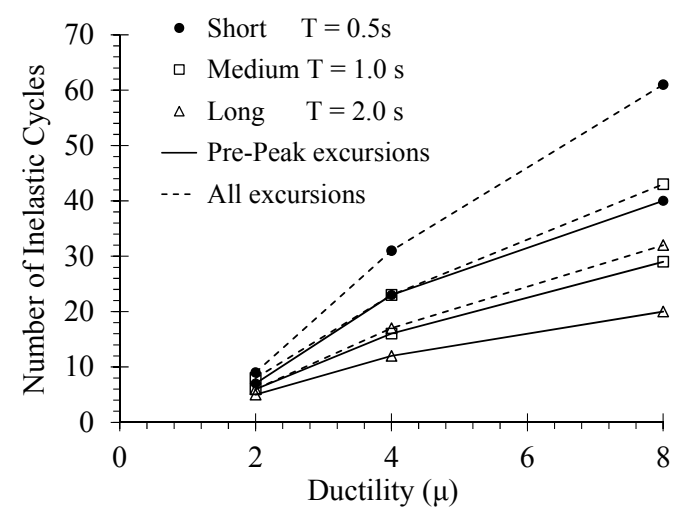

(a)

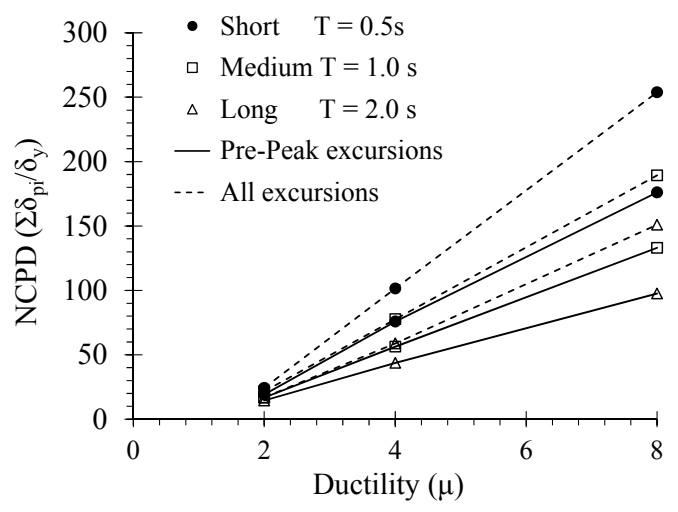

(b)

Figure 3.6: Demand parameter values for different component ductilities.

(a) Number of Inelastic Cycles; (b) Normalized Cumulative Plastic Displacement (NCPD)

\subsection{PROPOSED PROTOCOLS}

The analysis results led to differentiating the testing protocol in terms of ductility and period of the structure. In order to closely reflect the subduction zone demands the loading protocols were developed using the target values of the parameters summarized in Table 3.3 for pre-peak excursions. The proposed loading protocols consider two stages. The first stage consists of three cycles, in each of the following displacements (or loads), $0.25 \delta_{\mathrm{i}}\left(\mathrm{V}_{\mathrm{i}}\right), 0.5 \delta_{\mathrm{i}}\left(\mathrm{V}_{\mathrm{i}}\right), 0.75 \delta_{\mathrm{i}}\left(\mathrm{V}_{\mathrm{i}}\right)$ and one cycle at $1.0 \delta_{\mathrm{i}}\left(\mathrm{V}_{\mathrm{i}}\right)$ in order to visualize low damage states (e.g. first cracking). Where, $\delta_{\mathrm{i}}$ is the theoretical yield displacement and $\mathrm{V}_{\mathrm{i}}$ is the theoretical strength at first yield. The second stage of inelastic cycles aims to replicate the demands imposed on concrete bridge columns by subduction zone earthquakes of long duration. The proposed loading histories, the amplitude of each inelastic cycle and number of cycles at those amplitudes are illustrated in Table 3.4 and Figure 3.7. In Figure 3.7 the dotted lines represent the first stage and the solid lines the second stage. Additional loading protocols considering all the excursions are illustrated in the appendices. 
Table 3.3: Target values and proposed demands considering pre-peak excursions and all excursions

\begin{tabular}{|c|c|c|c|c|c|c|c|c|c|}
\hline \multirow{3}{*}{$\begin{array}{c}\text { Period } \\
\mathbf{T}\end{array}$} & \multirow{3}{*}{$\underset{\mu}{\operatorname{Max}}$} & \multicolumn{4}{|c|}{$\mathbf{N}_{\text {cycles }}>\boldsymbol{\delta}_{\mathrm{y}}$} & \multicolumn{4}{|c|}{ NCPD } \\
\hline & & \multicolumn{2}{|c|}{$\begin{array}{c}\text { Pre-Peak } \\
\text { Excursions }\end{array}$} & \multicolumn{2}{|c|}{ All Excursions } & \multicolumn{2}{|c|}{$\begin{array}{c}\text { Pre-Peak } \\
\text { Excursions }\end{array}$} & \multicolumn{2}{|c|}{ All Excursions } \\
\hline & & $\begin{array}{l}\text { Target } \\
\text { Value }\end{array}$ & $\begin{array}{l}\text { Proposed } \\
\text { Protocol } \\
\end{array}$ & $\begin{array}{l}\text { Target } \\
\text { Value }\end{array}$ & Proposed & $\begin{array}{l}\text { Target } \\
\text { Value } \\
\end{array}$ & $\begin{array}{l}\text { Proposed } \\
\text { Protocol } \\
\end{array}$ & $\begin{array}{l}\text { Target } \\
\text { Value }\end{array}$ & Proposed \\
\hline \multirow{3}{*}{0.5} & 2 & 7 & 9 & 9 & 9 & 19 & 24 & 24 & 24 \\
\hline & 4 & 23 & 23 & 31 & 31 & 76 & 76 & 101 & 103 \\
\hline & 8 & 40 & 40 & 61 & 61 & 176 & 177 & 254 & 253 \\
\hline \multirow{3}{*}{1.0} & 2 & 6 & 9 & 8 & 9 & 17 & 24 & 22 & 24 \\
\hline & 4 & 16 & 16 & 23 & 23 & 56 & 77 & 78 & 77 \\
\hline & 8 & 29 & 29 & 43 & 43 & 133 & 132 & 189 & 188 \\
\hline \multirow{3}{*}{2.0} & 2 & 5 & 6 & 6 & 6 & 14 & 17 & 17 & 17 \\
\hline & 4 & 12 & 12 & 17 & 17 & 44 & 43 & 59 & 59 \\
\hline & 8 & 20 & 20 & 32 & 32 & 98 & 97 & 151 & 151 \\
\hline
\end{tabular}


$\mu=2$
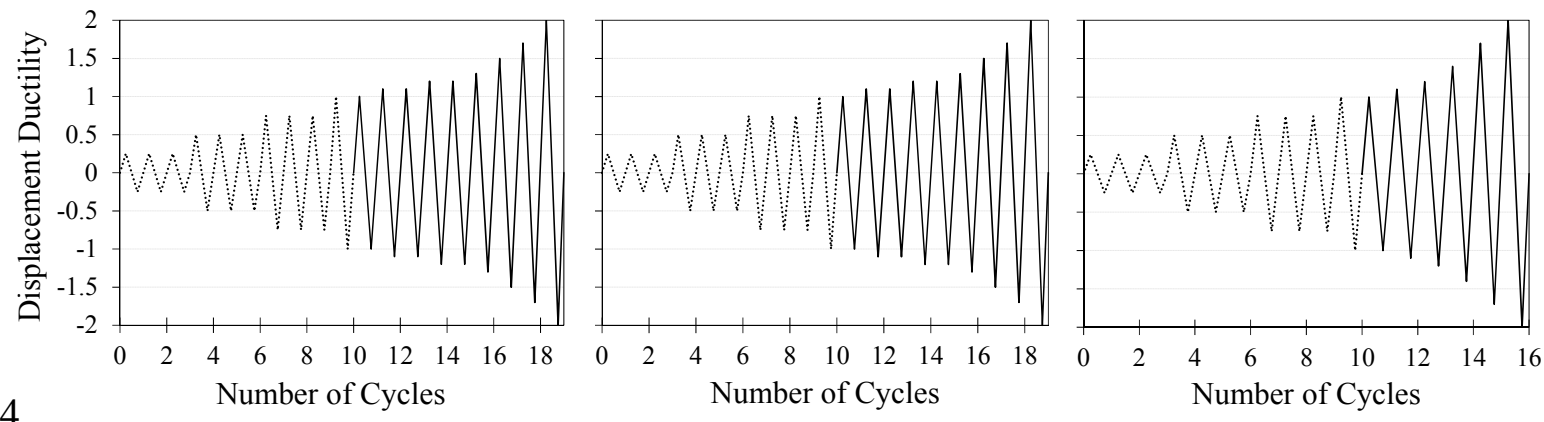

$\mu=4$
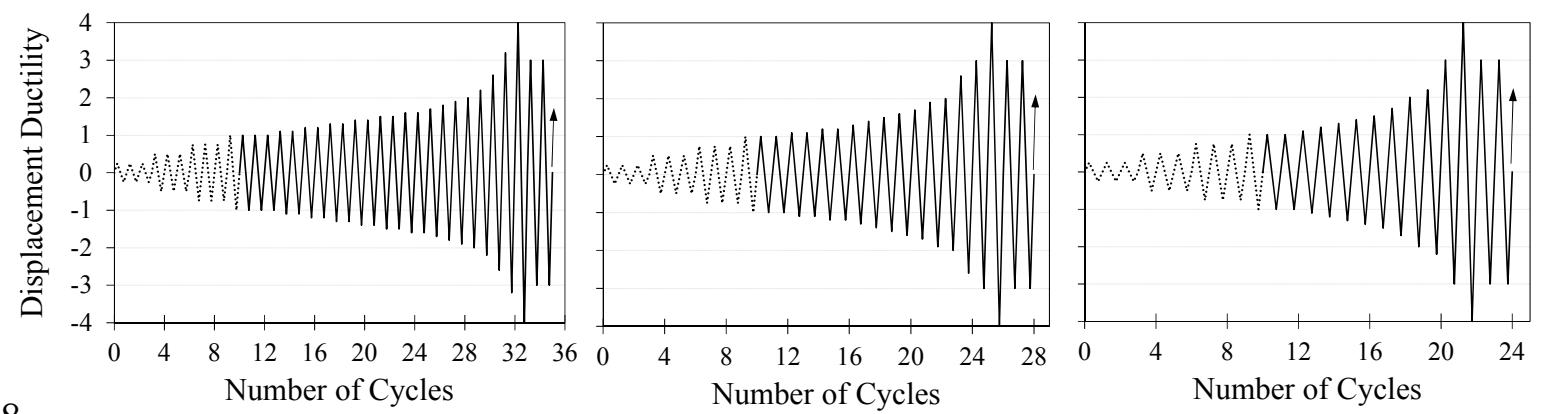

$\mu=8$

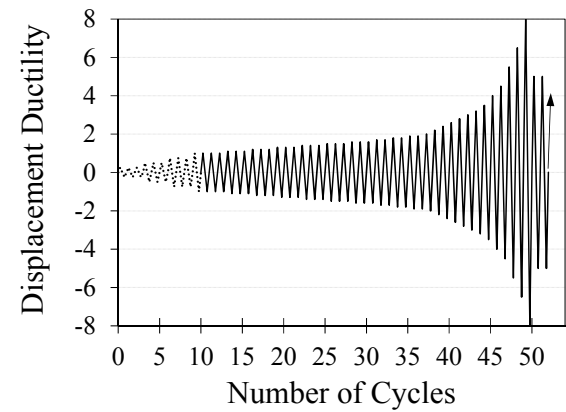

(a)

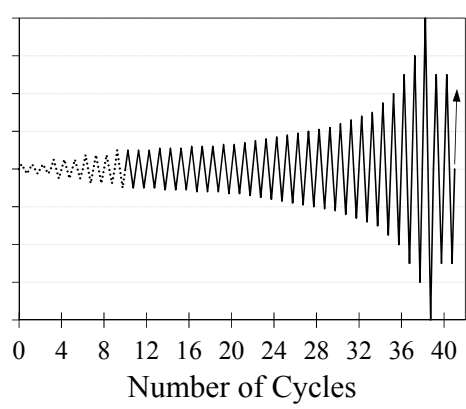

(b)

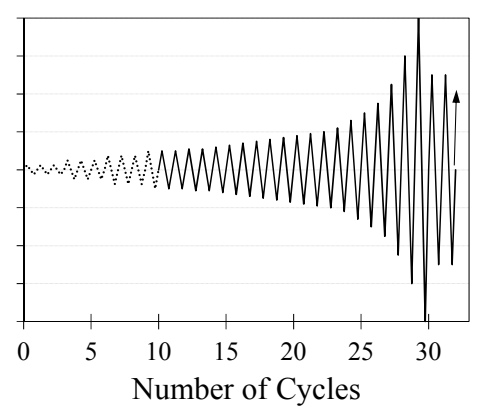

(c)

Figure 3.7: Proposed loading protocols for component ductilities $(\mu)=2,4$ and 8 .

(a) $\mathrm{T}=0.5 \mathrm{sec}$, (b) $\mathrm{T}=1.0 \mathrm{sec}$, (c) $\mathrm{T}=2.0 \mathrm{sec}$. (Pre-peak excursions)

The proposed protocols were developed using the concept of pre-peak excursions. This approach was used since cycles that occur after the maximum displacement will cause less cumulative damage and should be considered separately (Krawinkler et al. 2000a). For that reason, in cases when the specimen does not reach failure under the applied stepwise loading protocol, the test should continue under lower amplitude cycles also referred as trailing cycles. Trailing cycles of amplitude $3 \delta_{\text {yield }}$ for moderate ductile column and $5 \delta_{\text {yield }}$ for highly ductile column were chosen. These values are based on member ductility demand requirements found in AASHTO Seismic Specifications (AASHTO 2009), which state that the maximum individual member displacement ductility demand for Seismic Design Category (SDC) $\mathrm{C}$ shall be equal to 3 and for SDC D shall be equal to 5 for cases of single-column bents. The number of trailing cycles was calculated from the analyses considering all excursions instead of only pre-peak. The number of trailing cycles is shown inside parentheses in Table 3.4. The notation (+ number of trailing cycles) represents the 
number of trailing cycles that are added following the completion of the entire loading protocol. The trailing cycles are at a set amplitude, which is shown in the first column of Table 3.4 and may be either 3 or 5 depending on the proposed protocol to be used.

Table 3.4: Proposed loading protocols

\begin{tabular}{|c|c|c|c|c|c|c|c|c|c|}
\hline \multirow{3}{*}{$\begin{array}{c}\text { Cycle } \\
\text { Amplitude } \\
\mathbf{x} \boldsymbol{\delta}_{\text {yield }}\end{array}$} & \multicolumn{9}{|c|}{ Number of Inelastic Cycles (trailing cycles shown in parentheses) } \\
\hline & \multicolumn{3}{|c|}{ Ductility $(\mu=2)$} & \multicolumn{3}{|c|}{ Ductility $(\mu=4)$} & \multicolumn{3}{|c|}{ Ductility $(\mu=8)$} \\
\hline & $\begin{array}{l}\mathrm{T}= \\
0.5 \mathrm{~s}\end{array}$ & $\begin{array}{l}\mathrm{T}= \\
1.0 \mathrm{~s}\end{array}$ & $\begin{array}{l}\mathrm{T}= \\
2.0 \mathrm{~s}\end{array}$ & $\begin{array}{l}\mathrm{T}= \\
0.5 \mathrm{~s}\end{array}$ & $\begin{array}{l}\mathrm{T}= \\
1.0 \mathrm{~s}\end{array}$ & $\begin{array}{l}\mathrm{T}= \\
2.0 \mathrm{~s}\end{array}$ & $\begin{array}{l}\mathrm{T}= \\
0.5 \mathrm{~s}\end{array}$ & $\begin{array}{l}\mathrm{T}= \\
1.0 \mathrm{~s}\end{array}$ & $\begin{array}{l}\mathrm{T}= \\
2.0 \mathrm{~s}\end{array}$ \\
\hline 1.0 & 1 & 1 & 1 & 3 & 2 & 2 & 3 & 3 & 2 \\
\hline 1.1 & 2 & 2 & 1 & 3 & 2 & 1 & 3 & 3 & 2 \\
\hline 1.2 & 2 & 2 & 1 & 3 & 2 & 1 & 3 & 3 & 1 \\
\hline 1.3 & 1 & 1 & - & 2 & 1 & 1 & 3 & 2 & 1 \\
\hline 1.4 & - & - & 1 & 2 & 1 & 1 & 3 & 1 & 1 \\
\hline 1.5 & 1 & 1 & - & 1 & 1 & 1 & 3 & 1 & 1 \\
\hline 1.6 & - & - & - & 1 & 1 & - & 3 & 1 & 1 \\
\hline 1.7 & 1 & 1 & 1 & 1 & 1 & 1 & 2 & 1 & 1 \\
\hline 1.8 & - & - & - & 1 & - & - & 2 & 1 & 1 \\
\hline 1.9 & - & - & - & 1 & 1 & - & 2 & 1 & 1 \\
\hline 2.0 & 1 & 1 & 1 & 1 & 1 & 1 & 1 & 1 & 1 \\
\hline 2.1 & & & & - & - & - & - & 1 & - \\
\hline 2.2 & & & & 1 & - & 1 & 1 & 1 & 1 \\
\hline 2.4 & & & & - & - & - & 1 & 1 & - \\
\hline 2.6 & & & & 1 & 1 & - & 1 & 1 & 1 \\
\hline 2.8 & & & & - & - & - & 1 & 1 & - \\
\hline 3.0 & & & & $(+5)$ & $1(+4)$ & $1(+3)$ & 1 & 1 & 1 \\
\hline 3.2 & & & & 1 & - & - & 1 & - & - \\
\hline 3.5 & & & & - & - & - & 1 & 1 & 1 \\
\hline 4.0 & & & & 1 & 1 & 1 & 1 & 1 & - \\
\hline 4.5 & & & & & & & 1 & - & 1 \\
\hline 5.0 & & & & & & & $(+8)$ & $1(+6)$ & $(+5)$ \\
\hline 5.5 & & & & & & & 1 & - & - \\
\hline 6.0 & & & & & & & - & 1 & 1 \\
\hline 6.5 & & & & & & & 1 & - & - \\
\hline 8.0 & & & & & & & 1 & 1 & 1 \\
\hline
\end{tabular}

Additionally, the cycle amplitudes of the second stage approximately follow the values obtained using Eq. (3.4) and the appropriate coefficients showed in Table 3.5, which were obtained through regression using an exponential curve on the statistical results obtained from the simplified rainflow counting procedure. For example, in order to obtain the amplitude for the sixth inelastic cycle $(\mathrm{N}=6)$ for a component of ductility 8 and period $0.5 \mathrm{~s}, \mathrm{f}(6)$ that represents the amplitude of the sixth inelastic cycle is equal to 1.06 times the yield displacement $\left(\delta_{\mathrm{y}}\right)$. The maximum number of cycles for each protocol is equal to the respective proposed value shown in 
Table 3.3. For additional comparison, Table 3.5 also shows values considering all excursions in case experimentalists decide those would be more appropriate for their application.

$$
f(N)=\left(a \cdot e^{b \cdot N}+c \cdot e^{d \cdot N}\right) \cdot \delta_{y}
$$

Table 3.5: Exponential coefficients to obtain cycle amplitudes

\begin{tabular}{|c|c|c|c|c|c|c|c|c|c|}
\hline \multirow[b]{2}{*}{ Coeff. } & \multicolumn{3}{|c|}{$\boldsymbol{\mu}=\mathbf{2}$} & \multicolumn{3}{|c|}{$\boldsymbol{\mu}=4$} & \multicolumn{3}{|c|}{$\mu=8$} \\
\hline & $\begin{array}{c}T \\
0.5 \mathrm{~s}\end{array}$ & $\begin{array}{c}T \\
1.0 \mathrm{~s} \\
\end{array}$ & $\begin{array}{c}T \\
2.0 \mathrm{~s}\end{array}$ & $\begin{array}{c}T \\
0.5 \mathrm{~s}\end{array}$ & $\begin{array}{c}T \\
1.0 \mathrm{~s}\end{array}$ & $\begin{array}{c}T \\
2.0 \mathrm{~s}\end{array}$ & $\begin{array}{c}T \\
0.5 \mathrm{~s}\end{array}$ & $\begin{array}{c}T \\
1.0 \mathrm{~s}\end{array}$ & $\begin{array}{c}T \\
2.0 \mathrm{~s}\end{array}$ \\
\hline \multicolumn{10}{|c|}{ Pre-Peak Excursions } \\
\hline $\mathrm{a}$ & 0.9934 & 0.9934 & 0.5215 & 0.9653 & 0.9557 & 0.9638 & 0.8695 & 0.9014 & 0.9869 \\
\hline $\mathrm{b}$ & 0.0221 & 0.0221 & $\begin{array}{c}- \\
0.3778 \\
\end{array}$ & 0.0281 & 0.0439 & 0.0543 & 0.0336 & 0.0415 & 0.0444 \\
\hline $\mathrm{c}$ & 0.0137 & 0.0137 & 0.5674 & $\begin{array}{c}1.15 \mathrm{E}- \\
4\end{array}$ & $\begin{array}{c}8.91 \mathrm{E}- \\
4\end{array}$ & $\begin{array}{c}2.72 \mathrm{E}- \\
3\end{array}$ & $\begin{array}{c}1.913 \mathrm{E}- \\
7\end{array}$ & $\begin{array}{c}2.83 \mathrm{E}- \\
5\end{array}$ & $\begin{array}{c}1.09 \mathrm{E}- \\
3\end{array}$ \\
\hline $\mathrm{d}$ & 0.4511 & 0.4511 & 0.2066 & 0.4284 & 0.4853 & 0.5570 & 0.4252 & 0.417 & 0.4282 \\
\hline \multicolumn{10}{|c|}{ All Excursions } \\
\hline $\mathrm{a}$ & \multirow{4}{*}{\multicolumn{3}{|c|}{$\begin{array}{c}\text { Same as Pre-Peak } \\
\text { Excursions }\end{array}$}} & 0.9505 & 0.9335 & 0.936 & 0.8356 & 0.8659 & 0.8931 \\
\hline $\mathrm{b}$ & & & & 0.0245 & 0.0357 & 0.0485 & 0.0224 & 0.0314 & 0.0433 \\
\hline $\mathrm{c}$ & & & & $\begin{array}{c}1.18 \mathrm{E}- \\
5 \\
\end{array}$ & $\begin{array}{c}3.14 \mathrm{E}- \\
5 \\
\end{array}$ & $\begin{array}{c}5.57 \mathrm{E}- \\
5 \\
\end{array}$ & $1.84 \mathrm{E}-8$ & $\begin{array}{c}3.45 \mathrm{E}- \\
7 \\
\end{array}$ & $3.4 \mathrm{E}-6$ \\
\hline $\mathrm{d}$ & & & & 0.3875 & 0.478 & 0.613 & 0.3167 & 0.3816 & 0.4398 \\
\hline
\end{tabular}

Since the proposed protocols are based on increments of ductility demand, determining the yield displacement of the specimen is essential and mirrors other cyclic protocols. To overcome this, a first estimate of the yield displacement shall be found by performing a moment-curvature analysis of the column section based on measured material properties. Experimentalists can modify the testing protocol for structures with different ductility in order to represent the target ductility demand appropriate for their desired application, in which case interpolation of the demand values presented in Table 3.3 is recommended.

In order to determine the yield displacement $(\delta y)$ to be used in an experimental test researchers have employed two approaches. The first consists of performing a monotonic pushover test before cyclic loading tests. This approach is used for three main reasons. First of all, the yield displacement for future cyclic tests can be established based on the monotonic test. Secondly, it reflects that the structural response under seismic excitations usually shows an increase of deformations in only one direction, phenomenon denominated "ratcheting" of the response, which implies that cyclic deterioration under load reversals would diminish in the inelastic range close to the system collapse (Lignos and Krawinkler 2012). Finally, a monotonic test would provide the value of target ductility in cases when more refined cyclic loading protocols want to be performed. The second approach consists of determining the yield displacement during the 
progression of the cyclic test. Load control cycles are first employed based on percentages of the theoretical component strength (Vi), usually $0.25 \mathrm{Vi}, 0.5 \mathrm{Vi}, 0.75 \mathrm{Vi}$, and $\mathrm{Vi}$. The theoretical strength is determined dividing the first yield moment, obtained from a moment-curvature analysis, by the column cantilever length. Then the experimental yield displacement ( $\delta y)$ is established by using the ratio of the theoretical force at which the concrete cover reaches a strain of 0.004 to the experimental elastic stiffness $(\mathrm{Ke})$, which is calculated as the ratio of the theoretical first yield force $(\mathrm{Vi})$ to the displacement measured experimentally ( $\left.\delta y^{\prime}\right)$ as illustrated in Figure 3.8 (Priestley et al. 2002).

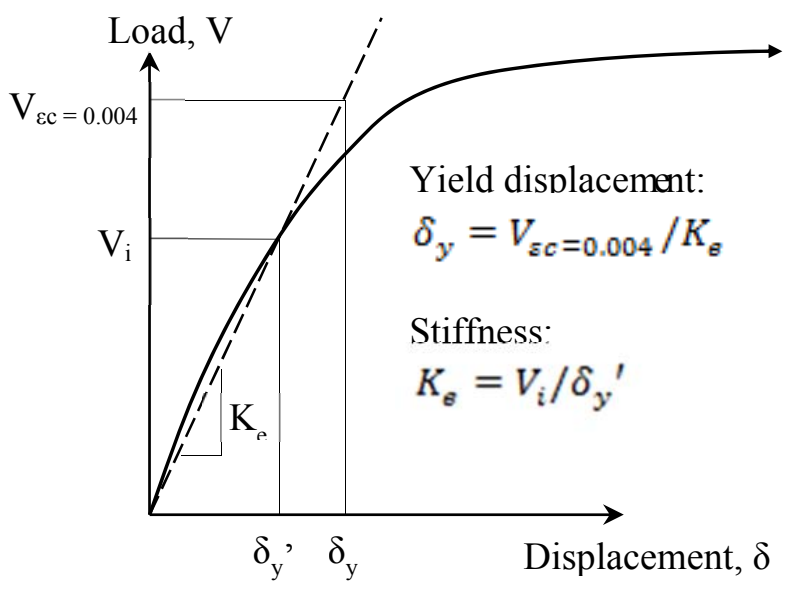

Figure 3.8: Determination of yield displacement

\subsection{COMPARISON TO CONVENTIONAL PROTOCOLS}

In order to assess the seismic performance of bridges, it is common that reinforced concrete members are tested using conventional cyclic loading protocols as those shown in Figure 3.1 (Cheung et al. 1991) (Priestley et al. 2002), (Kowalsky et al. 2009). Experimental studies (Kunnath et al. 1997), (McDaniel et al. 2006), (Song and Lee 2012) have demonstrated that these type of loading protocols may not be representative of the demands imposed by ground motion excitations, which would generally subject the structure to numerous small inelastic cycles and only few large inelastic cycles before collapse occurs.

An illustrative comparison of three cyclic protocols commonly used to assess the capacity of reinforced concrete bridge columns with the proposed protocol for a target ductility capacity of 8 and period $0.5 \mathrm{sec}$ is summarized in Figure 3.9. Since the different protocols culminate in the same ductility demand, they were ordered to end at the same cycle to allow the reader a more clear visual comparison. This led to clearly differentiate among protocols in terms of the number of inelastic cycles and their amplitude. The three selected conventional protocols were the New Zealand protocol (Cheung et al. 1991), the Modified New Zealand Protocol (Priestley et al. 2002), and the ACI374 protocol (ACI 374.2R-13 2013).

Computed demand parameters for the three conventional protocols and the proposed are also compared in Table 3.6. The modified N.Z. protocol is observed to be more demanding in terms of NCPD than the proposed testing protocols for structures of low ductility capacity in the range 
of periods selected, and for structures of high ductility capacity with medium and long fundamental periods. The fact that the modified N.Z protocol is more demanding than the proposed protocols for structures with medium and long fundamental periods was not necessarily surprising since conventional protocols have been developed to reflect the demands in short period structures $(\mathrm{T}=0.2-0.3 \mathrm{sec})$ and because their arrangement of cycles tends to overestimate the number of large inelastic cycles imposed by seismic excitations (FEMA 461 2007), (ACI $374.2 R-13$ 2013). This overestimation is evident in that over $55 \%$ of the cumulative demand (NCPD) using the conventional protocols came from cycles of large amplitude, as compared to $16 \%$ in the case of the proposed protocols for short period structures with high ductility capacity, and $44 \%$ in the case of long period structures with low ductility capacity.

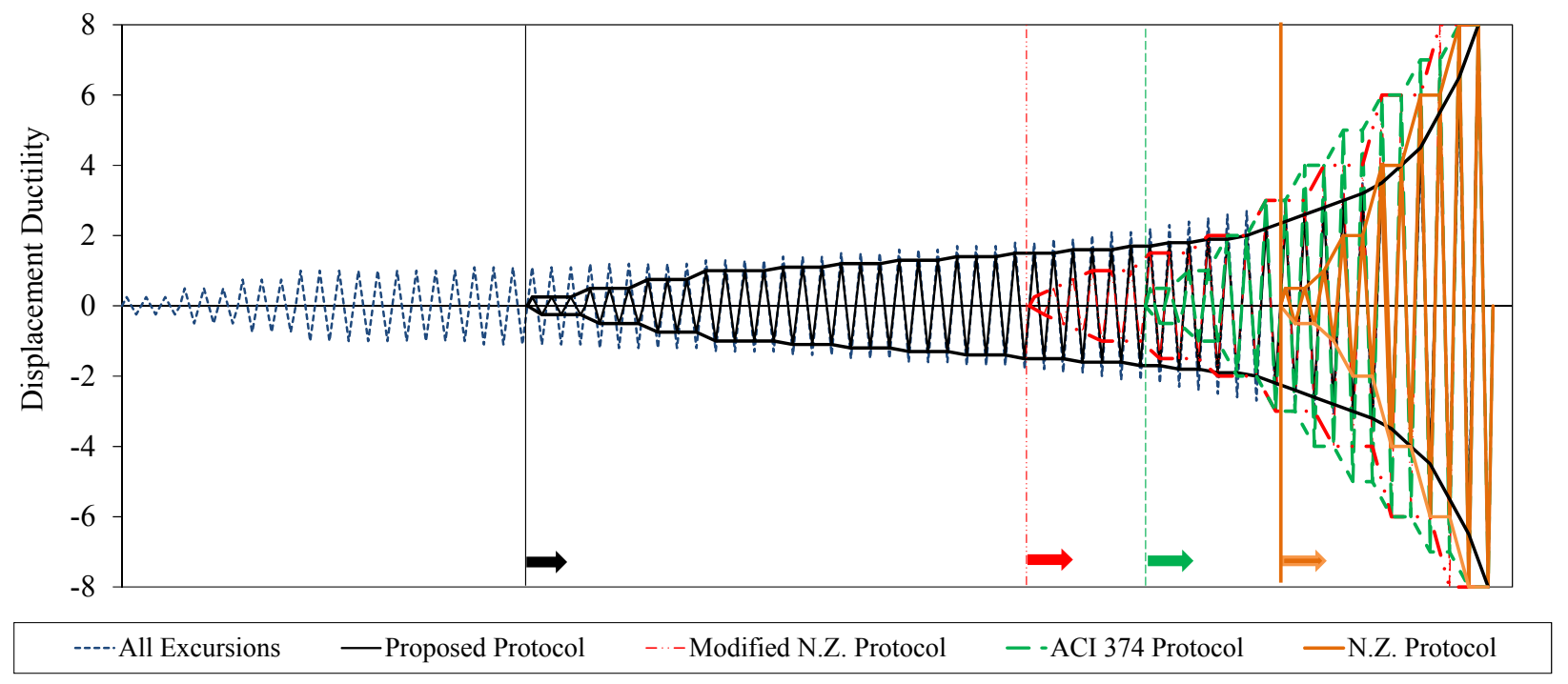

Figure 3.9: Illustrative comparison of protocols for bridge columns of ductility capacity 8 and period $0.5 \mathrm{sec}$.

Table 3.6: Comparison of conventional protocols and proposed protocols

\begin{tabular}{|c|c|c|c|c|c|c|}
\hline \multirow[b]{2}{*}{ Demand Parameter } & \multirow[b]{2}{*}{$\begin{array}{c}\text { N.Z. } \\
\text { Protocol }\end{array}$} & \multirow[b]{2}{*}{$\begin{array}{l}\text { ACI } 374 \\
\text { Protocol }\end{array}$} & \multirow{2}{*}{$\begin{array}{c}\text { Modified } \\
\text { N.Z. } \\
\text { Protocol }\end{array}$} & \multicolumn{3}{|c|}{ Proposed Protocols } \\
\hline & & & & $\begin{array}{c}\mathrm{T}=0.5 \\
\mathrm{~s}\end{array}$ & $\begin{array}{c}\mathrm{T}=1.0 \\
\mathrm{~S}\end{array}$ & $\mathrm{~T}=2.0 \mathrm{~s}$ \\
\hline \multicolumn{7}{|c|}{ Ductility $(\mu)=2$} \\
\hline No. Inelastic Cycles & 3 & 4 & 9 & 9 & 9 & 6 \\
\hline NCPD & 10 & 12 & 27 & 24 & 24 & 17 \\
\hline$(\mathrm{CPD} \geq 1.5 \quad \mathrm{y}) / \mathrm{NCPD}^{1}$ & $80 \%$ & $67 \%$ & $78 \%$ & $43 \%$ & $43 \%$ & $44 \%$ \\
\hline \multicolumn{7}{|c|}{ Ductility $(\mu)=4$} \\
\hline No. Inelastic Cycles & 5 & 8 & 15 & 23 & 16 & 12 \\
\hline NCPD & 26 & 40 & 69 & 76 & 56 & 43 \\
\hline$(\mathrm{CPD} \geq 3 \quad \mathrm{y}) / \mathrm{NCPD}$ & $62 \%$ & $70 \%$ & $61 \%$ & $19 \%$ & $25 \%$ & $33 \%$ \\
\hline \multicolumn{7}{|c|}{ Ductility $(\mu)=8$} \\
\hline No. Inelastic Cycles & 9 & 16 & 21 & 40 & 29 & 20 \\
\hline NCPD & 82 & 144 & 153 & 177 & 132 & 97 \\
\hline$(\mathrm{CPD} \geq 6 \quad \mathrm{y}) / \mathrm{NCPD}$ & $68 \%$ & $58 \%$ & $55 \%$ & $16 \%$ & $21 \%$ & $29 \%$ \\
\hline
\end{tabular}

Percentage of cumulative normalized plastic displacement due to large inelastic cycles 
A visual comparison is shown in Figure 3.10 for the cumulative displacement ductility demand among the proposed protocols for period $0.5 \mathrm{sec}$, the conventional protocols and the results from the statistical analyses using the $84^{\text {th }}$ percentile of the demand parameters as target value. Proposed protocols closely resemble the cumulative demand obtained by using the statistical target values, which in Figure 3.10 are referred to as analysis results. This correlation verifies the appropriate selection of the discrete amplitudes for the proposed protocols relative to the finer resolution statistical target values. A period of $0.5 \mathrm{sec}$ was selected as the closest period to make comparisons since the conventional protocols were developed for structures of period equal to 0.2 seconds ( $A C I 374.2 R-13$ 2013). Longer periods may not be representative and could lead to incompatible comparisons. Despite the differences in short period definition, the proposed protocols considering the subduction megathrust earthquakes exhibit higher cumulative ductility demand than the conventional protocols. This difference could be even larger had the proposed protocol considered even shorter period than $0.5 \mathrm{sec}$ structural response.
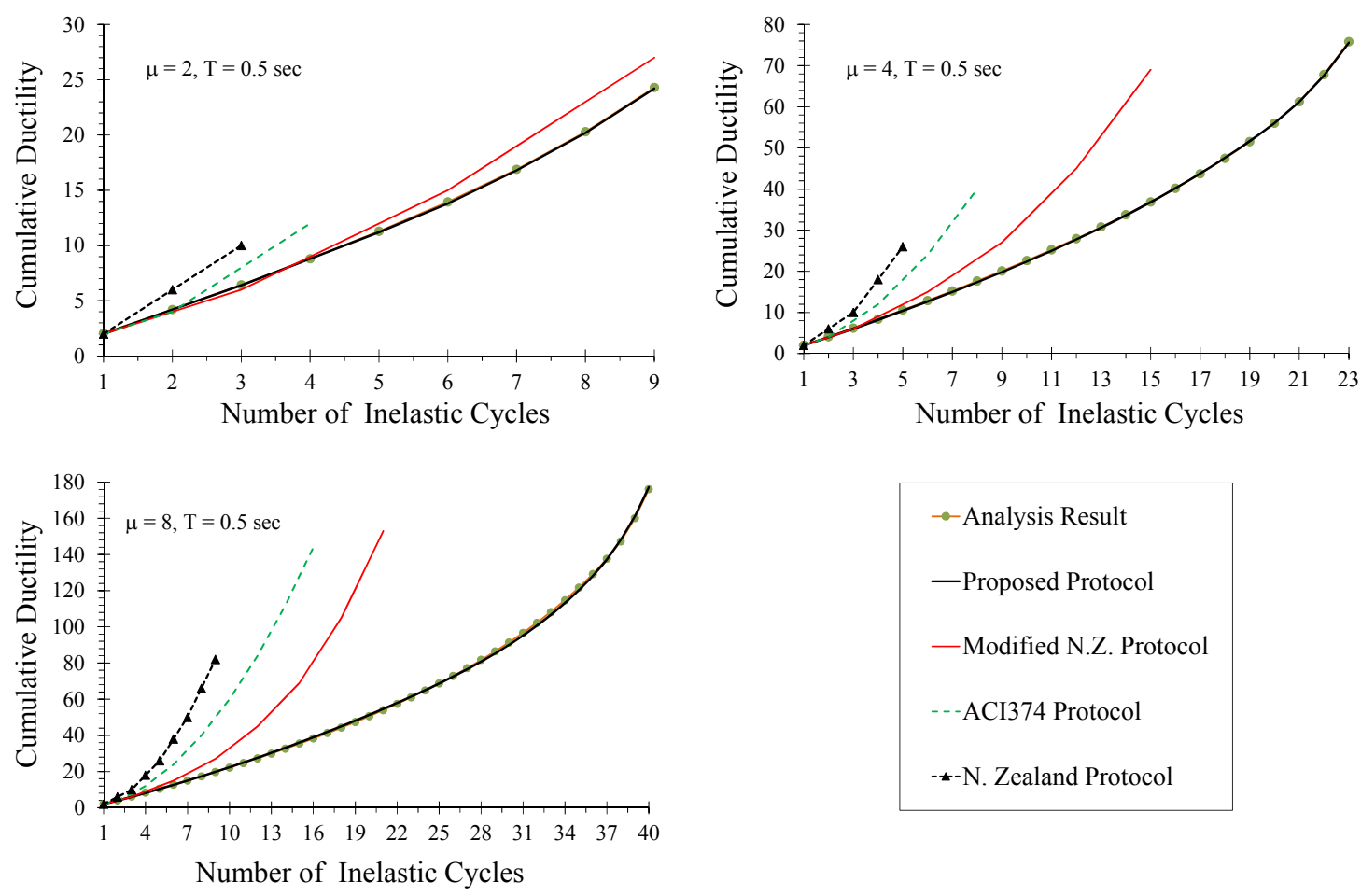

- Analysis Result

- Proposed Protocol

—Modified N.Z. Protocol

---ACI374 Protocol

$-\star-N$. Zealand Protocol

Figure 3.10: Comparison of cumulative ductility demands for component ductilities 2, 4 and 8 and component fundamental period of $0.5 \mathrm{sec}$.

In Figure 3.11 the cumulative distribution function (CDF) of the proposed protocols for structures of period $0.5 \mathrm{sec}$ is compared with the loading protocols commonly used on RC columns and the results from the statistical analyses. The conventional protocols used for the assessment of RC columns contain far fewer inelastic cycles than the proposed protocol. Despite the overestimation of the number of large inelastic cycles in the conventional protocols, the 
proposed protocols for structures of moderate and high ductility capacity exhibit higher demands than the conventional protocols due to a substantial increase in the number of small inelastic cycles. This difference is mainly due to the fact that the proposed protocol tries to reflect the demands imposed by subduction earthquake excitations, which generally contain many small inelastic cycles and only a few large inelastic cycles.

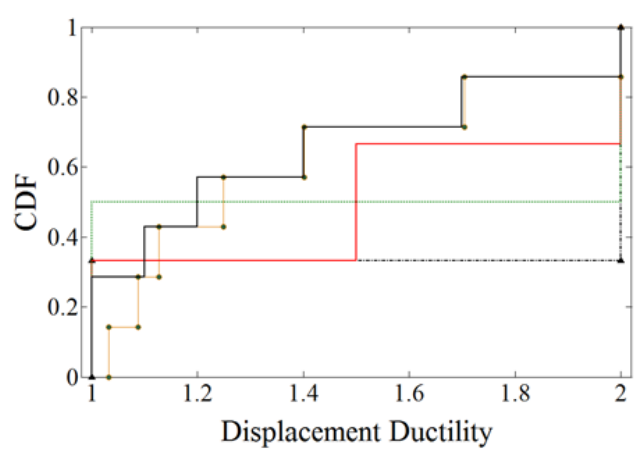

(a)

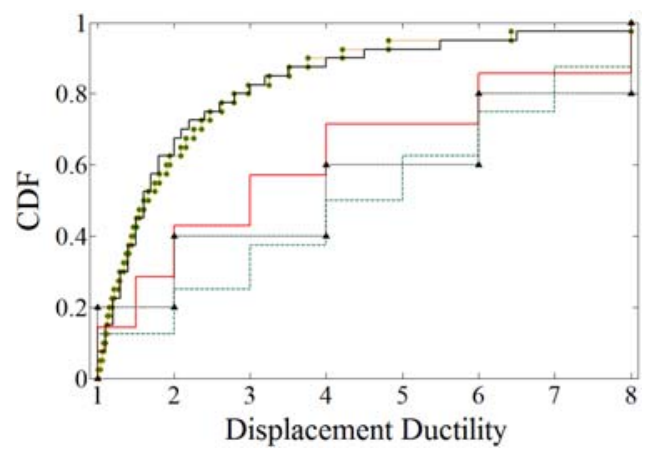

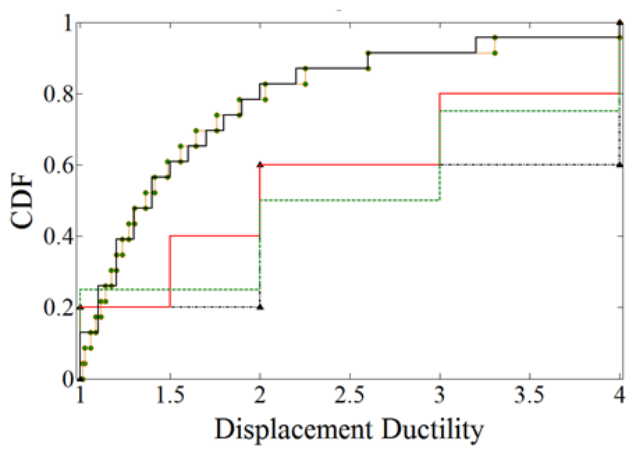

(b)

(c)

Figure 3.11: Comparison of cumulative distribution functions for conventional protocols, proposed protocol, and statistical result for structures of period $0.5 \mathrm{sec}$ and different component ductilities. (a) $\mu=2$, (b) $\mu=4$, (c) $\mu=8$.

\subsection{SUMMARY}

Observations based on the development of cyclic loading protocols can be summarized as follows:

- The assessment of bridge columns through representative cyclic protocols could play a significant role in the future establishment of limit states and acceptance criteria to be applied in performance-based seismic design of bridge columns in areas subjected to subduction earthquake hazard.

- The simplified rainflow procedure was employed to convert the inelastic response obtained from extensive non-linear time history analyses utilizing recorded strong motion data from subduction megathrust earthquakes into symmetric cycles. This 
procedure also allowed for computation of demand parameters such as the number of inelastic cycles and the normalized cumulative plastic displacement metric.

Observations based on these parameters showed that for structural periods below $2.0 \mathrm{~s}$, the subduction mega earthquakes produced significantly greater number of inelastic cycles as well as cumulative plastic displacements than crustal earthquakes. Statistical assessment of the demand parameters were used to develop quasi-static loading protocols. Due to the dependence of the results on structural ductility and natural period of vibration, different loading protocols were proposed for three column ductilities $(2,4$ and 8$)$ and for three different periods representing short, medium and long fundamental periods.

- The proposed loading protocols include a larger number of small amplitude inelastic cycles as compared to conventional protocols, revealing that conventional loading protocols commonly used in experimental testing tend to impose unrepresentative drift demands for subduction megathrust earthquakes through imposing numerous large inelastic reversals on the component. Despite the higher number of large inelastic cycles, the overall normalized cumulative plastic displacement demands were similar when compared to the proposed protocols. The proposed subduction protocols may influence the response of reinforced concrete columns due to an increase in the overall number of inelastic cycles and should be considered when megathrust subduction earthquake hazard affects the design criteria. 


\subsection{ASSESSMENT OF SEISMICALLY DEFICIENT RC BRIDGE COLUMNS}

\subsection{GENERAL}

In this chapter the influence of one of the proposed protocols on a representative deficient bridge column built before 1970 in the State of Oregon is analytically and experimentally examined.

As discussed in previous chapters, the cumulative inelastic demands imposed by subduction zone earthquakes can increase as compared to crustal earthquake demands. This aspect is particularly relevant in the State of Oregon and the Northwest coast of North America due to their proximity to the Cascadia subduction zone. The Cascadia subduction zone is the over $1000 \mathrm{~km}$ long boundary between the Juan de Fuca and North American plates. Geological evidence has shown that 13 significant earthquakes have occurred in the past 3000 years (Goldfinger et al. 2008). The most notable of which, the M9.0 earthquake of 1700, produced a tsunami large enough to reach Japan (Atwater et al. 2005). Historical evidence combined with comparisons of the Cascadia fault to other subduction zones has led geologists to conclude that a megathrust earthquake in the Cascadia subduction zone is impending (Heaton and Kanamori 1984). Given such seismic hazard, the assessment of deficient RC bridge substructures has become crucial in order to prioritize the bridges that need to be retrofitted and to maintain the highway network operable after a major seismic event.

\subsection{REPRESENTATIVE RC BRIDGE COLUMN}

In the 2010 ODOT inventory, there are over 9,864 bridges and culverts. The state highway agency owns 5,280 of these. However, only 2,567 of the highway bridges are in the NBI database of which 1,997 are multi span. Figure 4.1 shows the bridge classes and proportions of these multi span bridges. This section is focused on multi-span continuous stringer/girder RC bridges that were built before 1970 because are the most common type of bridge in Oregon.

Reinforced concrete columns constructed before 1970 are commonly deficient in flexural ductility and shear strength as bridges were designed primarily for gravity loads without much consideration to lateral forces from seismic loading as illustrated in Figure 4.2. As a result, columns lack sufficient transverse reinforcement to provide satisfactory performance in a major seismic event. Typically, No. 3 or No. 4 hoops at 12 inches on center were provided in columns regardless of the column cross-sectional dimensions. The stirrups were anchored by $90^{\circ}$ hooks with short extensions and intermediate ties were seldom used. Minimal restraint provided by the hoops can cause the longitudinal reinforcement to buckle once the concrete cover spalls. Furthermore, bridges built prior to 1970 have undesirable lap splices at the base of RC column. This lap splice detail can potentially be a cause for reduced column ductility and can result in rapid loss of flexural strength. Typical details for the representative multi-span continuous stringer/girder RC bridge is illustrated in Figure 4.3. 


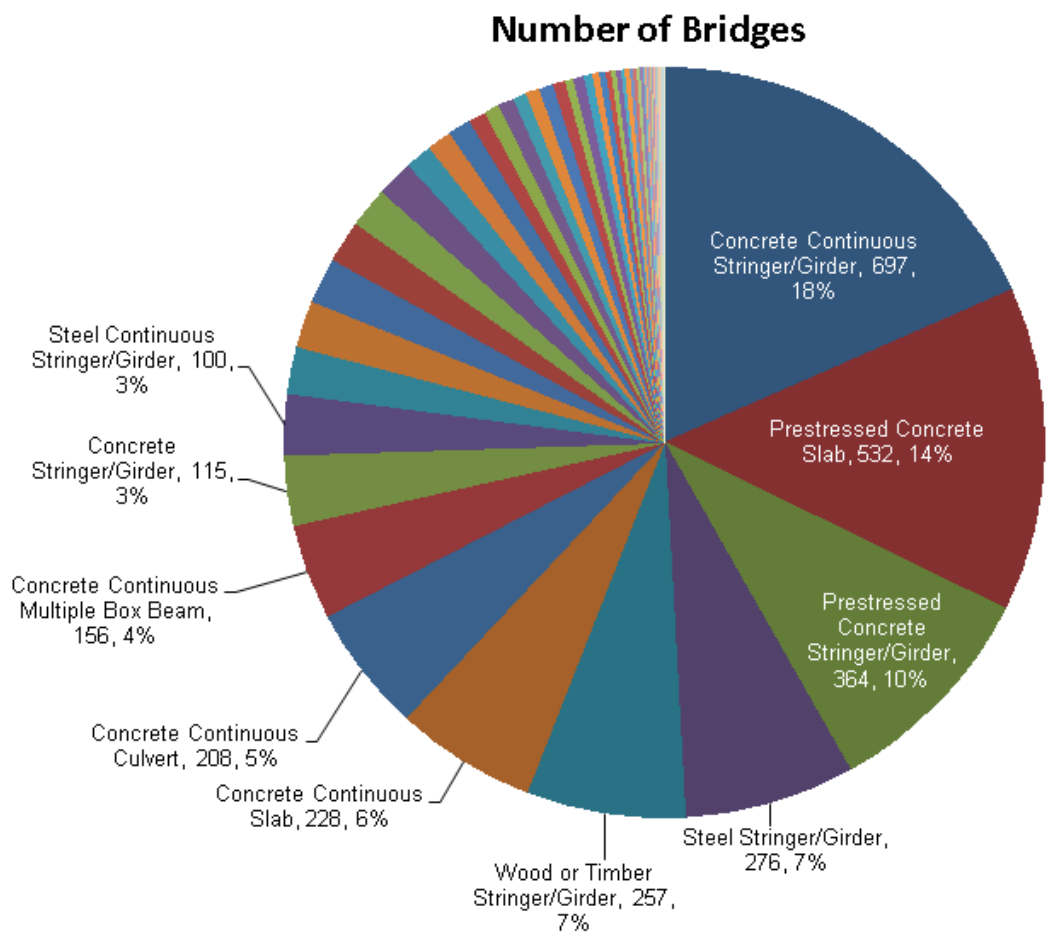

Figure 4.1: Bridge Classes

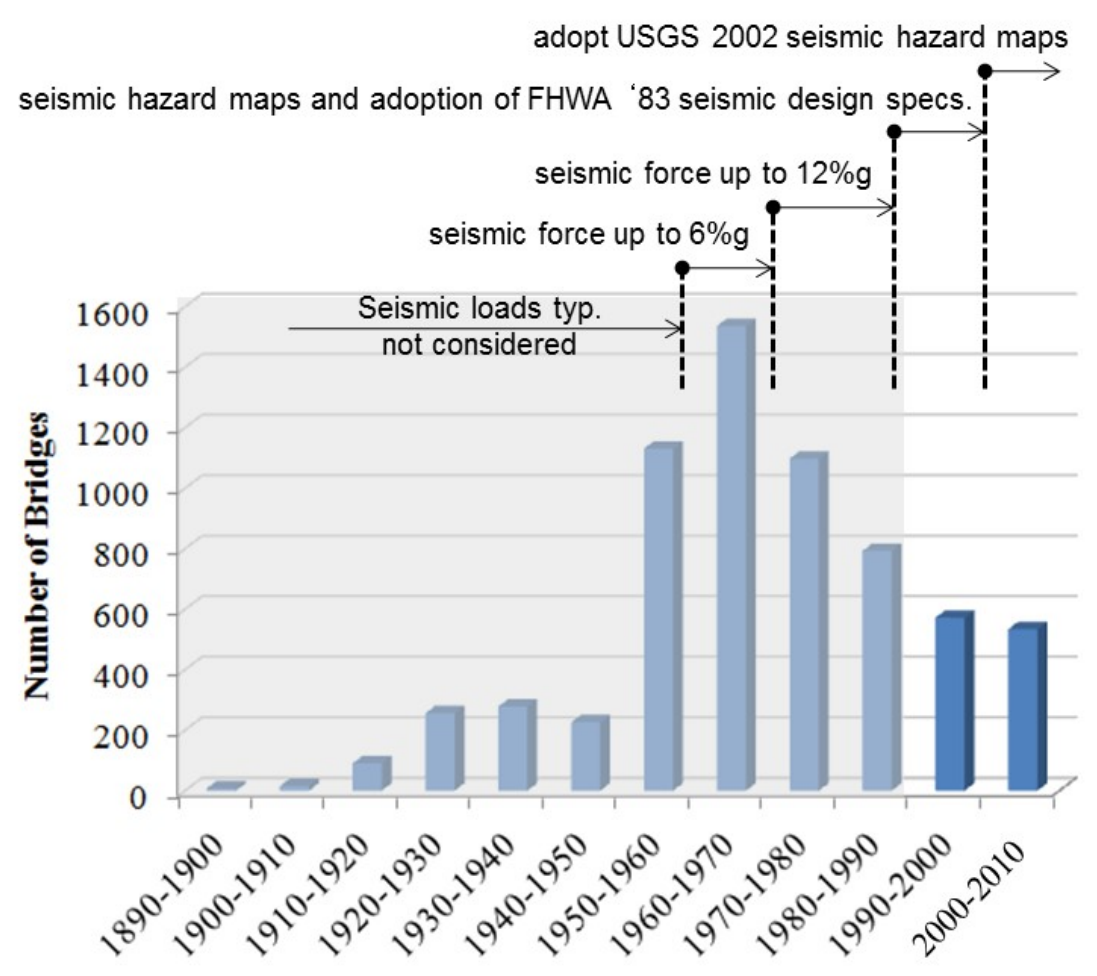

Figure 4.2: Illustration of seismic loading consideration over the years. 


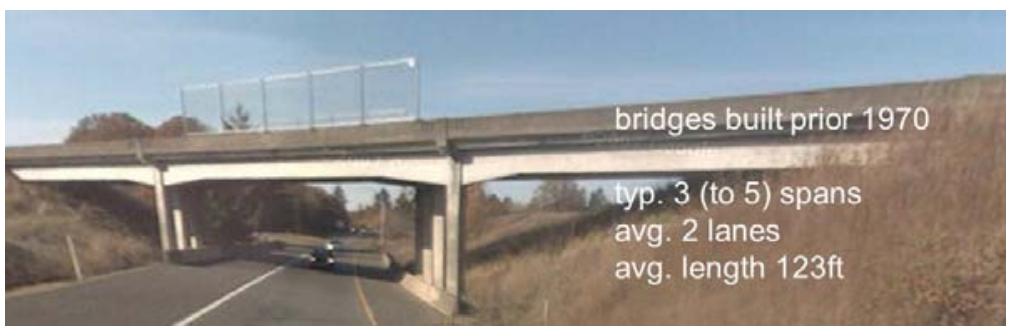

(a)

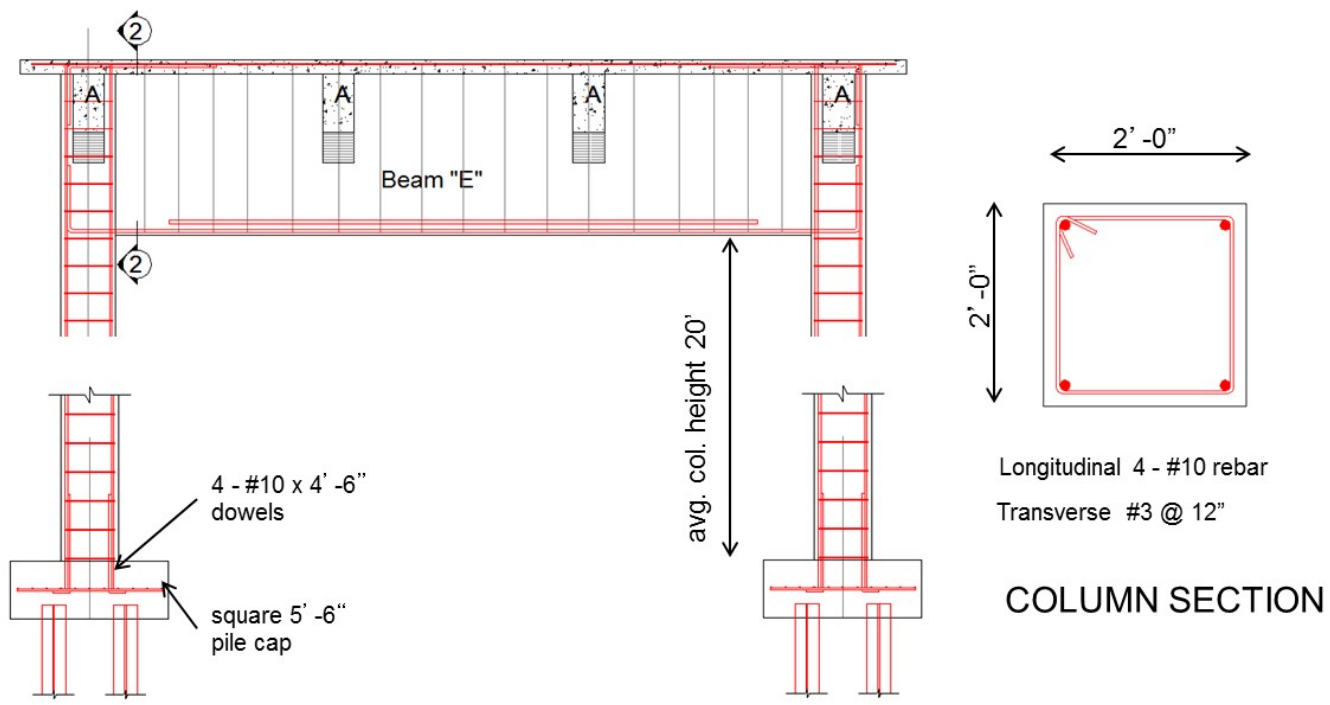

(b)

Figure 4.3: Typical multi-span continuous stringer/girder RC bridge. (a) Picture. (b) Reinforcement details.

\subsection{NUMERICAL CASE STUDY}

The illustrative numerical case study presented in this section aims to assess the behavior of pre1970 bridge columns located in the State of Oregon, USA. The case study contemplates the numerical study of a representative pre-1970 bridge column subjected to a conventional protocol and a subduction protocol. The modified New Zealand protocol was used as the conventional protocol and the proposed protocol for structures of ductility 8 and fundamental period of $0.5 \mathrm{sec}$ was used as the subduction protocol. Protocols with that target ductility were used because the ductility obtained from moment-curvature analysis of the column in study was close to 8. Both protocols used in the numerical case study are shown in Chapter 3.

Typical RC columns built before 1970 in the State of Oregon are usually lightly reinforced and lap-spliced in places where plastic hinge formation is expected. Typical column properties and dimensions are summarized in Table 4.1 and the cross section is illustrated in Figure 4.3. 
Table 4.1: Column properties and dimensions.

\begin{tabular}{c|c|c|c|c|c|c|c|c|c|c|c}
\hline $\begin{array}{c}\mathrm{f}_{\mathrm{c}} \\
(\mathrm{ksi})\end{array}$ & $\begin{array}{c}\mathrm{f}_{\mathrm{ce}}^{\prime} \\
(\mathrm{ksi})\end{array}$ & $\begin{array}{c}\mathrm{f}_{\mathrm{y}} \\
(\mathrm{ksi})\end{array}$ & $\begin{array}{c}\mathrm{f}_{\mathrm{ye}} \\
(\mathrm{ksi})\end{array}$ & $\begin{array}{c}\text { Cantilever } \\
\text { Length } \\
(\mathrm{ft})\end{array}$ & $\begin{array}{c}\text { Width } \\
(\mathrm{in})\end{array}$ & $\begin{array}{c}\text { Depth } \\
(\mathrm{in})\end{array}$ & $\begin{array}{c}\text { Axial } \\
\text { Load } \\
(\mathrm{kip})\end{array}$ & $\begin{array}{c}\text { Axial } \\
\text { Load } \\
\text { Ratio }{ }^{1}(\%)\end{array}$ & $\begin{array}{c}\rho_{\mathrm{T}} \\
(\%)\end{array}$ & $\begin{array}{c}\rho_{\mathrm{L}} \\
(\%)\end{array}$ & $\begin{array}{c}\text { Lap Splice } \\
\text { Length } \\
\left(\mathrm{d}_{\mathrm{b}}\right)\end{array}$ \\
\hline 3.3 & 4.29 & 60 & 68 & 9.25 & 24 & 24 & 160 & 8.4 & 0.094 & 0.88 & 28 \\
\hline
\end{tabular}

${ }^{2}$ Axial load ratio $=\mathrm{P} /\left(\mathrm{A}_{\mathrm{g}} \mathrm{f}_{\mathrm{c}}{ }_{\mathrm{c}}\right)$

Where,

$\mathrm{f}^{\prime}$ : : specified compressive strength of concrete at 28 days.

$\mathrm{f}^{\prime}{ }_{\mathrm{ce}}$ : expected compressive strength of concrete. $\mathrm{f}^{\prime}{ }_{\mathrm{ce}}=1.3\left(\mathrm{f}_{\mathrm{c}}{ }_{\mathrm{c}}\right)$

$f_{y}$ : specified yield stress of steel.

$\mathrm{f}_{\mathrm{ye}}$ : expected yield stress of steel. $\mathrm{f}_{\mathrm{ye}} \approx 1.1 \mathrm{f}_{\mathrm{y}}$

$\rho_{\mathrm{T}}$ : transverse ratio of steel.

$\rho_{\mathrm{L}}$ : Longitudinal ratio of steel.

$\mathrm{d}_{\mathrm{b}}$ : diameter of longitudinal steel reinforcement.

In order to model the inelastic behavior of the column the concentrated plasticity approach was utilized. The plastic hinge was modeled using the hysteretic model developed by Ibarra et al. (Ibarra et al. 2005a), as illustrated in Figure 4.4, and implemented in the software OpenSees (OpenSees 2011). Model parameters for column hinges, such as moment capacity and rotation capacity, have been obtained from empirical equations based on a vast amount of column tests (Haselton et al. 2008) (Biskinis and Fardis 2009).

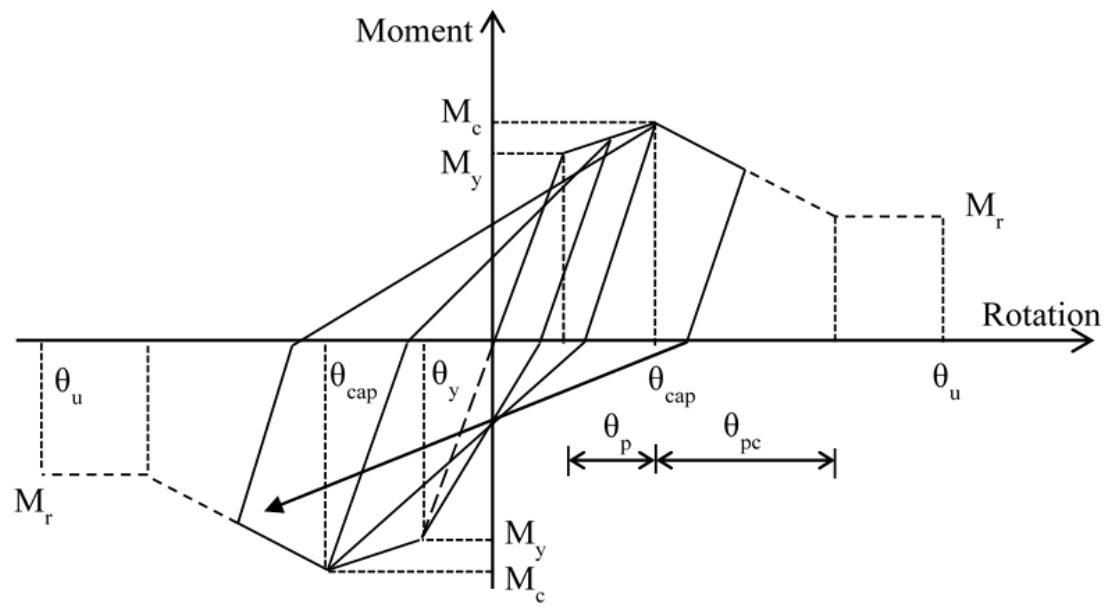

Figure 4.4: Hysteretic behavior used for the numerical case study. Adapted from Ibarra et al. (Ibarra et al. 2005a) 
The hysteretic energy dissipation capacity plays a fundamental role in the assessment of bridge columns subjected to subduction zone ground motion. Haselton et al. (Haselton et al. 2008) has proposed equations to calculate this capacity $(\lambda)$, which according his equation depends on the amount of transverse reinforcement, shear capacity and axial load ratio. Another equation also proposed by Haselton is included in the PEER/ATC 72-1 (PEER/ATC 71-1 2010) report, in which the value of $\lambda$ only depends on the axial load ratio. The PEER/ATC report stated that for a typical column with seismic detailing, typical values of the parameter $\lambda$ are on the order of 10 to 20. On the other hand, in the study carry out by Haselton (Haselton 2008) values from 2 to 5 were employed for highly deteriorated components. This means that a lower $\lambda$ indicates that the element has a high rate of strength and stiffness deterioration and therefore less capacity to dissipate energy. Since pre-1970 columns were built without seismic detailing the behavior of these columns is expected to be represented by $\lambda$ values near 2 .

The model parameters using equations proposed by Haselton (Haselton 2008), Biskinis (Biskinis 2009), and moment-curvature analysis are summarized in Table 4.2. The moment -curvature analysis was based on conventional reinforced concrete flexure theory following AASHTO Specifications (AASHTO 2009) and is shown in the Appendices. It is worth mentioning that all the analyses utilized the expected material properties, where $\mathrm{f}^{\prime}{ }_{\mathrm{ce}}=1.3 \mathrm{f}^{\prime}{ }_{\mathrm{c}}$ and $\mathrm{f}_{\mathrm{ye}} \approx 1.1 \mathrm{f}_{\mathrm{y}}$.

Table 4.2: Model Parameters

\begin{tabular}{c|c|c|c|c|c|c|c|c|c}
\hline Reference & $\begin{array}{c}\mathbf{M y} \\
(\mathbf{k N}- \\
\mathbf{m})\end{array}$ & $\mathbf{M}_{\mathbf{c}} / \mathbf{M}_{\mathbf{y}}$ & $\mathbf{E I}_{\mathbf{e f f}} / \mathbf{E I}_{\mathbf{c}}$ & $\mathbf{M}_{\mathbf{r}} / \mathbf{M}_{\mathbf{y}}$ & $\begin{array}{c}\boldsymbol{\theta}_{\mathbf{y}} \\
(\mathbf{r a d})\end{array}$ & $\begin{array}{c}\boldsymbol{\theta}_{\mathbf{p}} \\
(\mathbf{r a d})\end{array}$ & $\begin{array}{c}\boldsymbol{\theta}_{\mathbf{p c}} \\
(\mathbf{r a d})\end{array}$ & $\begin{array}{c}\boldsymbol{\theta}_{\mathbf{u}} \\
(\mathbf{r a d})\end{array}$ & $\boldsymbol{\lambda}$ \\
\hline $\begin{array}{c}\text { Theory } \\
\text { (AASHTO 2009) }\end{array}$ & 544 & 1.07 & 0.29 & 0.8 & 0.006 & 0.043 & - & 0.049 & - \\
\hline $\begin{array}{c}\text { Haselton } \\
\text { (Haselton 2008) }\end{array}$ & 544 & 1.13 & 0.20 & - & 0.009 & 0.019 & 0.033 & 0.062 & 42 \\
\hline $\begin{array}{c}\text { Biskinis } \\
\text { (Biskinis 2009) }\end{array}$ & 542 & - & 0.19 & - & 0.010 & 0.022 & - & 0.032 & - \\
\hline $\begin{array}{c}\text { PEER/ATC 72-1 } \\
\text { (PEER/ATC 72-1 } \\
\text { 2010) }\end{array}$ & 544 & 1.13 & 0.20 & 0.0 & 0.009 & 0.019 & 0.033 & 0.062 & 24 \\
\hline \begin{tabular}{c} 
This study \\
\hline
\end{tabular} & 544 & 1.13 & 0.20 & 0.2 & 0.009 & 0.019 & 0.033 & 0.062 & $\begin{array}{c}42 \\
2\end{array}$ \\
\hline
\end{tabular}

Some of the shortcomings of the equations proposed by Haselton (Haselton 2008) and Biskinis and Fardis (Biskinis and Fardis 2009) is that they do not include the effect of number of cycles on the column rotation capacity. Moreover, Haselton's equations do not account for the effect of lap-spliced rebars in expected plastic hinge locations. Despite this fact, Haselton's and Biskinis's equation lead to similar plastic rotation capacity $\left(\theta_{\mathrm{p}}\right)$.

Figure 4.5 shows the results using the model parameters summarized in Table 4.2. These plots show the effect of the conventional protocol and the subduction protocol for structures of ductility 8. Protocols with that target ductility were used because the ductility obtained from moment-curvature analysis was equal to 7. Comparing the results from the two protocols it can 
be observed that for structures with high values of $\lambda$, i.e. low rate of strength and stiffness deterioration, the behavior of the column under both protocols is quite similar in terms of rotation capacity, which is considered as the rotation when a reduction in moment capacity of $20 \%$ occurs.

On the other hand, if a high rate of deterioration (low $\lambda$ ) is considered the column under the subduction protocol shows less rotation capacity as compared to the column under the standard protocol. This implies that the faster the rate of deterioration, the more significant the expected effect of number of inelastic cycles on column behavior.
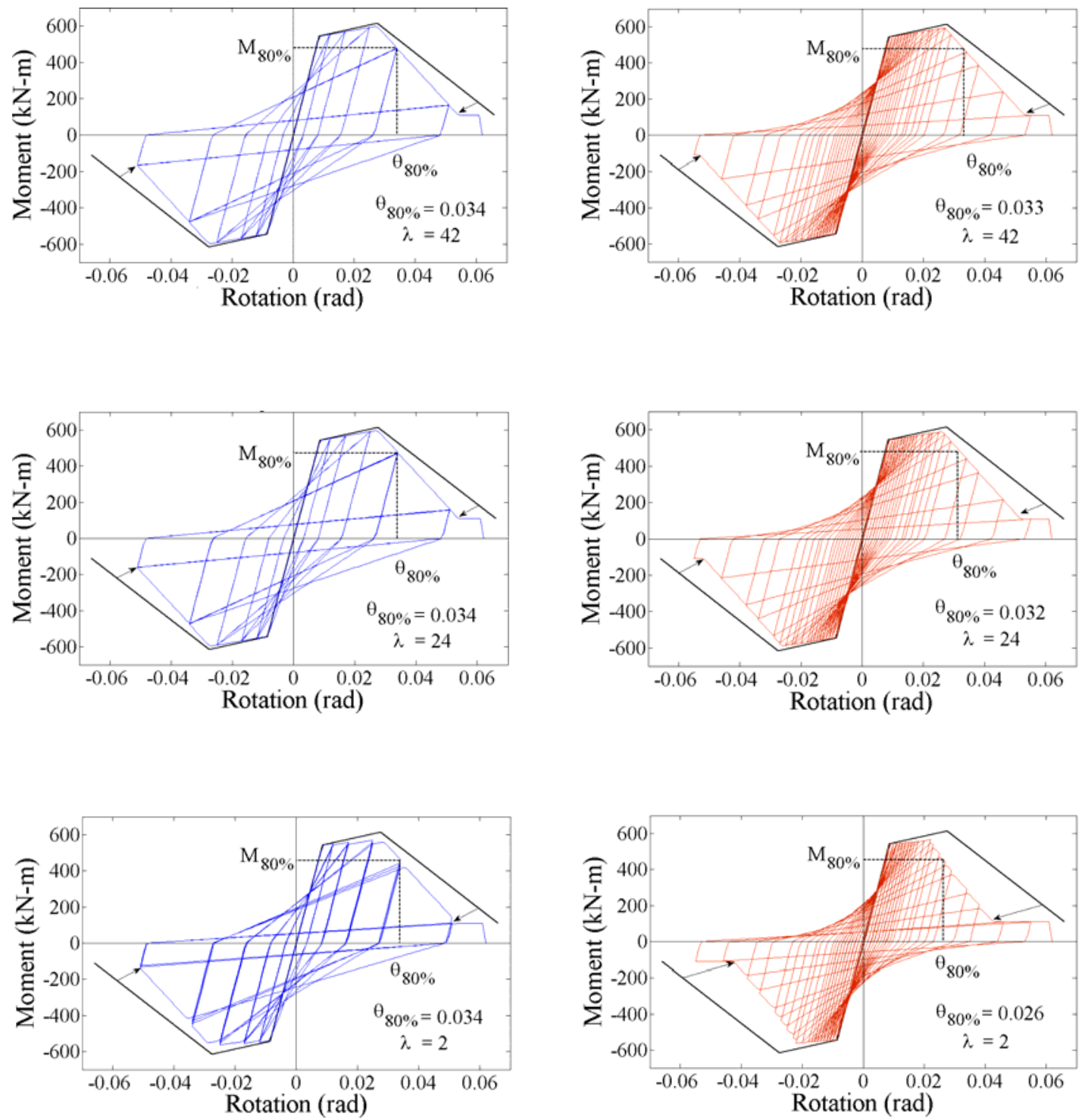

(a)

(b)

Figure 4.5: Effect of loading protocol and model parameters on column response. (a) Standard Protocol. (b) Subduction Protocol 
A high rate of deterioration is expected on pre-1970 columns due to the fact that they were built with lap splices in plastic hinge regions and insufficient transverse reinforcement. Therefore, the behavior of these columns would be highly influenced by subduction mega earthquakes. This result is consistent with experimental and numerical studies, e.g. Ibarra and Krawinkler (Ibarra and Krawinkler 2005b), Borg et al. (Borg et al. 2012), Ou et al. (Ou et al. 2013), Chandramohan, et al. (Chandramohan et al. 2013). In those studies were concluded that structural components' capacity and collapse are influenced by the duration of ground motion and the number of inelastic cycles.

\subsection{EXPERIMENTAL CASE STUDY}

In order to assess the response of RC bridge column that are seismically deficient, cyclic loading protocols can be utilized. This section shows the experimental results of full-scale, square cross section, reinforced concrete bridge columns. The specimens and the laboratory arrangement were intended to model typical Oregon Bridge columns constructed in the 1950s to mid-1970s as illustrated in Figure 4.3. Two different quasi-static cyclic loading protocols were employed to evaluate the effect of the proposed loading protocols shown in Chapter 3.

\subsubsection{Test Program}

The experimental program consisted of two test specimens intended to represent full-scale models of typical RC bridge columns. The specimens were constructed using the same material properties, cross-sectional dimensions and reinforcement ratios. The variable in the testing program was the cyclic loading protocol applied at the top of the columns. The performance of these specimens was intended to reveal vulnerabilities in existing deficient columns subjected to different loading conditions.

\subsubsection{Test Specimens}

In order to simplify the analysis and fabrication of the test specimens, the reinforced concrete column design was idealized as a cantilever column fixed at one end and free on the opposite end. The test setup is representative of the internal loads of a full height column due to the assumed location of the inflection point. A sketch of the prototype column reinforcement detail and cross section is shown in Figure 4.6.

The longitudinal reinforcement in each specimen consisted of four \#10 bars on four corners with \#3 stirrups spaced at 12 inches center to center. Lap splices were located at the base of the test specimens through the incorporation of 4 dowels. The lap splice length was 36 inches, which corresponds to 28 times the diameter of the longitudinal steel reinforcement $\left(28 \mathrm{~d}_{\mathrm{b}}\right)$. All reinforcing steel used to construct the test specimens consisted of Grade 60 deformed bar conforming to the American Society of Testing and Materials (ASTM) designation A615.

Normal weight concrete was used to construct the test specimens with a target 28-day strength of 3500 psi. The concrete cover was 2 inches. Standard compression testing of 6inch by 12-inch concrete cylinders was performed at approximately 7-day intervals up to 
28 days and at the day of test completion. The average of the concrete cylinder tests are shown in Table 4.3.

The columns in the experimental program were given an identification that denoted the column condition ( $\mathrm{A}=\mathrm{As}-\mathrm{built})$ and loading protocol $(\mathrm{SU}=$ Subduction, $\mathrm{CO}=$ Conventional). The identifications for the two columns in the experimental program were A-SU, A-CO.

Table 4.3: Compressive strength of concrete cylinders for deficient square columns

\begin{tabular}{c|c}
\hline Concrete Pour & $\begin{array}{c}\text { Compressive Strength }\left(\mathbf{f}_{\mathbf{c}} \mathbf{c}^{\mathbf{c}} \mathbf{\text { psi }}\right. \\
\mathbf{7 ~ d a y}\end{array}$ \\
\hline 14 day & 2470 \\
\hline 21 day & 2993 \\
\hline 28 day & 3175 \\
\hline $\begin{array}{c}\text { Specimen A-SU } \\
\text { (test day) }\end{array}$ & 3507 \\
\hline $\begin{array}{c}\text { Specimen A-CO } \\
\text { (test day) }\end{array}$ & 4171 \\
\hline
\end{tabular}

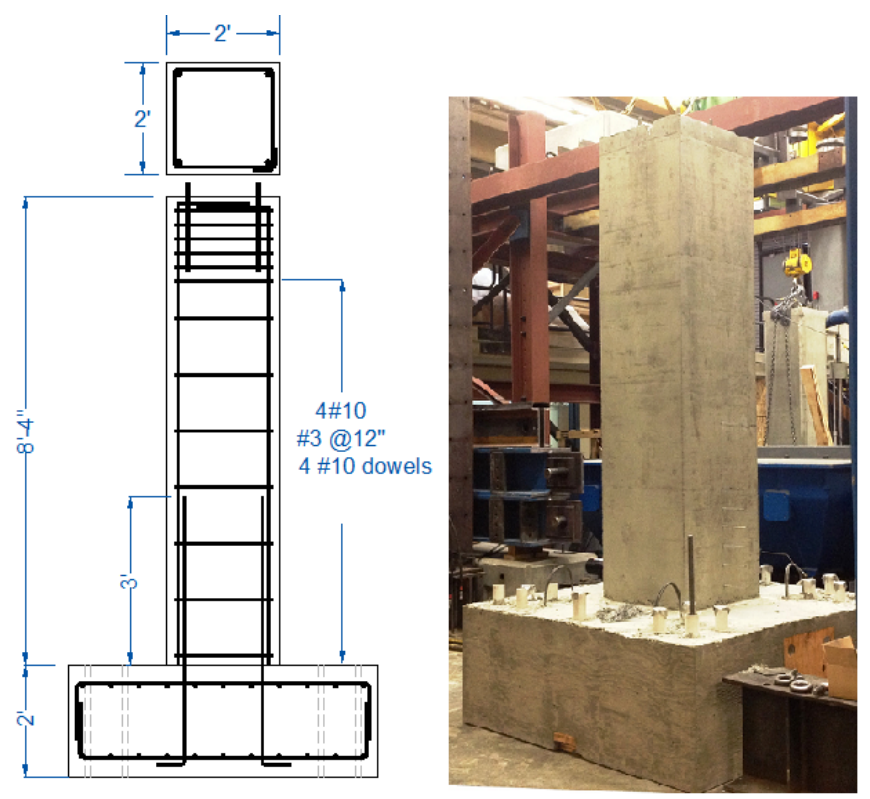

Figure 4.6: Geometry and reinforcement of RC Bridge column specimens

\subsubsection{Test Setup and Instrumentation}

The columns were tested in the upright position. The top of the column was free to translate and rotate. The cyclic lateral loading was applied through a horizontal hydraulic actuator connected to steel beam on top of the column. The lateral force was applied under displacement control. The applied lateral load was reacted against a steel reaction 
frame connected to the laboratory strong floor. Load cells were used to monitor the applied load during testing.

Axial load on the column was applied in an effort to simulate the dead loads from the superstructure. The applied axial load was approximately 7 percent of the axial strength (Agf'c). This load was applied through four high-strength rods and four hydraulic rams, which were attached to the horizontal steel beam on top of the columns. Load cells were used to monitor the applied axial load during testing. The footing was secured to the laboratory floor with post-tensioning rods. The rods were placed on four corners of the column. A schematic representation of the experimental test set up is shown in .

Failure of the specimen was defined as a $20 \%$ drop in peak lateral load. The yield displacement of each specimen was predicted from moment curvature analysis and was corrected during the test. The experimental yield displacement $\left(\delta_{\mathrm{y}}\right)$ was established by using the ratio of the theoretical force at which the concrete reaches a strain of 0.004 to the experimental elastic stiffness $\left(\mathrm{K}_{\mathrm{e}}\right)$, which is calculated as the ratio of the theoretical first yield force $\left(\mathrm{V}_{\mathrm{i}}\right)$ to the displacement measured experimentally $\left(\delta_{\mathrm{y}}{ }^{\prime}\right)$ as illustrated in Figure 3.8

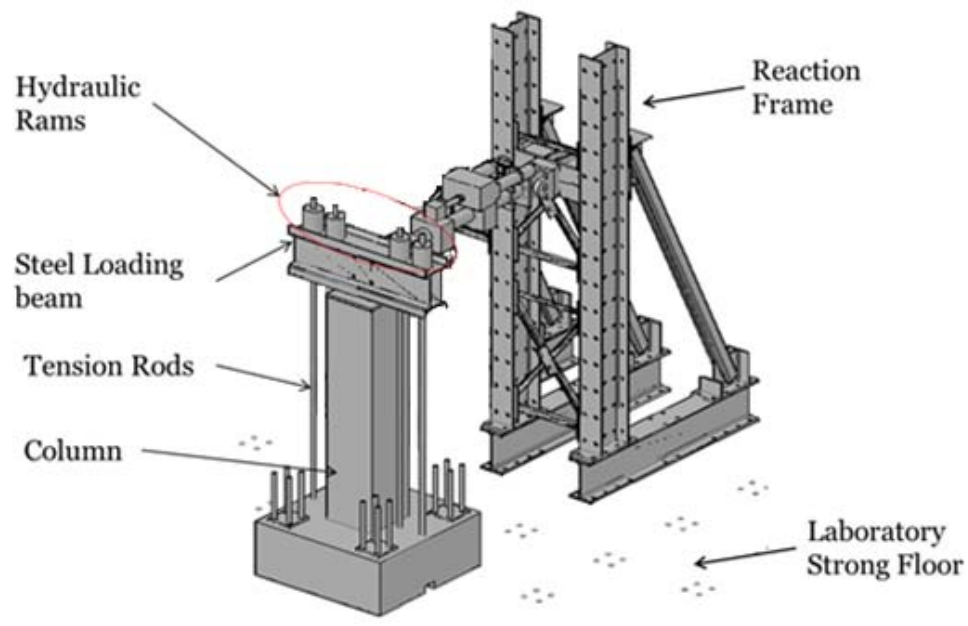

Figure 4.7: Test setup configuration

In order to analyze the response of the specimens subjected to cyclic lateral loading, the columns were fully instrumented via strain gages and linear variable displacement transducers (LVDTs) as shown in Figure 4.8 and Figure 4.9, respectively. Strain gages were used to measure the strain at specific points in the specimens. A total of 12 strain gages were mounted on the longitudinal reinforcing bars, ( 2 on the continuous longitudinal bars and 10 on the dowels). All strain gages were placed at and below the plastic hinge which was theoretically calculated as 26 inches. Installation of all strain gages was performed prior to the placement of the concrete. LVDTs were used to measure displacements of specified points on the specimen. 

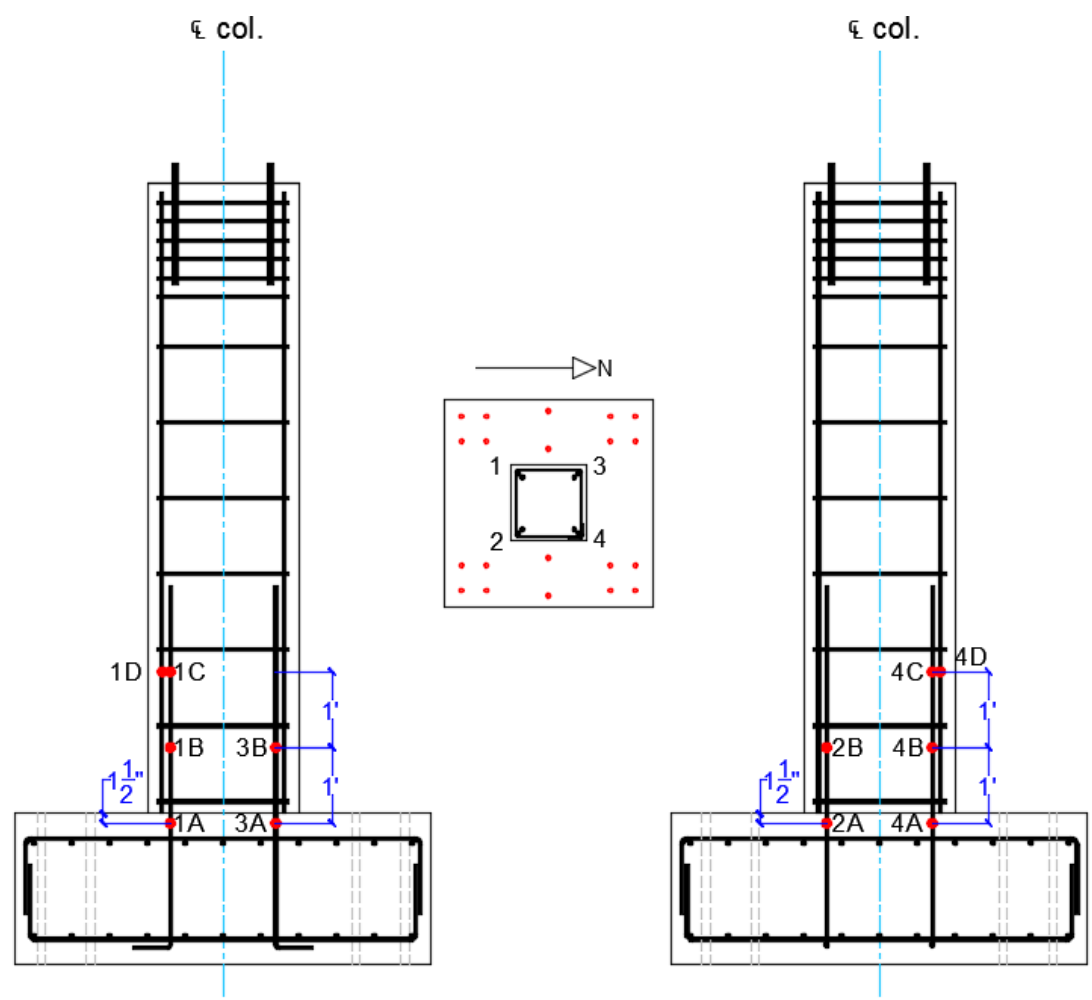

Figure 4.8: Location of strain gages acol.

¿col.

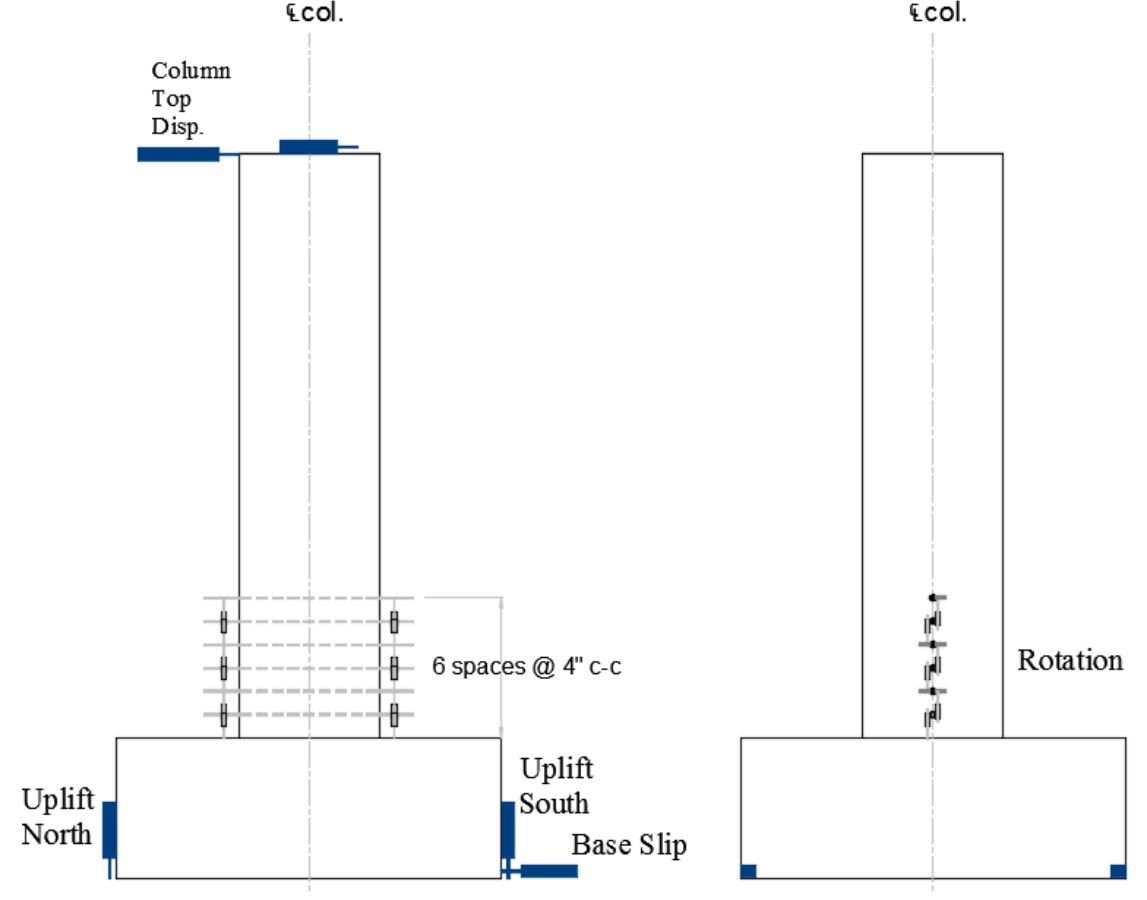

Figure 4.9: Location of LVDTs 


\subsubsection{Cyclic Loading Protocol}

As in the numerical case study, for this experimental program two types of loading protocols were used, namely, conventional cyclic loading protocol and subduction zone loading protocol. The modified New Zealand protocol was used as the conventional cyclic loading protocol and the proposed protocol for structures of ductility 8 and fundamental period of $0.5 \mathrm{sec}$ was used as the subduction zone loading protocol. Protocols with that target ductility were used because the ductility obtained from moment-curvature analysis of the column in study was close to 8 . Both protocols used in the experimental study are shown in Chapter 3.

\subsubsection{Test Results}

\subsubsection{Column A-CO}

In this column the modified New Zealand conventional protocol was utilized as shown in Figure 3.1(b). The column was checked for cracks before the beginning of the experimental test in an effort to observe any initial retraction cracking. During the test the first crack was initiated as a horizontal crack near the base of the column at small lateral displacements $(<0.3$ inches). New horizontal cracks were observed on the column faces at the base of the column after the first peak displacements. As the test progressed numerous horizontal cracks were visible up to the mid-height of the column as shown in Figure 4.10(a).

For column A-CO the primary mode of failure was crushing of concrete at the base of the column in conjunction with buckling of dowels as illustrated in Figure 4.10(b) and (c). The buckling of the dowels was observed once the cover of the concrete at the base of the column was completely crushed.

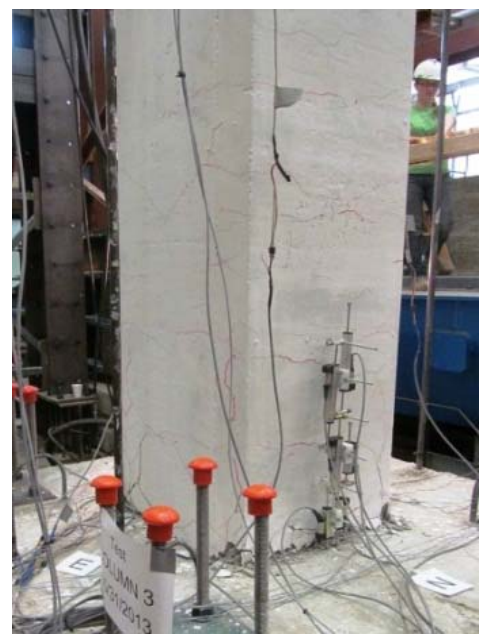

(a)

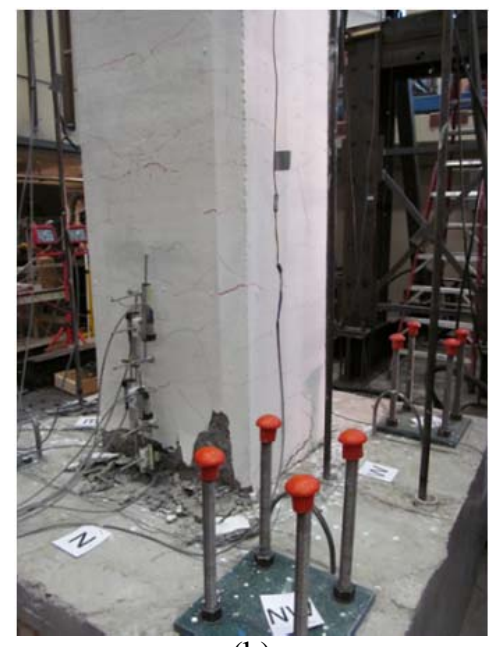

(b)

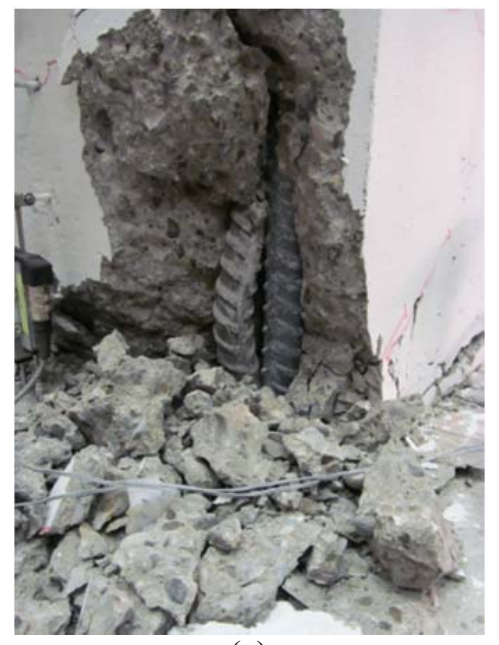

(c)

Figure 4.10: Damage progression in Column A-CO. (a) Horizontal cracks, (b) Crushing of concrete, (c) Bar buckling 
The lateral load vs. displacement hysteresis curve for the column A-CO is illustrated in Figure 4.11. Yield displacement of the specimen was initially calculated as 0.73 inches. However, this yield displacement was corrected during the test in an effort to accommodate the actual response of the test specimen. In order to make this correction the procedure described in Chapter 3 and depicted in Figure 3.8 was utilized. The experimental yield displacement was 1.06 inches, which corresponds to a $45 \%$ increase with respect to the theoretical value.

The hysteretic behavior of this specimen showed a moderate to high ductile behavior by reaching a displacement ductility above 4.5 and 6 in the positive and negative direction, respectively. Column A-CO showed a hysteretic response typical of flexural behavior with slightly pinching behavior. As expected in the elastic range, slight strength degradation between cycles at the same amplitude can be observed before the occurrence of the yield displacement. Cycles at the same amplitude after yielding showed notorious strength and stiffness degradation.

The peak lateral load was -46 kip and occurred at a lateral displacement of approximately -5.6 inches. The specimen exhibited a significant decrease in lateral strength after the peak lateral load because of a noticeable crushing of concrete at the base of the column. Failure of the specimen was assumed when the applied lateral load dropped below $80 \%$ of the peak load. Displacements of 6 inches (ductility 5.7) and -7.75 in (ductility 7.3) were computed as the failure displacements in the positive and negative direction, respectively.

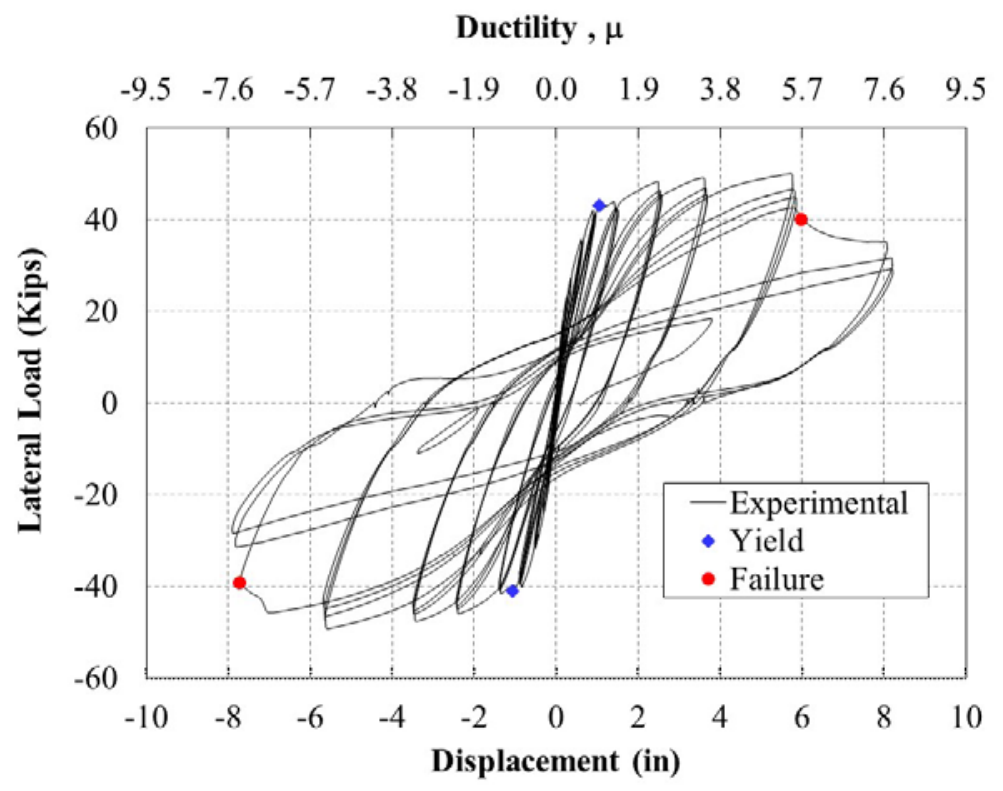

Figure 4.11: Load vs. Displacement curve of column A-CO 


\subsubsection{Column A-SU}

The proposed protocol for structures of ductility 8 and fundamental period of $0.5 \mathrm{sec}$, as shown in Figure 3.7(a), was utilized to test Column A-SU. This column as the previous column was checked for cracks before the beginning of the experimental test in an effort to observe any initial retraction cracking. During the test the first crack was initiated as a horizontal crack near the base of the column at small lateral displacements $(<0.2$ inches $)$. New horizontal cracks were observed on the column faces at the base of the column after the first peak displacements. As the test progressed numerous horizontal cracks were visible up to the mid-height of the column as shown in Figure 4.12 (a). Vertical cracks consistent with lap splice failure started showing at a displacement of 1.8 inches and the cracks width opened up to $>2.0 \mathrm{~mm}$ at a displacement of 4inches as shown in Figure 4.12(b). The concrete above the lap splice length showed only minimal signs of vertical cracking.

For column A-SU the primary mode of failure was lap splice failure followed by crushing of concrete at the base of the column and buckling of dowels as illustrated in Figure 4.12 (b) and (c). The buckling of the dowels was observed once the cover of the concrete at the base of the column was completely crushed.

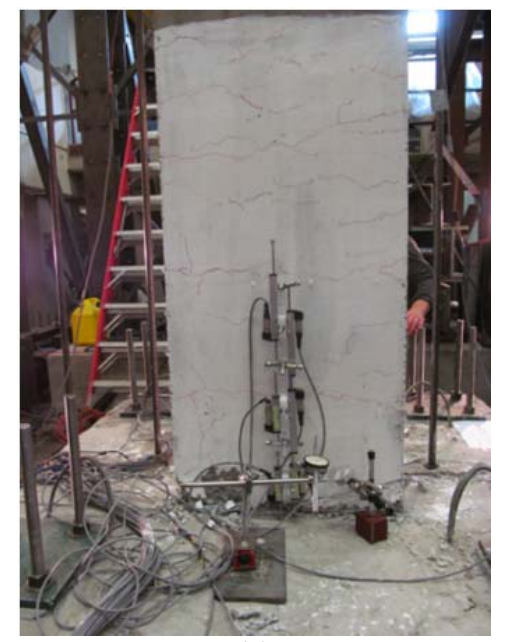

(a)

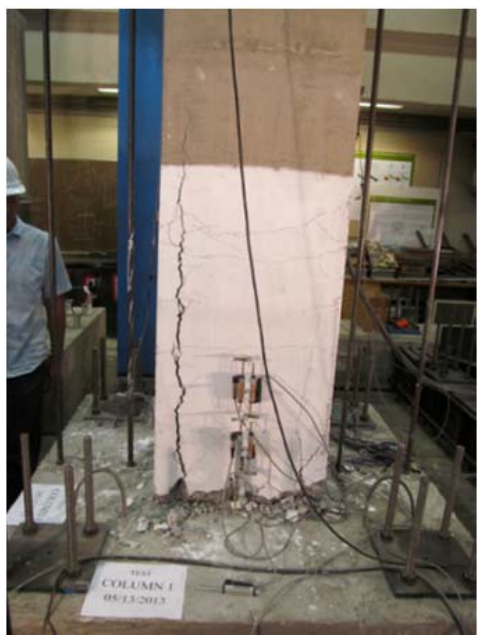

(b)

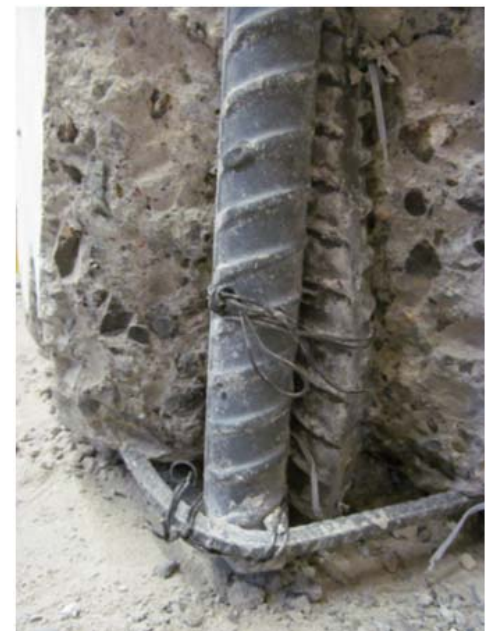

(c)

Figure 4.12: Damage progression in Column A-SU. (a) Horizontal cracks, (b) Crushing of concrete, (c) Bar buckling

The lateral load vs. displacement hysteresis curve for the column A-SU is illustrated in Figure 4.13. Yield displacement of the specimen was initially calculated as 0.73 inches. However, this yield displacement was corrected during the test in an effort to accommodate the actual response of the test specimen. In order to make this correction the procedure described in Chapter 3 and depicted in Figure 3.8 was utilized. The experimental yield displacement was 1 inch, which corresponds to a $37 \%$ increase with respect to the theoretical value. 
The hysteretic behavior of this specimen showed a moderate ductile behavior by reaching a displacement ductility of 4.2 and 5.7 in the positive and negative direction, respectively. Column A-SU showed an initial hysteretic response typical of flexural behavior.

Minimum strength degradation was noticed up to the peak load, which was computed as 44 kip in the positive direction and -46 kip in the negative direction and occurred at a lateral displacement of approximately 3.7 and -4.7 inches, respectively. The specimen exhibited a significant decrease in lateral strength after both peak lateral loads because of a vertical crack that was attributed to lap splice failure. Failure of the specimen was assumed when the applied lateral load dropped below $80 \%$ of the peak load.

Displacements of 4.2 inches (ductility 4.2 ) and -5.7 in (ductility 5.7) were computed as the failure displacements in the positive and negative direction, respectively.

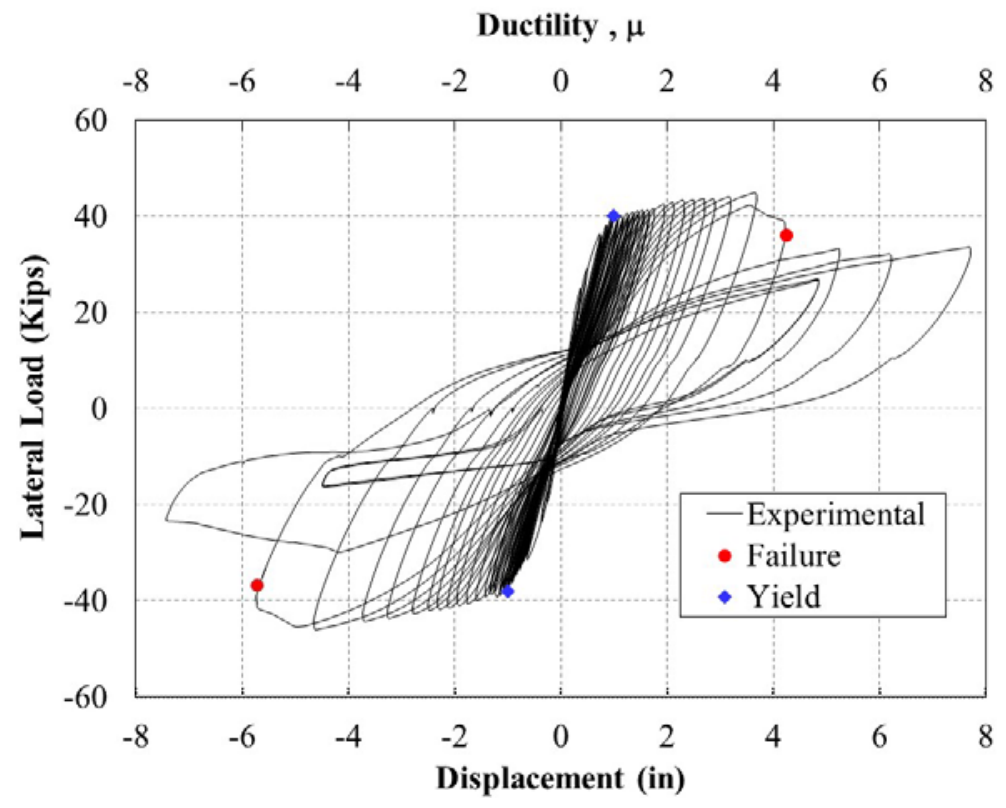

Figure 4.13: Load vs. Displacement curve of column A-SU

\subsubsection{Analysis of Results}

\subsubsection{Comparison to analytical results}

Moment-curvature curves for the columns were obtained by using the Section Designer capability in SAP2000, which uses Caltrans specifications. The moment-curvature analysis was done by assuming the Mander concrete parametric stress-strain curve for the unconfined and confined concrete (Mander 1984) and the Park parametric stress-strain curve for reinforcing steel. The conversion from moment-curvature to load-displacement was done by using a lumped plastic hinge model assuming a curvature and deflection relationship for a reinforced concrete cantilever column developed by Paulay and Priestley (Paulay and Priestley 1992). Details of this analysis are presented in the appendices. 
The comparison between experimental and analytical envelope results is shown in Figure 4.14 and Figure 4.15. The column A-CO, that was tested using a conventional loading protocol, showed an excellent fitting between the experimental and analytical load-displacement envelope curves. On the contrary, column A-SU, which was tested using a protocol that aimed to mimic the demand imposed by subduction zone mega earthquakes, showed less strength and displacement capacity as compared to the analytical results.

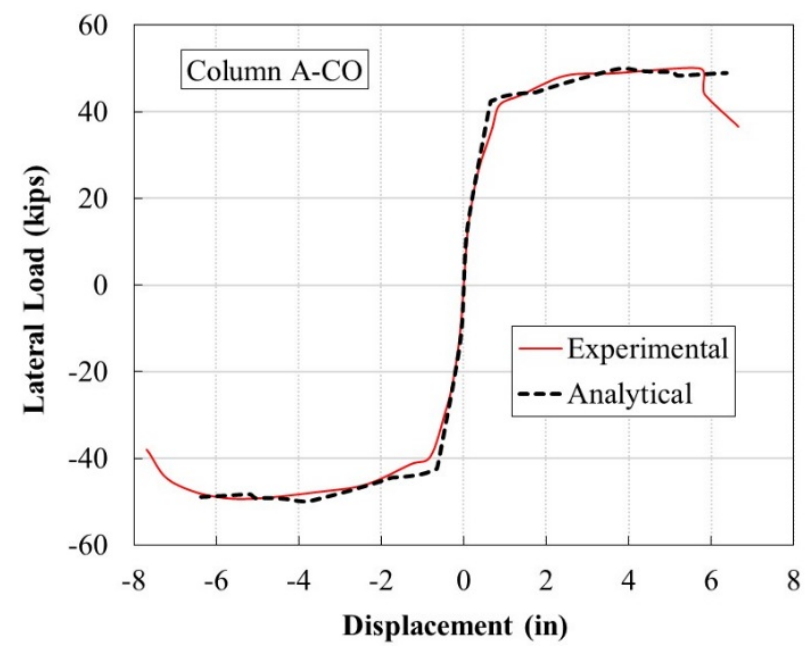

Figure 4.14: Experimental vs. analytical envelope curves for column A-CO

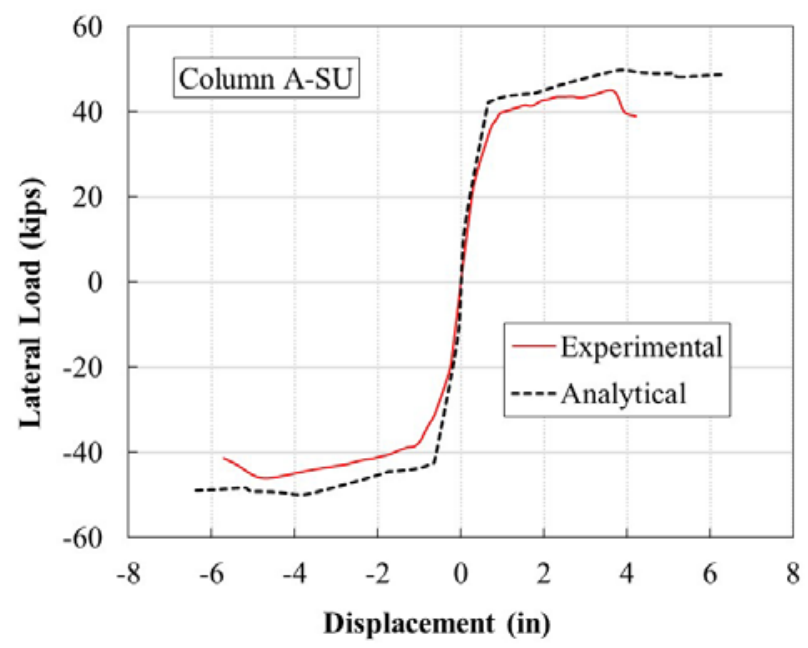

Figure 4.15: Experimental vs. analytical envelope curves for column A-SU

\subsubsection{Comparison between column results}

The column A-SU tested under the subduction loading protocol showed less strength and deformation capacity as compared to the column A-CO tested under the conventional loading protocol. The fact that column A-SU had less strength and deformation capacity 
was attributed to the increasing number of low amplitude inelastic cycles that the column undergoes by using the subduction protocol. This increase in the number of inelastic cycles generated vertical cracks at an early stage of the test that culminated in lap splice failure. This result has direct implications in the design of retrofit measures for existing $\mathrm{RC}$ bridge columns since one would expect less capacity for columns subjected to subduction zone earthquakes and is consistent with the numerical case study and with previous experimental studies (Takemura and Kawashima 1997), (Kunnath et al. 1997), (Ou et al. 2013). Moreover, the result implies that the common assessment of existing bridges by doing pushover analyses might overestimate the actual strength and deformation capacity of RC bridge superstructures.

Despite the seismic detailing deficiencies, the hysteretic response curves showed that both columns had moderate ductility and energy dissipation through nonlinear behavior. The moderately ductile performance is likely a result of a low axial column load $\left(0.07 \mathrm{f}^{\prime} \mathrm{A}_{\mathrm{g}}\right)$ and a relative long lap splice $\left(28 \mathrm{~d}_{\mathrm{b}}\right)$. Even though, this moderate ductility was unexpected, similar results were obtained by El Gawady et al. (El Gawady et al. 2010) for typical deficient columns built prior 1971 in the State of Washington.

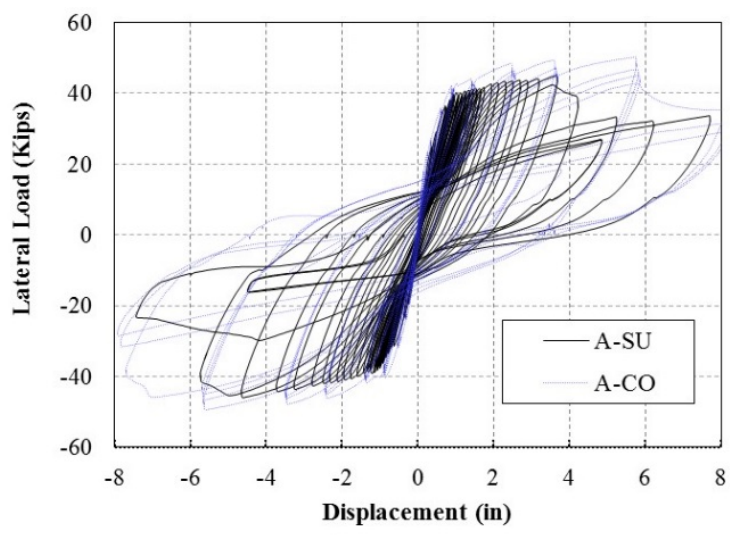

(a)

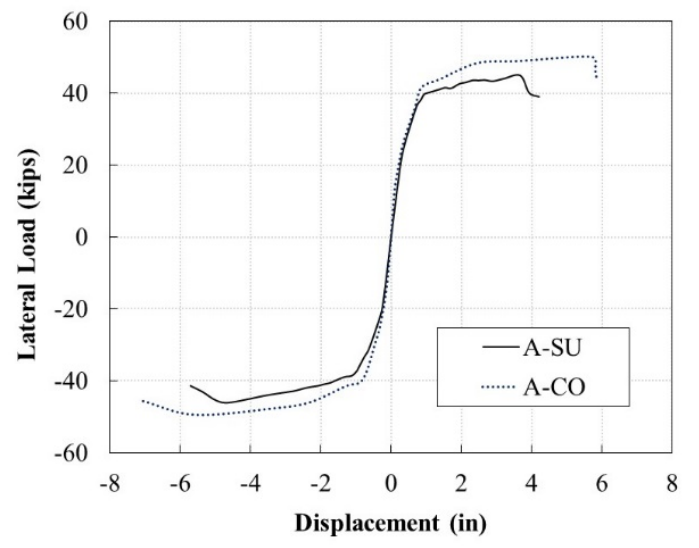

(b)

Figure 4.16: Hysteretic and envelope response comparison

\subsubsection{Stiffness Degradation}

The stiffness degradation is an important property in RC bridges subjected to seismic events since it changes the effective natural period of the structure. Stiffness degradation can be attributed to concrete nonlinear behavior caused by flexural and shear cracking, slippage of steel reinforcement, etc.

In this study, the normalized stiffness, represented as the secant stiffness divided by the yield stiffness, at different displacement ductilities, was utilized as the stiffness degradation parameter. In terms of stiffness degradation both test specimens showed similar stiffness degradation up to a displacement ductility 4 , and there was no significant effect of the loading protocol applied on the stiffness degradation up to that ductility as depicted in Figure 4.17. However, for ductilities above 4, the column A-SU showed a higher stiffness degradation that is concordant with the increasing number of inelastic 
cycles imposed by the subduction zone protocol and the lap splice failure seen during the test.

Gulkan and Sozen (Gulkan and Sozen 1974) proposed a relation between the displacement ductility $(\mu)$ and the secant stiffness $\left(\mathrm{k}_{\mathrm{sec}}\right)$ as follows:

$$
\frac{k_{\mathrm{sec}}}{k_{y}}=\frac{1}{\mu} \leq 1
$$

Where, $\mathrm{k}_{\mathrm{y}}$ is the secant stiffness at displacement ductility 1 . The test results closely follow the predictions obtained using Eq. (4.1).

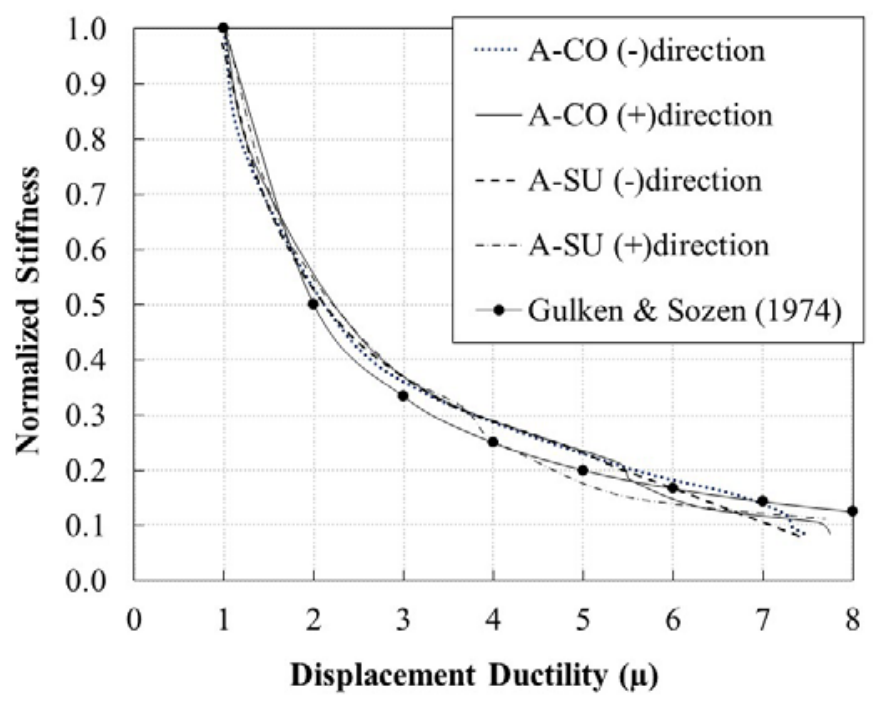

Figure 4.17: Stiffness degradation

\subsubsection{Rotation Capacity}

12 LVDTs located at the base of the columns at heights 4", 8", 12", 16", 20", and 24" respectively from top of footing, as depicted in Figure 4.9, were used to measure the rotation of the specimen. As can be seen from Figure 4.18, the rotation for both columns was concentrated at the base, which was a result of a rocking behavior observed during the tests. Furthermore, the rotation capacity of 0.05 to $0.06 \mathrm{rad}$ is consistent with the rotation capacity calculated in the numerical case study shown in Table 4.2. 

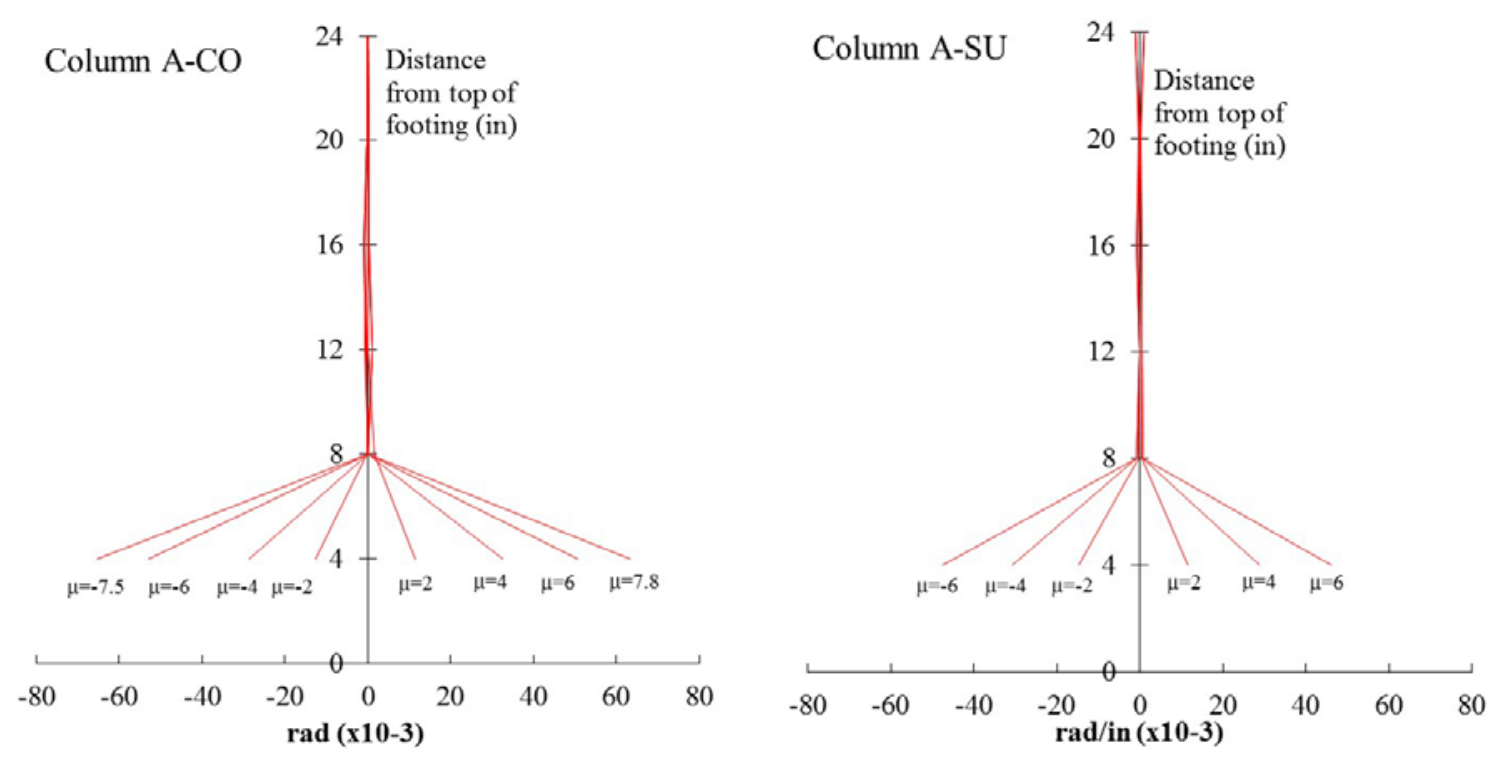

Figure 4.18: Rotation capacity

\subsection{SUMMARY}

A representative pre-1970 lightly reinforced and lap-spliced bridge column was studied to observe the effect of the application of two different loading protocols on the behavior of seismically deficient reinforced concrete bridge columns. Observations based on numerical and experimental results of applying cyclic loading protocols on seismically deficient RC columns can be summarized as follows:

- Numerical results showed the importance of modeling the rate of strength and stiffness deterioration in RC bridges. This is of paramount importance in regions susceptible to be struck by subduction zone mega earthquakes since the faster the rate of deterioration the more significant the expected effect of number of inelastic cycles on column behavior.

- The experimental results of this study indicate that square columns present in bridges built before 1971 in the Pacific Northwest have unexpected deformation capacity. This moderately ductile performance of the as-built columns was likely a result of a low axial load level (0.07 f'c Ag) and low longitudinal steel reinforcement ratio $(\rho=$ $1.2 \%)$.

- The experimental study also demonstrated that subduction loading protocols reduce the displacement ductility capacity of reinforced concrete columns and change the failure mode of columns. The primary mode of failure for the column tested under subduction loading protocol was by lap splice failure. On the contrary, Column A$\mathrm{CO}$, which was tested using a conventional cyclic loading protocol, failed showing crushing of concrete as a primary mode of failure. 
- Despite the fact that the conventional protocol contains a higher number of large inelastic excursion, results showed that the use of the subduction protocol can highly influence the response of deteriorating components. Even though, more extensive analytical and experimental studies are needed to reach broader conclusions, the assessment of bridge columns through representative testing load protocols would play a key role in the future establishment of limit states and acceptance criteria to be applied in performance-based seismic design of bridge columns. 


\subsection{STATE OF THE ART ON RETROFIT MEASURES}

\subsection{GENERAL}

Reinforced concrete bridge substructures constructed before 1970 are commonly deficient in flexural ductility and shear strength as those bridges were designed primarily for gravity loads with little or no design consideration to seismic resistance. RC bridge bents lack sufficient steel reinforcement to provide satisfactory performance in a major seismic event. Typically, No. 3 or No. 4 hoops at 12 inches on center were provided in columns regardless of the column crosssectional dimensions. The stirrups were anchored by $90^{\circ}$ hooks with short extensions and intermediate ties were seldom used. RC bridges built prior to 1970 also have inadequate lap splices in potential plastic hinge zones since dowels were usually extended between 20 to 40 longitudinal bar diameters $\left(\mathrm{d}_{\mathrm{b}}\right)$ from the foundations. This detail may cause a considerable reduction on column ductility and a rapid loss of flexural and axial strength. Furthermore, the foundations constructed at that time were not design with a capacity design in mind. As a result, foundations are highly susceptible to brittle flexural and shear failures since they do not contain neither top longitudinal reinforcement nor transverse reinforcement.

All these details contribute to bridge' deficiency and make these structures highly vulnerable to a major seismic event. This vulnerability of pre-1970 bridges was especially evident in the 1971 San Fernando, 1989 Loma Prieta earthquake, and the 1994 Northridge earthquake as shown in Figure 5.1, Figure 5.2 and Figure 5.3, respectively. Further, the recent occurrence of subduction zone mega earthquakes in Chile and Japan has demonstrated how vulnerable RC bridges are when subjected to major seismic events as shown in Figure 5.4.

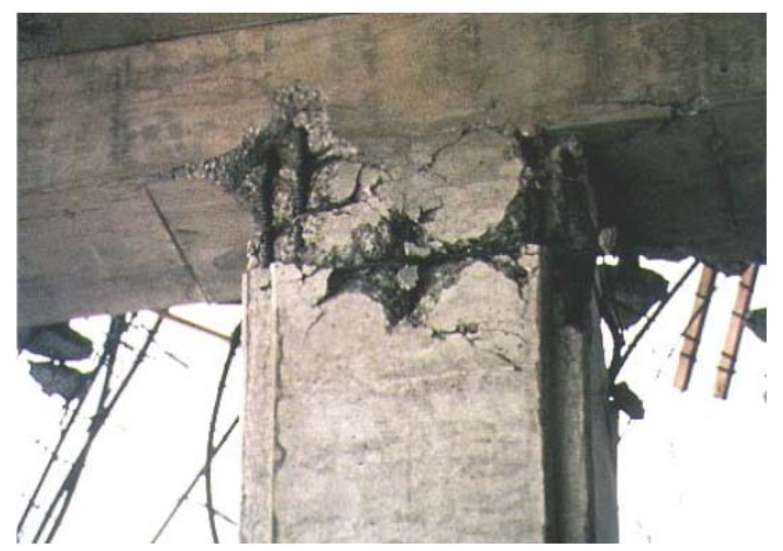

Figure 5.1: Poorly Confined Column in 1971 San Fernando Earthquake 


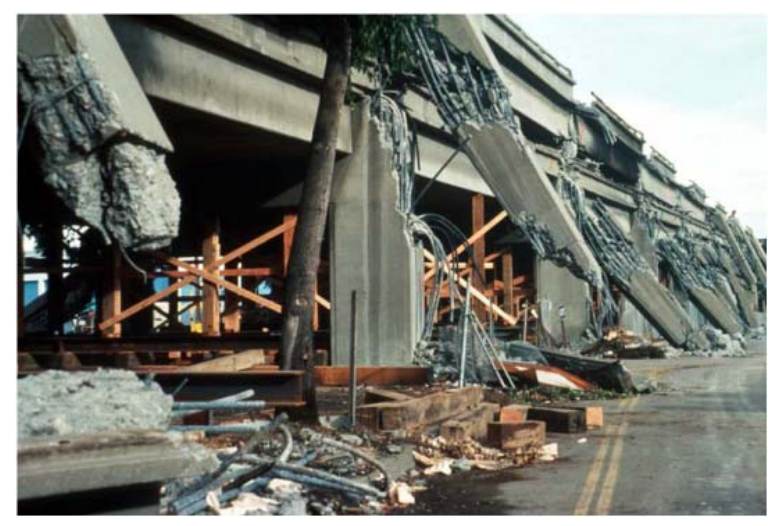

Figure 5.2: Collapsed Cypress street viaduct in Oakland during the 1989 Loma Prieta Earthquake (USGS and Wilshire 1989)

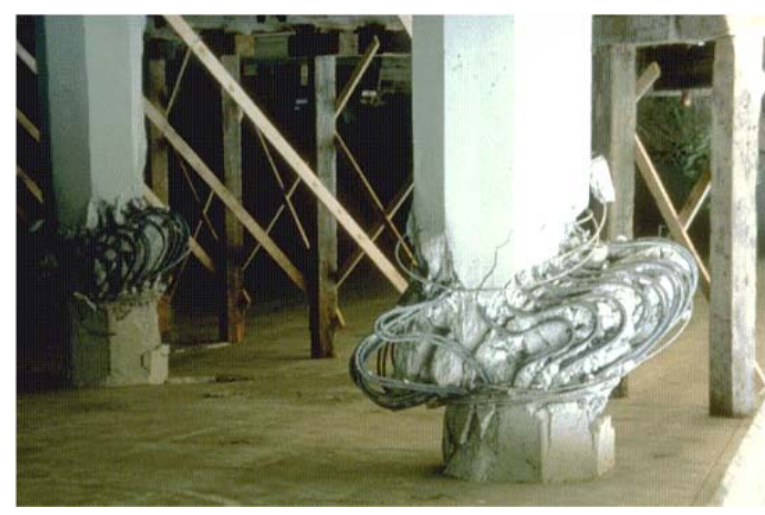

Figure 5.3: Damage in RC columns on the Simi Valley Freeway during the 1994 Northridge Earthquake (Teng 1994)

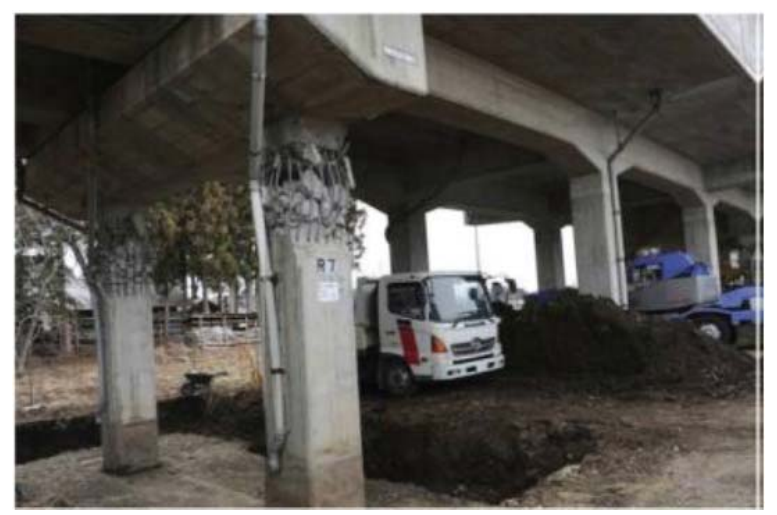

Figure 5.4: Damage in Nakasone viaduct during 2011 Japan earthquake (Kawashima et al. 2011)

The ability of structures to achieve adequate deformation capacity plays a significant role in the prevention of structural failures in seismic events. The deformation capacity of existing bridges can be enhanced by modifying certain substructure elements and connections. Various retrofit and strengthening techniques have been developed and implemented. For example, bridge columns can be retrofitted using various techniques including reinforced concrete jacketing, steel 
jacketing, active confinement by prestressing wire, and composite fiber/epoxy jacketing, etc. Techniques to retrofit other bridge members have also been developed (FHWA 2006), (Wright et al. 2011) as shown in Figure 5.5 and Figure 5.6 for bent caps and footings, respectively.

The main goal of any seismic retrofit measure is to minimize structural collapse, while meeting certain performance requirements. Nowadays, the number of available retrofit measures has increased markedly as a result of extensive analytical and experimental studies. In this chapter conventional and emerging retrofit measures capable of improving the seismic resistance of deficient RC bridge substructures built before 1970 in the State of Oregon are presented and discussed. It is worth noting that retrofit measures that aim to decrease the force demand on the bridge through the implementation of isolation devices is not discussed in this study since the objective of the retrofit proposed in this study was to decrease displacement demands.

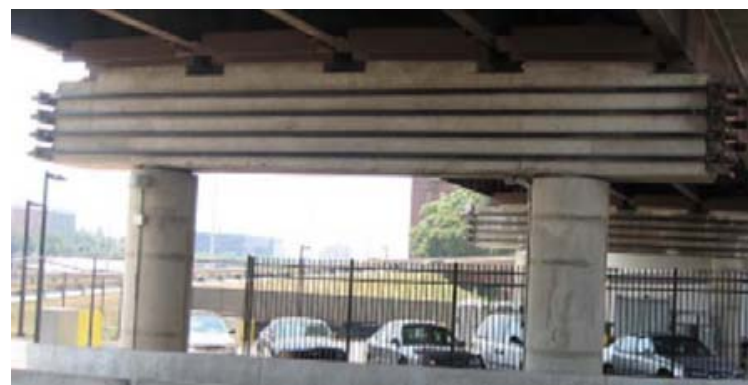

(a)

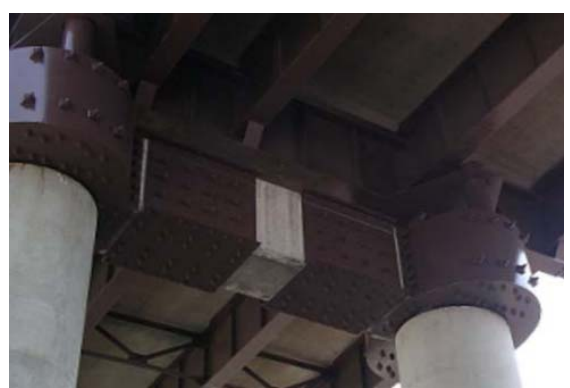

(b)

Figure 5.5: Retrofit measure for bent caps. (a) Posttensioned rods. (b) Steel Jackets. (Wright et al. 2011)
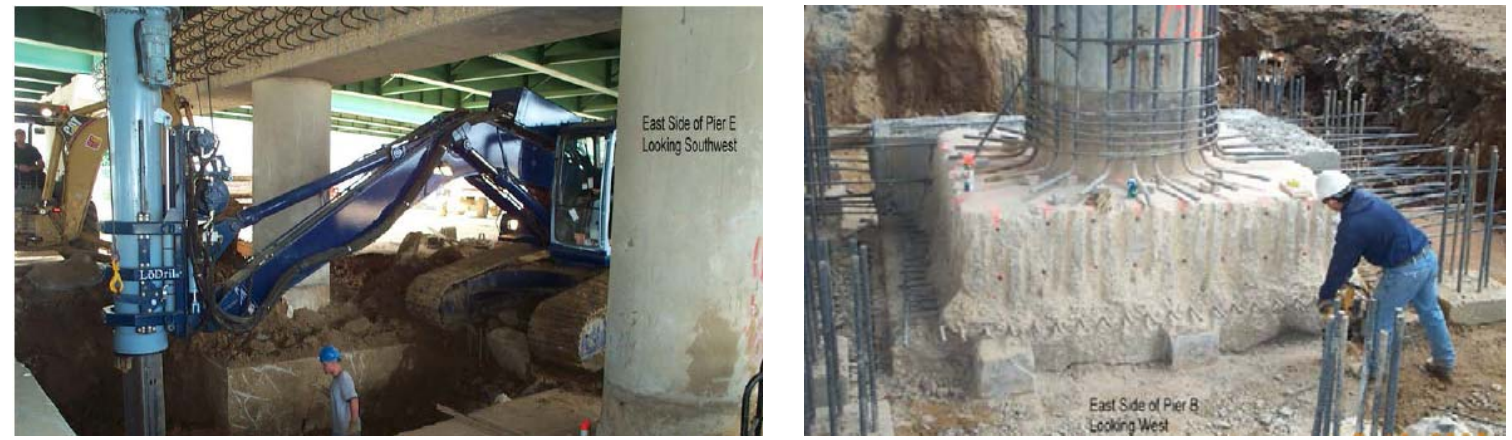

Figure 5.6: Footing overlay on Hernando-Desoto Bridge (photo: R.A. Imbsen)

\subsection{RETROFIT MEASURES FOR RC COLUMNS}

Previous research studies have shown that the main factors causing RC bridge column failures are their insufficient flexural and shear capacity added to a lack of concrete confinement and lap splice at expected plastic hinge regions at the base of the columns. (Chai et al. 1991),

(Dyngeland 1998). In order to overcome these deficiencies many retrofitting systems have been developed and experimentally validated. Most of the retrofit measures for RC columns aim to improve the column ductility, shear strength and provide confinement by "Jacketing" the column through the use of various materials. These methods have now been implemented on a large 
number of deficient bridges throughout seismic regions and have helped preventing bridge failures in major seismic events. (Kawashima et al. 2011), (Hoshikuma and Guangfeng 2013).

\subsubsection{Concrete Jacketing (Concrete Overlays)}

Concrete jacketing was the first method to be employed as a jacketing retrofit measure in practice since 1980. Its application was mainly for RC buildings in Japan as reported by Hayashi et al. (Hayashi 1980) and Sugano (Sugano 1981). The concrete jacketing retrofit measure consists of encasing the existing reinforced concrete column with a jacket of concrete reinforced with longitudinal steel and drilled and grouted dowels or welded wire fabric. Its application follows the same principles as any concrete design. Concrete jackets have been primarily used for retrofitting existing RC columns in buildings. However, its use in bridges has been limited mainly because its labor intensive and more efficient retrofit measures has been implemented for those structures. An example of concrete jacketing for bridge columns is shown in Figure 5.7.

Multiple experimental investigations and field applications have shown that concrete jackets significantly improve the strength and stiffness of as-built columns (Hayashi and Fukuhara 1980), (Teran and Ruiz 1992), (Rodriguez and Park 1994), (Bracci et al. 1995). However, an increase in column flexural strength results in increased shear capacity, and consequently in an increased force transfer to cap beams and footings (FHWA 2006). Therefore, special attention needs to be placed in those components since undesirable effects can be generated on bridge performance.

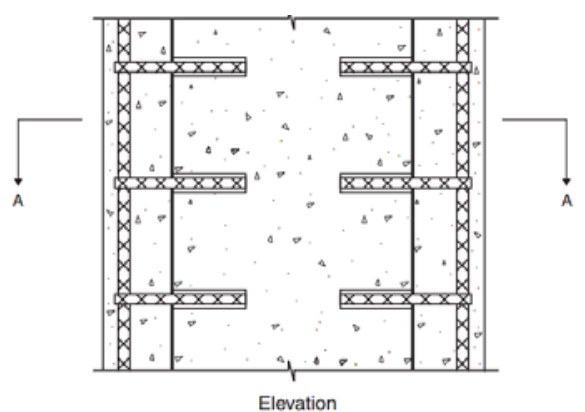

(a)

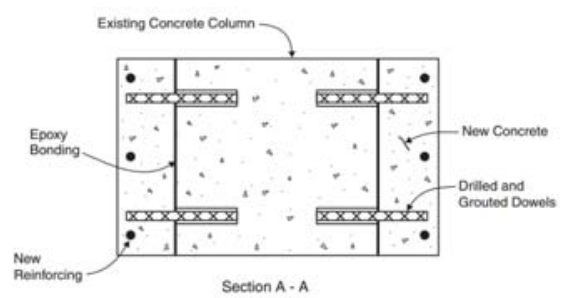

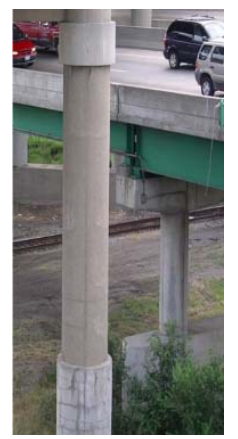

(b)

Figure 5.7: Concrete Jacketing. (a) Concrete overlay squematic (FHWA 2006), (b) Concrete jacketing of a column in Illinois (Poplar Street Complex)

\subsubsection{Steel Jacketing}

Steel jacketing was originally developed as a retrofit measure for circular columns in the early 90s and since then it has been largely utilized for the retrofitting of deficient columns in California (Caltrans 1996) and lately throughout the United States.

Previous research studies (Chai et al. 1991) have shown that steel jacketing is an effective retrofit technique for seismically-deficient concrete columns. Based on satisfactory laboratory 
results, steel jackets have been employed to retrofit both circular and rectangular columns as shown in Figure 5.8. For circular columns, the recommended procedure is to cover the entire column or portion of it with two steel plate half-shells slightly oversized for easy installation leaving two vertical seams that are later welded. The gap between the column and the jacket is filled with grout to provide the required confinement action. An additional gap is required when the full height of the column is steel jacketed in order to minimize flexural strength enhancement, which may cause an undesirable increase in the forces developed in adjacent members. With rectangular columns, the recommended procedure is to use an elliptical shape jacket, which provides continuous confining action similar to that for a circular column. For both cases, the steel jacket acts as a passive confinement, i.e. the steel jacket only provides confinement once the concrete column starts expanding under compressive stresses that induces circumferential stresses in the steel shell. Detailed design guidelines for steel jacketing are found in the Seismic Retrofitting Manual for Highway Structures (FHWA 2006) and in Chai et al. (Chai et al. 1992). Steel jackets are effective in enhancing the confinement of the column, thus increasing the compression strength and strain. Additionally, these jackets can improve column ductility by eliminating the brittle shear and bond mode of failure attributed to a lack of shear reinforcement and lap splices at the base of columns, respectively. The failure mode is consequently shifted to a flexural mode. An undesirable effect of column jacketing is that its application may increase column stiffness. Studies have suggested that this increase could be approximately $10-15 \%$ for the case of partial height jackets (Chai et al. 1991) and $20-40 \%$ for the case of full height jackets (Priestley et al. 1996). This increase in column stiffness may attract higher forces in the bridge and result in increased forces into bent caps and footings.

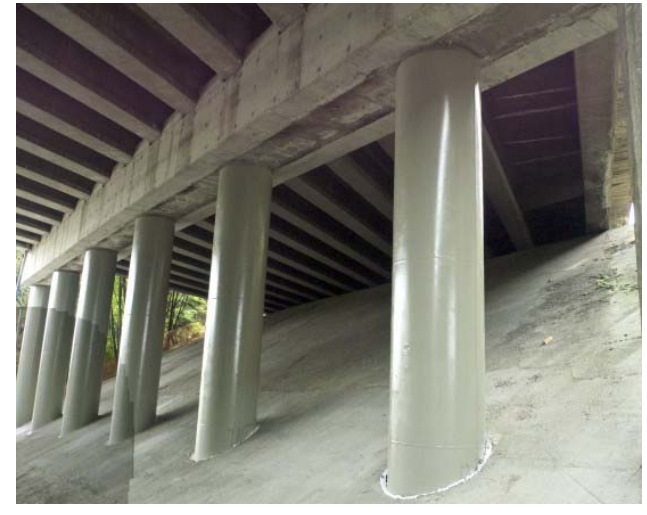

(a)

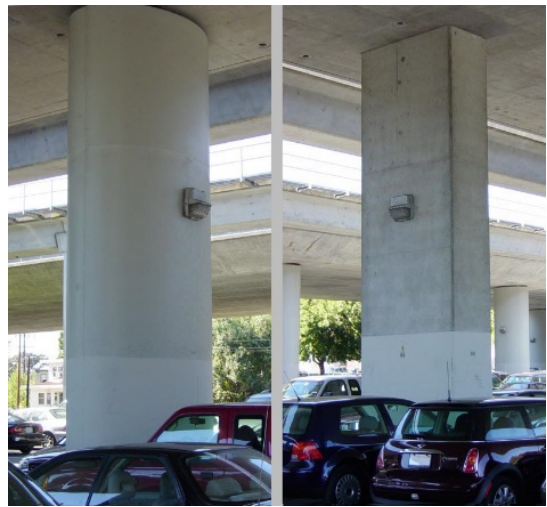

(b)

Figure 5.8: Steel Jacketing for; (a) circular columns in bridge along Interstate 5, Seattle (photo: Lucia Bill), (b) rectangular column in bridge State Route 24 eastbound, San Francisco. Left: retrofitted column. Right: As-built column (photo, Leonard G.)

\subsubsection{Composite Jacketing}

Retrofit measures using composite materials has considerable grown since the past decade. The preferred composite material for retrofit application is Fiber Reinforced Polymers (FRPs), which consists of high strength glass, carbon, or aramid fibers bound in an epoxy resin matrix. Recent developments in the manufacturing of fiber reinforced polymer (FRP) composite materials have made these materials available for a wide range of applications, including seismic retrofit of 
reinforced concrete columns. Compared to steel and concrete jacketing, FRP wrapping has several advantages, including very low weight-to-strength ratios, high elastic moduli, resistance to corrosion, and ease of application. FRP can improve column ductility without considerable stiffness amplification while maintaining the bridge dynamic properties (Haroun and Elsanadedy 2005). As mentioned before, carbon fiber reinforced polymer (CFRP), glass fiber reinforced polymer (GFRP) and aramid fiber reinforced polymer (AFRP) are the most commonly used FRP composite materials. CFRP has a higher modulus of elasticity and has the highest tensile strength. The Washington State Department of Transportation recommends using CFRP to retrofit bridges because it is less affected by moisture than GFRP (WSDOT 2006).

Several analytical and experimental studies involving retrofitting of columns using FRPs can be found in the literature. To name some, Seible et al. (Seible et al. 1995) describes the jacket design aspects, jacket installation, full-scale field tests and behavior performance of carbon jacket retrofits. Xiao et al. (Xiao et al. 1999) reported on testing of three large-scale bridge column tests, in which two were retrofitted using a prefabricated composite wrapping system. The results of both studies showed that composite jacketing was a viable retrofit measure for shear enhancement and ductility improvement. Finally, Teng et al. (Teng et al. 2001) provides an excellent overview of the use of advanced composites in the seismic retrofit of concrete structures and its use in Civil Engineering.

FRP retrofit systems can be effectively used for circular columns since circular jackets provide a continuous confinement pressure. On the contrary, for rectangular columns the FRP jacket provides less confinement pressure since its confinement effectiveness is concentrated in the corners only. However, recent studies at Portland State University (Mehary et al. 2014) showed that seismically deficient square reinforced concrete columns can be effectively retrofitted using Carbon Fiber Reinforced Polymer (CFRP). The objective of that study was to mitigate the damage effect that an increasing number of inelastic cycles imposed by subduction zone mega earthquakes has in RC columns. The CFRP composite material called Tyfo ${ }^{\circledR S C H}-41$ was used for retrofitting the square RC column. Three layers of this composite material was utilized to wrap the base of the column up to 36 inches high in an effort to improve the confinement of the column and prevent lap splice failure at a potential plastic hinge location as shown in Figure 5.9(a). It is worth noting, that the column corners were chamfered prior the installation of the FRP wrap in order to avoid stress concentrations at the corners and improve the effect of the FRP confinement. The results of the experimental study demonstrated that the CFRP retrofit measure considerably enhanced the displacement ductility of the column without greatly increasing column strength as depicted in Figure 5.9(b). This result is relevant in the evaluation of the other bridge components since an increase in column strength would result in an increased force transfer to cap beams and footings. The retrofit also helped inhibit bond failures in lap splices and provided passive confinement producing very minimal spalling of concrete at the end of the test. 

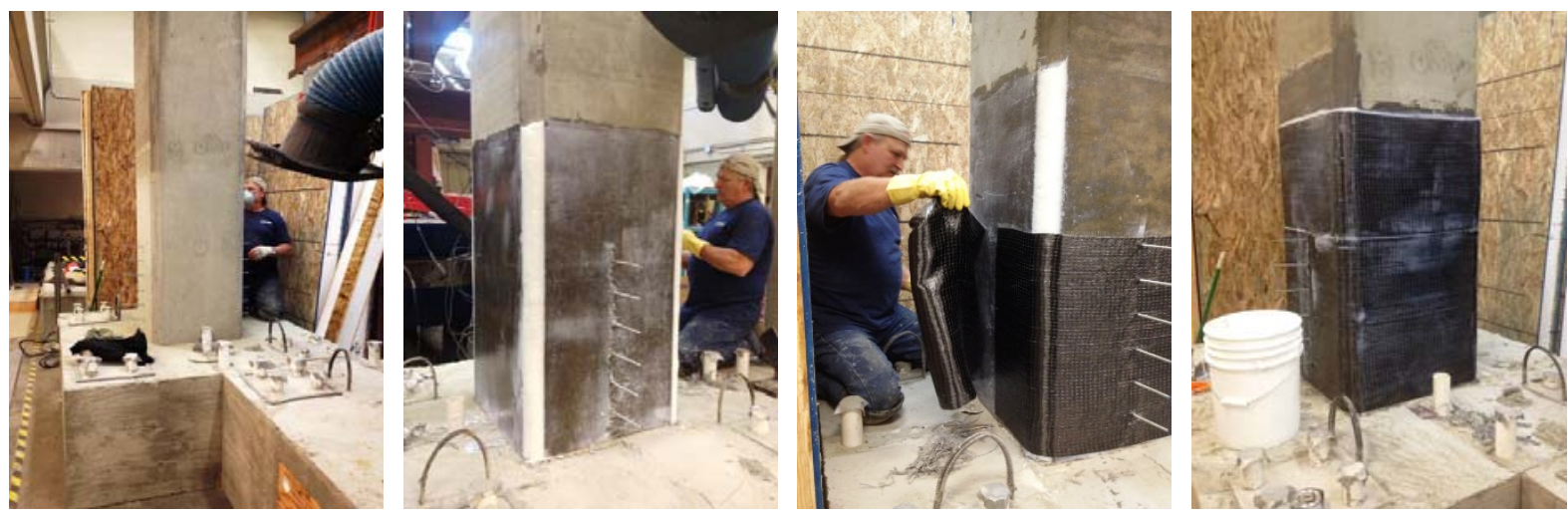

(a)

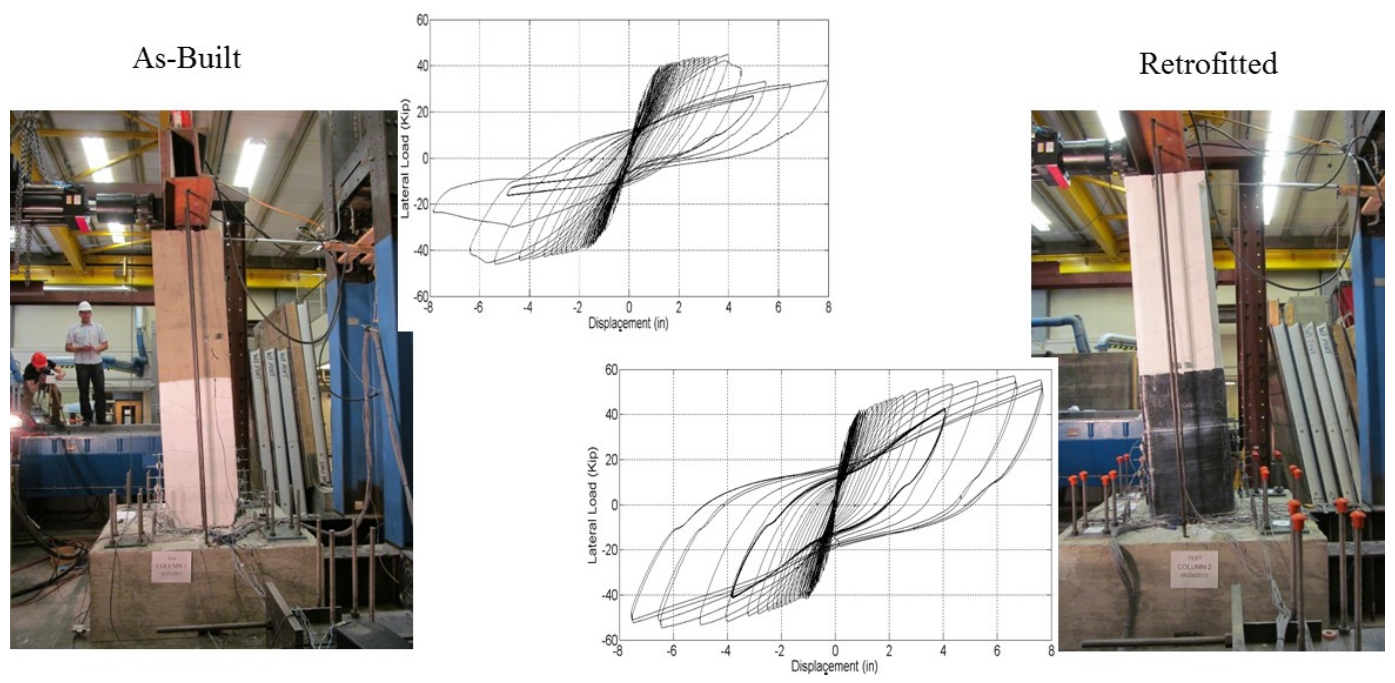

(b)

Figure 5.9: Composite jacketing of a deficient square RC column. (a) Installation of CFRP, (b) Results (Mehary et al. 2014)

\subsubsection{External Prestressing Steel}

Different that the retrofitting systems presented before, which rely in passive confinement, the external prestressing steel acts by providing external confining forces into the column. In this retrofit measure, external prestressing steel wires under tension are wrapped around a column to achieve confinement. This retrofit method has successfully increased the flexural ductility of circular columns with lap splices at the critical section, but its effect on shear strength has not yet been quantified (FHWA 2006).

An advantage of this practice is that it does not affect the flexural strength and stiffness of the columns. However, the high cost of designing a machine that is big enough to produce the required tension to wrap prestressing wire around the columns makes it uneconomical. 
Saatcioglu and Yalcin (Saatcioglu and Yalcin 2003) applied external prestressing to columns in transverse direction using individual hoops that consist of prestressing strands and specially designed anchors. They tested the columns under constant axial compression and incrementally increasing lateral deformation reversals. Their results show that this retrofit methodology can mitigate shear failure, increase flexural capacity and inelastic column deformability.

Recent development in material sciences has allowed engineers the use of new materials for the retrofitting of RC columns. For example, shape memory alloys have been recently studied to achieve an active confinement for the retrofitting of RC bridge columns (Andrawes et al. 2010).

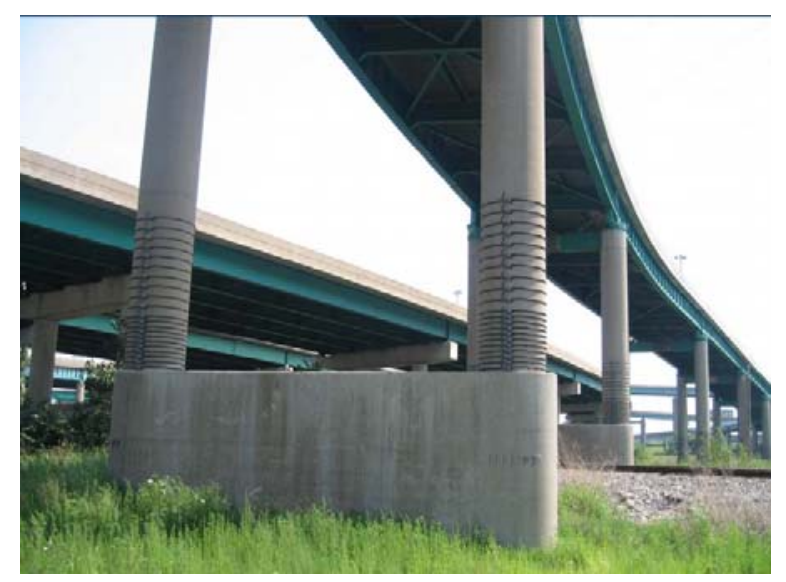

Figure 5.10: External prestressing steel retrofitting of columns in Illinois

\subsection{RETROFIT MEASURES FOR RC BRIDGE BENTS}

Although the retrofit measures discussed in the previous section are also applicable for $\mathrm{RC}$ bridge bents, this section presents retrofit alternatives that aim to improve the overall behavior of $\mathrm{RC}$ bents more than just improving a specific component.

\subsubsection{Infill Walls}

Adding shear walls or braced systems in RC structures has been commonly used as strengthening method. Figure 5.11 illustrates different methods used for retrofitting of RC frames in buildings. Steel braced frames and post-cast wall, also referred to as infill shear wall as shown in Figure 5.12 (FHWA 2006), are the most efficient retrofit measures in terms of enhancing transverse shear capacity. However, it is clear that the steel framed brace is more effective since it also enhances displacement capacity. Despite this fact, infill walls have been commonly applied to retrofit multi-column $\mathrm{RC}$ bridge bents due to their lower cost and familiar implementation in the field.

Moreover, Pulido et al. (Pulido et al. 2004) performed shake table testing of a $1 / 4$ scale as-built bent representing typical bridge bents constructed before the 1970s and an identical specimen retrofitted with an infill wall. The results of this study showed that the use of infill walls can enhance the seismic performance of the as-built bent by increasing both the strength of the bent and its ductility. 


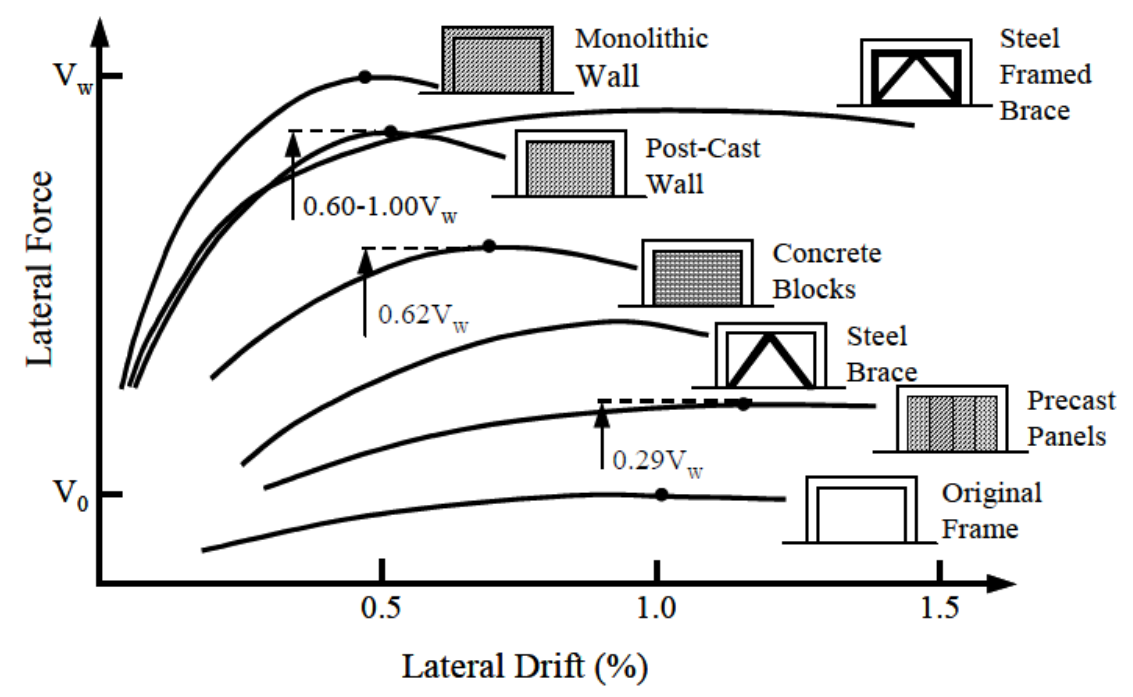

Figure 5.11: Effect of different retrofit measure for RC frames. (Sugano 1981)

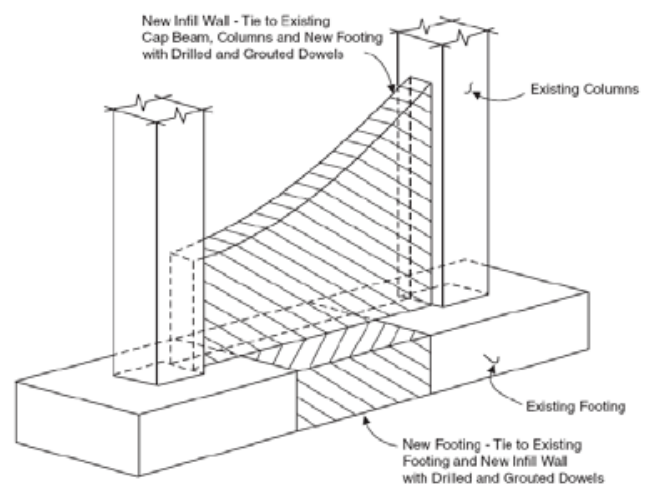

(a)

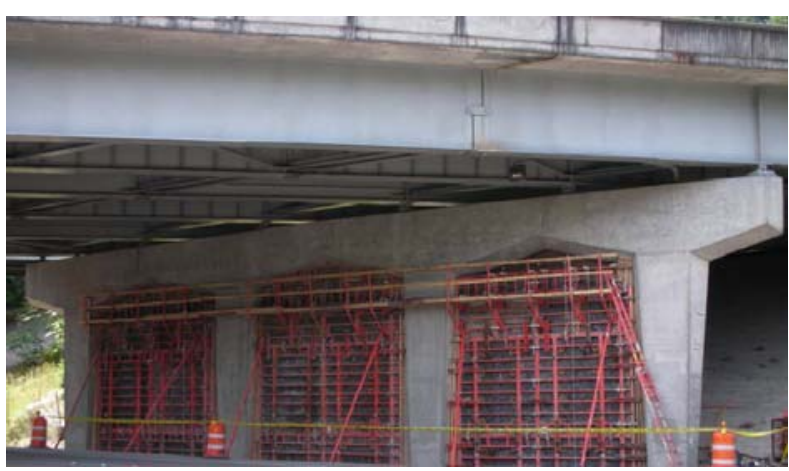

(b)

Figure 5.12: Infill wall, (a) schematic, (b) Installation on West Lake Sammish Parkway (photo: L.M. Marsh)

\subsubsection{Steel Bracing}

Several analytical and experimental studies have been conducted on the use of steel bracing as a seismic retrofit technique for RC frame buildings (Badoux and Jirsa 1990), (Yamamoto and Umemura 1992). However, its implementation in bridges has been limited to steel diaphragms and steel bridges (Itani 1996).

Current research into seismic retrofitting with steel bracing mostly involves adding supplemental damping devices in an effort to minimize the increase in strength that the retrofit measure would impart to the rest of the components of the structure (McDaniel 2006). Among the damping devices that have been proposed for seismic retrofitting of structures can be found friction dampers, viscoelastic dampers and hysteretic dampers. 
This study, as addressed in the next chapters, presents the design implementation and experimental results of retrofitting deficient $\mathrm{RC}$ bridge bents by adding steel bracing in form of hysteretic dampers. 


\subsection{STRUCTURAL FUSES AS SEISMIC RETROFITTING MEASURE FOR MULTI-COLUMN RC BRIDGE BENTS}

\subsection{GENERAL}

Current seismic retrofitting manuals and guidelines for existing highway structures in the United States are based on a performance-based design methodology, which uses a multiple-level approach to performance criteria with two seismic hazard levels (FHWA 2006). In this methodology, damage states can be presented in terms of physical damage and strain limits or curvature limits. Therefore, engineers designing retrofit measures for improving the seismic resistance of standard existing bridge substructures need to ensure that the structure remains operational under a moderate earthquake and that life safety is preserved after a large earthquake. Seismic retrofit measures including steel jacketing and fiber composite wrapping have been typically utilized to improve the ductility and shear resistance of reinforced concrete substructures. However, these measures neither prevent damaging of columns nor excessive displacements, which in some cases could result in not meeting the intended performance level. In order to overcome this problem, this chapter discusses the option of using sacrificial elements also referred to as structural fuses as a retrofit measure for reinforced concrete bridges.

\subsection{ACTUAL SEISMIC RETROFIT DESIGN PHILOSOPHY}

After the devastating earthquakes in San Francisco, CA in 1971 and Loma Prieta, CA in 1989, the American Association of State Highway and Transportation Officials (AASHTO) adopted a forced-based design methodology, which were based largely on design criteria developed by the California Department of Transportation (Caltrans). Over the years, it became evident that this methodology did not provide a rational assessment of displacement demand/capacity needed for bridge structures. Consequently, AASTHO modified this methodology for a displacement-based criteria (AASHTO 2009). This new methodology uses a design earthquake with 7 percent probability of exceedance in 75 years (Approx. 1000-year return period) and four seismic design categories (SDC). Under the AASHTO code requirements bridges are primarily designed to meet a standard performance level based on a life-safety approach, which means the bridge has a very low probability of collapse when subjected to earthquakes that are most likely to occur over the life of the structure. Nowadays, the seismic design philosophy of bridges is trending to a performance-based design, in which different performance levels need to be satisfied under representative seismic hazards. For seismic retrofit of bridges the Federal Highway Administration (FHWA) released a performance-based guideline in 2006, which uses a multilevel performance criteria. Following the FHWA steps a few Departments of Transportation (DOTs) have adopted seismic design performance criteria in their manuals and regulations. This is the case for the Oregon Department of Transportation (ODOT), which for both new and existing bridges adopted a two-level performance criteria (ODOT 2014). The two-level performance criteria for existing bridges are "Life Safety" under a 1000-year event and "Operational" under a 500-year event, following the guidelines described in the FHWA Retrofitting Manual (2006). For new bridges ODOT requires the same dual performance levels 
but some modifications to the design requirements were made depending on the Seismic Design Category (SDC). The inclusion of this additional lower level performance criteria ("Operational") is above the standard performance requirements prescribed by AASHTO.

\subsection{STRUCTURAL FUSE RETROFIT STRATEGY}

The recent occurrence of highly devastating earthquakes near instrumented regions, e.g. 2010 Maule, Chile and 2011 Tohoku, Japan, has again shown the vulnerability of RC bridges subjected to subduction zone mega earthquakes. Nowadays, extensive research on structural damage control concepts has taken place in order to develop more effective and reliable retrofit strategies for existing structures subjected to strong ground motions. One alternative that has attracted the focus of current investigations is the use of structural fuses. This structural fuse retrofit concept consists of integrating replaceable components in the main structural system in such a way to restrict the damage undergoing for the primary structure after a damaging earthquake (Connor et al. 1997). The replaceable elements are designed to take the earthquakeinduced energy and dissipate it through nonlinear hysteretic behavior, meanwhile, the remaining structure is expected to behave elastically. This study discusses the inclusion of steel braced systems such as Buckling Restrained Braces (BRBs) and Eccentrically Steel Braced Frames (EBF) as structural fuses for the retrofitting of seismically deficient RC bridge bents. The motivation of this retrofit strategy is basically to help overcome the imminent risk of extensive damage in deficient bridge bents constructed in the 1950 to mid-1970 in the Pacific Northwest, and consequently to satisfy the aforementioned performance criteria for deficient existing RC bridges.

\subsection{STRUCTURAL FUSES FOR BRIDGES}

The retrofit strategy adopted here is focused in the transverse direction and is based on employing two independent structural systems. The reinforced concrete multi-column bent, which is the primary system and supports the vertical loading; and a replaceable system that add lateral stiffness and functions as the seismic energy dissipation device. For the retrofitted bridge bent system, the fuse system could be either a Buckling Restrained Brace System (BRB) or an Eccentrically Braced Frame System (EBF), which are designed to dissipate energy through nonlinear hysteretic behavior; meanwhile, the $\mathrm{RC}$ bent and connections are designed to remain elastic. This structural concept has potential advantages over conventional retrofit systems. Some of them are:

- Since the gravity resisting system is designed to remain elastic after a major earthquake event, higher performance levels can be achieved for the 500 -year and 1000 -year events.

- Energy dissipation through stable nonlinear hysteretic behavior.

- The stiffness of the system is increased providing a drift-controlled system

- Ideally, the sacrificial elements can be easily replaced, minimizing the repair time and allowing uninterrupted service in the bridge 
- By forcing the inelastic demand to the fuses, the behavior of the system becomes more predictable.

The system is not exempt of disadvantages and challenges. For example, special assessment of elements capacities needs to be performed since the forces in the system may increase due to the added strength and stiffness of the replaceable element. Moreover, the connection design between steel and concrete components also need special attention.

In the longitudinal direction, depending on the type of abutment, the bridge can engage the abutments that significantly influence the deformation demand. This is the case for bridges with integral and semi-integral abutments where the passive resistance of the soil against the backwall increases the seismic force transferred to the abutment backfill, and decreases the seismic demands on the bridge bents. While the longitudinal direction is important to consider, procedural details have not been included in this study.

\subsubsection{Buckling Restrained Brace systems (BRB)}

Buckling restrained braces were introduce in the US in the late 1990's and several experimental tests and post-earthquake reconnaissance in multi-story framed buildings have shown that these structures may be efficiently retrofitted using BRBs. The main characteristic of a BRB is its ability to have a stable hysteretic response through yielding both in tension and compression due to the prevention of global buckling as shown in Figure 6.1 (Clark et al. 1999). This inherent property of providing higher hysteretic energy dissipation makes BRBs a good candidate in seismic retrofit applications, where the main structure can be designed to respond elastically and the braces become disposable elements capable of absorbing and dissipating the earthquakeinduced energy. As with any metallic dissipation device, BRB behavior depends on its geometry and mechanical characteristics and its effectiveness depends on its energy dissipation capacity. A typical BRB anatomy is illustrated in Figure 6.2.

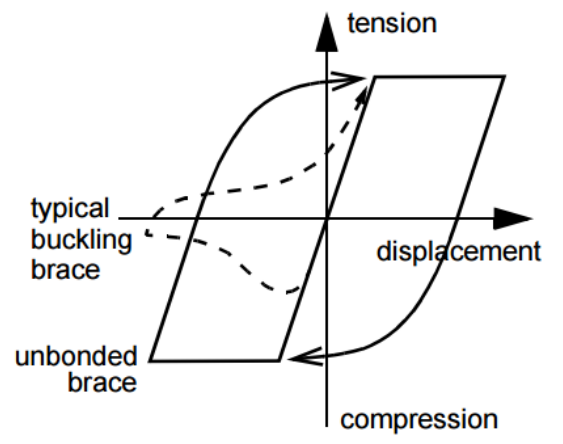

Axial force-displacement behavior

Figure 6.1: Hysteretic behavior of a BRB. (Clark et al. 1999) 


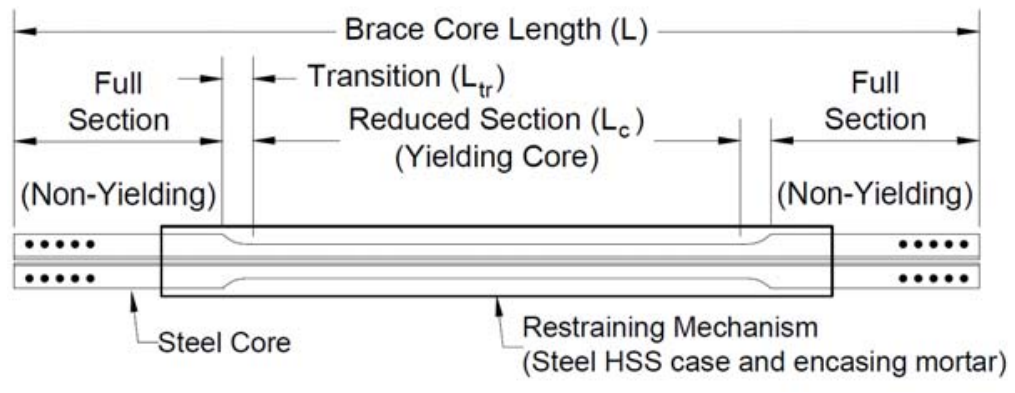

Figure 6.2: Schematic of a Buckling Restrained Brace (BRB)

Despite the increasing use of this system for buildings, its use has not been realized for bridge structures. Few BRB applications in bridges can be found, e.g. feasibility of using BRBs for the Vincent Thomas Bridge in Long Beach, California (Lanning et al. 2011) and seismic retrofit of California's Auburn-Foresthill Road Bridge (Reno and Pohll 2010). However, none has been implemented for seismic retrofitting of common reinforced concrete multi-column RC bridge bents. The objective of this research is to advance the seismic retrofit of reinforced concrete bridges subjected to moderate and large earthquakes by using buckling restrained braces (BRBs) as a retrofit measure. For the retrofitted bridge bent system, the BRB is designated as the "fuse or replaceable" element and the RC bent is designated as the primary system as shown in Figure 6.3. The proposed configuration consists of a single diagonal brace (Figure 6.3(b)) but its use is not limited to that configuration as shown in Figure 6.3(c). This approach has the potential to improve the overall seismic behavior and the expected performance levels especially under performance driven design criteria.

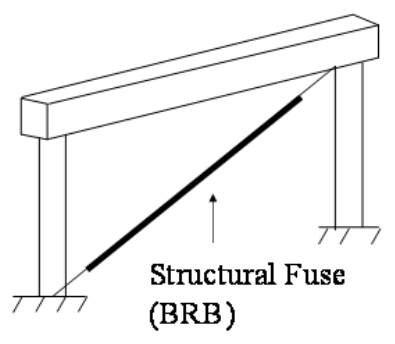

(a)

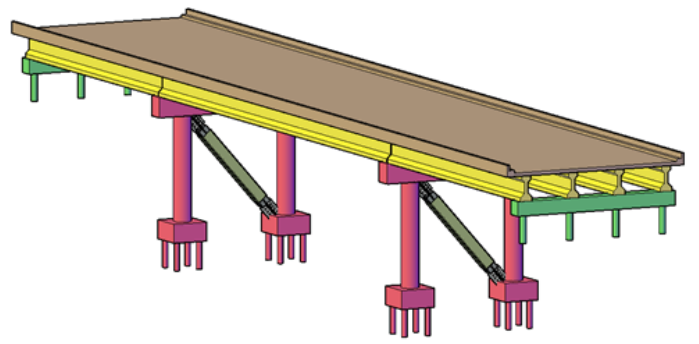

(b)

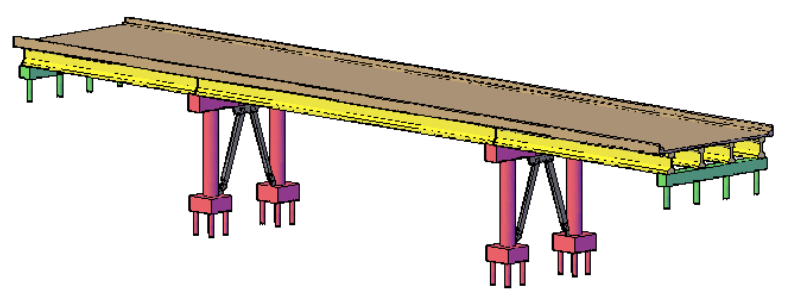

(c)

Figure 6.3: BRB retrofit configuration. (a) Structural fuse schematic. (b) Diagonal configuration. (c) Chevron configuration 


\subsubsection{Eccentrically Braced Frames (EBF)}

Eccentrically Braced Frames are a lateral load resisting system where the inelastic behavior is concentrated in a specific member referred to as "link". During a major seismic event the link is subjected to inelastic deformations in shear and flexure, meanwhile the other components remain essentially elastic. The link demands in shear and flexure are generated by the eccentricity formed between the intersection of the centerlines of adjacent beam, brace and columns as shown in Figure 6.4. For the structural fuse concept the link is designated as the structural fuse element.
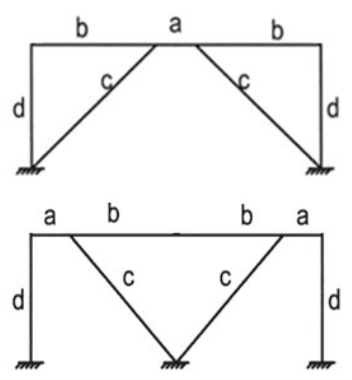
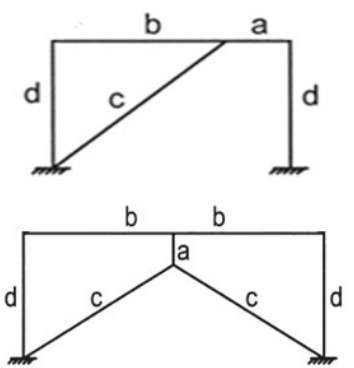

$a=$ link

$b=$ beam segment outside of link

$c=$ diagonal brace

$d=$ column

Figure 6.4: Different EBFs configurations. (AISC 2010)

EBFs as well as BRB present a stable hysteretic response, which may be translated to higher hysteretic energy dissipation, making EBFs and BRBs good candidates in seismic retrofit applications. The hysteretic behavior of an EBF system is depicted in Figure 6.5 (Manheim 1982). Eccentrically Braced Frames gained considerable attention and numerous analytical and experimental studies were carried in the 1980's. An excellent introduction and overview of the behavior of eccentrically braced frames can be found in Popov and Engelhardt (Popov and Engelhardt 1988).

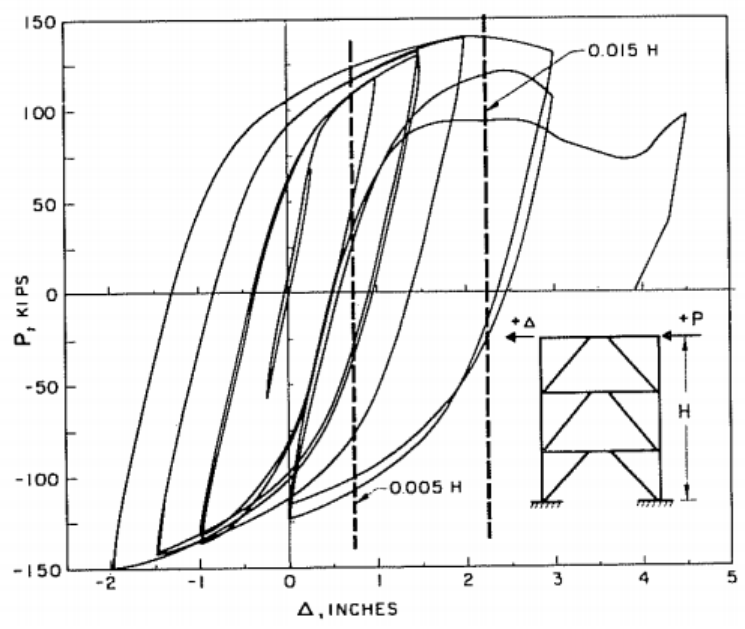

Figure 6.5: Hysteretic behavior of EBF system. (Manheim 1982)

This type of retrofit measure has not been implemented in bridge substructures since its application has been limited to bridge decks and buildings. Furthermore, the implementation for retrofitting RC structures found in the literature is limited to analytical and experimental studies 
(Bouadi 1994), (Ghobarah and Elfath 2001). Among the scarce experimental studies involving EBF as a retrofit measure for RC structures, Mazzolani (Mazzolani 2009) carried out the testing of three EBF with vertical shear links as shown in Figure 6.8. Mazzolani concluded that a stable behavior and excellent ductility of the whole system can be achieved by the inclusion of eccentrically brace frames.
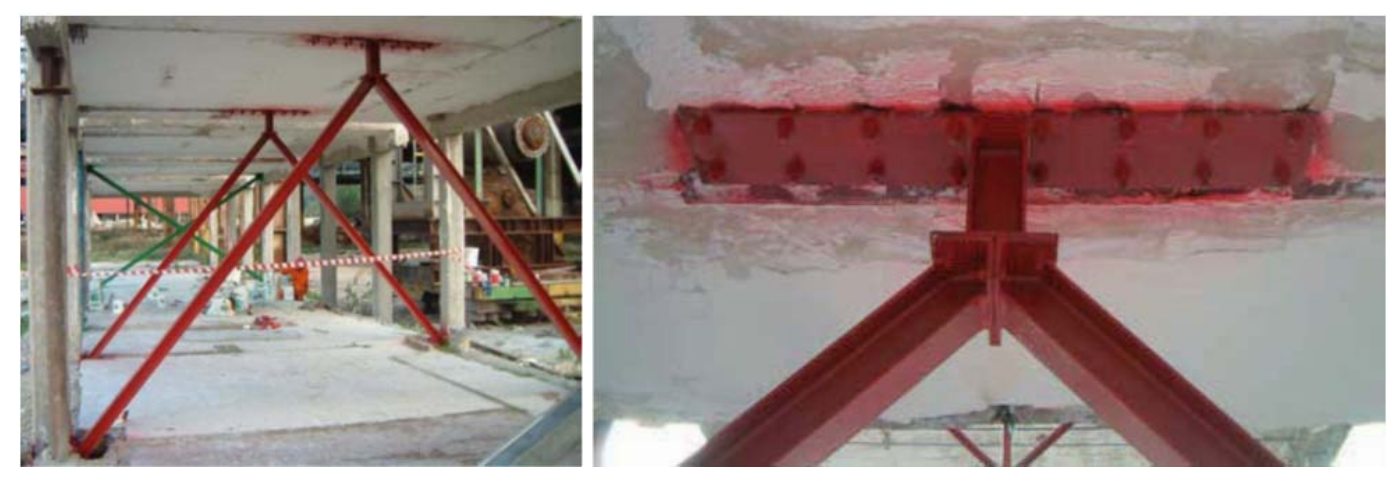

Figure 6.6: Experimental study of using EBF system to retrofit RC structures. (Mazzolani 2009)

\subsection{DESIGN IMPLEMENTATION OF STRUCTURAL FUSES}

\subsubsection{General Design Implementation}

As in any design, in order to retrofit deficient RC multi-columns bridge bents by using structural fuses is necessary to iterate until all the limit states of each component are verified and a proper design is achieved. For this purpose a retrofit procedure needs to follow at least 4 primary steps as shown in Figure 6.7 and described below.

1. Assessment of the as-built RC bent. The capacity of the bare bent and the demand obtained from two seismic hazards (500 and 1000-year events) need to be assessed

2. Sacrificial element design following the structural fuse concept. Select location and configuration. Determine stiffness and member dimensions.

3. Connection Design. The connection between sacrificial elements to gusset plate and gusset plate to concrete components need to be performed.

4. Column, cap beam and footing capacity check is needed to ensure that the primary system remains elastic or with minor damage after the dual seismic demand. 


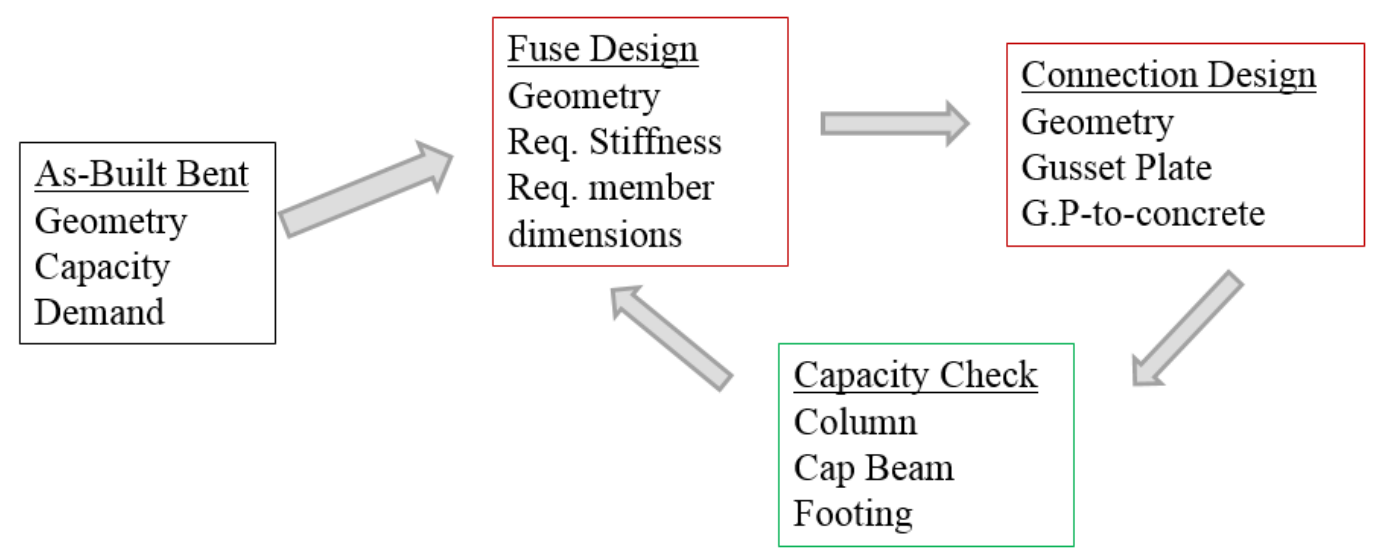

Figure 6.7: Structural fuse design implementation

\subsubsection{Assessment of the as-built RC bent}

In this step, the capacity of the bare bent and the demand obtained from two seismic hazards (500 and 1000-year events) need to be assessed.

The as-built RC multi-column bridge bent capacity and demand need to be assessed. A general pushover analysis is required to determine the capacity curve of the RC bent as shown in Figure 6.8.

For the general pushover is required to use the material properties from actual testing or by assuming expected properties. Expected material properties can be determined by applying factors to the specified properties. Factors of 1.3 and 1.1 are recommended in the literature for the compressive strength of concrete and the yield stress of steel, respectively. It is worth to mention that a general pushover analysis is suitable in this case since the first mode usually controls the behavior of RC bridge bents in both the transverse and longitudinal directions.

The demand is obtained from subjecting the bare bent to two seismic hazards, which are a 500 -year event (14 percent probability of exceedance in 75 years) and a 1000-year event (7 percent probability of exceedance in 75 years). An elastic analysis of an equivalent single degree of freedom system by using the response spectrum from each event and applying a displacement magnification $\left(\mathrm{R}_{\mathrm{d}}\right)$ for short-period structures as per AASHTO (AASHTO 2009) is required as follows:

$$
\begin{gathered}
\delta_{(500,1000)}=R_{d} \frac{S_{a,(500,1000)} T_{e}^{2} g}{4 \pi^{2}}=R_{d} S_{a} g \frac{m}{K_{S}} \\
R_{d}=\left\{\begin{array}{c}
\left(1-\frac{1}{\mu_{D}}\right) \frac{1.25 T_{s}}{T_{e}}+\frac{1}{\mu_{D}} \geq 1.0 \text { for } \frac{1.25 T_{s}}{T_{e}}>1.0 \\
1.0 \text { for } \frac{1.25 T_{s}}{T_{e}} \leq 1.0
\end{array}\right.
\end{gathered}
$$


Where, $T_{e}$ is the fundamental period of the SDOF system; $S_{a}$ is the spectral acceleration given by the respective response spectrum; $\mathrm{g}$ is the standard gravity constant; $\mathrm{m}$ is the inertial mass of the system, $K_{\mathrm{s}}$ is the stiffness of the system, $\mathrm{T}_{\mathrm{s}}$ is the period at the end of constant design spectral acceleration plateau and $\mu_{\mathrm{D}}$ is the maximum local member displacement ductility demand. AASHTO states that $\mu_{\mathrm{D}}$ may be taken as 6 .

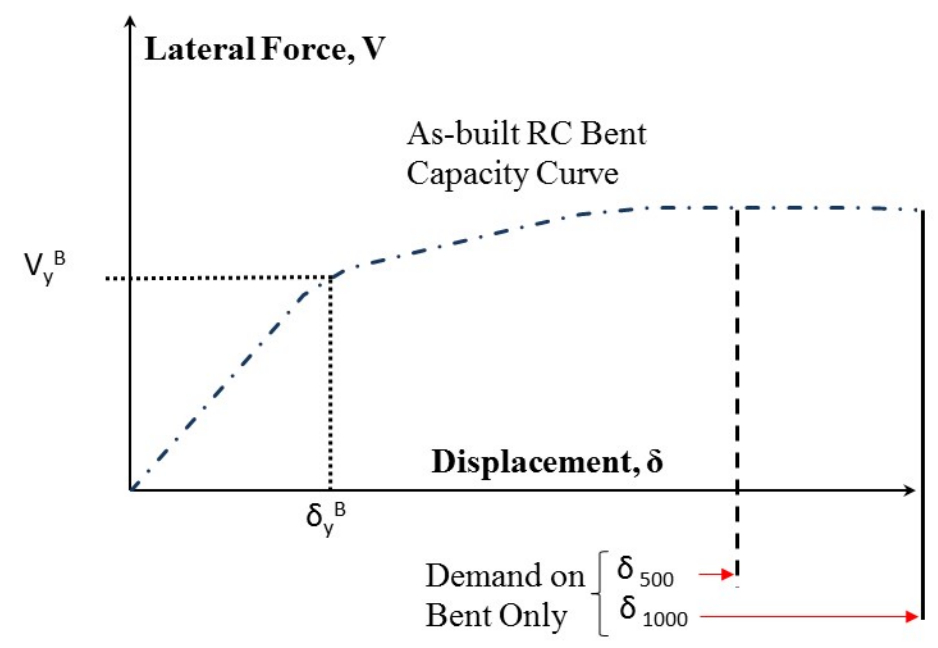

Figure 6.8: Assessment of as-built RC bent

\subsubsection{Structural Fuse Concept Design}

The structural fuse concept design procedure is similar to the one proposed by (Vargas and Bruneau 2009) for buildings and El-Bahey and Bruneau (El-Bahey and Bruneau 2011) for bridges but with some modifications. The design concept, as already stated, needs that the primary structural system remains essentially elastic after a major earthquake. In order to fulfill this design concept and satisfy the performance criteria for existing bridges described in the FHWA (FHWA 2006) guideline, the fuse component needs to be designed in such way to reduce the demands on the bare frame, which ideally would respond elastically under the 500-year and 1000-year event. However, due to the increased forces in the system, in some cases the demands in the concrete elements exceed their capacity making the design of the fuse infeasible. For that reason, minor inelastic excursions are allowed to occur under the 1000-year event as shown in Figure 6.9. A maximum displacement of 140 percent the yield displacement is recommended. This displacement is recommended in an effort to limit the damage and still provide for a system ductile response.

For the fuse behavior is assumed a bi-linear response with hardening as depicted in Figure 6.9. The strength hardening effect is important to represent since it increases the demands in the concrete elements. Important parameters in the fuse contribution are the yield displacement of the fuse $\left(\delta_{\mathrm{y}}^{\text {Fuse }}\right)$, the lateral force at that displacement $\left(\mathrm{V}_{\mathrm{y}}^{\text {Fuse }}\right)$, the initial stiffness $\left(\mathrm{k}_{\mathrm{i}}^{\text {Fuse }}\right)$ and any over-strength factor affecting the hardening of the fuse. 


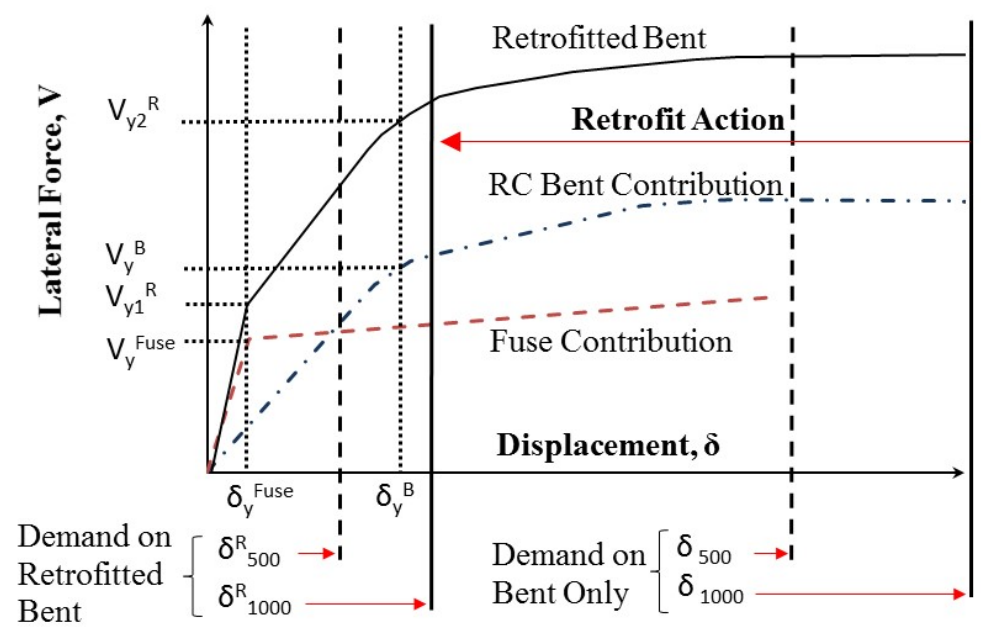

Figure 6.9: Idealized structural fuse retrofit design

The first step to design the structural fuses is to set the following parameters:

- Material properties $\left(\mathrm{f}_{\mathrm{y}}, \mathrm{E}_{\mathrm{s}}\right)$ that are limited to commercial products

- Configuration of the fuse system $\left(\mathrm{L}_{\mathrm{b}}, \theta\right)$ that is limited by the existing bridge dimensions.

The following step, as first iteration, is to set the displacement demand from the 1000year event equal to or less than 1.4 times the yield displacement of the bare frame $\left(\delta_{\mathrm{y}}{ }^{\mathrm{B}}\right)$ extracted from the assessment of the as-built RC bent as shown in Eq. (6.2).

$$
\delta_{(\text {demand })}^{R}=R_{d} S_{a} g \frac{m}{K_{S}} \leq 1.4 \delta_{y}{ }^{B}
$$

The system composed by the $\mathrm{RC}$ bent and the structural fuse can be analyzed as a system in parallel. In such configuration, the displacement in global coordinates $(\delta)$ is the same for both the RC bent and the structural fuse. The strength (V) and stiffness (K) of each element is added to obtain the system strength and stiffness as follows:

$$
\begin{aligned}
& V^{R}=V^{B}+V^{\text {Fuse }} \\
& K_{s}=k_{i}^{B}+k_{i}^{\text {Fuse }}
\end{aligned}
$$

The initial stiffness of the as-built $\mathrm{RC}$ bent $\left(\mathrm{k}_{\mathrm{i}}^{\mathrm{B}}\right)$ can be directly determined from the pushover curve or by using an elastic analysis assuming crack sections as shown in Eq. (6.5). 


$$
\begin{array}{r}
k_{i}^{B}=\frac{24 E_{c c} I_{c, c r a c k}}{H^{3}} \cdot \frac{12 \rho+1}{12 \rho+4} \\
\rho=\frac{E_{c b} I_{b}}{L} \cdot \frac{H}{2 E_{c c} I_{c}}
\end{array}
$$

Where, $\mathrm{E}_{\mathrm{cc}}$ and $\mathrm{E}_{\mathrm{cb}}$ are the modulus of elasticity of concrete in the column and beam respectively; $\mathrm{I}_{c, \text { crack }}$ is the cracked inertia of the column; $\mathrm{I}_{\mathrm{b}}$ and $\mathrm{I}_{\mathrm{c}}$ are the inertia of the beam and column respectively; $H$ is the height of the bent; $L$ is the span of the bent and $\rho$ is a modification factor that take into account that the cap beam is not infinitely rigid.

Since the demand depends on the fundamental period of the system, the structural fuse design, as discussed in this paper, is reduced to iterate until the stiffness of the fuse system is determined. Consequently, the steel core area and the reduced length section $\left(\mathrm{L}_{\mathrm{c}}\right)$ of the BRB are obtained as follows:

$$
k_{i}^{\text {Fuse }} \geq \frac{R_{d} S_{a} m g}{1.4 \delta_{y}{ }^{B}}-k_{i}{ }^{\mathrm{B}}
$$

The final implementation and design is recommended to be in coordination with the fuse manufacturer. This is because some of the parameters needed for the structural fuse design are dependent on the design of the fuse itself and may differ from manufacturer to manufacturer.

\subsubsection{Connection Design}

Any steel to gusset plate connection shall be designed to resist the demands based on adjusted strengths in an effort to satisfy capacity design concepts as per AISC (2010).

$$
P_{a d}=\Omega R_{y} P_{y}
$$

Where, $\mathrm{P}_{\mathrm{ad}}$ is the adjusted strength; $\Omega$ is an over-strength factor; $\mathrm{R}_{\mathrm{y}}$ is the ratio of the expected yield stress to the specified minimum yield stress (usually taken as 1.1) and $\mathrm{P}_{\mathrm{y}}$ is the yield strength of the connecting member. Over-strength factors shall be obtained from a qualification test or provided by the manufacturer.

The limit states that need to be checked for the design of the gusset plate according to AASHTO (AASHTO 2009) and AISC steel manual (AISC 2011) are: tensile yielding of Whitmore section, tensile rupture of Whitmore section, block shear in tension, buckling in compression, yield moment strength, plastic moment strength, weld/bolts for gusset to brace connection, and buckling of unsupported edge. For bolted connections is recommended the use of slip critical connections.

Anchor rods are recommended for connecting the gusset plate with concrete elements. The design forces are obtained from the adjusted strength and the correspondent gusset plate free body diagram. According ACI318 Appendix D (ACI318 2011), tensile and 
shear strength must be considered in the connection design. For tensile strength, five possible failure modes can appear in this load direction, namely, steel failure of anchor in tension, concrete breakout, pullout, concrete side-face blowout, and concrete splitting. For shear strength, three failure modes are considered, namely, steel failure in shear, concrete breakout and concrete pryout. The failure modes in tension and shear mainly depend on steel grades, bolt spacing, concrete cover and bolt embedment. Additionally, for anchors that are subjected to both shear and tension a shear-tension interaction shall be satisfied.

\subsubsection{Capacity Check}

Due to the incorporation of structural fuses, the internal forces in the concrete elements may increase considerably. This is particularly important when an oversized steel member is used. For that reason, a capacity check of the RC elements is crucial for the success of the design concept. The capacity of concrete elements can be computed by using the requirements in AASHTO (AASHTO 2009) and need to be compared to the internal forces generated from the adjusted load. If the strength requirement of any concrete element is not satisfied, a new structural fuse design iteration is required. Moreover, at the ultimate damage state the formation of plastic hinges has to be verified in order to avoid any undesirable mechanism of collapse.

\subsubsection{Implementation Using BRBs}

For the BRB implementation a bi-linear response with hardening is utilized as illustrated in Figure 6.10. The BRB parameters are the steel core area $\left(\mathrm{A}_{\mathrm{sc}}\right)$, the steel core yield strength $\left(\mathrm{f}_{\mathrm{y}}\right)$, the initial stiffness $\left(\mathrm{k}_{\mathrm{i}}^{\mathrm{BRB}}\right)$ that as first approximation can be assumed as the stiffness provided by the reduced section, the over-strength factors in tension $(\omega)$ and compression $(\beta \omega)$, steel core elongation at initial yield $\left(\Delta_{\mathrm{y}}\right)$, the elongation at the design story drift that as a first approximation can be assumed as $7.5 \Delta_{\mathrm{y}}\left(\Delta_{\mathrm{BM}}\right)$, the axial yield strength of steel core $\left(\mathrm{P}_{\mathrm{ysc}}\right)$ and the adjusted brace strength $\left(\mathrm{P}_{\mathrm{ad}}\right)$.

Additional transformation for the BRB parameters is needed depending on the BRB configuration. The proposed configuration consists of a single diagonal brace. Thus, the aforementioned BRB parameters that are in a local coordinate system can be transformed to a global coordinate system as shown in Figure 6.11 and using equations (6-8) to (6-12).

The relation between the elongation of the $\mathrm{BRB}(\Delta)$ and the lateral displacement of the bent $(\delta)$ is given by Eq. (6.8)

$$
\delta=\frac{\Delta}{\cos (\theta)}
$$

At yield, the stress-strain relationship for the BRB is given by Eq. (6.9). Where, $f_{y}$ is the yield stress, $E_{s}$ is the modulus of elasticity of steel and $\varepsilon_{\mathrm{y}}$ is the strain at yield. Furthermore, the strain at yield is assumed as the strain produced at the reduced section $\left(\mathrm{L}_{\mathrm{c}}\right)$. 


$$
f_{y}=E_{s} \cdot \varepsilon_{y} \rightarrow \Delta_{y}{ }^{B R B}=\frac{f_{y} L_{c}}{E_{s}}
$$

Therefore, the BRB yield displacement $\left(\delta_{\mathrm{y}}{ }^{\mathrm{BRB}}\right)$ is obtained substituting Eq. (6.9) into Eq. (6.8), which yields Eq. (6.10)

$$
\delta_{y}^{B R B}=\frac{f_{y} L_{c}}{E_{s} \cos (\theta)}
$$

Finally, the stiffness and strength contribution to be used in the structural fuse design, as presented in Section 6.5.1.2, is determined by using Equations (6.11) and (6.12).

$$
\begin{gathered}
k_{i}^{B R B}=\frac{E_{s} A_{s c} \cdot \cos (\theta)}{L_{c}} \\
V_{y}^{B R B}=A_{s c} f_{y} \cos (\theta)
\end{gathered}
$$

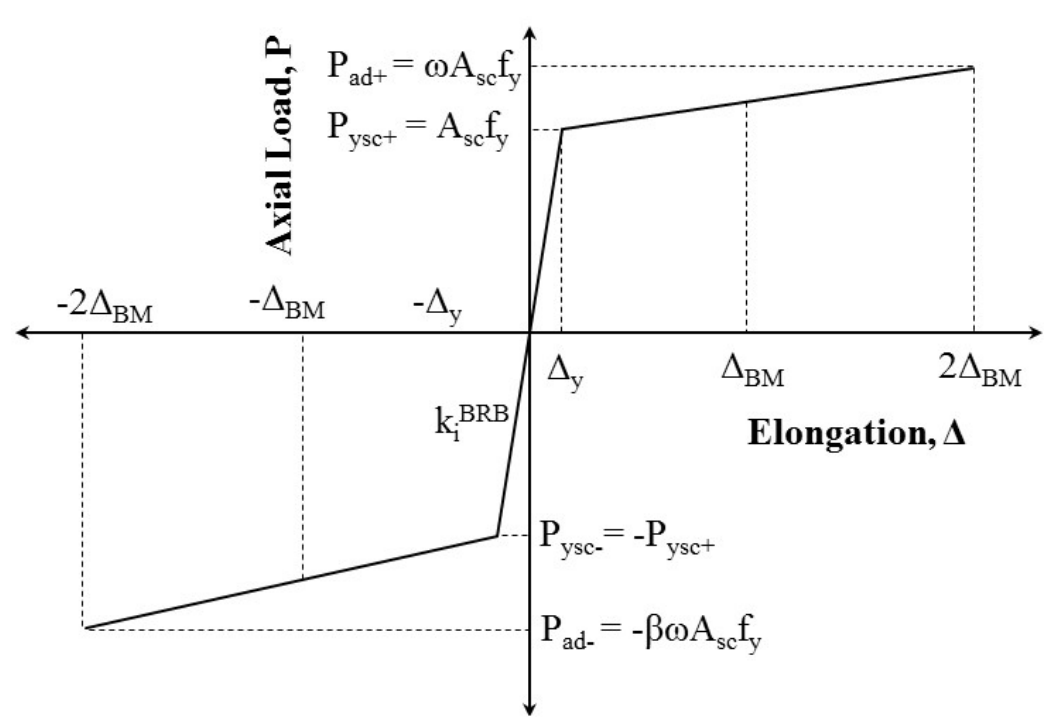

Figure 6.10: BRB idealized behavior 


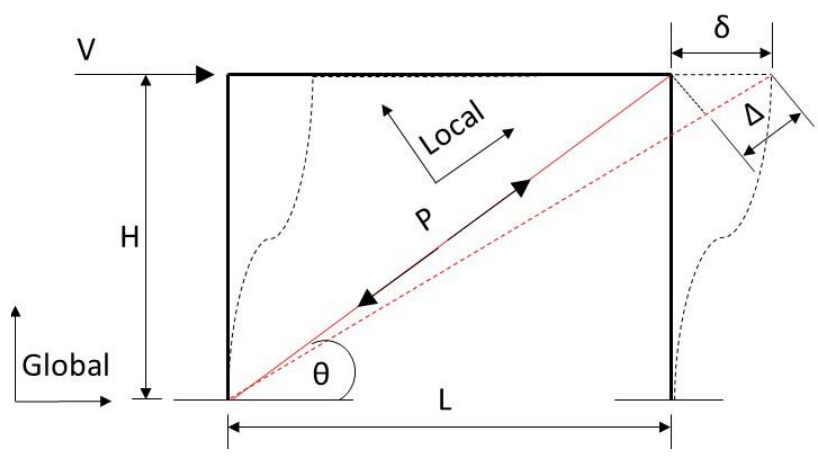

Figure 6.11: BRB parameters trasformation

Since the demand depends on the fundamental period of the system, the BRB design, as discussed in this study, is reduced to iterate until the BRB stiffness is determined. Consequently, the steel core area and the reduced length section $\left(L_{c}\right)$ of the BRB are obtained as follows:

$$
\begin{gathered}
k_{i}^{B R B}=\frac{E_{s} A_{s c} \cdot \cos (\theta)}{L_{c}} \geq \frac{R_{d} S_{a} m g}{1.4 \delta_{y}^{B}}-k_{i}^{B} \\
\frac{A_{s c}}{L_{c}} \geq\left(\frac{R_{d} S_{a} m g}{1.4 \delta_{y}^{B}}-k_{i}^{B}\right) \frac{1}{E_{s} \cos (\theta)}
\end{gathered}
$$

The final implementation and design is recommended to be in coordination with a BRB manufacturer. This is because some of the parameters needed for the BRB design are dependent on the design of the brace itself and may differ from manufacturer to manufacturer. Also, it is worth to mention that in many practical cases the design would result in short reduced sections of the BRB, making not valid the assumption that the BRB stiffness is solely given by the reduced section. Thus, an effective stiffness for the BRB $\left(\mathrm{k}_{\text {eff }}{ }^{\mathrm{BRB}}\right)$ can be used instead as follows:

$$
k_{e f f}^{B R B}=\frac{k_{i}^{B R B} k_{2}^{B R B}}{k_{i}^{B R B}+k_{2}^{B R B}}
$$

Where, $\mathrm{k}_{2}{ }^{\mathrm{BRB}}$ is the stiffness of the non-reduced section of the steel core. This effective stiffness can be provided by the BRB manufacturer.

For the design of connections between BRB to gusset plate, this shall be designed to resist the demands based on the adjusted brace strength as per AISC (AISC 2010).

$$
P_{a d}=\beta \omega \cdot R_{y} \cdot P_{y s c}
$$

Where, $P_{a d}$ is the adjusted brace strength; $\beta \omega$ is the over-strength factor in compression; $R_{y}$ is the ratio of the expected yield stress to the specified minimum yield stress (usually taken as 1.1) and $\mathrm{P}_{\mathrm{ysc}}$ is the axial yield strength of steel core. Over-strength factors shall be obtained from a 
qualification test or provided by the manufacturer. However, an initial assumption of 1.45 for $\beta \omega$ can be used.

\subsubsection{Implementation using EBFs}

In order to design an $\mathrm{EBF}$ as a retrofit measure using the structural fuse concept, the procedure described in Section 6.5.1.2 shall be followed. As a result of that procedure, the initial stiffness and strength of a potential EBF is obtained. The first member to be sized on an EBF is the link as shown in Figure 6.12 for a K-braced EBF and Eq. (6.17). Different EBF configurations are shown in Figure 6.4.
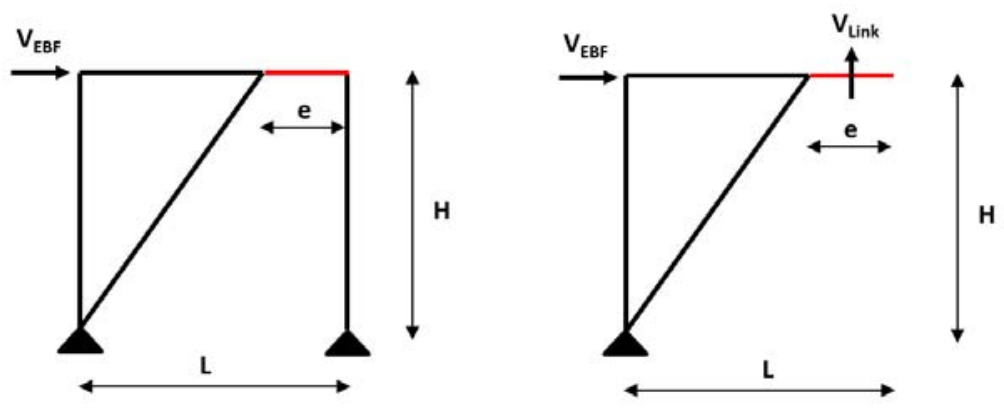

Figure 6.12: Free body diagram for a K-braced EBF

$$
V_{\text {Link }}=\frac{H}{L} V_{E B F}
$$

Where $\mathrm{H}$, is the height of the EBF and $\mathrm{L}$ is the span. Both dimensions are limited by the existing bridge dimensions. Hjelmstad and Popov (Hjelmstad and Popov 1983) carried out studies to assess the seismic behavior of EBF. They found the variation of the elastic lateral stiffness of an $\mathrm{EBF}$ as a function of the link length, e as depicted in Figure 6.13 for two configurations. It is clear that short links provide the maximum possible frame stiffness. However, links that are too short are subjected to excessive inelastic deformation demands.
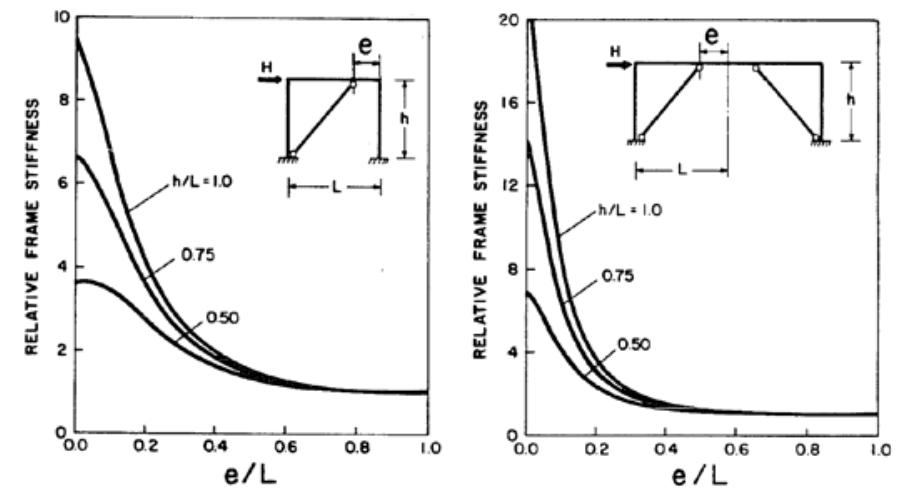

Figure 6.13: Effect of link length, e, in elastic lateral stiffness (Hjelmstad and Popov 1983) 
The design of the EBF system and the other steel members (braces, beams and columns) shall follow the AISC Seismic Provisions (AISC 2010). The use of shear links is recommended in an effort to gain the maximum elastic stiffness from the EBF system.

For shear links the following limitations shall be satisfied as per AISC (AISC 2010):

- Link length, e, is limited by Eq. (6.18) in order to ensure shear yielding in the link:

$$
e \leq 1.6 \frac{M_{p}}{V_{p}}
$$

Where, $\mathrm{M}_{\mathrm{p}}$ is the nominal plastic flexural strength, and $\mathrm{V}_{\mathrm{p}}$ is the nominal shear strength of an active link.

- Maximum link rotation is limited to $0.08 \mathrm{rad}$ in order to assure satisfactory behavior of an EBF in a severe earthquake. The link rotation can be estimated from a rigidplastic mechanism of the EBF as depicted in and Eq. (6.19)

$$
\gamma_{p}=\frac{L}{e} \theta_{p} \leq 0.08 \mathrm{rad}
$$

Where, $\theta_{\mathrm{p}}$ is the plastic story drift angle, $\operatorname{rad}\left(=\Delta_{\mathrm{p}} / \mathrm{H}\right)$.

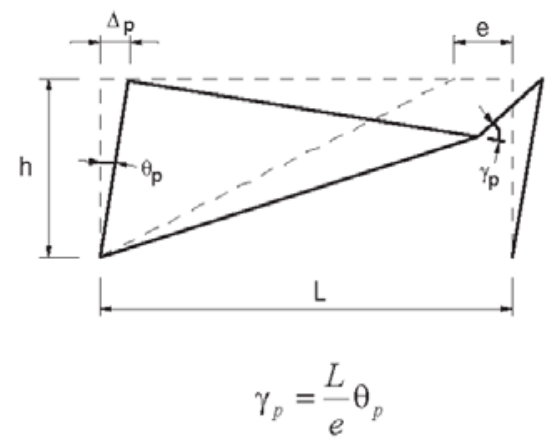

Figure 6.14: Link rotation (AISC 2010) 


\subsection{ASSESSMENT OF RC BRIDGE BENTS UTILIZING BUCKLING RESTRAINED BRACES FOR SEISMIC RETROFIT}

\subsection{GENERAL}

Over the years, earthquakes have exposed the vulnerability of reinforced concrete structures under seismic loads. The recent occurrence of highly devastating subduction zone mega earthquakes near instrumented regions has demonstrated the catastrophic impact of such natural force upon reinforced concrete structures. Typical reinforced concrete bridge bents constructed in the 1950 to mid-1970 in the Pacific Northwest were designed and built with minimum seismic considerations. This resulted in inadequate detailing within plastic hinge zones, leaving numerous RC bridge bents highly susceptible to damage following an earthquake. In order to overcome this deficiency, this chapter presents experimental results of using buckling restrained braces $(\mathrm{BRB})$ as a retrofit measure for multi-column reinforced concrete bridge bents.

Buckling restrained braces were introduce over two decades ago in the US and many experimental tests and post-earthquake reconnaissance in multi-story frame buildings have shown that these structures may be efficiently retrofitted using BRBs. However, the increasing use of this system for buildings has not been reflected in bridge structures.

The main characteristic of a BRB, as presented in the previous chapter, is its ability to have a stable hysteretic response through yielding in both tension and compression due to the prevention of global buckling (Clark et al. 1999). This inherent property of stable hysteretic response, which may be translated to higher hysteretic energy dissipation, makes BRBs a good candidate in seismic retrofit applications. Figure 6.2 illustrates the typical BRB anatomy.

This chapter focuses in the experimental results of seismic performances of a representative halfscale bridge bent retrofitted using buckling restrained braces in a diagonal configuration.

Retrofitted and unretrofitted states were tested under subduction loading protocols in an effort to reflect the displacement demands in $\mathrm{RC}$ bridge bents subjected to subduction zone earthquakes. The braces were designed utilizing the structural fuse concept. The fuse elements, which are the buckling restrained braces, were designed to take the earthquake-induced energy and dissipate it through nonlinear hysteretic behavior; meanwhile, the remaining structure is expected to behave elastically or with minor inelastic excursions.

\subsection{REPRESENTATIVE RC BRIDGE BENT (PROTOTYPE)}

\subsubsection{Selection}

Bridges with prestressed superstructures are the second and third more common type of bridge in the State of Oregon as depicted in Figure 4.1. In an effort to assess a retrofit measure for those bridges, the representative bridge used in this section was extracted from the multi-span prestressed concrete stringer/girder bridge inventory that was built before 1970. The criteria for 
the selection of the representative bridges was inferred from the database found in the 2010 ODOT inventory for prestressed concrete stringer/girder bridges built prior 1970 as follows:

- Selected from 364 Prestressed Concrete Stringer/Girder bridges built prior 1970.

- Number of spans equal to 3 , since 180 out of the 364 bridges has that number of spans.

- Number of lanes equal to 2, since 132 out of the 364 bridges has that number of lanes.

- Representative bridge length ranges from 190 to $210 \mathrm{ft}$.

- Representative bridge width ranges from 30 to $40 \mathrm{ft}$.

- Average superstructure height equal to $31 \mathrm{ft}$.

\subsubsection{Details}

The representative reinforced concrete bent corresponds to an existing RC multi-column bridge bent constructed in the 1950 to mid-1970 in Oregon. As many of the bridge structures built at that time in the Pacific Northwest, the bridge substructure was designed and built with minimum seismic considerations. This resulted in inadequate transverse reinforcement, no confinement, no seismic detailing, and lap-splices in the plastic hinge zone of the bent. The representative bridge bent consists of two circular columns per bent, a rectangular cap beam and rectangular pile cap footings. The column longitudinal reinforcement ratio is $\rho_{\mathrm{L}}=1.2 \%$, which is barely above the minimum required by AASHTO $\left(\rho_{\mathrm{L}}>1 \%\right)$. Conversely, the column deficiencies are vast. The provided column shear reinforcement $\left(\rho_{\mathrm{s}}=0.2 \%\right)$ does not meet the code requirement $\left(\rho_{\mathrm{s}}>\right.$ $0.5 \%$ ). The column confinement is almost inexistent since \#4 circular hoops spaced at 12 " were provided. Moreover, lap splices can be found in expected plastic hinge zones and no seismic detailing was specified. The specified material properties were $3300 \mathrm{psi}$ as compressive strength of concrete at 28 days and Grade 40 steel. Typical details for the representative multi-column RC bridge bent following the criteria outlined in the previous section is illustrated in Figure 7.1.

\subsubsection{Assessment of the Representative Bridge}

According the FHWA retrofit design manual (FHWA 2006) in order to identify evaluation methods and retrofitting measures the performance-based seismic retrofit categories (SRC) need to be determined. This retrofit categories depend on the bridge importance, the anticipated life, which is one of the factors in deciding the extent of retrofitting, and the seismic hazard. For the representative bridge and most of the deficient bridges in Oregon a standard importance category is recommended. The anticipated service life (ASL) lies in the range from 16 to 50 years, which represents an ASL 2. Next, the performance level for the bridge needs to be determined. The Oregon Bridge Design and Drafting Manual (BDDM) in its 2014 version recommends a dual performance level. The two-level performance criteria for existing bridges recommended by ODOT are "Life Safety" under a 1000-year return period earthquake (7\% probability of exceedance in 75 years) and "Operational" under a 500-year return period event (14 percent probability of exceedance in 75 years). The inclusion of this additional lower level performance 
criteria ("Operational") is above the standard performance requirements prescribed by AASHTO. Moreover, ODOT raised the lower earthquake ground motion level from a 100-year return period event ( 50 percent of probability of exceedance in 75 years) found in the FHWA retrofit manual to a 500-year event.

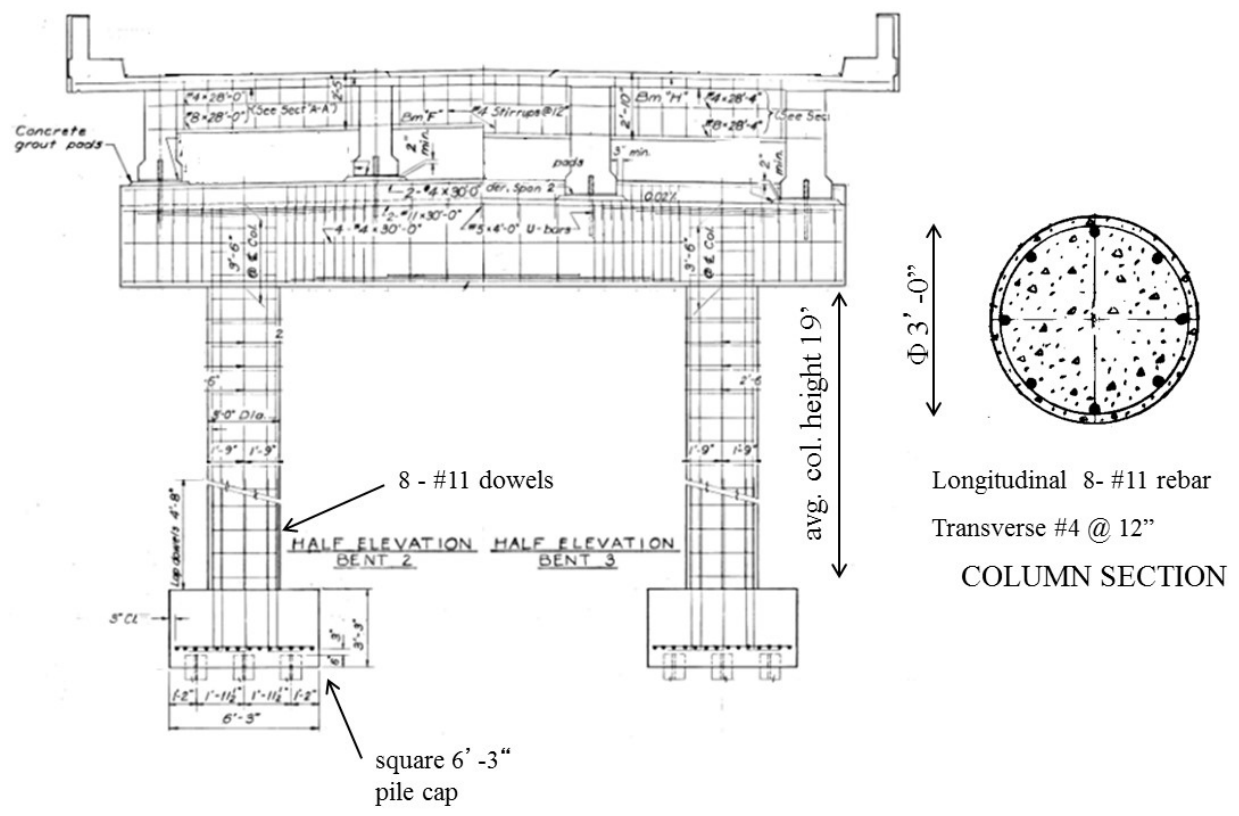

Figure 7.1: Representative RC bridge bent

The seismic hazard level used in this study does not correspond to any specific location, instead the response spectrums used in the study aim to be representative of a vast number of prestressed concrete stringer/girder bridges in Oregon. The design response spectrums were developed using the two-point method and are depicted in Figure 7.2

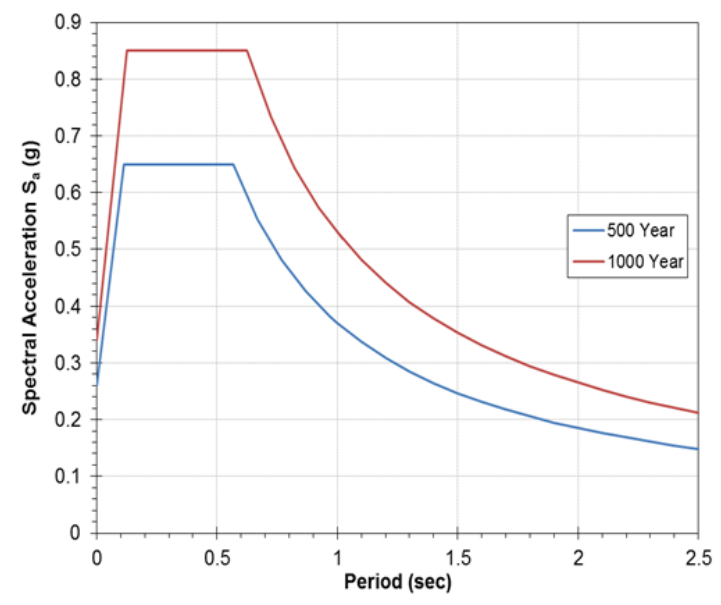

Figure 7.2: Representative response spectrums 
Once the spectral accelerations are obtained the seismic retrofit category (SRC) can be determined. For the representative bridge the SRC is as follows:

- $\quad$ SRC B for the 500-year event

- $\quad$ SRC C for the 1000-year event

The methods of evaluation for the two SRC vary from a minimum analysis of component capacity/demand method to more refined analysis such nonlinear procedures (Pushover method and nonlinear time history analysis). In the assessment of the representative bridge bent two methods are used, namely, the displacement demand/capacity method and the pushover method.

In the displacement demand versus capacity method the guidelines described in AASHTO (AASHTO 2009) were used. The displacement capacity was obtained the approximation given by Eq. (7.1) for the seismic design category (SDC) C since the bridge under the 500-year event can be categorized in this SDC.

$$
\begin{gathered}
\Delta_{C}{ }^{L}=0.12 H_{o}(-2.32 \ln (x)-1.22) \geq 0.12 H_{o} \\
x=\frac{\Lambda B_{o}}{H_{o}}
\end{gathered}
$$

Where, $H_{o}$ is the column clear height, $B_{o}$ is the column diameter and $\Lambda$ is a factor that accounts for the column end restraint condition, 1 for fixed-free and 2 for fixed top and bottom.

However, this equation was developed to represent the displacement capacity of well detailed columns designed in accordance with the AASHTO design specification. In order to overcome this problem, Imbsen (Imbsen 2006) recommended the use of Equations (7.2) and (7.3) for lapsplice and poor confinement columns, respectively. The results of this equations are shown in Table 7.1. From that table can be observed that the capacity in the transverse direction is very limited and need special consideration in the retrofit program.

$$
\begin{aligned}
\Delta_{C}{ }^{L} & =4.0 \cdot(0.0013)^{x} \\
\Delta_{C}{ }^{L} & =3.9 \cdot(0.0070)^{x}
\end{aligned}
$$

Table 7.1: Displacement capacity of the representative bridge bent

\begin{tabular}{c|c|c|c}
\hline Direction & SDC C & Lap-Splice & $\begin{array}{c}\text { Poor } \\
\text { Confinement }\end{array}$ \\
\hline Transverse & 3.09 & 0.96 & 1.64 \\
\hline Longitudinal & 6.65 & 3.03 & 3.87 \\
\hline
\end{tabular}

Displacement demands for the displacement demand versus capacity method were based on equivalent elastic analysis. Further, the displacements were magnified through the use of the 
displacement magnification factor for short period structures, $R_{d}$ as shown in Eq. (6.1). Comparison between displacement demands (Table 7.2) and capacities lead to the conclusion that the bridge is deficient in the transverse direction.

Table 7.2: Displacement demand in representative bridge bent

\begin{tabular}{c|c|c}
\hline Return Period & Transverse Demand (in) & Longitudinal Demand (in) \\
\hline 500 years & 2.2 & 1.1 \\
\hline 1000 years & 2.9 & 1.7 \\
\hline
\end{tabular}

In order to find the capacity and demand from more refined analysis. A nonlinear static procedure referred to as pushover analysis was performed using the expected material properties. Expected material properties can be determined by applying factors to the specified properties. Factors of 1.3 and 1.1 are recommended in the literature for the compressive strength of concrete and the yield stress of steel, respectively. Therefore, a compressive strength of $4290 \mathrm{psi}$ and a yield stress of $44 \mathrm{ksi}$ were used to model the concrete and steel, respectively. The pushover analysis was carried out using SAP2000. The column and beam inelastic behavior was modeled by using the Caltrans idealized moment-curvature analysis. The results of the pushover analysis demonstrated that the performance of the representative bridge is below the performance criteria stablished by ODOT as shown in Table 7.3.

Table 7.3: Performance of representative bridge

\begin{tabular}{c|c|c|c}
\hline Return Period & Performance point (in) & Performance level & Performance required \\
\hline 500 years & 1.7 & Life Safety & Operational \\
\hline 1000 years & 2.3 & Collapse & Life Safety \\
\hline
\end{tabular}

\subsubsection{BRB Design}

In order to retrofit the deficient representative bridge through the inclusion of buckling restrained braces, the following considerations were made:

- The BRB length and angle are limited by the dimensions of the representative bridge bent, which has a span length, $L=240$ in and height, $H=243$ in. A brace angle $(\theta)$ of 47.5 degrees was considered appropriate for this application.

- An inertial mass of $1.813 \mathrm{kip}-\mathrm{s}^{2} /$ in was obtained from the superstructure dead load.

- A response spectrum with maximum spectrum acceleration $\left(\mathrm{S}_{\mathrm{a}}\right)$ of $0.65 \mathrm{~g}$ with $\mathrm{T}_{\mathrm{s}}$ equal to $0.49 \mathrm{sec}$ for the 500 -year event and $0.85 \mathrm{~g}$ with $\mathrm{T}_{\mathrm{s}}$ equal to $0.53 \mathrm{sec}$ for the 1000-year event were considered as depicted in Figure 7.2. Such spectrum accelerations were assumed in an effort to represent acceleration demands of a vast number of bridges in the State of Oregon.

- An expected compressive strength of concrete equal to $4.3 \mathrm{ksi}$ and an expected yield stress of $44 \mathrm{ksi}$ for the steel reinforcement were considered. For the brace, a yield stress of $42 \pm 4 \mathrm{ksi}$ was considered appropriate for this application. 
- The obtained as-built bent parameters from the pushover analysis were: $\delta_{\mathrm{y}}{ }^{\mathrm{B}}=0.98$ inches and $\mathrm{k}_{\mathrm{i}}^{\mathrm{B}}=252 \mathrm{kip} / \mathrm{in}$.

Following the procedure described in Chapter 6 , the minimum required stiffness for the BRB was approximately computed as $1200 \mathrm{kip} / \mathrm{in}$. Thus, one possible BRB design would require a steel core area of $3.8 \mathrm{in}^{2}$ and a reduced section length $\left(\mathrm{L}_{\mathrm{c}}\right)$ of $60 \mathrm{in}$. The total system demands were then calculated as: $\delta^{\mathrm{R}}{ }_{500}=0.83$ inches and $\delta^{\mathrm{R} 1000}=1.08$ inches. Other BRB designs are also acceptable if the strength of the concrete elements is sufficient. In this case, the steel core area was reduced in order to satisfy capacity checks, which resulted in minor inelastic excursions for the 1000-year event as illustrated in Figure 7.3. The target performance levels were achieved, resulting in an "Operational" performance level for the bridge following the design level 500 year as well as 1000 year earthquake.

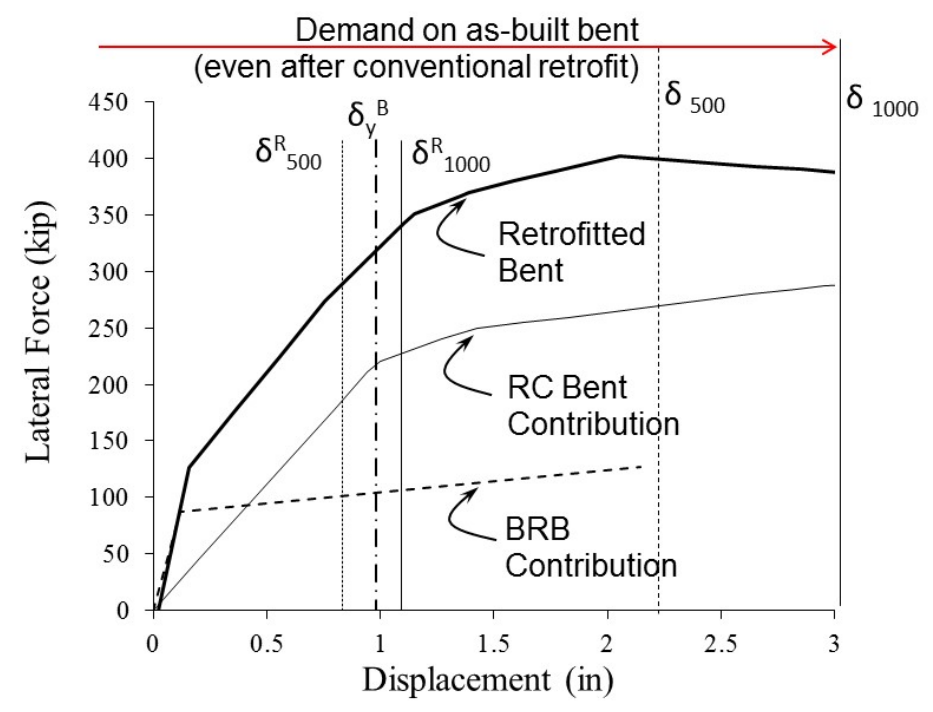

Figure 7.3: Representative bridge behavior

\subsection{EXPERIMENTAL PROGRAM}

\subsubsection{General Description}

In this study, the cyclic performance of a reinforced concrete bridge bent retrofitted using buckling restrained braces (BRBs) was experimentally evaluated using quasi-static cyclic loading that tries to replicate the displacement demands imposed by subduction zone mega earthquakes. The large-scale reinforced concrete bent specimen measured 122.5 inches by 118 inches and consisted of two circular cross section columns and a rectangular cap beam. The columns were subjected to constant axial load. The buckling restrained braces were designed as a replaceable element following the structural fuse concept. The brace was secured to the frame using steel brackets (gusset plates) and post-installed adhesive anchors. 


\subsubsection{Test Program}

The experimental program consisted of three test intended to represent half-scale models of a typical RC bridge bent. Two BRB designs were considered in the study in an effort to assess the influence of BRB stiffness on the overall structural performance. In the third test the bent was tested in the as-built condition. The performance of these specimens was intended to reveal vulnerabilities in existing deficient RC bents and assess the behavior of its retrofitted condition by using Buckling Restrained Braces.

\subsubsection{Test Specimens}

\section{As-Built RC Bridge Bent}

In order to represent a typical RC bridge bent in the State of Oregon, a half-scale reinforced concrete bent of the representative bridge illustrated in Figure 7.1 was designed. Similitude laws were used in order to design the half scale RC bent hereinafter referred to as "As-built Bent". Table 7.4 illustrates the scaling process and includes relevant dimensions and reinforcement details for the test specimen.

The half-scale RC bent consists of two circular columns per bent and a rectangular cap beam. The column longitudinal reinforcement ratio, $\rho_{\mathrm{L}}=1.2 \%$, and the transverse reinforcement ratio, $\rho_{\mathrm{s}}=0.2 \%$, are the same to the reinforcement ratios found in the representative bridge bent, hereinafter referred to as "Prototype". Moreover, lap splices of 40 times the diameter of the longitudinal bar $\left(\mathrm{d}_{\mathrm{b}}\right)$ were used at the base of the columns to be representative of the prototype condition.

The longitudinal reinforcement in each specimen consisted of $10 \# 5$ bars equally spaced. The transverse reinforcement was deformed wire D5 spaced at 6 inches center to center. Lap splices were located at the base of the test specimens through the incorporation of 10 \#5 dowels. The lap splice length was 25 inches, which corresponds to 40 times the diameter of the longitudinal steel reinforcement $\left(40 \mathrm{~d}_{b}\right)$. The longitudinal reinforcing steel used to construct the test specimens consisted of Grade 40 deformed bar conforming to the American Society of Testing and Materials (ASTM) designation A615. On the contrary, the transverse steel consisted of deformed wire conforming the ASTM designation A496.

Normal weight concrete was used to construct the test specimens with a target 28-day strength of 3300 psi. The concrete cover was 1 inch for columns and $3 / 4$ for the cap beam. Standard compression testing of 6-inch by 12-inch concrete cylinders was performed at 7-day, 28 days and at the day of test completion. The average of the concrete cylinder tests are shown in Table 7.5 and Table 4.3. A sketch of the As-built bent reinforcement detailing and cross section is shown in Figure 7.4. 

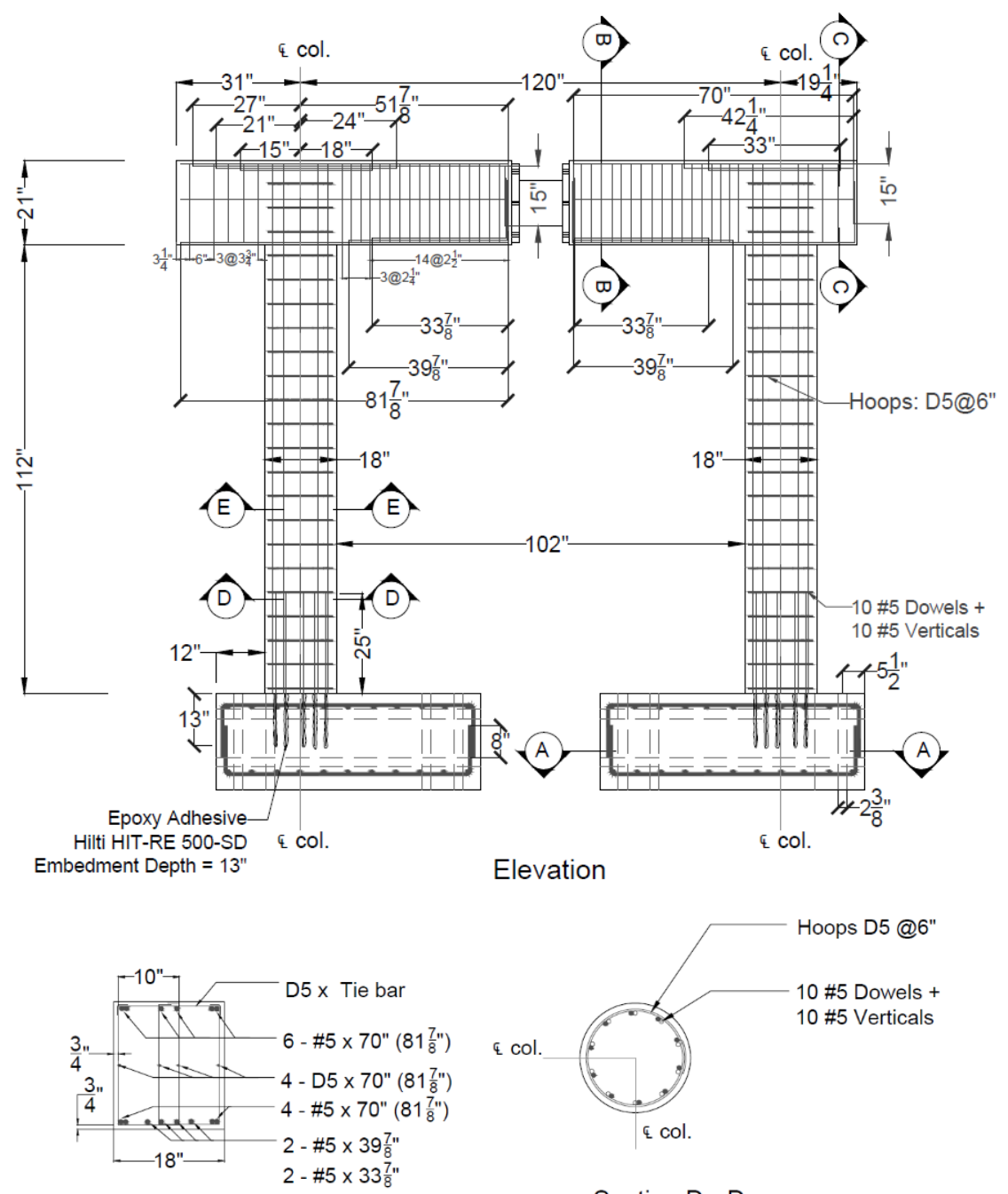

Section B - B

Section D - D
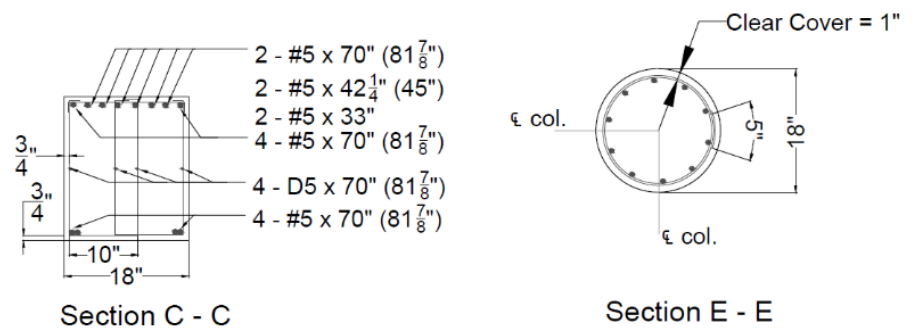

Section E - E
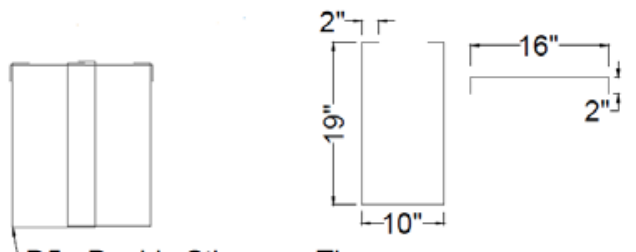

Stirrup and Tie Details

Figure 7.4: Geometry and reinforcement of RC Bridge bent specimens 
Table 7.4: Dimension of representative bridge and test specimen.

\begin{tabular}{|c|c|c|c|}
\hline Parameter & Prototype & Specimen & $\begin{array}{c}\text { Scale } \\
\text { Factor }\end{array}$ \\
\hline Column Diameter (in) & 36 & 18 & 0.5 \\
\hline Column Height (in) & 224 & 112 & 0.5 \\
\hline Beam Depth (in) & 42 & 21 & 0.5 \\
\hline Beam Width (in) & 36 & 18 & 0.5 \\
\hline Beam Length (in) & 240 & 120 & 0.5 \\
\hline$\rho_{\text {Long }}(\%)$ & 1.2 & 1.2 & 1 \\
\hline Hoop Spacing (in) & 12 & 6 & 0.5 \\
\hline$\rho_{\text {Trans }}(\%)$ & 0.2 & 0.2 & 1 \\
\hline $\begin{array}{l}\text { Lap splice }\left(\mathrm{d}_{\mathrm{b}}\right) \\
\mathrm{d}_{\mathrm{b}} \text { : diameter of longitudinal } \\
\text { reinf. }\end{array}$ & 40 & 40 & 1 \\
\hline Concrete cover columns (in) & 2 & 1 & 0.5 \\
\hline Concrete cover cap beam (in) & 1.5 & 0.75 & 0.5 \\
\hline Axial Load $\left(\% \mathrm{Ag}_{\mathrm{g}} \mathrm{f}_{\mathrm{c}}\right)$ & 10 & 10 & 1 \\
\hline Yield stress, $\mathrm{f}_{\mathrm{y}}(\mathrm{ksi})$ & 40 & 40 & 1 \\
\hline Compressive strength , $\mathrm{f}_{\mathrm{c}}(\mathrm{psi})$ & 3300 & 3300 & 1 \\
\hline
\end{tabular}

Table 7.5: Compressive strength of concrete cylinders for deficient RC bent

\begin{tabular}{c|c|c}
\hline \multirow{2}{*}{ Concrete Pour } & \multicolumn{2}{|c}{ Compressive Strength $\left(\mathbf{f}_{\mathbf{c}}{ }^{\prime}\right), \mathbf{p s i}$} \\
\cline { 2 - 3 } & Columns & Beam \\
\hline 7 day & 3961 & 4774 \\
\hline 28 day & 4326 & 4768 \\
\hline $\begin{array}{c}\text { Model 1 } \\
\text { (test day) }\end{array}$ & 4833 & 4776 \\
\hline $\begin{array}{c}\text { Model 2 } \\
\text { (test day) }\end{array}$ & 4971 & 4780 \\
\hline $\begin{array}{c}\text { As-built } \\
\text { (test day) }\end{array}$ & 4975 & 479 \\
\hline
\end{tabular}

\section{Design of Buckling Restrained Braces}

The design of BRBs followed the procedure described in Chapter 6.0, which uses a structural fuse concept as basis for design. The structural fuse concept states that the Asbuilt bent would respond elastically under a damaging earthquake event, i.e. the BRB needs to be designed in such way to reduce the displacement demands on the As-built bent under 500-year and 1000-year earthquake events. This reduction in displacement demand is called retrofit action as illustrated in Figure 6.9. In this figure, the BRB and the As-built RC bent responses are idealized through load-displacement curves. Relevant load-displacement parameters are the yield displacement $\left(\delta_{\mathrm{y}}\right)$ and yield force $\left(\mathrm{V}_{\mathrm{y}}\right)$ denoted by the superscripts BRB, B and R to designate the brace, As-built and retrofitted responses, respectively. The response of the retrofitted bent is no other than adding the contributions of the As-built bent and the BRB since both structural systems act in 
parallel. The design of BRBs is reduced to iterate until the BRB stiffness, the BRB steel core area and the length of the reduced section $\left(\mathrm{L}_{\mathrm{c}}\right)$ are determined.

Two BRB designs were considered in the study in an effort to assess the influence of BRB stiffness on the overall structural performance. The first BRB design, hereinafter referred to Model 1, was designed following the structural fuse concept. The second model, hereinafter referred to Model 2, was designed as a more flexible brace, as compared to Model 1, in order to reflect a common industry practice where the reduced section is equivalent to two thirds of the total BRB length. For the braces, a yield stress of $44.2 \mathrm{ksi}$ given by the BRB manufacturer, a brace angle $(\theta)$ of 48.7 degrees and a BRB length of 130.625 inches were considered appropriate for this application. The obtained parameters for the As-built bent from pushover analysis were $\delta_{\mathrm{y}}{ }^{\mathrm{B}}=0.48$ inches and $\mathrm{V}_{\mathrm{i}}^{\mathrm{B}}=$ 52 kip. A response spectrum with maximum spectrum acceleration $\left(\mathrm{S}_{\mathrm{a}}\right)$ of $0.65 \mathrm{~g}$ with a period at the end of constant design spectral acceleration plateau $\left(\mathrm{T}_{\mathrm{s}}\right)$ equal to $0.49 \mathrm{sec}$ for the 500-year event and $0.85 \mathrm{~g}$ with $\mathrm{T}_{\mathrm{s}}$ equal to $0.53 \mathrm{sec}$ for the 1000 -year event were considered.. The required BRB steel core areas and reduced section lengths $\left(\mathrm{L}_{\mathrm{c}}\right)$ for both models are shown in Table 7.6. Table 7.6 also shows the displacement demands in the retrofitted bent. The response of each resisting system and the retrofitted bent are depicted in Figure 7.5 and Figure 7.6 for Model 1 and Model 2, respectively.

The target performance levels required by ODOT (ODOT 2014) were achieved, resulting in an "Operational" performance level for the Model 1 following the design level 500 year as well as 1000 year earthquake. Further, for Model 2 the Operational performance criteria under the 500-year event and the Life Safety criteria under the 1000 year event were still satisfied.

Table 7.6: Results of BRB designs for Model 1 and 2.

\begin{tabular}{c|c|c|c|c}
\hline \multirow{2}{*}{ Model } & $\begin{array}{c}\text { Steel area } \\
\text { (in } \mathbf{2}^{\mathbf{2}}\end{array}$ & $\begin{array}{c}\text { Reduced Section } \\
\text { (in) }\end{array}$ & \multicolumn{2}{|c}{ Displacement demand (in) } \\
\cline { 3 - 5 } & 1.2 & 30 & $\boldsymbol{\delta}_{\mathbf{5 0 0}}^{\mathbf{R}}$ & $\boldsymbol{\delta}_{\mathbf{1 0 0 0}}^{\mathbf{R}}$ \\
\hline 1 & 1.2 & 88.6 & 0.48 & 0.67 \\
\hline 2 & & 0.76 & 1.06 \\
\hline
\end{tabular}

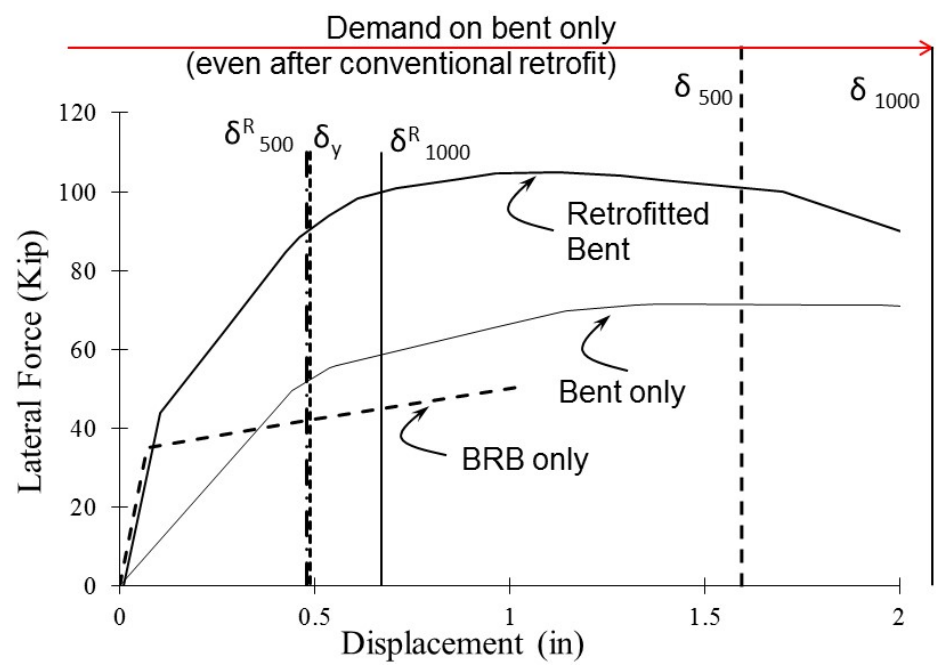

Figure 7.5: Expected load vs displacement behavior for Model 1 


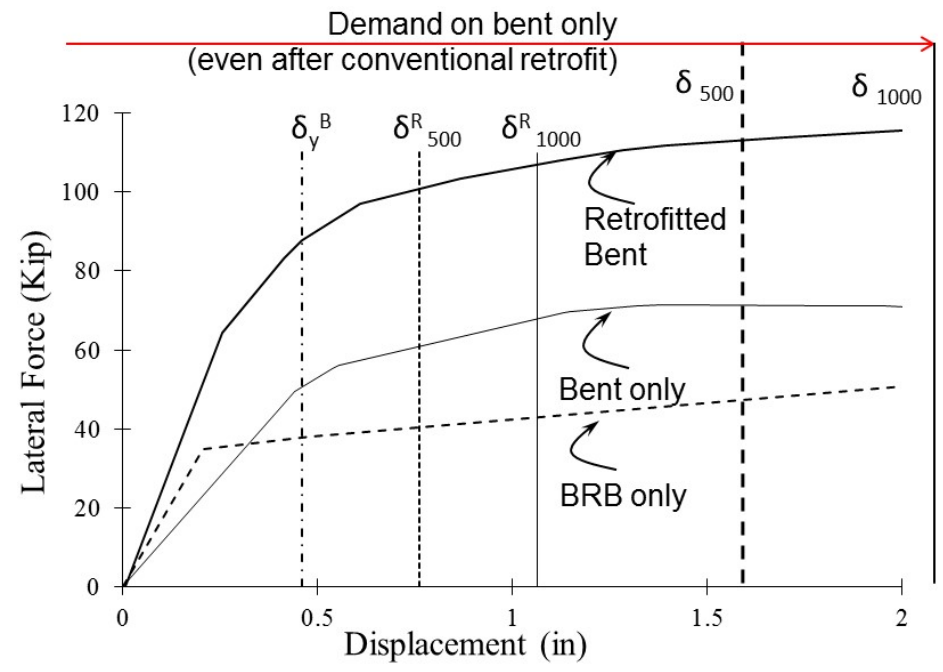

Figure 7.6: Expected load vs displacement behavior for Model 2

\section{Connections}

A novel gusset plate to RC bent connection was utilized in the experimental program. In this connection, the gusset plates were directly connected to horizontal RC elements without interfering with the columns. The authors believe that these were the first tests of this kind on a steel-concrete retrofit connection without the use of an additional steel frame. Figure 7.7 shows the connection used in the experimental program.

Any BRB to gusset plate connection shall be designed to resist the demands based on the adjusted brace strength as per AISC (2010) as shown in Eq. (6.16).

The limit states that were checked for the design of the gusset plate according to AASHTO (2009) and AISC steel manual (2011) are: tensile yielding of Whitmore section, tensile rupture of Whitmore section, block shear in tension, buckling in compression, yield moment strength, plastic moment strength, weld/bolts for gusset to brace connection, and buckling of unsupported edge.

Post-installed adhesive anchors were designed for connecting the gusset plate to concrete elements. The design forces were obtained from the adjusted brace strength and the correspondent gusset plate free body diagram. Tensile and shear strength were considered in the connection design according ACI318 Appendix D (ACI318 2011).

The design procedure for the connections is detailed in the appendices. 


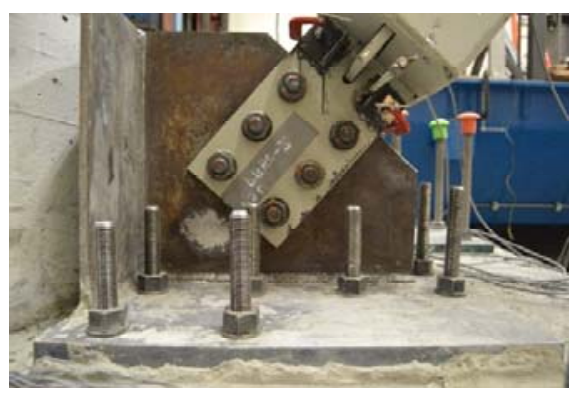

(a)

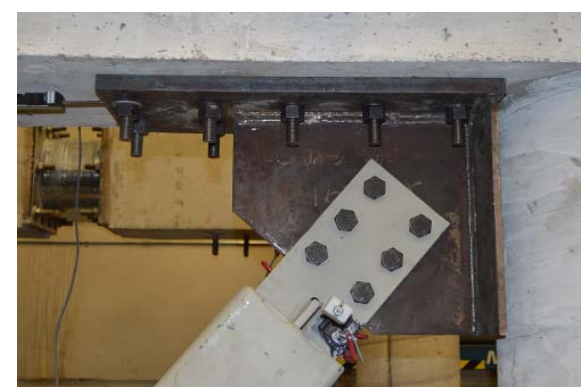

(b)

Figure 7.7: No Gusset Plate to Column Connection (a) Lower connection, (b) Top connection

\subsubsection{Test Setup and Instrumentation}

The experimental setup consists of a half scale model of a typical RC bride bent retrofitted using buckling restrained braces in a diagonal configuration, namely Model 1 and Model 2. The braces in both models have the same material properties and crosssectional area of steel core within the reduced section. However, they differ in length of the reduced section $\left(\mathrm{L}_{\mathrm{c}}\right)$.

The cyclic lateral loading was applied through a horizontal hydraulic actuator capable of applying a maximum load of $220 \mathrm{kip}$ in tension and $300 \mathrm{kip}$ in compression. The actuator was connected to a steel beam on the cap beam. The lateral force was applied under displacement control and load cells were used to monitor the applied load during testing. To simulate the gravity load on bridge columns, $10 \%$ of the column axial capacity $\left(0.10 f^{\prime} \mathrm{cAg}\right)$ was applied through two high-strength rods and hydraulic rams attached to a horizontal steel beam located on top of each column. A six degree of freedom (6DOF) load cell was connected at midspan of the cap beam in order to measure the internal forces that were transmitted from one side of the bent to the other. The footing was secured to the laboratory floor with post-tensioning rods. A schematic representation of the experimental test setup is shown in Figure 7.8, meanwhile the actual test setup is illustrated in Figure 7.9

Failure was defined as a $20 \%$ drop in peak lateral load for each specimen except for Model 1, which was tested up to the expected displacement demand under the 1000-year event $\left(\delta^{\mathrm{R}}{ }_{1000}\right)$. The yield displacement $\left(\delta_{\mathrm{y}}\right)$ was theoretically determined from material properties for Models 1 and 2 and from the pushover analysis for the As-built RC bent. Values of actual yield displacement were then corrected during each test. 


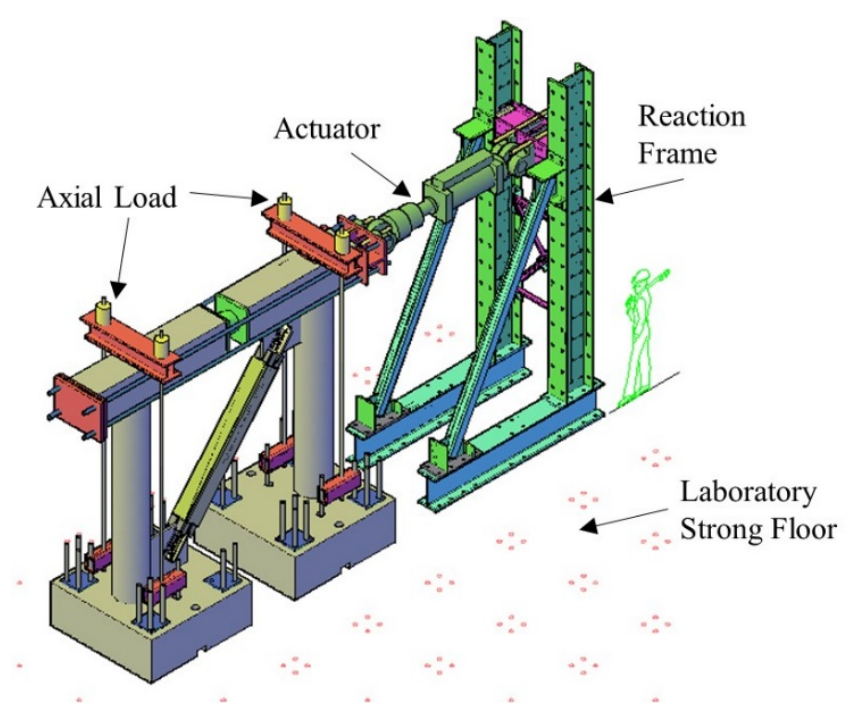

Figure 7.8: Schematic of test setup configuration

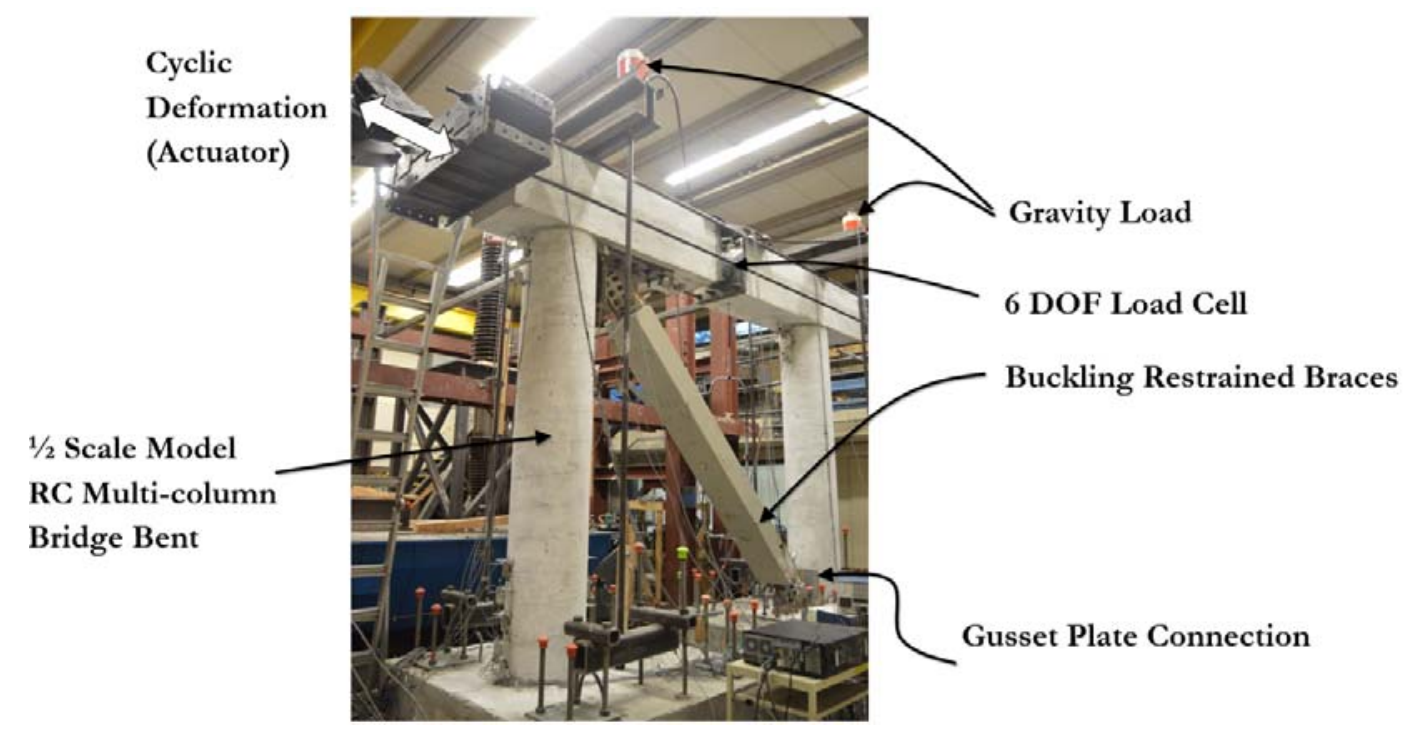

Figure 7.9: Actual test setup

In order to analyze the response of the specimens subjected to cyclic lateral loading, the columns were fully instrumented via strain gages and linear variable displacement transducers (LVDTs) as shown in Figure 7.10 and Figure 7.12, respectively. Strain gages were used to measure the strain at specific points in the specimens. A total of 90 strain gages were mounted. 8 strain gages in the dowels embedded in the footings, 24 in the column dowels, 48 in the longitudinal reinforcement of the columns, 4 in the transverse reinforcement of the columns, 4 in the cap beam, and 2 in the gusset plates. All strain gages were placed at expected plastic hinge zones of columns and cap beam. Installation of all strain gages was performed prior to the placement of the concrete as shown in Figure 7.11. LVDTs were used to measure relative displacements and rotations. For example, rotation of columns, elongation of $\mathrm{BRB}$, relative displacement between 
columns and cap beam, and between columns and footings, etc. A schematic with some of the LVDTs used in this experimental program is depicted in Figure 7.12.

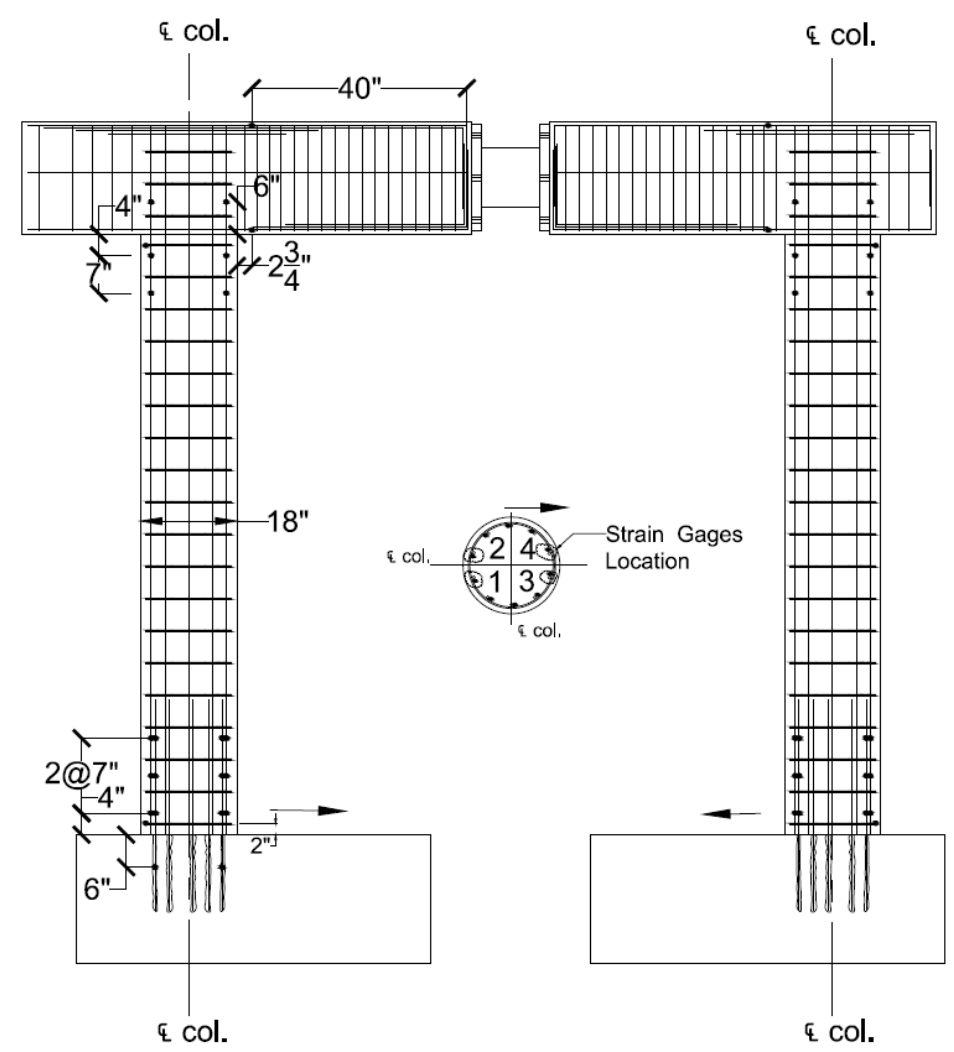

Figure 7.10: Location of strain gages

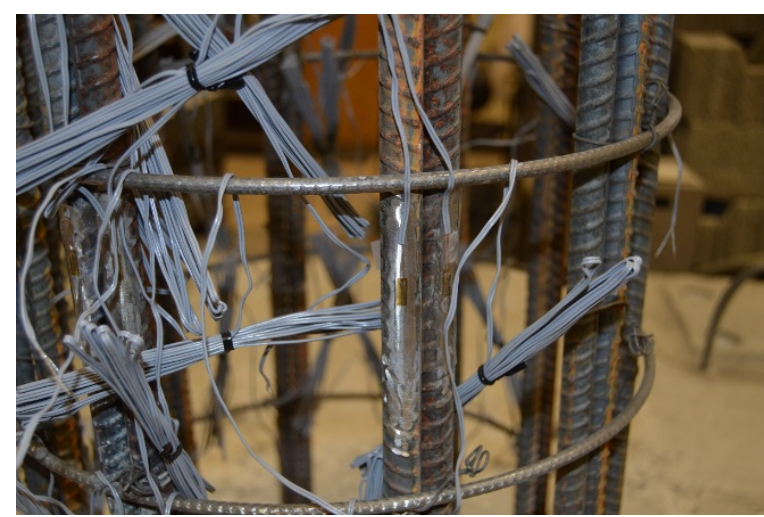

(a)

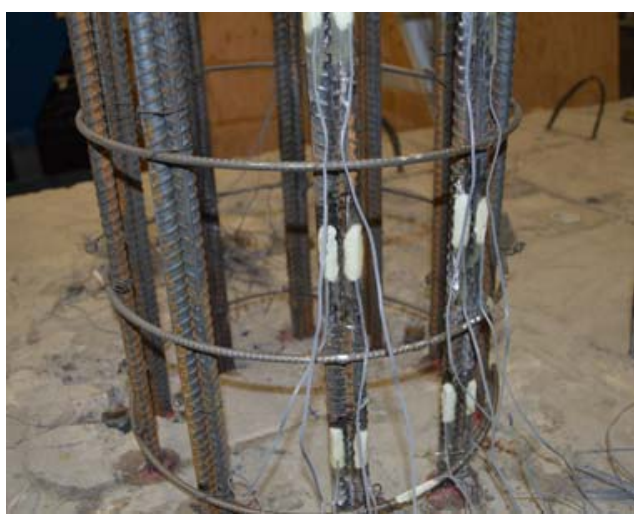

(b)

Figure 7.11: Installation of strain gages. (a) Strain gages on longitudinal reinforcement and dowel, (b) Strain gages with protective coating. 


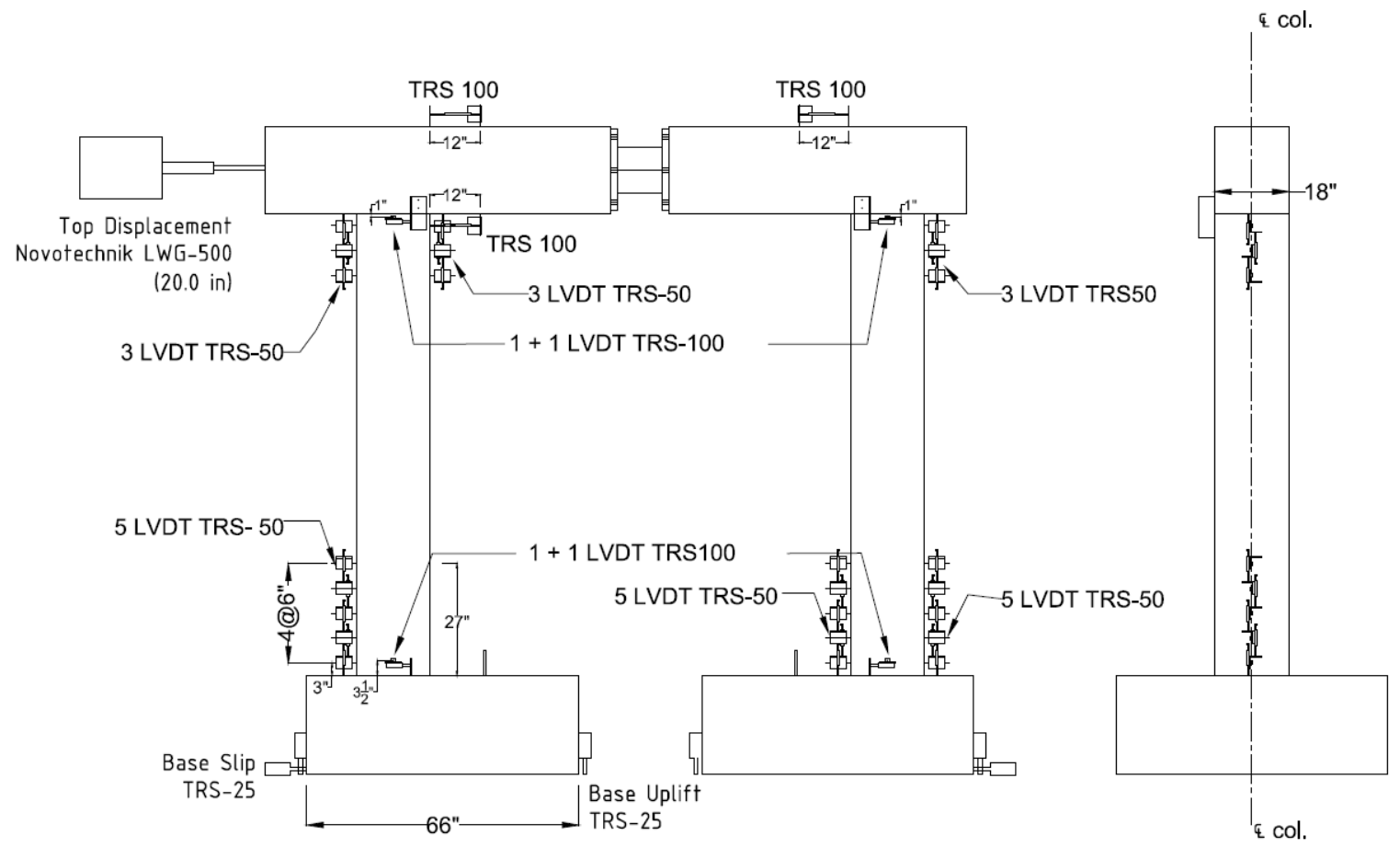

(a)

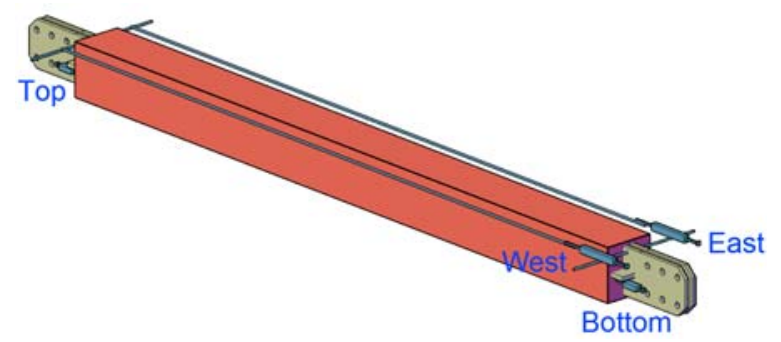

(b)

Figure 7.12: Location of LVDTs. (a) RC Bent, (b) BRB.

\subsubsection{Cyclic Loading Protocols}

Loading of the test specimens was slowly applied using quasi-static cyclic loading protocols aiming to reflect subduction zone earthquake demands up to displacement ductility 8 . For the retrofitted system a new loading protocol was specifically developed for structures of fundamental period of $0.2 \mathrm{sec}$. by using the demand parameters shown in Figure 3.4 and Figure 3.5. This period was found to be more representative of the retrofitted condition because the inclusion of the BRB increases the total system stiffness, which in turns decreases the fundamental period of the structure. For the As-built RC bent the same loading protocol used in the experimental program described in Chapter 4 was utilized. Both protocols are shown in Figure 7.13. 
The horizontal loads were applied under displacement control based on a pattern of progressively increasing displacements, referenced to the horizontal displacement to cause first yield $\left(\delta_{\mathrm{y}}\right)$ in the brace for the Bent/BRB structural system (Model $1 \& 2$ ) and in the columns for the as-built RC bent. The protocols subjected the Bent/BRB system and the as-built bent to cumulative inelastic deformations equal to 351 and 257 times the yield deformation $\delta_{\mathrm{y}}$, respectively. These values of cumulative inelastic deformations are greater than that required in cyclic test for qualification of $\mathrm{BRBs}$, which requires a cumulative inelastic deformation of at least $200 \Delta_{\mathrm{y}}$ (AISC 2010).

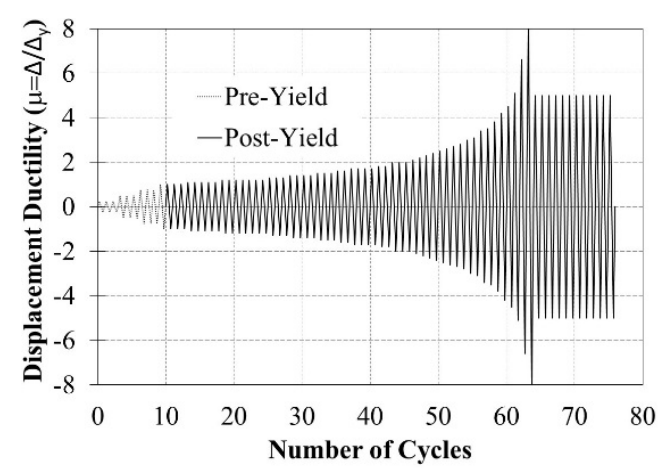

(a)

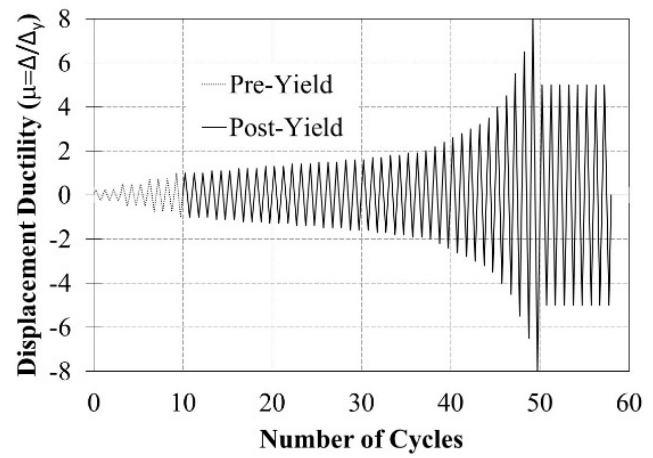

(b)

Figure 7.13: Cyclic loading protocol. (a) Retrofitted bent, (b) As-built RC bent.

\subsubsection{Test Results}

\subsubsection{Model 1}

The buckling restrained brace used in Model 1 was designed using the structural fuse concept described in Chapter 6, which resulted in a considerable stiff BRB as shown in Figure 7.14.

The lateral load vs. displacement hysteresis curve (shown in Figure 7.15) for this specimen indicates high ductile behavior and energy dissipation. Model 1 exhibited a ductile behavior up to a displacement ductility value of 8 , which was the maximum ductility considered in this case. The theoretical yield displacement of the brace was computed as 0.07 inches. However, this yield displacement was corrected during the test in an effort to accommodate the actual response of the test specimen. The experimental yield displacement was 0.083 inches, which is a $19 \%$ increase with respect to the theoretical value. The first horizontal crack of width less than $0.05 \mathrm{~mm}$ occurred at a displacement of 0.25 inches and a lateral load of 79 kip approximately. The peak lateral load was $113 \mathrm{kip}$ and occurred at a lateral displacement of approximately 0.67 inches. The specimen did not exhibited a significant decrease in lateral strength. At the end of the test, only horizontal hairline cracks of width $0.05 \mathrm{~mm}$ were observed at the base and top of columns as shown in Figure 7.16. Once the BRB was removed from the RC bent, it was observed that the gusset plates did not present any trace of damage. The brace was opened in order to observe the damage presented in the steel core. Figure 7.17 shows the 
damage undergoes by the steel core, in which is observed little damage in the intersection between the transition section $\left(\mathrm{L}_{t r}\right)$ and the reduced section $\left(\mathrm{L}_{\mathrm{c}}\right)$. This damage is attributed to high stress concentrations from changing the cross sectional shape of the steel core.

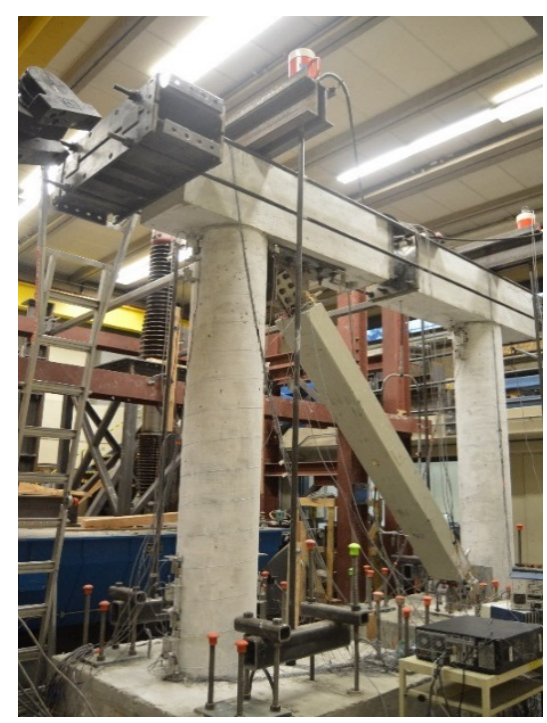

Figure 7.14: Model 1

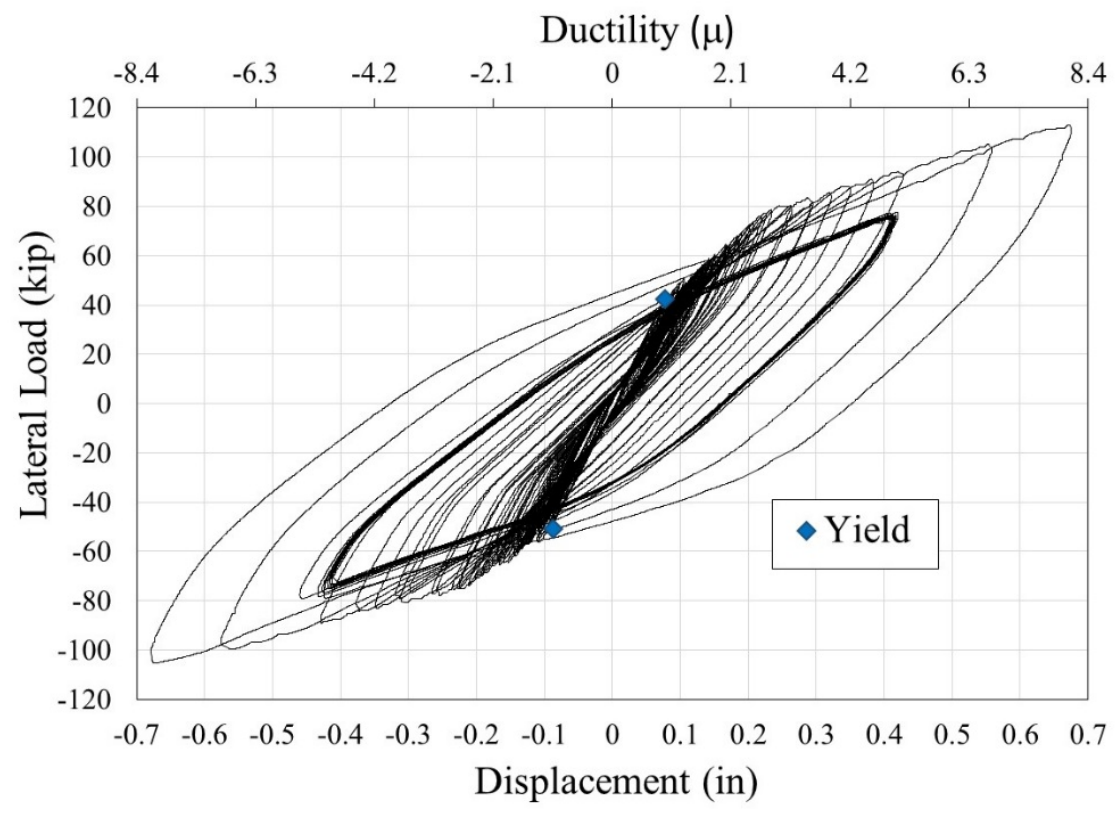

Figure 7.15: Load vs. Displacement curve of Model 1 


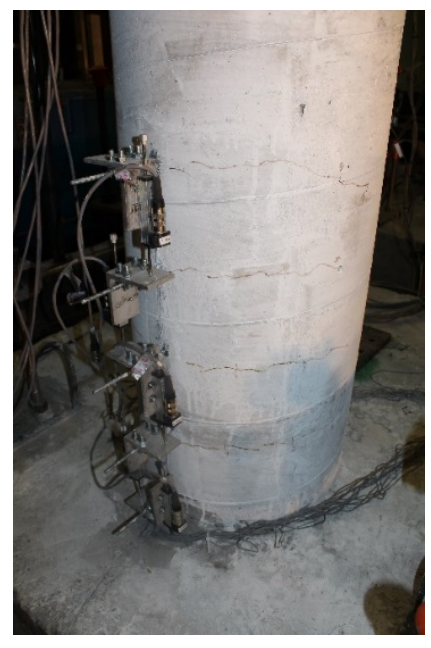

(a)

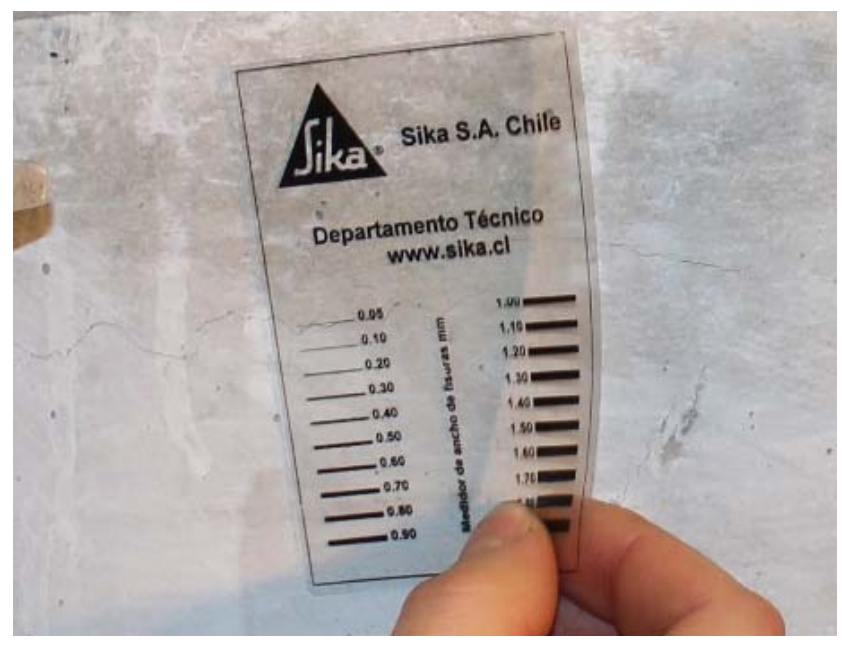

(b)

Figure 7.16: Damage in RC Bent Model 1. (a) Horizontal crack pattern, (b) Crack width
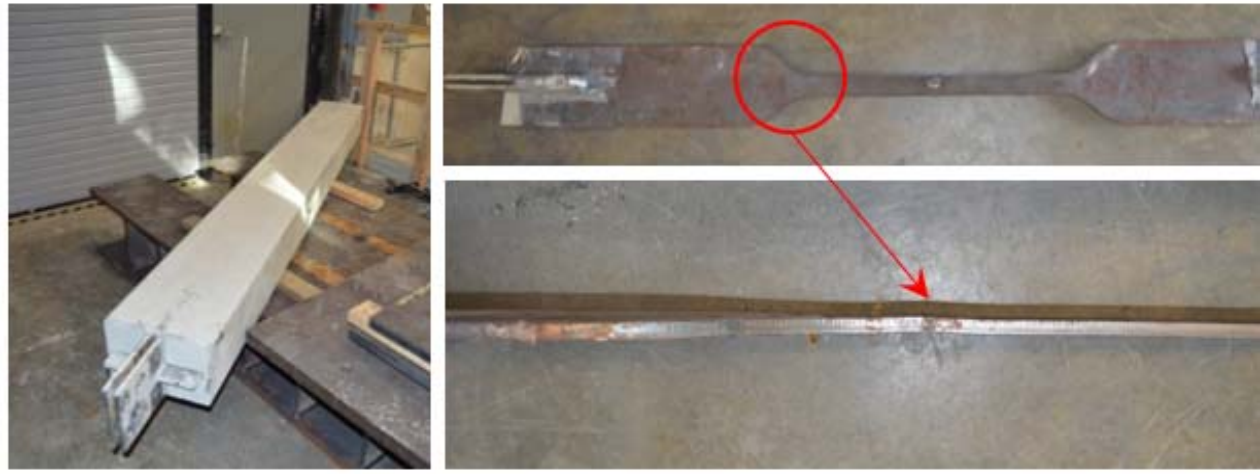

Figure 7.17: Damage in BRB Model 1

\subsubsection{Model 2}

In an effort to assess the influence of BRB stiffness on the overall structural performance, the buckling restrained brace used in Model 2 was designed following a typical design for BRBs, which considers a reduced section equal to two thirds of the brace length. As result, this BRB is more flexible than that used in Model 1. Model 2 is shown in Figure 7.18 . 


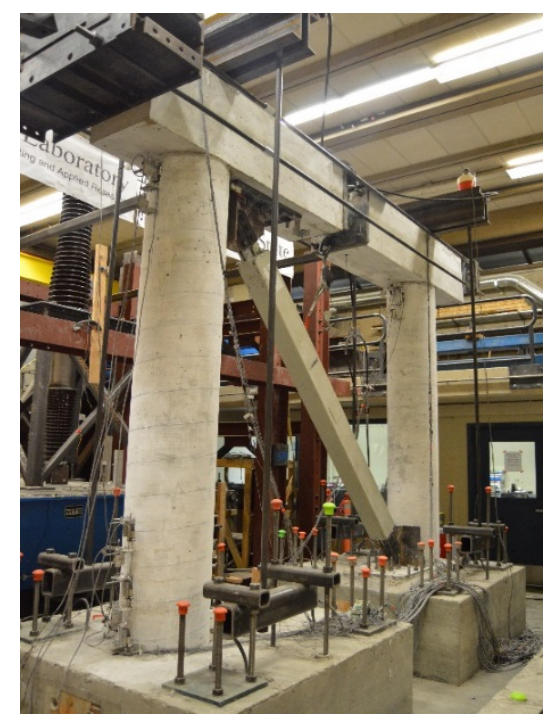

Figure 7.18: Model 2

Figure 7.19 shows the lateral load vs. displacement hysteresis curve for this specimen. The curve indicates high ductile behavior and energy dissipation up to a ductility value of 4.5 , which is equivalent to a displacement of 1.3 inches. The theoretical yield displacement of the brace was computed as 0.2 inches and the experimental one was 0.29 inches, which is a $45 \%$ increase with respect to the theoretical value. The horizontal cracks during the test formed throughout the expected plastic hinge zones of the columns and progressed in length and width as shown in Figure 7.20(a). The maximum horizontal crack width was $0.4 \mathrm{~mm}$. Vertical cracks (width less than $0.6 \mathrm{~mm}$ ) were registered in the cap beam in negative moment areas. As the progressively increasing displacements were applied, the lateral load increased up to -104 kip for the brace compression direction. From that displacement forward, the specimen exhibited a significant decrease in lateral strength during the compression half-cycles. Presumably, buckling of the steel core started developing at this point giving place to fracture of the steel core once the BRB was tensioned after a cycle in compression as shown in Figure 7.19. Once the BRB failed, all the load capacity was carried by the As-built RC bent capacity without considering the BRB contribution.

The peak lateral load was $115 \mathrm{kip}$ and occurred at a lateral displacement of approximately 1.13 inches. At the end of the test, minimal spalling of concrete at the base of the columns was observed as illustrated in Figure 7.20(b). Once the BRB was removed from the RC bent, the gusset plates were inspected and did not exhibit any trace of damage. The brace was opened in order to observe the damage presented in the steel core. Figure 7.21 shows the damage undergoes by the steel core, in which is observed the fracture of the steel core. Even though, this specimen was designed to sustain a maximum displacement of 2.7 inches, the fracture of the steel core occurred at a lateral displacement of 1.2 inches (displacement ductility of 4.5). This mode of failure was attributed to poor confinement in the transition section $\left(\mathrm{L}_{\mathrm{tr}}\right)$ within the brace. 


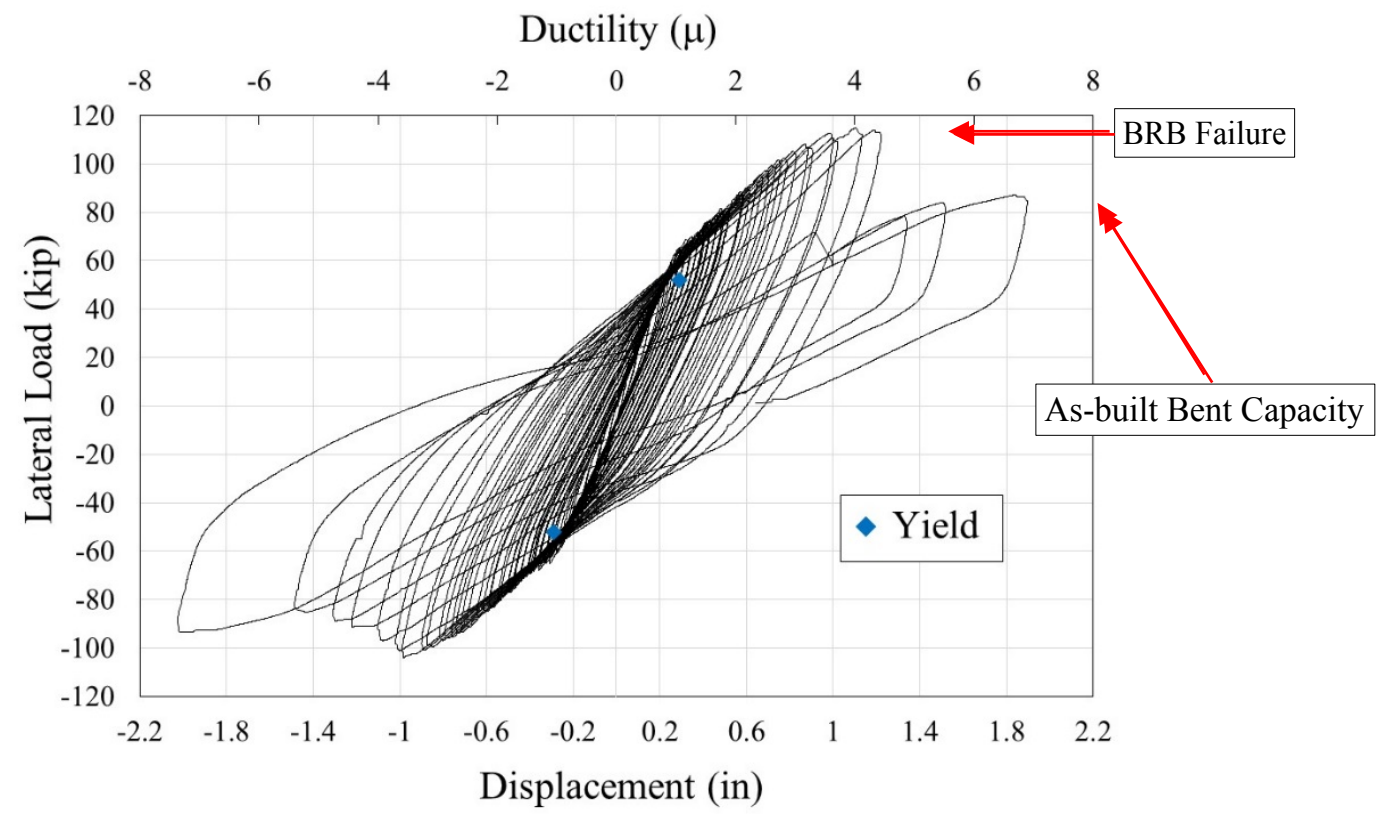

Figure 7.19: Load vs. Displacement curve of Model 2

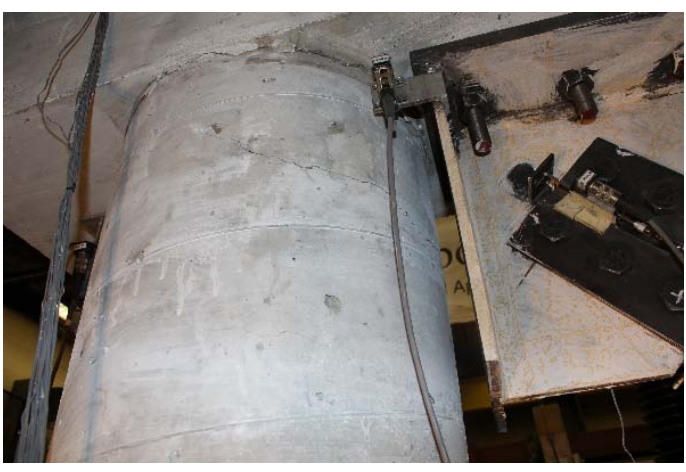

(a)

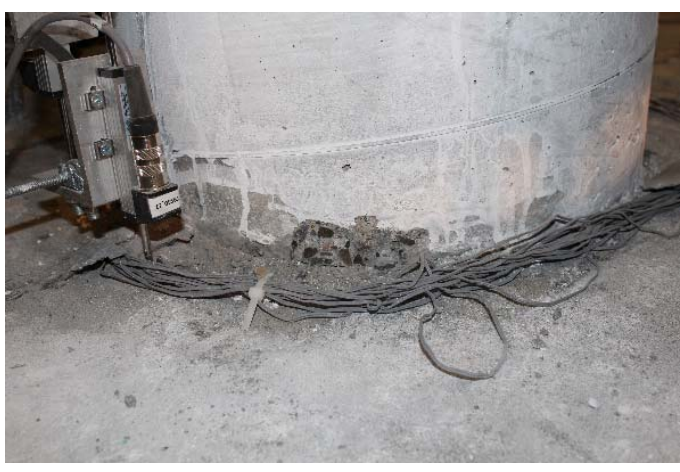

(b)

Figure 7.20: Damage progression in Model 2. (a) Horizontal cracks, (b) Spalling of concrete 

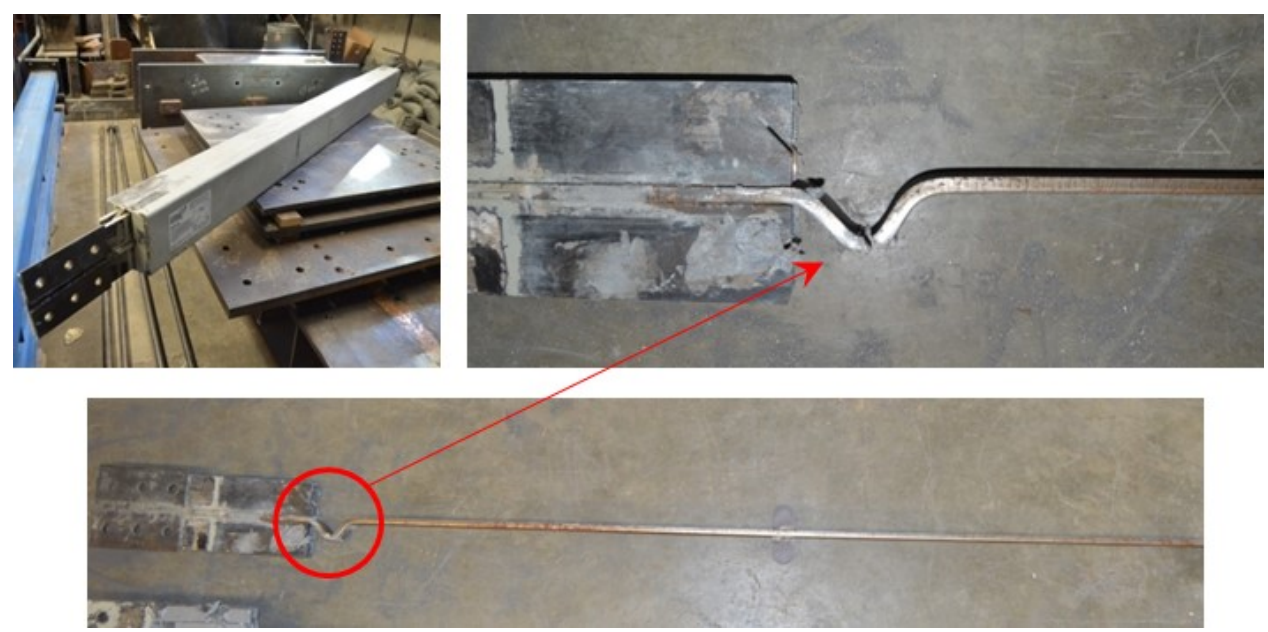

Figure 7.21: Damage in BRB Model 2

\subsubsection{As-Built RC Bent}

Despite all the deficiencies of the As-built RC bent, this bent exhibited a moderately ductile behavior. The initial damage consisted of horizontal cracks that were propagated throughout the height of expected plastic hinge zones to give place to spalling of concrete in early stages. Vertical cracks in the beam increased in width up to $0.8 \mathrm{~mm}$. The ultimate mode of failure was crushing of concrete and buckling of steel reinforcement at the end of testing. Crushing of the concrete at the base and top of the columns began due to flexural loading, exposing the column reinforcement. Once the concrete cover was lost, the longitudinal bars in those regions began to buckle and finally the dowels fracture as shown in Figure 7.23(a) and Figure 7.23 (b), respectively. The buckling of the dowels was observed once the cover of the concrete at the base of the column was completely crushed.

The theoretical yield displacement of the bent was computed using a pushover analysis, which resulted in a yield displacement equal to 0.48 inches. However, this yield displacement was then corrected during the test. The experimental yield displacement was 0.54 inches, which is a $12 \%$ increase with respect to the theoretical value. The experimental yield displacement was determined by using strain gage measurements at the base and top of the columns. Representative strain gage measurements are presented in the appendices.

The lateral load vs. displacement hysteresis curve (shown in Figure 7.22) for this specimen indicates reasonable ductile behavior and energy dissipation. The As-built RC bent was able to attain a maximum displacement of 4.9 inches before the applied load dropped below $80 \%$ of the peak load. The peak lateral load was 70 kips and occurred at a lateral displacement of approximately 2.4 inches. It is worth mentioning that the initial stiffness of the As-built bent is lower since stiffness degradation occurred when the bent was previously tested in a retrofitted state. 


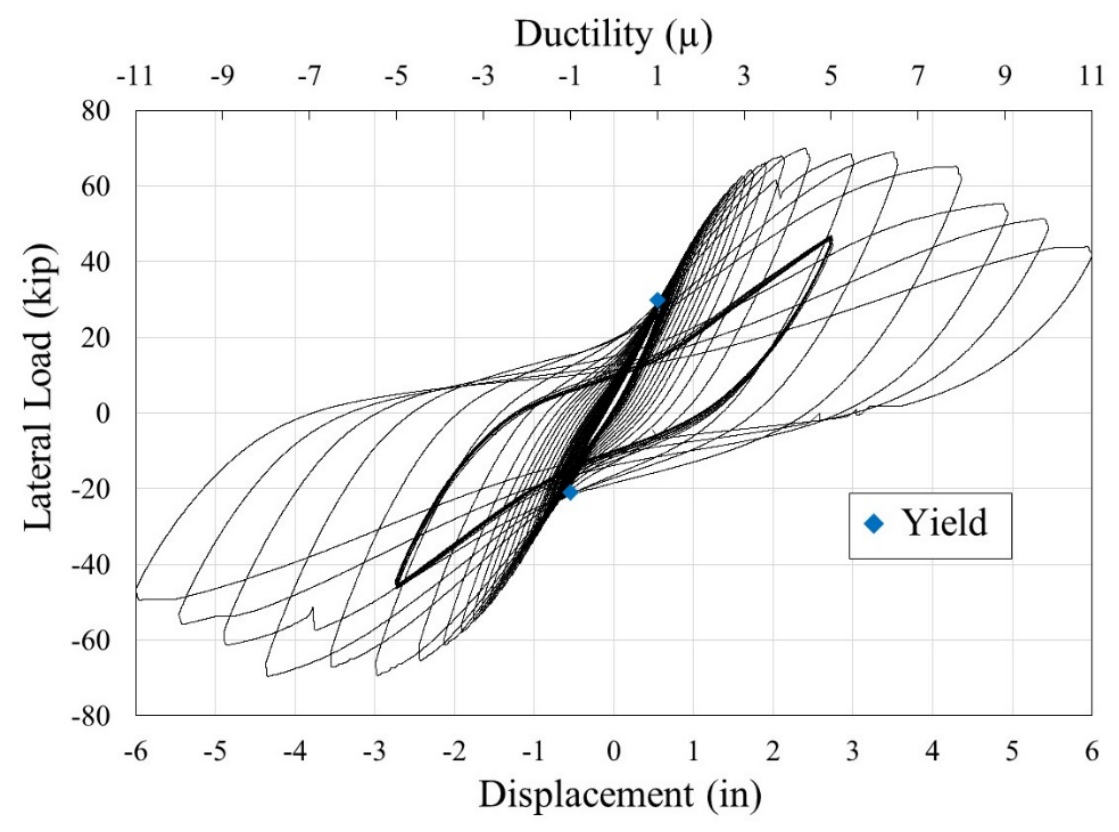

Figure 7.22: Load vs. Displacement curve of As-built RC bent

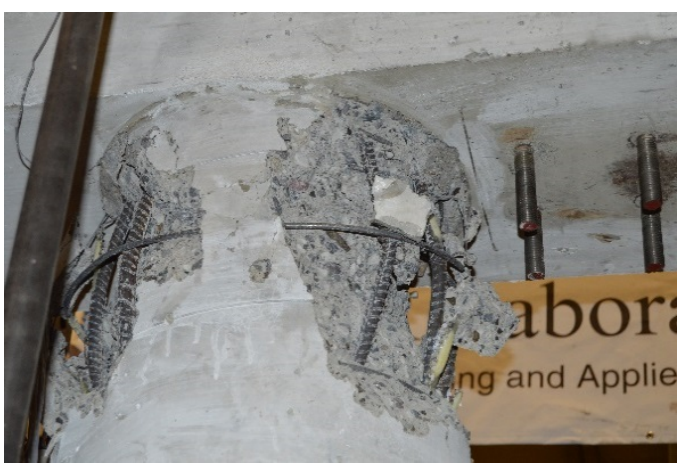

(a)

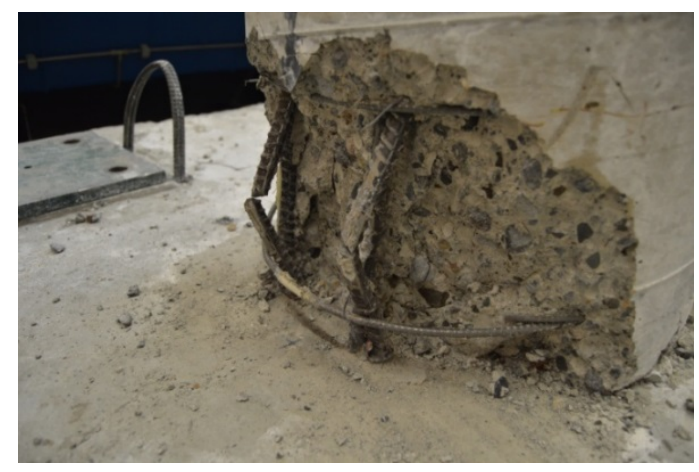

(b)

Figure 7.23: Damage on As-built RC bent. (a) Buckling of Steel, (b) Rupture of steel

\subsubsection{Analysis of Results}

\subsubsection{BRB elongation}

The elongation of the BRB was measured via four LVDTs. Two of them measure the relative displacement between the steel core and the steel casing at the top and bottom connections, referred to as Top and Bottom elongations. The other two, referred to as "West" and "East", were located on the sides of the brace to measure the total elongation. The brace elongation results for Model 1 and Model 2 are shown in Figure 7.24 and Figure 7.25, respectively.

Comparing the top elongation to the bottom elongation it can be concluded that for both models the inelastic behavior was concentrated in the top section. This behavior is even 
more critical for Model2, in which the top elongation was up to 20 times greater than the bottom elongation. As a result, the brace failed at the top part at an elongation of 0.78 inches. The elongation at rupture was lower than our expectations since according the $\mathrm{BRB}$ design the brace should have attained a maximum elongation of 1.78 inches. The unsatisfactory behavior of the BRB in Model 2 was attributed to the poor confinement of the brace within the transition section $\left(\mathrm{L}_{\mathrm{tr}}\right)$.

The measurement collected from the West LVDT agree very well with the one located on the East side as shown in Figure 7.24(b) and Figure 7.25(b). Moreover, The total elongation that was directly collected from the West and East LVDTs agree well with the total elongation calculated adding the Top and Bottom measurements as shown in Figure 7.24(c) and Figure 7.25(c).

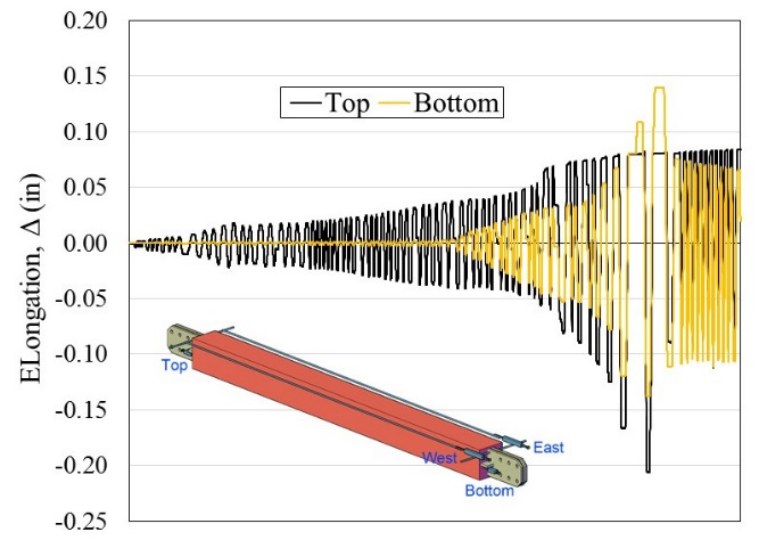

(a)

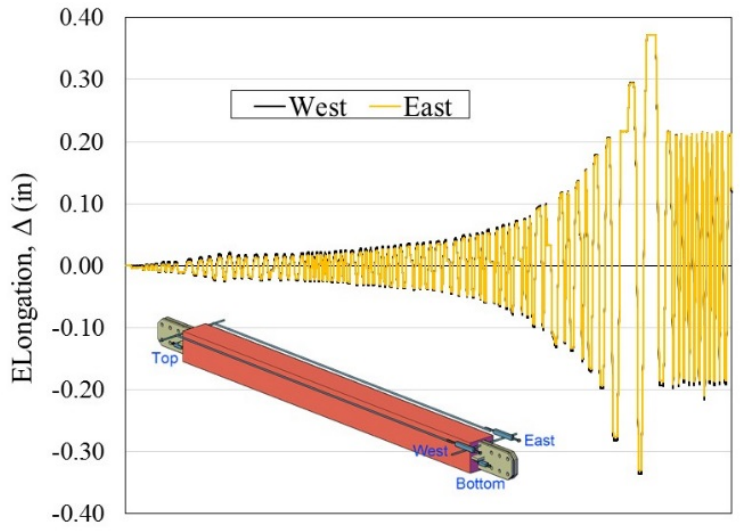

(b)

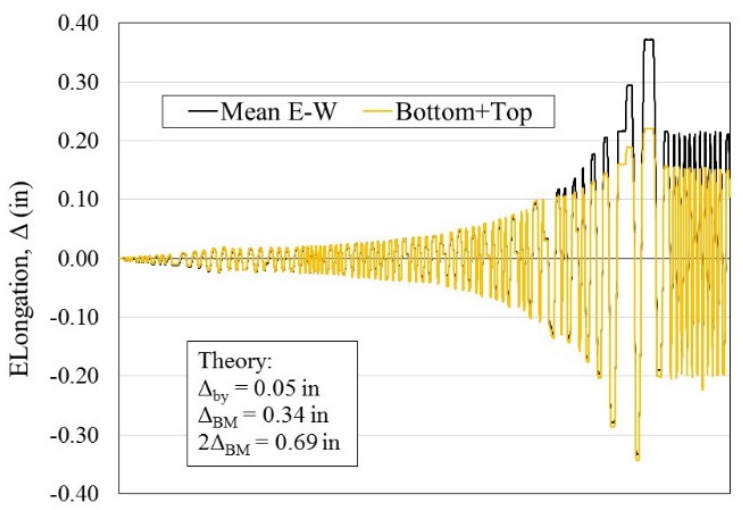

(c)

Figure 7.24: BRB elongation in Model 1. (a) Top and Bottom, (b) West and East, (c) Mean E-W and Bottom+Top 


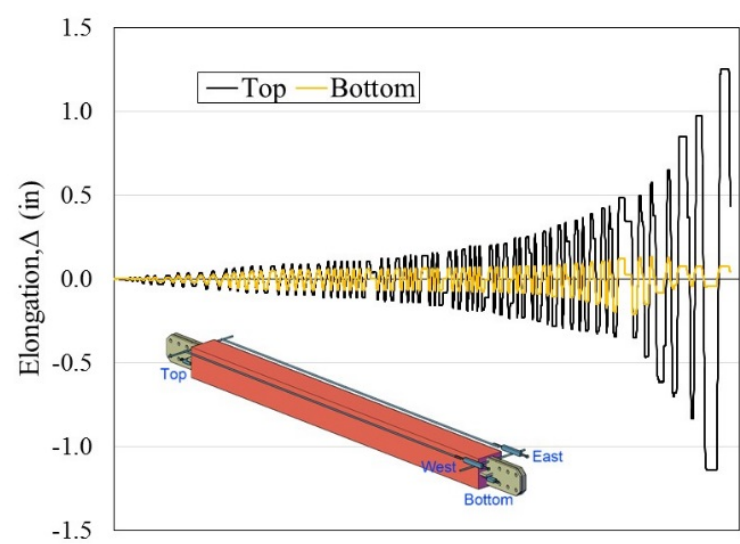

(a)

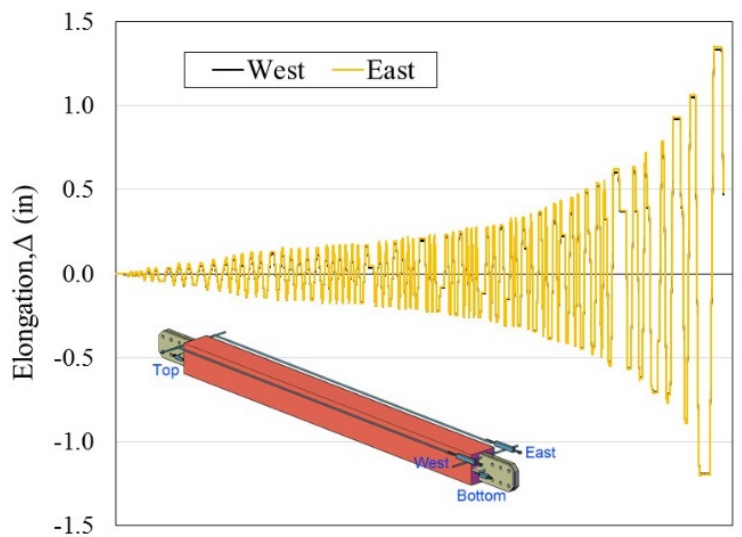

(b)

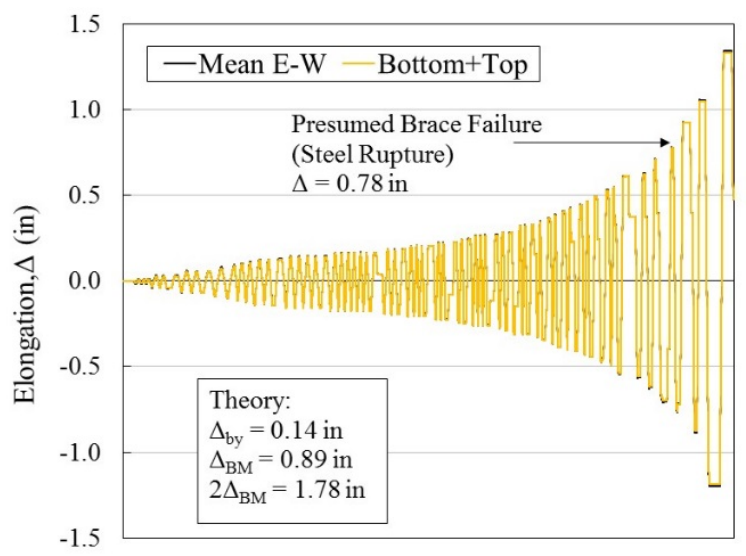

(c)

Figure 7.25: BRB elongation in Model 2. (a) Top and Bottom, (b) West and East, (c) Mean E-W and Bottom+Top

\subsubsection{Comparison to demand}

Figure shows the comparison between the experimental results and the theoretical displacement demands under the 500-year earthquake event and 1000-year event. The target performance levels required by ODOT (ODOT 2014) were achieved, resulting in a fully "Operational" performance level for the Model 1 following the design level 500 year as well as 1000 year earthquake. Further, for Model 2 the Operational performance criteria under the 500-year event and the Life Safety criteria under the 1000 year event were still satisfied. 


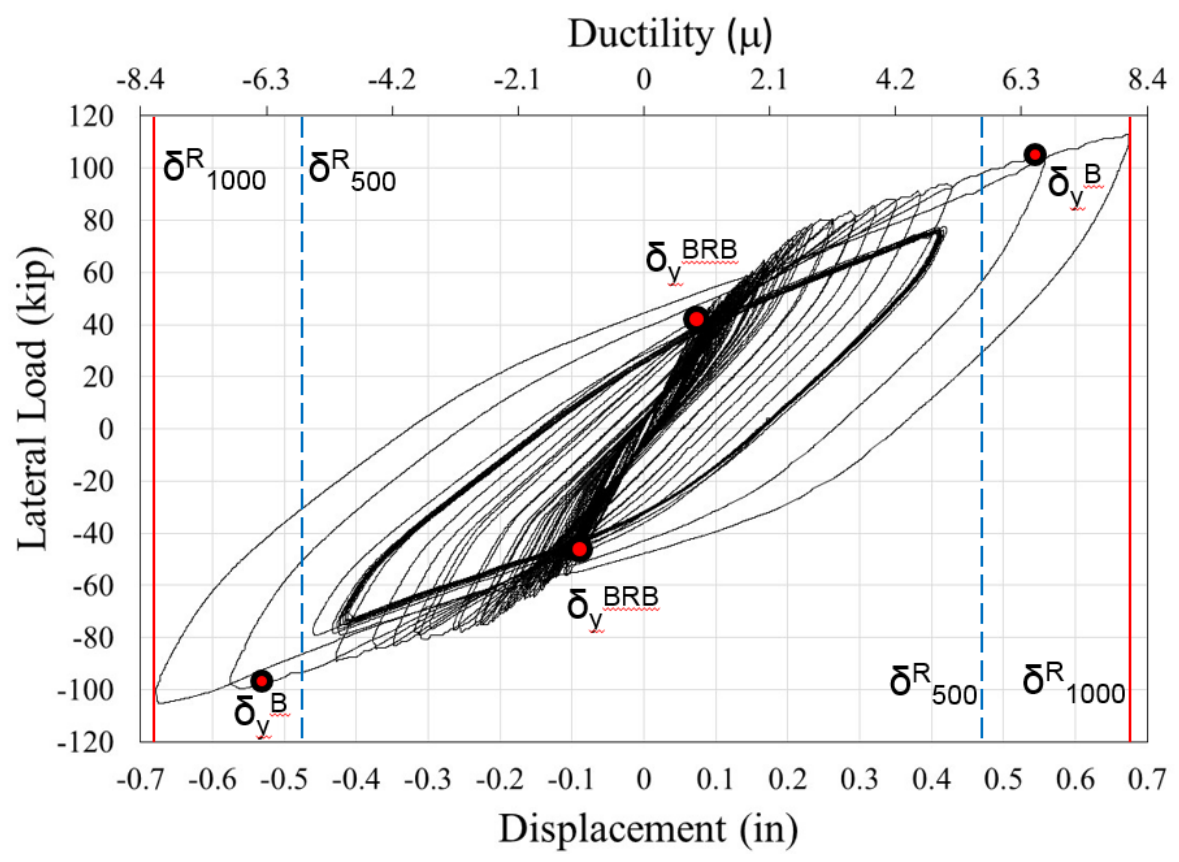

(a)

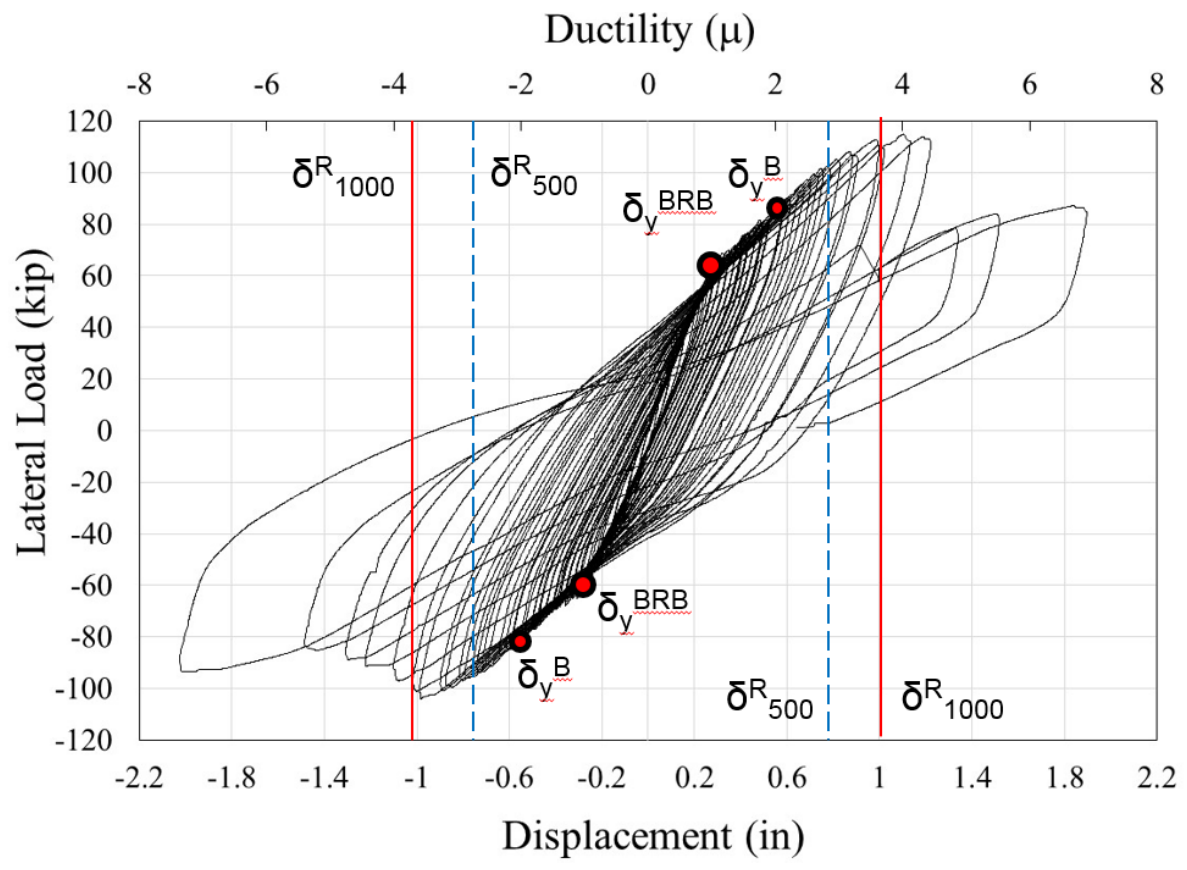

(b)

Figure 7.26: Comparison between experimental results and theoretical demand; (a) Model 1, (b) Model 2 


\subsubsection{Comparison to analytical results}

The load vs displacement envelopes, also referred to as backbone curves, were compared to nonlinear static analyses (pushover) that were carried out during the design of the BRBs. The pushover analyses were performed using SAP2000 (Computers and Structures Inc. 2011). The model used for the nonlinear static analysis is depicted in in Figure 7.27. The following considerations to develop the analytical model were made:

- The inelastic behavior of the columns was modeled assuming the Mander concrete parametric stress-strain curve for the unconfined and confined concrete (Mander 1984) and the Park parametric stress-strain curve for reinforcing steel. The hinges in columns and beam were defined using an idealized moment-curvature behavior as described in Caltrans (Caltrans 2013).

- The BRB was modeled using an axial hinge that was controlled by deformation. The behavior of the axial hinge was modeled as elastic-plastic with hardening as depicted in Figure 6.10.

- All potential plastic hinge locations were considered and were lumped at specific locations shown in Figure 7.27.

- Rigid links were utilized to represent the beam-column joint and gusset plates.

- The comparison between experimental and analytical envelope results are shown in Figure 7.28, Figure 7.29 and Figure 7.30 for Model 1, Model 2 and As-built bent, respectively. The Model 1, that was tested up to the expected displacement demand under the 1000-year event $\left(\delta^{\mathrm{R}}{ }_{1000}=0.67\right.$ inches $)$, showed an excellent fitting between the experimental and analytical load-displacement envelope curves, especially for the initial stiffness and yield displacement. Figure 7.29 compares the experimental result for the Model 2 with those from the pushover analysis using SAP2000. From this figure, it is clear the analytical results agree well with the experimental results until the unexpected failure of the BRB, which was attributed to poor confinement in the transition section $\left(\mathrm{L}_{\mathrm{tr}}\right)$ within the brace. Thus, using a relatively simple nonlinear model the experimental results for the retrofitted condition can be described satisfactorily.

- On the contrary, the As-built bent, showed more displacement capacity as compared to the analytical results but similar strength capacity. It is worth noting that the initial stiffness of the As-built bent is lower since stiffness degradation occurred when the bent was previously tested in a retrofitted state. 


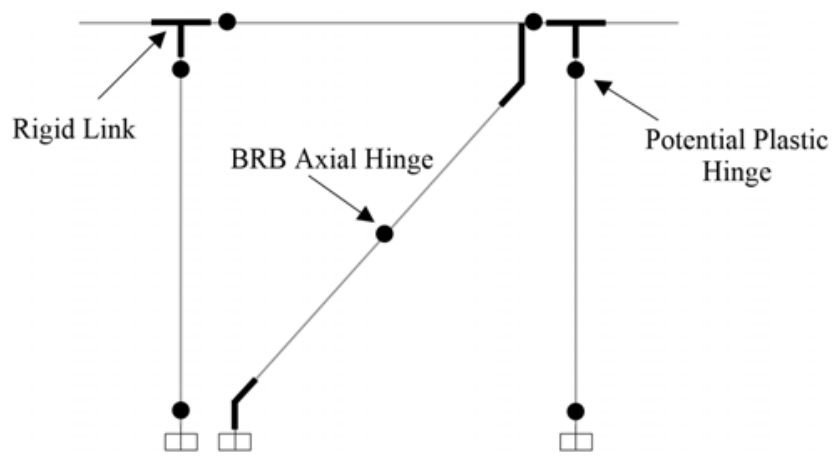

Figure 7.27: Model schematic for the nonlinear static analysis

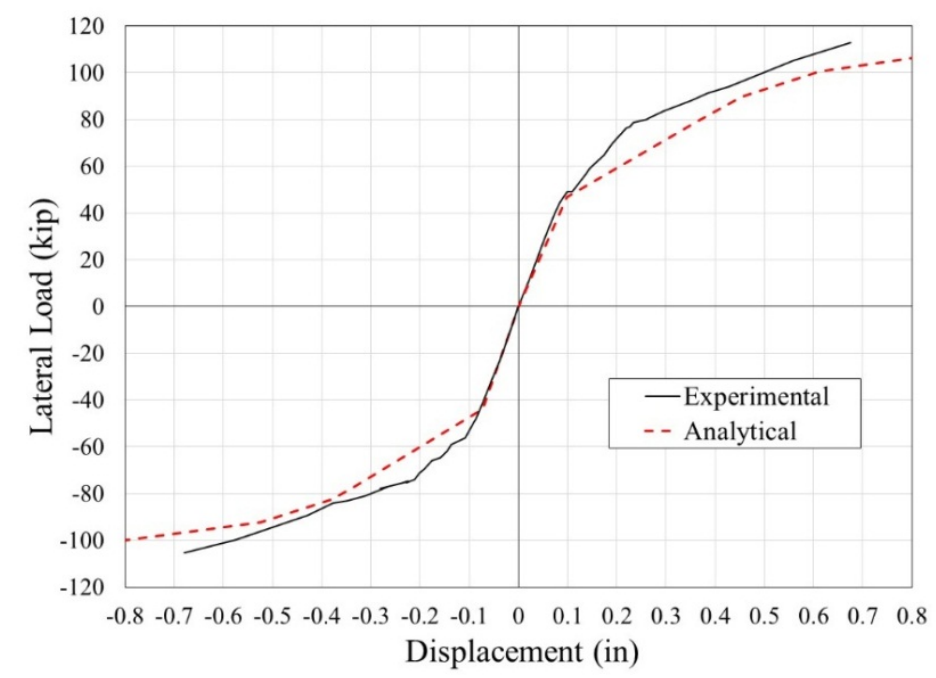

Figure 7.28: Experimental vs. analytical envelope curves for Model 1

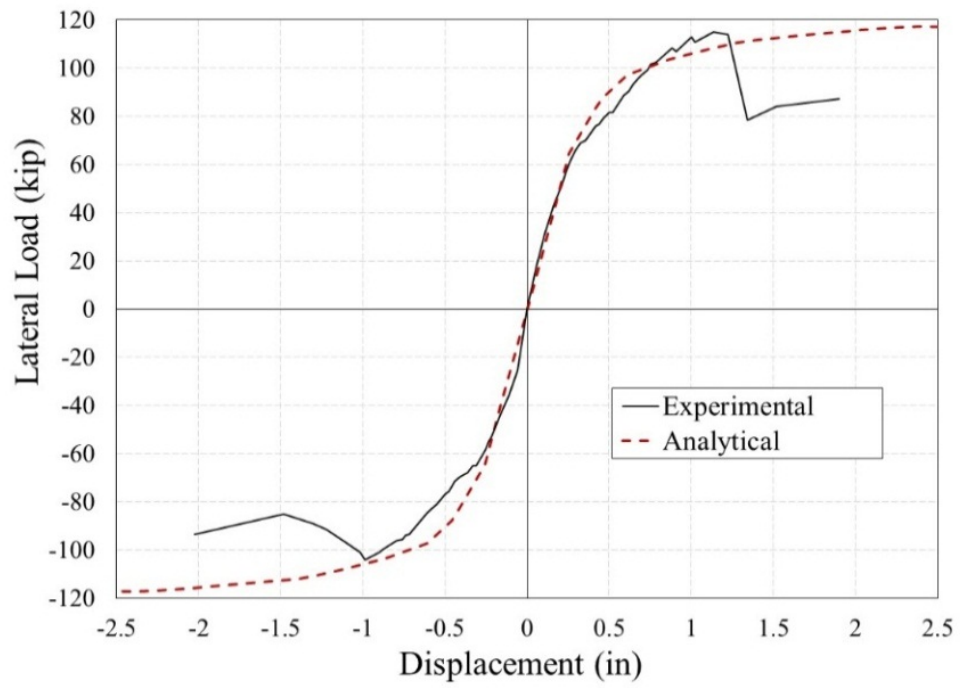

Figure 7.29: Experimental vs. analytical envelope curves for Model 2 


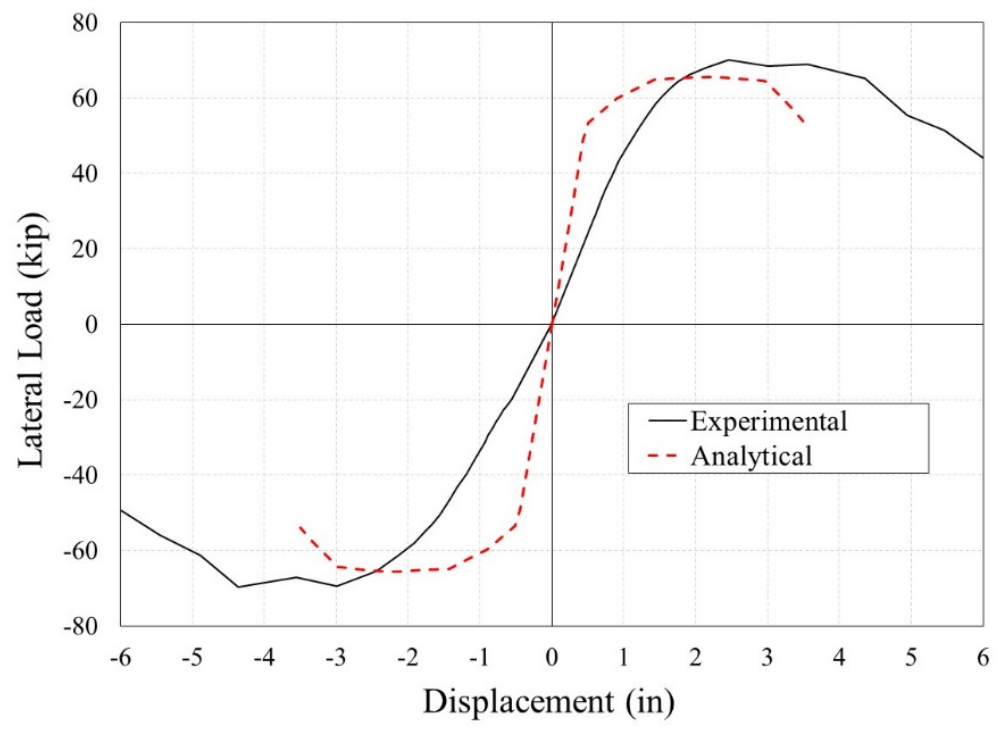

Figure 7.30: Experimental vs. analytical envelope curves for the As-built bent

\subsubsection{Comparison between backbone curves}

Figure 7.31 shows a comparison between the backbone curves for the three specimens tested. The Model 1 and 2 showed greater strength and stiffness as compared to the Asbuilt bent. This behavior was expected because the inclusion of the BRB, which acts in parallel with the RC bent, adds stiffness to the overall system as depicted in Figure 6.9. The fact that Model $1 \mathrm{had}$ an abrupt drop in load was attributed to the poor confinement of the brace.

Despite the seismic detailing deficiencies, the backbone curve for the As-built bent showed moderate displacement capacity. This behavior is likely a result of a relatively long lap splice length $\left(40 \mathrm{~d}_{\mathrm{b}}\right)$ and a low axial load level $\left(0.1 \mathrm{f}_{\mathrm{c}} \mathrm{A}_{\mathrm{g}}\right)$. Even though this behavior was unexpected because the vast number of deficiencies of the as-built bent, similar results can be found in the literature (McLean et al. 1998), (Pantelides and Gergely 2008). 


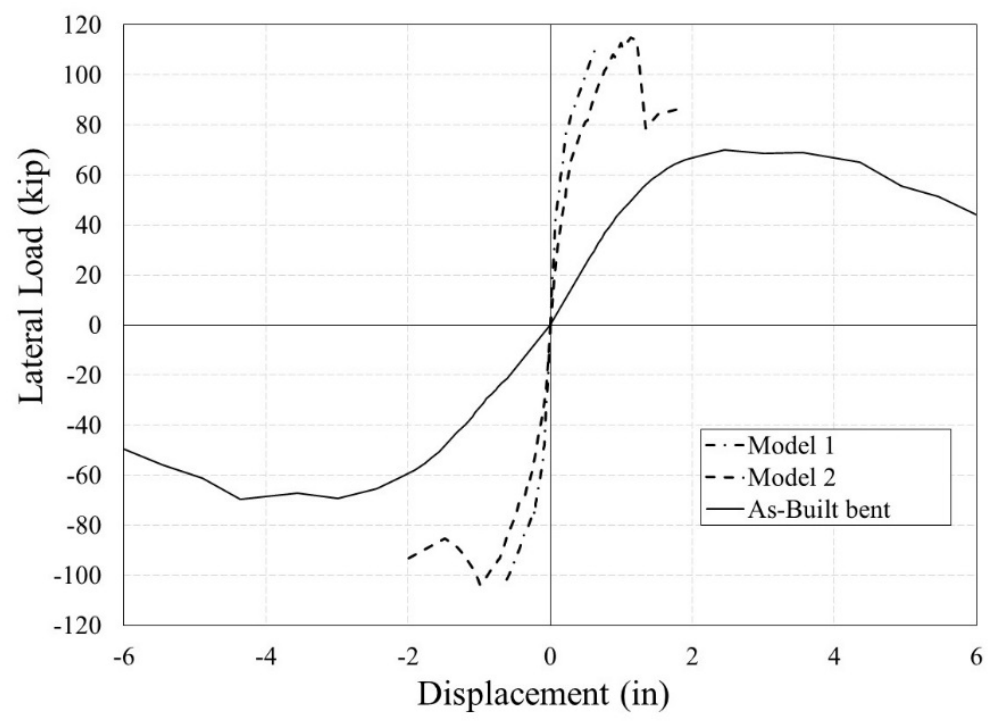

Figure 7.31: Backbone response comparison

\subsubsection{Stiffness degradation}

The stiffness degradation is an important property in RC bridges subjected to seismic events since it changes the effective natural period of the structure. Stiffness degradation can be attributed to brace nonlinear behavior, concrete nonlinear behavior caused by flexural and shear cracking, slippage of steel reinforcement, etc.

In this study, the normalized stiffness, represented as the secant stiffness divided by the yield stiffness, at different displacement ductilities, was utilized as the stiffness degradation parameter. It is worth noting that for Models 1 and 2 the yield displacement used in the calculations corresponds to the yield displacement of the BRB. On the other hand, the normalized stiffness degradation for the As-built bent was not calculated since its yield stiffness was already deteriorated. This stiffness deterioration occurred when the bent was previously tested in a retrofitted state.

In terms of stiffness degradation both test specimens showed similar stiffness degradation up to a displacement ductility 2. However, for ductilities above 2, the Model 1 showed a lower stiffness degradation than Model 2. This is concordant with the hysteretic response showed by the two specimens, in which Model 1 showed a stable response throughout the test. On the other hand, the stiffness degradation in the negative direction for Model 2 showed a decay at a displacement ductility of 3.2, which is consistent with the strength degradation shown in Figure 7.19. Moreover, Model 2 showed an abrupt decay at a displacement ductility of 4.2 due to rupture of the BRB steel core.

Gulkan and Sozen (Gulkan and Sozen 1974) proposed a relation between the displacement ductility $(\mu)$ and the secant stiffness $\left(\mathrm{k}_{\mathrm{sec}}\right)$ as shown in Eq. (4.1). That relation does not agree well with the stiffness degradation for Models 1 and 2 since it was developed to be representative of deficient columns. Further, this result implies that the retrofitted bent has lower rate of stiffness degradation than deficient RC components. 


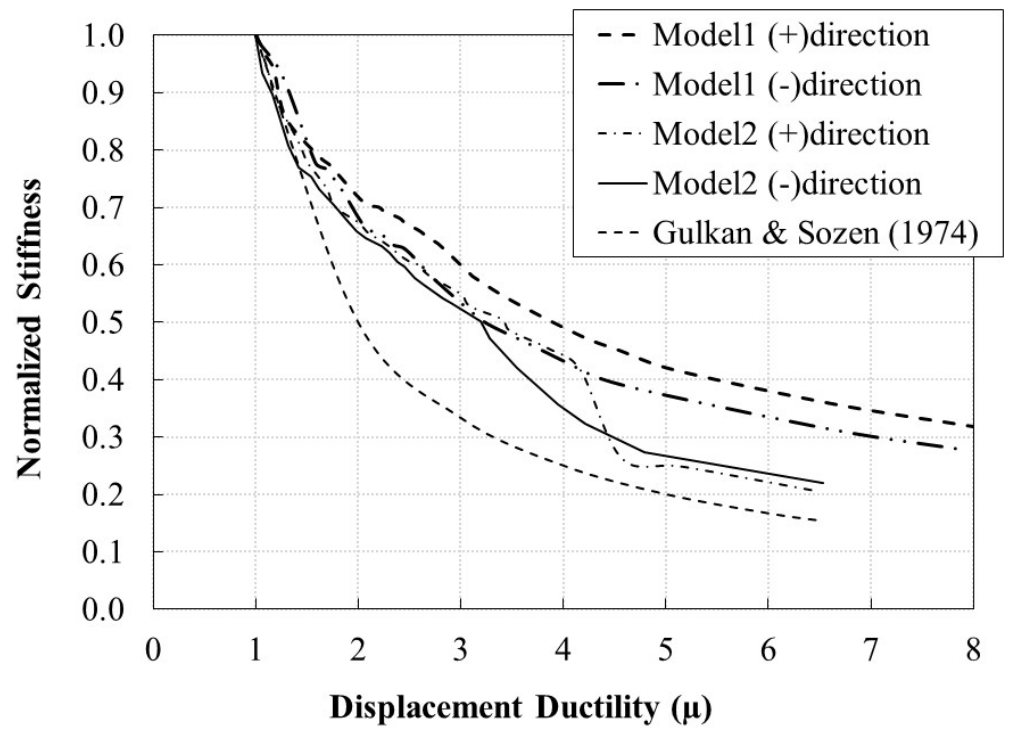

Figure 7.32: Stiffness degradation Model $1 \& 2$

\subsubsection{Energy Dissipation and Equivalent Viscous Damping}

The property of dissipating energy through hysteretic behavior is desirable in structures subjected to major seismic events. In this study the amount of energy that was dissipated by the specimens was calculated as the area $\left(A_{d}\right)$ enclosed by a full cycle as shown in Figure 7.33 .

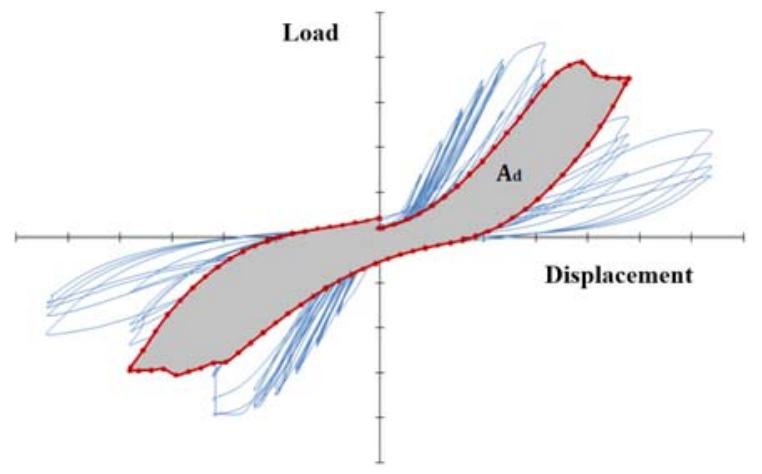

Figure 7.33: Illustration of energy dissipated through hysteretic behavior.

A central task in any modern seismic analysis method is to determine the equivalent viscous damping of a structure. This is especially important in bridges that are designed and retrofitted using displacement based analysis. Thus, an important parameter in any experimental program is to determine the equivalent viscous damping of the structure.

The total equivalent viscous damping equation is divided in two expressions:

$$
\xi_{e q}=\xi_{e l}+\xi_{\text {hyst }}
$$


Where, $\xi_{\text {el }}$ denotes the initial elastic damping, which is typically considered as $5 \%$ for RC structures, and $\xi_{\text {hys }}$ denotes the hysteretic damping.

The hysteretic damping can be calculated by using Eq. (7.5). (Priestley et al. 2006)

$$
\xi_{\text {hys }}=\frac{1}{4 \pi} \frac{A_{d}}{A_{\text {strain }}}=\frac{1}{2 \pi} \frac{A_{d}}{F_{\text {max,cycle }} \cdot \delta_{\text {max }, \text { cycle }}}
$$

Where, Ad is the energy dissipated in a full cycle as shown in Figure 7.33 and $\mathrm{A}_{\text {strain }}$ is the strain energy (or stored energy) measure at the peak force of each cycle.

The equivalent viscous damping $\left(\xi_{\mathrm{eq}}\right)$ was calculated for each specimen and is illustrated in Figure 7.34. The displacement ductility $(\mu)$ used in this section for Models 1 and 2 was computed in terms of the yield displacement of the bent $\left(\delta_{\mathrm{y}}^{\mathrm{B}}\right)$ instead of the yield displacement of the BRB $\left(\delta_{\mathrm{y}}{ }^{\mathrm{BRB}}\right)$ in an effort to allow comparisons between the equivalent viscous damping results of each test specimen.

The results showed that Model 1 has the greatest equivalent viscous damping $\left(\xi_{\text {eq }} \approx 27 \%\right)$ as compared to Model 2 and the As-built specimens. This result is consistent with the stable hysteretic behavior and wide loops shown by Model 1. As shown in Figure 7.34, the equivalent viscous damping $\left(\xi_{\mathrm{eq}}\right)$ increased with increasing levels of displacement ductility. However, once the specimen failed it can be seen two trends. For the As-built specimen the $\xi_{\text {eq }}$ remained constant with increasing ductility, on the contrary, in Model 2 the equivalent viscous damping decreased with increasing ductility following a similar trend to the strength degradation shown in Figure 7.19 for the load-displacement response. It is worth noting the fact that all the specimens had $\xi_{\text {eq }}$ values of approximately $25 \%$.

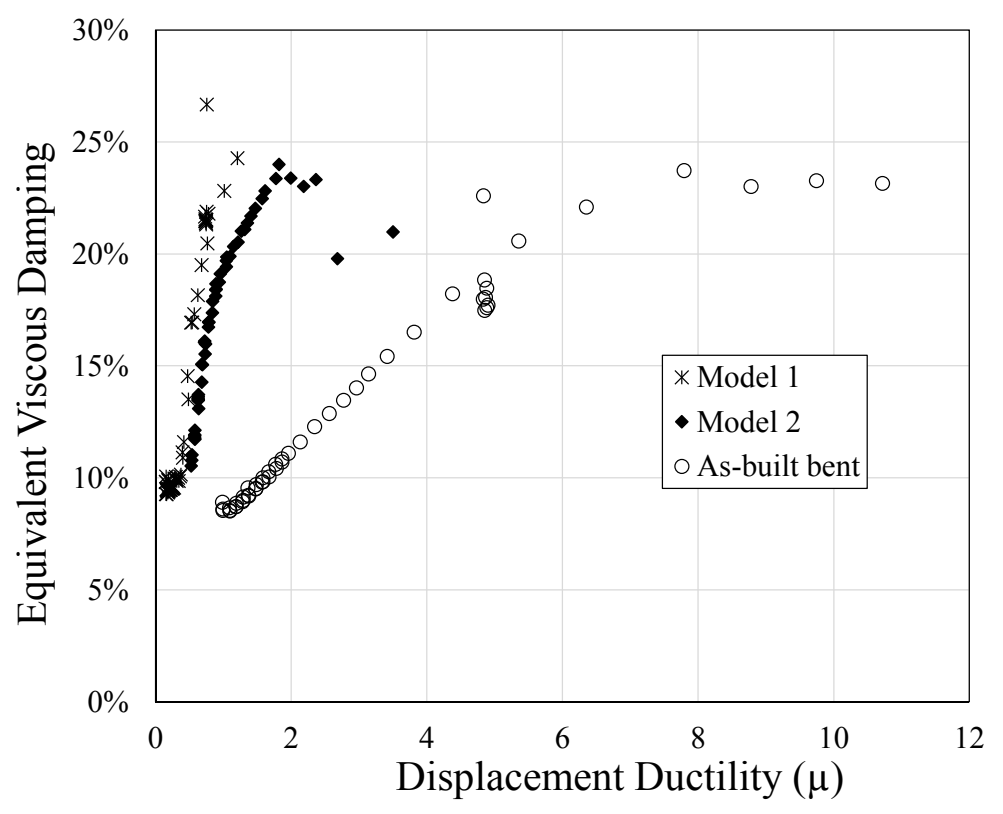

Figure 7.34: Equivalent viscous damping. 


\subsection{SUMMARY}

A case study of a representative reinforced concrete bridge bent constructed in the 1950 to mid1970 in the Pacific Northwest was presented in an effort to assess its deficiencies and demonstrate the retrofit procedure of adding BRBs for the transverse direction. Retrofitted and unretrofitted cases were tested using cyclic loading protocols representative of the displacement demands in RC bridge bents subjected to subduction zone earthquakes. The retrofitted RC bridge bent was designed to perform elastically or with minor inelastic excursions within the original bent throughout the different seismic hazard design levels. Two BRB designs were considered in this study in an effort to assess the influence of BRB stiffness on the overall structural performance. A novel gusset plate to $\mathrm{RC}$ bent connection was used, in which the gusset plates were directly connected to the horizontal RC elements without interfering with the columns.

Observations based on numerical and experimental results can be summarized as follows:

- The numerical results showed that by implementing buckling restrained braces, the retrofitted bent was significantly stiffer than conventionally retrofitted and yet provided for ductile response without significant damage to the concrete elements and could be a suitable retrofit measure for successfully achieving performance based dual-level design approaches. Even though, the proposed design implementation was developed for a diagonal configuration in mind, its application can be extended to other cases.

- The results of these large-scale experiments successfully demonstrated the effectiveness of utilizing buckling restrained braces for achieving high displacement ductility of the retrofitted structure, while also controlling the damage of the existing vulnerable reinforced concrete bent up to the design performance levels. No damage was observed in the connection regions of the brace throughout the loading history, leaving the potential for replaceability of the sacrificial BRB element. The potential for improving the overall seismic behavior and the design performance levels with BRBs offers bridge design professionals a viable method for performance driven retrofit of multi-column reinforced concrete bridge bents.

- The results also indicated that despite the detailing deficiencies of the multi-column RC bridge bents built before 1970 in the Pacific Northwest, the cyclic response of the unretrofitted bent exhibited moderately ductile performance. The moderately ductile performance is likely a result of a relatively long lap splice length $\left(40 \mathrm{~d}_{\mathrm{b}}\right)$, and low axial column loads $\left(0.1 \mathrm{f}^{\prime} \mathrm{c} \mathrm{Ag}\right)$. 


\subsection{FRAGILITY CURVES}

\subsection{GENERAL}

The economic impact that a severe earthquake has on a highway network has led governmental entities and research groups to develop reliable methods for the damage assessment of components and highway structures in general. One of these methodologies is to base the damage assessment in fragility curves. Bridge damage fragility curves describe the conditional probability of exceeding a level of direct or indirect bridge damage for a given level of seismic hazard. Nowadays, fragility curves have emerged as an important decision tool to prioritize bridge retrofitting and estimate potential losses during and after a major earthquake.

In this chapter a brief state of the art in bridge fragility curve development is presented, limitations of each fragility development method are discussed, and fragility curves for a representative bridge bent in its as built and retrofitted conditions are developed by using analytical methods. In order to develop those fragility curves actual subduction zone strong motion records in conjunction with nonlinear static analysis performed in SAP2000 were used.

\subsection{SEISMIC FRAGILITY}

Seismic fragility analysis was born as a consequence of lifeline earthquake engineering. Current highway structure design methodologies are trending to performance-based design, in which fragility curves play an important role of describing the performance of a structure or component at different levels of a selected seismic intensity measure (Mackie and Stojadinovic 2005).

The fragility of a structure or component can be expressed as a conditional probability that a defined limit state (LS) is exceeded for a given level of ground motion intensity (IM), as follows:

$$
\text { Fragility }=P[L S \mid I M]
$$

\subsection{FRAGILITY DEVELOPMENT METHODS}

The development of fragility cures has been widely discussed in the literature (Shinozuka et al. 2000), (Stojadinović and Mackie 2007), (Vosooghi and Saiid Saiidi 2012) and several methods have been applied for its development. Fragility development methods can be categorized in empirical methods, experimental methods, expert opinions, and analytical methods. A detailed literature review of fragility curves development methods can be found in Mehary and Dusicka (Mehary and Dusicka 2015), and in Muntasir Billah and Alam (Muntasir Billah and Alam 2014).

\subsubsection{Empirical Methods}

Empirical fragility curves are developed by utilizing observed damage data during past earthquakes. These damage data was collected primarily during the 1971 San Fernando 
earthquake, 1989 Loma Prieta earthquake, and the 1994 Northridge earthquake, which showed the high vulnerability of deficient bridges subjected to strong ground motions.

Empirical fragility curve development is relatively straightforward since earthquake damage reports are used to establish the relationship between the ground motion intensity and the damage state of each bridge.

Several reasearchers have conducted studies to develop bridge fragility curves by statistically analyzing empirical damage data from damage reports (Basoz and Kiremidjian 1998), (Yamazaki et al. 1999), (Shinozuka et al. 2000).

Despite empirical methods may represent a more realistic approach due to the use of actual damage reports, they lack generality and have a large degree of uncertainty, which is primarily due to potential discrepancies in damage observation between inspection teams.

\subsubsection{Expert Opinion}

In this method the opinion of experts is collected and analyzed to estimate structural damages. The expert opinion is usually considered through surveys, which are then analyzed and represented in a damage probability matrix describing damage state for different levels of ground motion intensity. Finally, the probability matrix generated from the survey results is used for developing the fragility curves (ATC 1991), (Grossi 2000).

The expert opinion is the only source for the development of fragility curves. As a result, this method depends on the experience of the engineer and the number of expert's opinion gathered, which can lead to largely bias fragility curves and question its reliability.

\subsubsection{Experimental Methodology}

In this method the results from large-scale or full-scale experiments are needed for the development of fragility curves. Vosooghi and Saiidi (Vosooghi and Saiidi 2012) developed experimental fragility curves for reinforced concrete bridge columns based on data from 32 bridge column models tested on shake tables. However, a lack of data at the same damage state is evident due to the limited amount of large scale testing. This lack of data highly limits the application of the experimental fragility curves.

\subsubsection{Analytical Methods}

\subsubsection{Elastic spectral analysis}

In this method the capacity/demand ratio of different components are determined to evaluate their potential seismic damage. In order to develop fragility curves using this method, damage states are defined and correlated to the capacity/demand ratio of the component via statistical analysis. The capacity/demand ratios are obtained using elastic spectral analysis (Hwang et al. 1999) (Hwang et al. 2000) (Hwang et al. 2001). 
Despite the easy implementation of this method, its limitation is apparent at not be able to account for nonlinear behavior.

\subsubsection{Non-linear static analysis (NSA)}

Nonlinear static analysis (NSA), also called Pushover analysis, is used in conjuntion with probabilistic analysis to determine fragility curves. (Mander and Basöz 1999), (Shinozuka et al. 2000), (Liao and Loh 2004).

In this method uncertainty in capacity and demand is considered by plotting log-normal distributions over the capacity and demand curves. For a particular intensity measure level the probability of failure can be estimated by using the intersection of capacity and demand distribution as shown in Figure 8.1. Finally, the fragility curves can be generated by increasing the level of intensity measure (IM) and measuring the response at various damage states (DS).

Shinozuka et al. (Shinozuka et al. 2000) examined fragility curves of a bridge by time history analysis and the capacity spectrum method which is one of the nonlinear static procedures developed for buildings. Their comparison of fragility curves generated by the nonlinear static procedure with those by time-history analysis indicated that there was good agreement for the state of minor damage, but not as good for the state of major damage where nonlinear effects played an important role. However, they also concluded that even for the state of major state the agreement between the fragility curves based on NSA and nonlinear time history analysis was adequate considering the large number of assumptions that are performed for obtaining the fragility curves.

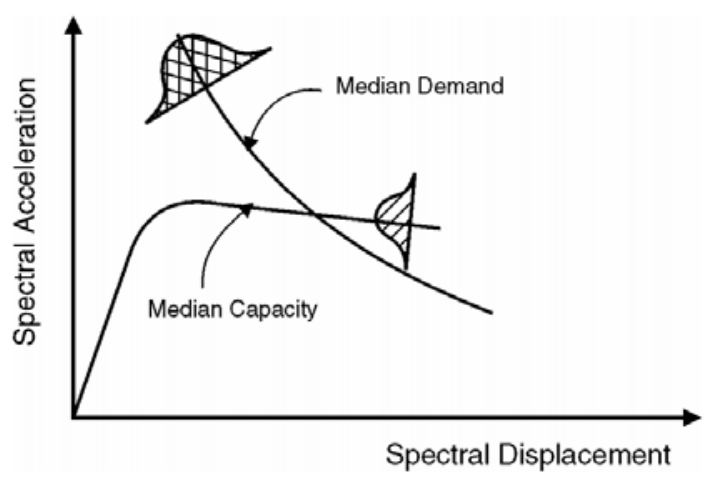

Figure 8.1: Capacity vs demand spectra showing uncertainty in structural behavior and ground motion response (FHWA 2006).

This method overcomes the deficiencies of performing an elastic spectral analysis by considering nonlinearities. However, in this method not all nonlinear effects are considered, which limits its application. 


\subsubsection{Non-linear time history analysis}

Nonlinear time histories are used to construct analytical fragility curves.. In this method ground motion time histories are selected to represent the seismic hazard at a specific area of interest. Nonlinear time histories analysis are conducted to obtain the response of the structure in study. Damage states and intensity measures are defined to quantify the damage undergo for the structure. Probabilistic analysis of the median and log-standard deviation parameters are then estimated by maximum likelihood procedure and the fragility curves are generated. (Shinozuka et al. 2000), (Hwang et al. 2001), (Shinozuka et al. 2001), (DesRoches et al. 2003), (Pan 2007), (Nielson and DesRoches 2007), (Simon et al. 2010).

Despite of being the most computationally demanding and time consuming, this method is the most reliable one for generating fragility curves.

\subsubsection{Fragility Curves for Retrofitted Bridges}

Currently, limited bridge fragility has been developed for retrofitted bridges. Some of this researches are summarized below.

Shinozuka et al. (Shinozuka et al. 2002) developed fragility curves for two retrofitted bridges by means of steel jacketing of columns. In order to develop the fragility curves, they used SAP2000 for modeling the bridges and sixty ground motion recordings representative of Los Angeles.

Padgett (Padgett 2005) reviewed bridge seismic retrofit practice in the Central and Southeastern United States. Padgett used 48 strong ground motions as a seismic hazard and the Open System for Earthquake Engineering Simulation (OpenSees) computational analysis program to model the bridges. Non-linear time history analysis were conducted to capture parameters of interest for each component. The fragility was modeled by a lognormal cumulative distribution function where the structural demand and capacity were assumed to be lognormal distributed.

Padgett and DesRoches (Padgett and DesRoches 2007, 2008, 2009 presented an analytical methodology for developing fragility curves for retrofitted bridge systems for the Central and Southeastern U.S. Since there were no records of strong motions in that area, they used two suites of synthetic ground motions for the study.

Muntasir Billah et al. (Muntasir Billah et al. 2012) developed fragility curves for retrofitted multicolumn bridge bents subjected to near-fault and far field ground motions using a probabilistic seismic demand model and incremental dynamic analyses (IDA). The retrofit measures considered in that study were concrete jacketing, steel jacketing, carbon fiber reinforced polymer (CFRP) jacketing, and cementitious composite jacket. The results, as anticipated, showed that the bent retrofitted with concrete jacketing was more vulnerable to seismic ground motions. On the contrary, the bent retrofitted with CFRP and cementitious composite showed less vulnerability under both far-field and near-fault earthquakes. 


\subsection{FRACILITY CURVES FOR REPRESENTATIVE BRIDGE BENT}

In the current study, the nonlinear static analytical method is used to evaluate the seismic fragility of the representative bridge bent built prior 1970 in the State of Oregon, as described in Section 7.2, and its retrofitted state with buckling restrained braces. A two-dimensional (2D) finite-element model scheme modeled in SAP2000 (shown in Figure 7.25) was used in the analytical modeling because of the good agreement between experimental and analytical results as depicted in Figure 7.26, and Figure 7.28 for the retrofitted and as-built condition, respectively. Moreover, for simplicity, the bridge bent is assumed to be supported by rigid foundations.

The methodology for fragility curve development used in this study follows the one proposed by Shinozuka et al. (Shinozuka et al. 2000), which is based on Capacity Spectrum method as a nonlinear static procedure.

\subsubsection{Strong Ground Motions}

A total of 30 earthquake ground motions, of which 10 are from the Tohoku earthquake M 9.0, 10 from the Maule, Chile earthquake M 8.8, and 10 from the Valparaiso, Chile earthquake M 7.8 were utilized to evaluate the likelihood of exceeding the seismic capacity of the bridge bent in an effort to represent a potential Cascadia earthquake scenario. The seismic actions were represented by means of 5\% damped elastic response spectra as shown in Figure 8.2.

\subsubsection{Damage States}

Damage states (DS) for a bridge component should provide a useful qualitative and quantitative representation for that component. These DS are often based on visual representations or strain and stress levels obtained from experimental studies. In this work, the engineering demand parameter (EDP) used for measuring the damage state of the bridge bent was displacement ductility, $\mu$. The EDP values are based on available literature (Hwang et al. 2001) and are shown in Table 8.1. The displacement ductility for the damage state of collapse was adapted to the maximum ductility of the representative bridge bent.

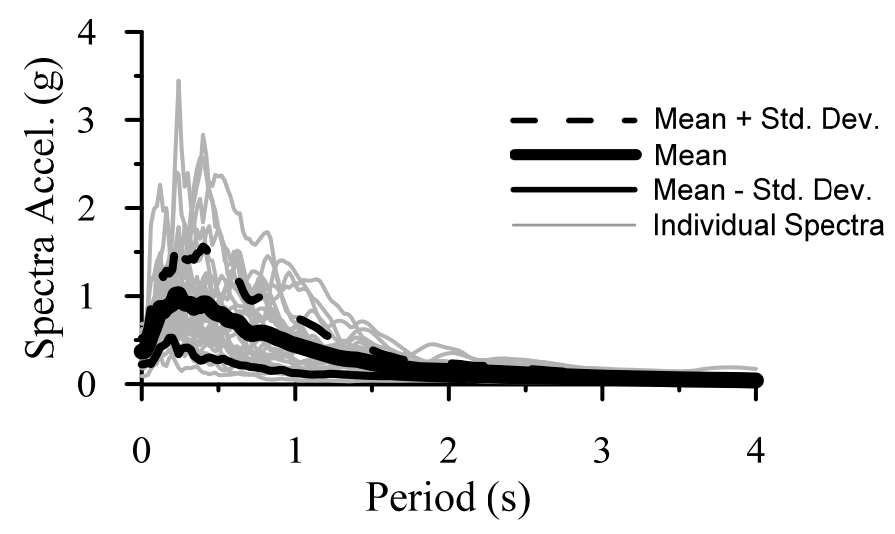

Figure 8.2: Response spectra for the 30 time histories (5\%damping) 
Table 8.1: Damage states for fragility curve development

\begin{tabular}{c|c|c|c|c}
\hline \multirow{2}{*}{ EDP } & \multicolumn{4}{|c}{ Damage States } \\
\cline { 2 - 5 } & Slight & Moderate & Extensive & Collapse \\
\hline Displacement ductility & $\mu>1$ & $\mu>1.2$ & $\mu>1.76$ & $\mu>3.5$ \\
\hline
\end{tabular}

\subsubsection{Fragility Curves}

Nonlinear static analyses were performed in SAP2000 by using characteristic material properties for reinforcing steel and concrete, and the model described in Section 7.3.4.2.

For a given damage state, the fragility curve defines the conditional probability that the damage state be equaled or exceeded as a function of an intensity measure (IM). Following the work carried out by Shinozuka et al. (Shinozuka et al. 2000), the intensity measure considered in this study was the peak ground acceleration (PGA). The strong ground motion time histories were scaled to specific PGAs. The PGA ranged from $0.05 \mathrm{~g}$ to $0.8 \mathrm{~g}$ in increments of $0.1 \mathrm{~g}$. (e.g. 0.05, $0.1,0.2 \ldots 0.8 \mathrm{~g}$ ). For each group of PGA the mean and the mean $\pm 1 \sigma$ (standard deviation) was calculated. This process generated three elastic acceleration response spectra, and consequently three spectral displacements were determined by intersecting the capacity spectrum with the demand spectrum. The displacement demands determined from this process are depicted in Figure 8.3 and Figure 8.4 for the as-built RC bridge bent and retrofitted bent, respectively. Since the distribution of spectral displacement was not symmetric, different standard deviations ( $\sigma^{+}$ and $\sigma$-) were determined.

In this study the displacement ductility was determined by dividing the spectral displacement by the equivalent spectral displacement at yield. Thus, the displacement ductility has mean $\overline{\mu_{d}}$ and standard deviation, $\sigma$, redefined as $\sqrt{\sigma^{+} \cdot \sigma^{-}}$.

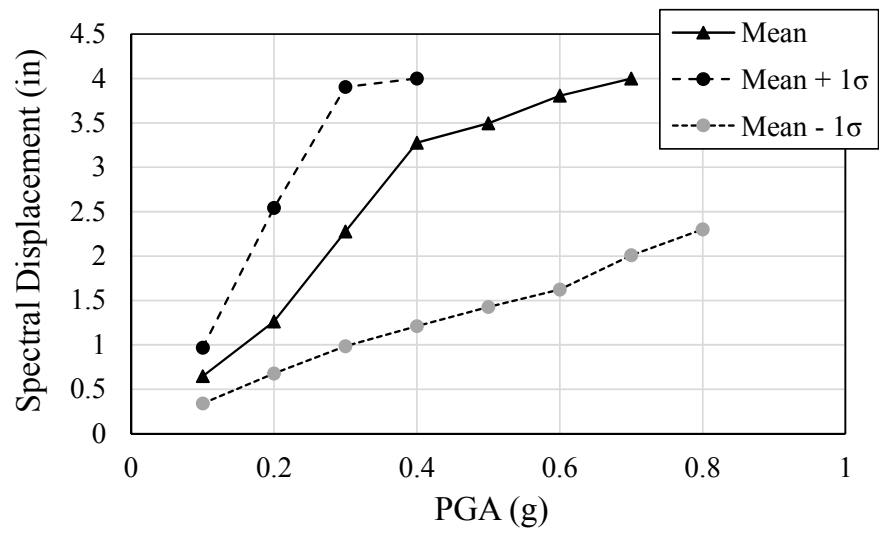

Figure 8.3: Performance displacement of as-built RC bridge bent 


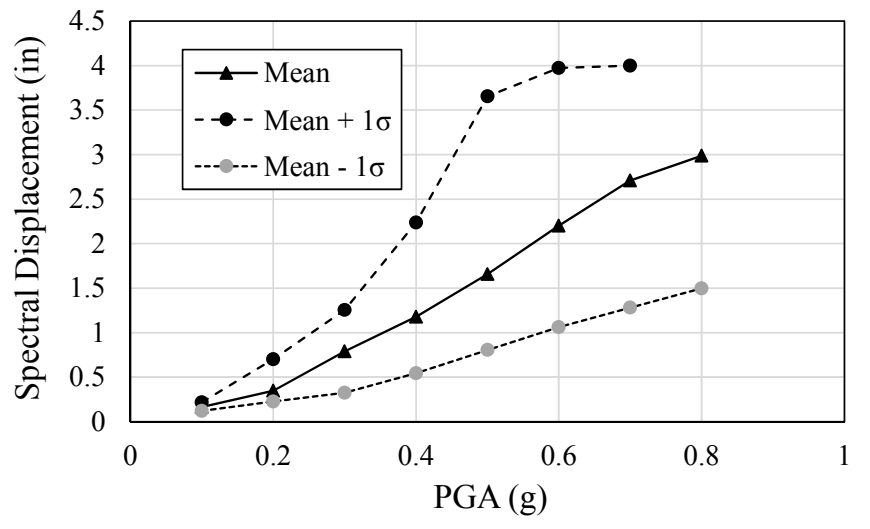

Figure 8.4: Performance displacement of retrofitted RC bridge bent

The following equation defines the fragility curve.

$$
P\left[\mu_{d} \geq \mu \mid P G A\right]=1-\Phi\left[\frac{1}{\xi} \ln \left(\frac{\mu}{c}\right)\right]
$$

Where, $\Phi$ is the standardized normal distribution function, $\mu_{\mathrm{d}}$ is the displacement demand ductility, $\mu$ is the displacement demand capacity shown in Table $8.1, \xi$ and $\mathrm{c}$ are the standard deviations and the mean values of the corresponding normal distribution. As proposed by Shinozuka et al. (Shinozuka et al. 2000), the standard deviation $\xi$ and the mean $c$ at each PGA can be obtained from Eq. (8.3) and (8.4)

$$
\begin{gathered}
\overline{\mu_{d}}=c \cdot \exp \left[\frac{\xi^{2}}{2}\right] \\
\sigma^{2}=\left\{\overline{\mu_{d}}\right\}^{2}\left[\exp \left(\{\xi\}^{2}\right)-1\right]
\end{gathered}
$$

The probability of exceedance for the selected damage states is depicted in Figure 8.5 and Figure 8.7 for the as-built RC bent and its retrofitted condition, respectively. It is worth noting, that the displacement ductility for the retrofitted case was performed with respect to the yield displacement of the as-built bent instead of the yield displacement of the BRB.

Median values and dispersion for each damage state are shown in Table 8.2 and Table 8.3 for the as-built and retrofitted bent, respectively. Comparison of the median values for the as-built state to the retrofitted state is shown in Figure 8.9.

As can be observed in the figures, the retrofitted state presents a reduced probability of damage for all the damage states as compared to the as-built condition. Moreover, the median PGA value, which represents a probability of exceedance of $50 \%$, for the retrofitted bent is more than twice than the one for the as-built bent. This result implies, as expected, the retrofitted bridge bent is less vulnerable than the as-built bent. 


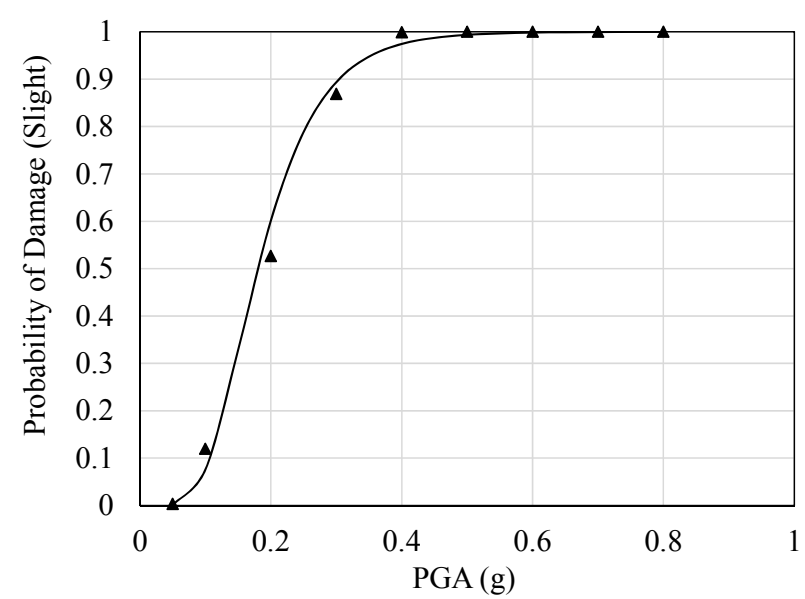

(a)

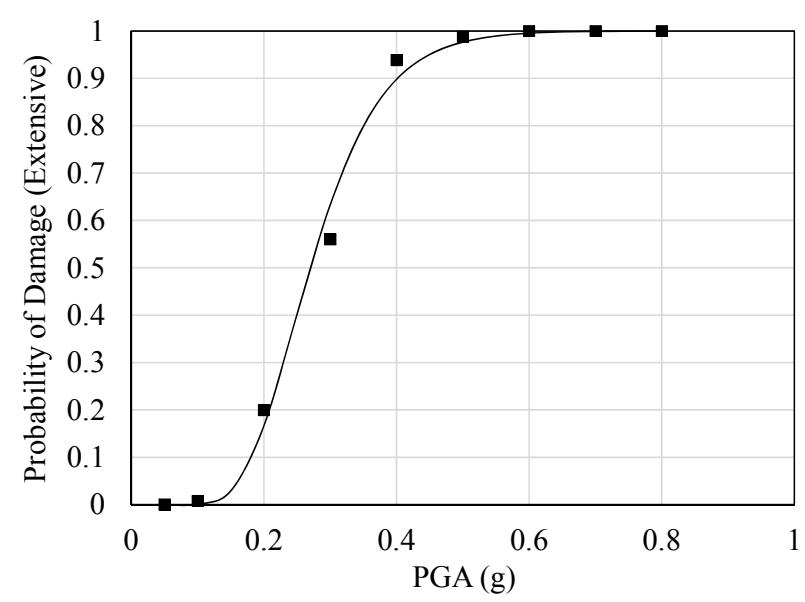

(c)

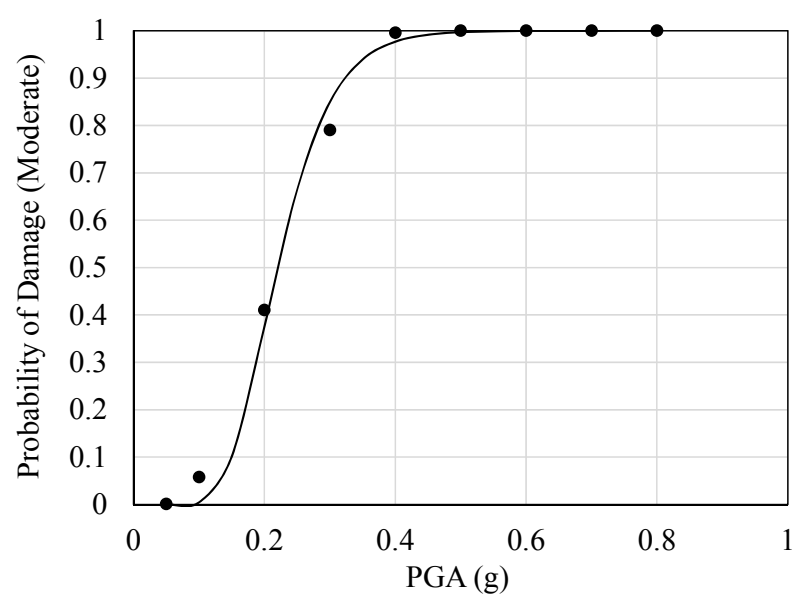

(b)

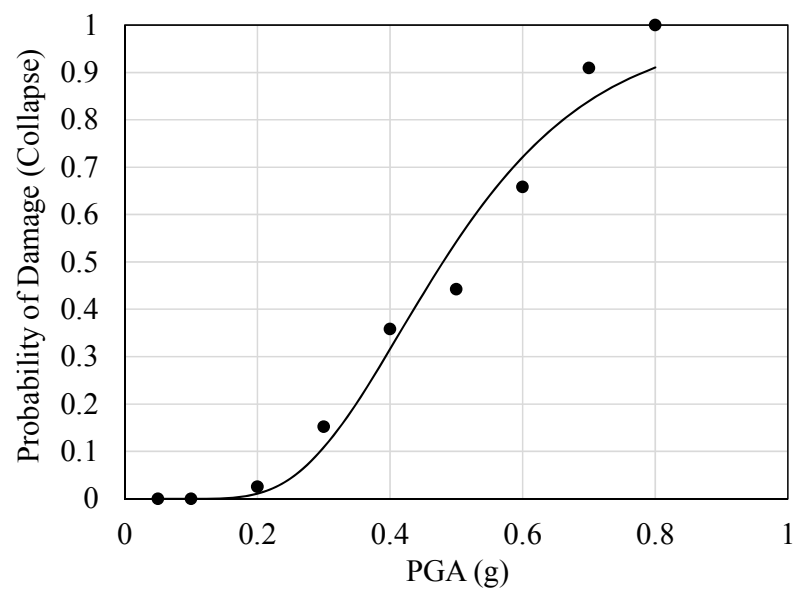

(d)

Figure 8.5: Fragility curves for representative as-built RC bridge bent for damage state: (a) Slight, (b) Moderate, (c) Extensive, (d) Collapse.

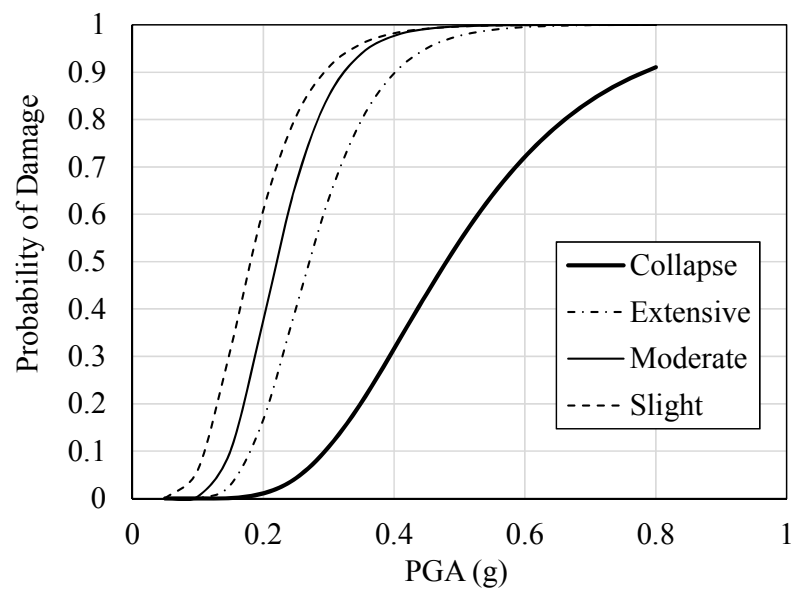

Figure 8.6: Fragility curves for representative as-built RC bridge bent. 


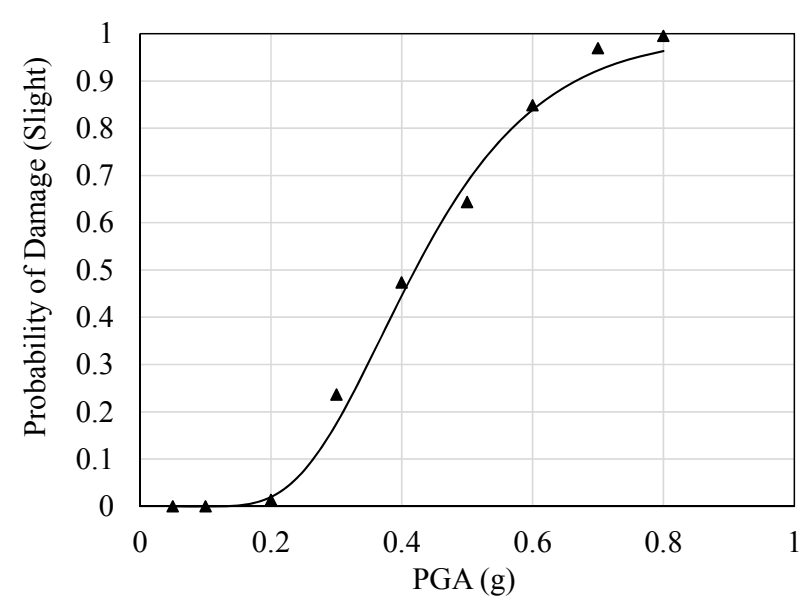

(a)

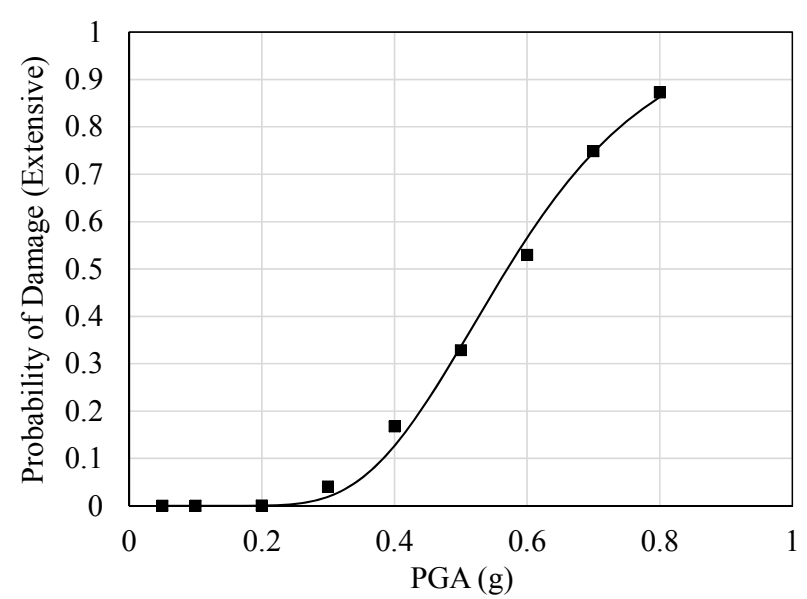

(a)

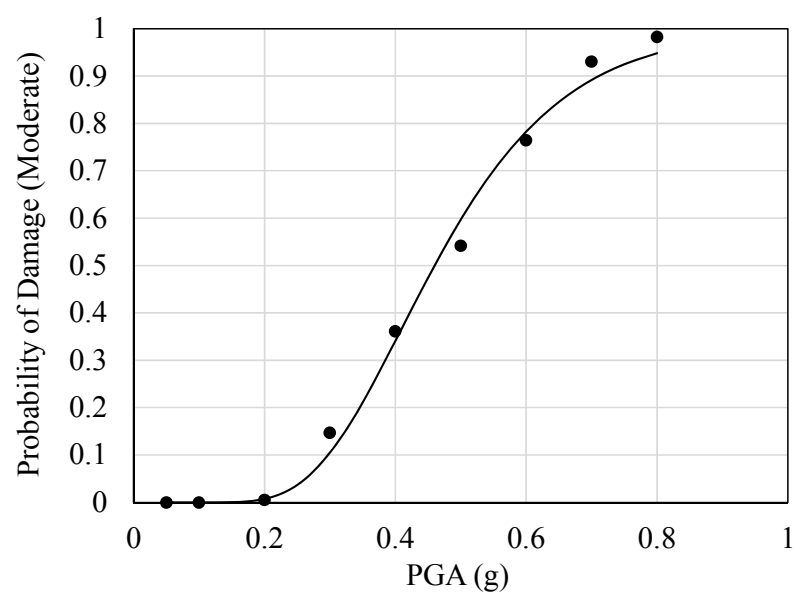

(b)

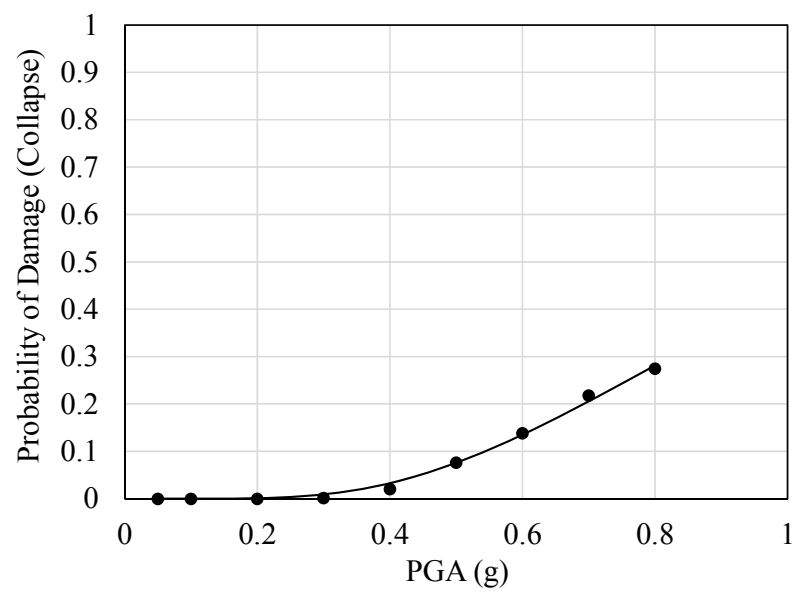

(b)

Figure 8.7: Fragility curves for retrofitted RC bridge bent (deterministic) for damage state: (a) Slight, (b) Moderate, (c) Extensive, (d) Collapse. 


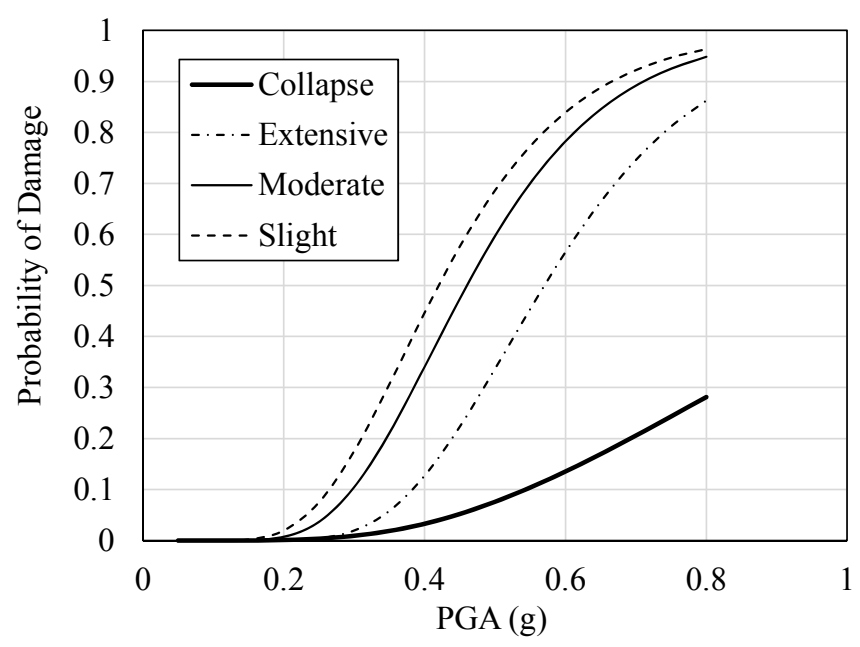

Figure 8.8: Fragility curves for retrofitted RC bridge bent.

Table 8.2: Fragility curves values for representative RC bridge bent

\begin{tabular}{c|c|c|c|c}
\hline \multirow{2}{*}{ Parameter } & \multicolumn{4}{|c}{ Damage States } \\
\cline { 2 - 5 } & Slight & Moderate & Extensive & Collapse \\
\hline Median & 0.18 & 0.22 & 0.27 & 0.48 \\
\hline Dispersion & 0.38 & 0.30 & 0.31 & 0.38 \\
\hline
\end{tabular}

Table 8.3: Fragility curves values for retrofitted $\mathrm{RC}$ bridge bent

\begin{tabular}{c|c|c|c|c}
\hline \multirow{2}{*}{ Parameter } & \multicolumn{4}{|c}{ Damage States } \\
\cline { 2 - 5 } & Slight & Moderate & Extensive & Collapse \\
\hline Median & 0.42 & 0.46 & 0.57 & 1.1 \\
\hline Dispersion & 0.36 & 0.34 & 0.31 & 0.55 \\
\hline
\end{tabular}

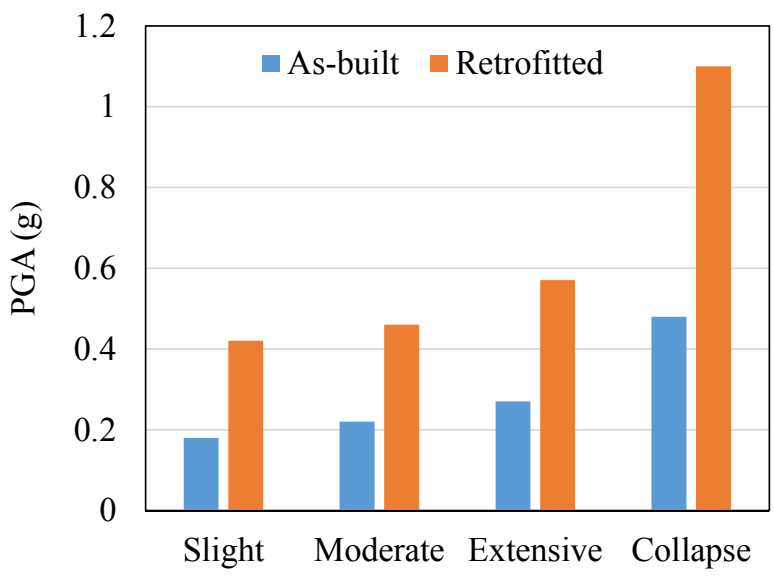

Figure 8.9: Comparison of median values of PGA for representative RC bridge bent 


\subsection{SUMMARY}

Fragility curves for a seismically deficient RC bridge bent and the bent retrofitted with buckling restrained braces were developed. The methodology used for the development of the fragility curves involved the use of 30 subduction zone ground motions and the use of 2D nonlinear analytical models. The analytical method used in the study was the nonlinear static analysis (pushover) following the study carried out by Shinozuka et al. (Shinozuka 2000). This method was selected due to its simplicity and the good agreement between the experimental results shown in a previous chapter and the analytical results computed by means of pushover analysis. Through the process, the impact of the retrofit measure and the vulnerability of the as built bridge bent was evaluated.

The numerical results showed that the representative as-built bridge bent is more susceptible to subduction zone ground motions as compared to the response of the RC bent retrofitted with buckling restrained braces. As a result, the analyses of the fragility curves revealed the effectiveness of the proposed retrofit measure in mitigating probable damage undergo by the structure during a major seismic event. The fragility curves and the retrofit measure as presented in this study aim to improve the criteria for retrofitting prioritization, estimation of potential losses and help with the decision of selecting a suitable retrofit measure in areas prone to subduction zone mega earthquakes.

The authors recognize the need for more research in the assessment of fragility by further studying the use of different bridge bent models with variable geometry and material properties. 


\subsection{CONCLUSIONS}

\subsection{CONCLUSION}

The analysis of strong ground motions led to the conclusion that for all short and medium period structures $(\mathrm{T}<1.0 \mathrm{~s})$, subduction earthquakes produced more cumulative displacement demands than crustal earthquakes. For long period structures $(T>1.0 \mathrm{~s})$ the results showed that the characteristics of the ground motion and fault itself affect the cumulative demand more than magnitude or acceleration content. Moreover, the records from the Maule earthquake produced always a $200 \%$ more demand that the crustal ground motions used in the study. Other measures of demand such as, acceleration spectra, duration of strong shaking, and residual displacement suggested that the Tohoku 1 ground motion set, which had the higher PGA values, would produce the highest demand.

Three hysteretic behaviors, namely, bilinear, elastic non-linear and degrading were analyzed. A bilinear model was utilized to represent steel components behavior, an elastic non-linear model was included to represent structures that display rocking behavior during earthquakes, as the benefits of utilizing rocking motion in structures are becoming more widely acknowledged and understood, and a deterioration model was included to represent reinforced concrete structures. Comparisons of the cumulative demand between the three distinct numerical models showed that the degrading model presented 500\% more demand than the other models. This result suggests that reinforced concrete structures would undergo more cumulative demand than other structures. Thus, deficient RC bridge substructures that are susceptible to crustal earthquakes would be even more susceptible to subduction zone demands.

Quasi-static loading protocols were developed to represent the increase of cumulative inelastic demands in reinforced concrete structure. The inelastic time history results from the degrading numerical model in conjunction with the simplified rainflow counting procedure were used for the development of the protocols. The proposed loading protocols include a larger number of small amplitude inelastic cycles as compared to conventional protocols, revealing that conventional loading protocols commonly used in experimental testing tend to impose unrepresentative drift demands through imposing numerous large inelastic reversals on the component. Despite the higher number of large inelastic cycles, the overall normalized cumulative plastic displacement demands were similar when compared to the proposed protocols.

A representative pre-1970 lightly reinforced and lap-spliced bridge column was studied to observe the effect of the proposed protocols on the behavior of reinforced concrete bridge columns. Experimental results of deficient square RC columns showed that the proposed subduction protocols influenced the response of reinforced concrete columns due to an increase in the overall number of inelastic cycles. This influence in response was observed in a reduced displacement capacity and strength resistance of the column subjected to one of the subduction protocols as compared to a conventional protocol. The assessment of $\mathrm{RC}$ bridge columns through representative testing load protocols would play a key role in the future establishment of limit 
states, failure modes and acceptance criteria to be applied in the design of bridge columns and should be considered when megathrust subduction earthquake hazard affects the design criteria.

The design implementation of buckling restrained braces as a transverse direction retrofit measure for reinforced concrete multi-column bridge bents was presented through 4 main steps, which are assessment of the as-built bent, BRB design, design of connections and capacity check. The limit states for the connection were also outlined. The design concept aims to concentrate all the induced energy during an earthquake event in disposable elements, while the reinforced concrete bent behavior is essentially in the elastic range. Based on this retrofit strategy, a case study of a representative bridge found in Oregon was discussed. The numerical results showed that by implementing buckling restrained braces, the retrofitted bent was significantly stiffer than conventionally retrofitted and yet provided for ductile response without significant damage to the concrete elements and could be a suitable retrofit measure for successfully achieving performance based dual-level design approaches. Even though, the proposed design implementation was developed for a diagonal configuration in mind, its application can be extended to other cases.

The experimental results of seismic performances of seismically deficient bridge bents retrofitted using buckling restrained braces in a diagonal configuration. Retrofitted and unretrofitted cases were tested using cyclic loading protocols representative of the displacement demands in RC bridge bents subjected to subduction zone earthquakes. The retrofitted RC bridge bent was designed to perform elastically or with minor inelastic excursions within the original bent throughout the different seismic hazard design levels. Two BRB designs were considered in the study in an effort to assess the influence of BRB stiffness on the overall structural performance. A novel gusset plate to $\mathrm{RC}$ bent connection was used, in which the gusset plates were directly connected to the horizontal RC elements without interfering with the columns. The results of these large-scale experiments successfully demonstrated the effectiveness of utilizing buckling restrained braces for achieving high displacement ductility of the retrofitted structure, while also controlling the damage of the existing vulnerable reinforced concrete bent up to the design performance levels. Further, the experimental results showed that the BRB retrofit measure can achieve operational performance levels under both design levels. No damage was observed in the connection regions of the brace throughout the loading history, leaving the potential for replaceability of the sacrificial BRB element. The potential for improving the overall seismic behavior and the design performance levels with BRBs offers bridge design professionals a viable method for performance driven retrofit of multi-column reinforced concrete bridge bents.

The experimental results also indicated that despite the detailing deficiencies of the square $\mathrm{RC}$ bridge column and the multi-column RC bridge bent built before 1970 in the Pacific Northwest, the cyclic response of the columns and the unretrofitted bent exhibited moderately ductile performance. The moderately ductile performance is likely a result of a relatively long lap splice length and low axial column loads.

Fragility curves that were developed by using nonlinear static analysis showed that the representative as-built bridge bent is more susceptible to subduction zone ground motions as compared to the response of the $\mathrm{RC}$ bent retrofitted with buckling restrained braces. As a result, the analyses of the fragility curves revealed the effectiveness of the proposed retrofit measure in mitigating probable damage undergo by the structure during a major seismic event. The fragility 
curves and the retrofit measure as presented in this study aim to improve the criteria for retrofitting prioritization, estimation of potential losses and help with the decision of selecting a suitable retrofit measure in areas prone to subduction zone mega earthquakes.

\section{$9.2 \quad$ FUTURE WORK}

The implementation and experimental validation of buckling restrained braces as retrofit measure for $\mathrm{RC}$ bridges was the primary outcome of this study. Future research on the implementation of buckling restrained braces as seismic retrofit of multi-column bridge bent and on the field of retrofit measures considering subduction zone earthquakes would be very beneficial and is summarized below.

- Quasi-static loading protocols were utilized in the experimental part of this project. Dynamic or Pseudo-dynamic tests should be conducted to further validate the proposed loading protocols and the proposed retrofit measure.

- More case studies with different configurations should be analyzed in order to broad the applicability of the BRB system, and to assess the effect of the retrofit measure by using different BRB configurations.

- Impact of the implementation of BRB on landscape, issues with debris collection and long term effects, such as corrosion, should be investigated.

- Different connections between gusset plate and concrete should be analytically and experimentally studied to ensure satisfactory seismic performance.

- Comparisons between the retrofit measure presented in this report and others currently available in the market should be performed. Cost effectiveness should be included in such analysis.

- Experimental studies are needed to validate other structural fuses such as the eccentrically braced frame system that was briefly discussed in this report.

- The models in this study assumed rigid footings and pile-footing interaction was not considered. Soil and liquefaction effects should be incorporated into the models.

- Fragility curves can be improved by further studying the use of different bridge bent models with variable geometry and material properties, and also by performing incremental dynamic analysis. 


\subsection{REFERENCES}

AASHTO. Guide Specifications for LRFD Seismic Bridge Design. American Association of State Highway and Transportation Officials. 2009.

ACI 318-11. Building Code Requirements for Structural Concrete and Commentary. American Concrete Institute. 2011.

ACI 374.2R-13. Guide for Testing Reinforced Concrete Structural Elements under Slowly Applied Simulated Seismic Loads. American Concrete Institute. Farmington Hills, MI, 2013.

AISC. Seismic Provisions for Structural Steel Buildings. American Institute of Steel Construction. Chicago, IL, 2010.

AISC. Steel Construction Manual. American Institute of Steel Construction. 2011.

Andrawes, B., M. Shin, and N. Wierschem. Active Confinement of Reinforced Concrete Bridge Columns Using Shape Memory Alloys. Journal of Bridge Engineering, Vol. 15, No. 1, 2010, pp. 81-89.

ASCE/SEI 41-06. ASCE/SEI 41-06: Seismic Rehabilitation of Existing Buildings. American Society of Civil Engineers. Reston, VA, 2007.

ASTM E1049-85. Standard Practices for Cycle Counting in Fatigue Analysis. West Conshohocken, PA, 2005.

ATC. Seismic Vulnerability and Impact of Disruption of Lifelines in the Conterminous United States. ATC-25, ATC. Redwood City, CA, 1991.

ATC-24. Guidelines for Cyclic Seismic Testing of Components of Steel Structures for Buildings. Applied Technology Council. Redwood City, CA, 1992.

Atkinson, G.M., and D.M. Boore. Empirical Ground-Motion Relations for Subduction Zone Earthquakes and Their Application to Cascadia and Other Regions. Bulletin of the Seismological Society of America, Vol. 93, No.4, 2003, pp. 1703-1729.

Atwater, B.F., S. Musumi-Rokkaku, K. Satake, Y. Tsuji, K. Ueda, and D. Yamaguchi. 2005. The Orphan Tsunami of 1700: Japanese Clues to a Parent Earthquake in North America. US Geological Survey Professional Pater, No. 1707, 2005, pp. 3-123.

Badoux, M., and J.O., Jirsa. Steel Bracing of RC Frames for Seismic Retrofitting. Journal of Structural Engineering, Vol. 116, No. 1, 1990, pp. 55-74. 
Bai, Y., A. Kawano, S. Matsuo, and K. Odowara. Seismic Responses of High-Rise Buildings Subjected to Extreme Ground Motions of Tohoku Earthquakes in Japan. Advanced Materials Research, Vol. 374-377, 2012, pp. 2118-2125.

Basoz, N., and A. Kiremidjian. Evaluation of Bridge Damage Data from the Loma Prieta and Northridge, CA Earthquakes. Report No. MCEER-98-0004., Multidisciplinary Center for Earthquake Engineering Research. Buffalo, NY, 1998.

Biskinis, D., and M. Fardis. Deformations of Concrete Members at Yielding and Ultimate under Monotonic or Cyclic Loading (Including Repaired and Retrofitted Members). Report No. SEE 2009-0, Department of Civil Engineering, University of Patras. Patras, Greece, 2009.

Bolt, B. 1969. Duration of Strong Motion. Proceedings of the 4th World Conference on Earthquake Engineering. Santiago, Chile, 1969, pp. 1304-1315.

Borg, R., T. Rossetto, and H. Varum. The Effect of the Number of Response Cycles on the Behaviour of Reinforced Concrete Elements Subject to Cyclic Loading. 15th World Conference on Earthquake Engineering. Lisboa, 2012.

Bouadi, A. Use of Eccentrically Braced Frames (EBFs) for Seismic Strengthening of Reinforced Concrete Frame. PhD Thesis, The University of Texas at Austin. 1994.

Bracci, J. M., A. M. Reinhorn, and J. B. \& Mander. Seismic Retrofit of Reinforced Concrete Buildings Designed for Gravity Loads: Performance of Structural Model. ACI Structural Journal, Vol. 92, No. 6, 1995.

Caltrans. Earthquake Retrofit Guidelines for Bridges. Memo to Designers 20-1, California Department of Transportation. Sacramento, CA, 1996.

Caltrans. Seismic Design Criteria. California Department of Transportation. Sacramento, CA, 2013.

Chai, Y.H., M.J.N Priestley, and F. Seible. Retrofit of Bridge Columns for Enhanced Seismic Performance. Seismic Assessment and Retrofit of Bridges, 1991, pp. 177-196.

Chai, Y.H., M.J.N. Priestley, and F. Seible. Flexural Retrofit of Circular Reinforced Concrete Bridge Columns by Steel Jackets. Report Number SSRP-91/06, University of California, Department of Applied Mechanics and Engineering Sciences. San Diego, CA, 1991.

Chai, Y.H., M.J.N. Priestley, and F. Seible. Retrofit of Bridge Columns for Enhanced Seismic Performance. Report Number SSRP-91/03, University of California, Department of Applied Mechanics and Engineering Sciences. San Diego, CA, 1992.

Chandramohan, R., T. Lin, J. Baker, and G. Deierlein. Influence of Ground Motion Spectral Shape and Duration on Seismic Collapse Risk. 10CUEE CONFERENCE PROCEEDINGS, 10th International Conference on Urban Earthquake Engineering. Tokyo, Japan, 2013. 
Cheung, P., T. Pauley, and R. Park. New Zealand Tests on Full-Scale Reinforced Concrete Beam-Column-Slab Sub-assemblages Designed for Earthquake Resistance. ACI Special Publication SP 123-1, American Concrete Institute. Detroit, MI, 1991.

Clark, P., I. Aiken, K. Kasai, E. Ko, and I. Kimura. Design Procedures for Buildings Incorporating Hysteretic Damping Devices. In Proceedings 68th annual convention. SEAOC. Santa Barbara, CA, 1999, pp. 355-371.

Clough, R., and S. Jonhnston. Effect of Stiffness Degradation on Earthquake Ductility Requirements. Proceedings of Japan Earthquake Engineering Symposium. Tokyo, Japan, 1966.

Computers and Structures Inc., CSI. 2011. SAP2000 Integrated Software for Structural Analysis and Design. Version 15.1.0. Berkeley, CA, 2011.

Connor, J.J., A. Wada, M. Iwata, and Y.H. Huang. Damage-Controlled Structures. I: Preliminary Design Methodology for Seimically Active Regions. Journal of Structural Engineering, Vol. 123, No. 4, 1997, pp. 423-431.

Cosenza, C., G. Manfredi, and R. Ramasco. The Use of Damage Functionals in Earthquake Engineering: a Comparison Between Different Methods. Earthquake Engineering \& Structural Dynamics, Vol. 22, No. 10, 1993, pp. 855-868.

COSMOS. Consortium of Organizations for Strong Motion Observation Systems. 2012. http://www.cosmos-eq.org/. Accessed 2015.

DesRoches, R., B. Nielson, and E. Choi. Seismic Fragility of Typical Bridges in Moderate Seismic Zones. Engineering Structures, 2003, pp. 187-199.

Dobry, R., I. Idriss, and E. Ng. Duration Characteristics of Horizontal Components of Strong Motion Earthquake Records. Bulletin of the Seismological Society of America, Vol. 68, No. 5, 1978, pp. 1487-1520.

Dyngeland, T. Retroffiting of Bridges and Building Structures - A Literature Survey. Special Publication No. I.98.33, Ispra, 19p, European Laboratory for Structural Assessment, JRC-ISIS. 1998.

El-Bahey, S., and M. Bruneau. Buckling Restrained Braces as Structural Fuses for the Seismic Retrofit of Reinforced Concrete Bridge Bents. Engineering Structures, Vol. 33, 2011, pp. 10521061 .

ElGawady, M., M. Endeshaw, D. McLean, and R. Sack. Retrofitting of Rectangular Columns with Deficient Lap Splices. Journal of Composites for Construction, Vol. 14, No. 1, 2010, pp. 22-35. 
FEMA 356. 2000. Prestandard and commentary for the seismic rehabilitation of buildings. Washington, DC: Report No. FEMA 356. Prepared by American Society of Civil Engineers. Prepared for Federal Emergency Management Agency.

FEMA 440A. Effects of Strength and Stiffness Degradation on Seismic Response. Federal Emergency Management Agency. Washington D.C., 2009.

FEMA 461. Interim Protocols for Determining Seismic Performance Characteristics of Structural and Nonstructural Components Through Laboratory Testing. FEMA 461, Federal Emergency Managment Agency. 2007.

FEMA P695. Quantification of Building Seismic Performance Factors. Federal Emergency Managment Agency. Washington, D.C., 2009.

FHWA. Seismic Retrofitting Manual for Highway Structures: Part 1-Bridges. Publication No. FHWA-HRT-06-032. Department of Transportation, Federal Highway Administration. McLean, VA, 2006.

Ghobarah, A., and H.A. Elfath. Rehabilitation of a Reinforced Concrete Frame Using Eccentric Steel Bracing. Engineering Structures, Vol. 23, No. 7, 2001, pp. 745-755.

Ghobarah, A., H. Abou-Elfath, and A. Biddah. Response-Based Damage Assessment of Structures. Earthquake Engineering and Sructural Dynamics, Vol. 28, No. 1, 1999, pp. 79-104.

Goda, K., and G. Atkinson. Seismic Demand Estimation of Inelastic SDOF Systems for Earthquakes in Japan. Bulletin of the Seismological Society of America, Vol. 99, No. 6, 2009, pp. 3285-3299.

Goldfinger, C., K. Grijalva, R. Burgmann, A.E. Morey, J.E. Johnson, C.H. Nelson, J. GutierrezPastor, A. Ericsson, E. Karabanov, J.D. Chaytor, J. Patton, and E. Gracia. Late Holocene Rupture of the Northern SAn Andreas Fault and Possible Stress Linkage to the Cascadia Subduction Zone. Bulletin of the Seismological Society of America, Vol. 98, No. 2, 2008, pp. 861-889.

Gregor, N., W. Silva, I. Wong, and R. Youngs. Ground-Motion Attenuation Relationships for Cascadia Subduction Zone Megathrust Earthquakes Based on a Stochastic Finite-Fault Model. Bulletin of the Seismological Society of America, Vol. 92, No. 5. 2002, pp. 1923-1932.

Grossi, P. Earthquake Damage Assessment From Expert Opinion to Fragility Curves. 8th ASCE Specialty Conference on Probabilistic Mechanics and Structural Reliability. Notre Dame, IN, 2000 .

Gulkan, P, and M.A. Sozen. Inelastic Responses of Reinforced Concrete Structures to Earthquake Motions. Journal of the American Concrete Institute, Vol. 71, No. 12, 1974, pp. 604610. 
Haroun, M.A., and H.M. Elsanadedy. Fiber-Reinforced Plastic Jackets for Ductility Enhancement of Reinforced Concrete Bridge Columns with Poor Lap-Splice Detailing." Journal of Bridge Engineering (ASCE), Vol. 10, No. 6, 2005, pp. 749-757.

http://ascelibrary.org/beo/resource/1/jbenf2/v10/i6/p749 s1?view=fulltext. Accessed May 4, 2012.

Haselton, C., A. Liel, S. Taylor, and G. Deierlein. Beam-Column Element Model Calibrated for Predicting Flexural Response Leading to Global Collapse of RC Frame Buildings. PEER Report 2007/03, Pacific Earthquake Engineering Research Center. Berkeley, CA, 2008.

Hayashi, T., H. Niwa, and M. Fukuhara. Strengthening Methods of the Existing Reinforced Concrete Buildings. Proceedings of the Seventh World Conference on Earthquake Engineering. 1980, pp. 89-97.

Heaton, T., and S. Hartzell. Source Characteristics of Hypothetical Subduction Earthquakes in the Northwestern United States. Bulletin of the Seismological Society of America, Vol. 76, No. 3, 1986, pp. 675-708.

Heaton, T.H., and H. Kanamori. Seismic Potential Associated with Subduction in the Northwestern United States. Bulletin of the Seismological Society of America, Vol. 74, No. 3, 1984, pp. 933-941.

Hjelmstad, K.D, and E.P. Popov. Seismic Behavior of Active Beam Links in Eccentrically Braced Frames. Report No. UCB/EERC-83/24, Earthquake Engineering Research Center, University of California. Berkeley, CA, 1983.

Hose, Y., and F. Seible. Performance Evaluation Database for Concrete Bridge Components and Systems under Simulated Seismic Loads. PEER Report 1999/11, Pacific Earthquake Engineering Research Center. Berkeley, CA, 1999.

Hoshikuma, J., and Z. Guangfeng. Performance of Seismic Retrofitted Highway Bridges Based on Observation of Damage due to the 2011 Great East Japan Earthquake. Journal of JSCE, Vol. 1, 2013, pp. 343-352.

Hutchinson, T., and R. Wood. Cyclic Load Protocol for Anchored Nonstructural Components and Systems. Earthquake Spectra, Vol. 29, No. 3, 2013, pp. 817-842.

Hwang, H, J.B. Jernigan, and Y. Lin. Expected Seismic Damage to Memphis Highway Systems. 5th U.S. Conference on Lifeline Earthquake Engineering. American Association of Civil Engineers. Seattle, WA, 1999, pp. 1-10.

Hwang, H., J.B. Jernigan, and Y. Lin. Evaluation of Seismic Damage to Memphis Bridges and Highway Systems. Journal of Bridge Engineering, Vol. 5, No. 4, 2000, pp. 322-330.

Hwang, H., J.B. Liu, and Y-H. Chiu. Seismic Fragility Analysis of Highway Bridges. Technical Report, Center for Earthquake Research and Information. Memphis, TN, 2001. 
Ibarra, L., and H. Krawinkler. Global Collapse of Frame Structures under Seismic Excitations (PEER 2005/06). Pacific Earthquake Engineering Research Center, Berkeley, CA, 2005b.

Ibarra, L., R. Medina, and H. Krawinkler. Hysteretic Models that Incorporate Strength and Stiffness Deterioration. Earthquake Engineering and Structural Dynamics, Vol. 34, 2005a, pp. 1489-1511.

Imbsen, R.A. Recommended LRFD Guidelines for the Seismic Design of Highway Bridges. National Cooperative Highway Research Program, No. 20-07/Task 193, Sacramento, CA, 2006.

Itani, A. Cyclic Behavior of Shear Links in Retrofitted Richmond-San Rafael Bridge Towers. Third US-Japan Workshop on Seismic Retrofit of Bridges. Tsubuka Science City, Japan. 1996, pp. 261-268.

Kawashima, K., K. Kosa, Y. Takashi, M. Akiyama, T. Watanabe, G. Nishioka, H. Koga, and H. Matsuzaki. Damages of Bridges during 2011 Great East Japan Earthquake. Proceedings of 43rd Joint Meeting, US-Japan Panel on Wind and Seismic Effects. UJNR, Tsukuba Science City, Japan, 2011.

K-Net. Kyoshin Network Database, National Research Institute for Earth Science and Disaster Prevention. (undated). http://www.k-net.bosai.go.jp/. Accessed 2015.

Krawinkler, H. Cyclic Loading Histories for Seismic Experimentation on Structural Components. Earthquake Spectra, Vol. 12, No. 1, 1996, pp. 1-12.

Krawinkler, H., A. Gupta, R. Medina, and N. Luco. Loading Histories for Seismic Performance Testing of SMRF Components and Assemblies. SAC Joint Venture. $2000 \mathrm{~b}$.

Krawinkler, H., and A. Nassar. Strength and Ductility Demands for SDOF and MDOF Systems Subjected to Whittier Narrows Earthquake Ground Motions. CSMIP-1990, California Dept. of Conservation, Sacramento, CA, 1990.

Krawinkler, H., F. Parisi, L. Ibarra, A. Ayoub, and R. Medina. Development of a Testing Protocol for Woodframe Structures. CUREE Publication, Richmond, CA, No. W-02, 2000a.

Krawinkler, H., M. Zohrei, B. Lashkari-Irvani, N. Cofie, and H. Hadidi-Tamjed. Recommendations for Experimental Studies on the Seismic Behavior of Steel Components and Materials. John A. Blumel Center, Department of Civil Engineering, Stanford University, 1983.

Krawinkler, H., R. Medina, and B. Alavi. Seismic Drift and Ductility Demand and Their Dependence on Ground Motions. Engineering Structures, Vol. 25, No. 5, 2003, pp. 637-653.

Kunnath, S., A. El-Bahy, A. Taylor, and W. Stone. Cumulative Seismic Damage of Reinforced Concrete Damage Piers. Building and Fire Research Laboratory, National Institute of Standards and Technology, Gaithersburg, MD, 1997. 
Lanning, J., G. Benzoni, and C. Uang. The Feasibility of Using Buckling-Restrained Braces for Long-Span Bridges. A Case Study. Report No. CA12-2149. State of California. Department of Transportation, La Jolla, CA, 2011.

Liao, W-I., and C-H. Loh. Preliminary Study on the Fragility Curves for Highway Bridges in Taiwan. Journal of the Chinese Institute of Engineers, Vol. 27, No. 3, 2004, pp. 367-375.

Lignos, D., and H. Krawinkler. Sidesway Collapse of Deteriorating Structural Systems Under Seismic Excitations. Report No. 177. The John A. Blume Earthquake Engineering Center, Stanford, CA, 2012.

Luco, N., and P. Bazzurro. Does Amplitude Scaling of Ground Motion Records Result in Biased Nonlinear Structural Drift Responses? Earthquake Engineering and Structural Dynamics, Vol. 36, 1007, pp. 1813-1835.

Mackie, K., and B. Stojadinovic. Fragility Basis for California Highway Overpass Bridge Seismic Decision Making. PEER Report 2005/02, Pacific Earthquake Engineering Research Center. University of California, Berkeley, CA, 2005.

Mander, J., and N. Basöz. Enhancement of the Highway Transportation Lifeline Module in HAZUS. Prepared for National Institute of Building Sciences, NIBS, 1999.

Mander, J.B., M.J.N. Priestley, and R. Park. Theoretical Stress-Strain Model for Confined Concrete. Journal of Structural Engineering (ASCE), Vol. 114, No. 3, 1984, pp. 1804-1826.

Manheim, D.N. On the Design of Eccentrically Braced Frames. PhD Thesis. University of California, Berkeley, CA, 1982.

Mazzolani, F.M. Steel Bracing Systems for the Seismic Upgrading of RC Structures. Steel Construction, 2009, pp. 235-242.

McDaniel, C. Seismic Assessment and Retrofit of Existing Multi-Column Bent Bridges. Research Report No. WA-RD 639.1, Washington State Department of Transportation (WSDOT), Olympia, WA, 2006.

McDaniel, C., W. Cofer, D. McLean, and A. Rodriguez-Merek. Performance of pre-1975 Concrete Bridges in Cascadia Subduction-Zone Earthquakes. FHWA Contract DTFH61-03-C00204. Washington State Transportation Center (TRAC), Pullman, WA, 2006.

McLean, D., S. Kuebler, and T. Mealy. Seismic Performance and Retrofit of Multi-Column Bridge Bents. Technical Report, No. WA-RD 449.1, Pullman, Washington: Washington State Transportation Center (TRAC), 1998.

Medina, R., and H. Krawinkler. Seismic Demands for Nondeteriorating Frame Structures and Their Dependence on Ground Motions. Report No. 133, Department of Civil and Environmental Engineering, Stanford University, 2003. 
Mehary, S., P. Dusicka, and R. Bazaez. Cyclic Behavior of As-built and CFRP Wrap Retrofitted Square Reinforced Concrete Bridge Columns. Network for Earthquake Engineering Simulation (distributor), Paper, 2014, DOI:10.4231/D3B27PR73.

Mehary, S.T., and P. Dusicka. Prioritization for Seismic Retrofit with Statewide Transportation Assessment. Technical Report No SRS 500-480 OTREC-RR-08-01, Oregon Department of Transportation. Portland State University, Portland, OR, 2015.

Midorikawa, S., H. Miura, and H. Si. Preliminary Analysis for Characteristics of Strong Ground Motion from Gigantic Earthquakes. Paper No.0941: Proceedings of 15th World Conference on Earthquake Engineering. 2012.

Muntasir Billah, A.H.M., and M. Shahria Alam. Seismic Fragility Assessment of Highway Bridges: A State-of-the-Art Review. Structure and Infrastructure Engineering, 2014, pp. 1-29.

Muntasir Billah, A.H.M., M.S Alam, and M. Rahman Bhuiyan. Fragility Analysis of Retrofitted Multicolumn Bridge Bent Subjected to Near-Fault and Far-Field Ground Motion. Journal of Bridge Engineering, Vol. 18, No. 10, 2012, pp. 992-1004.

Murukami, M., and J. Penzien. Nonlinear Response Spectra for Probabilistic Seismic Design and Damage Assessment of Reinforced Concrete Structures. Report No. UCS/EERC-75-38, Earthquake Engineering Research Center, University of California, Berkeley, CA, 1975.

Nielson, Bryant G., and Reginald DesRoches. Analytical Seismic Fragility Curves for Typical Bridges in the Central and Southeastern United States. Earthquake Spectra (Earthquake Engineering Research Institute), Vol. 23, No. 3, 2007, pp. 615-633.

ODOT. Bridge Design and Drafting Manual. Section 1 - Design. Oregon: Oregon Department of Transportation. 2014.

OpenSees. Open System for Earthquake Engineering Simulation, Pacific Earthquake Engineering Research Center, University of California, Berkeley, CA. 2011. http://opensees.berkeley.edu. Accessed 2015.

Ou, Y., J. Song, P. Wang, L. Adidharma, K. Chang, and G. Lee. Ground Motion Duration Effects on Hysteretic Behavior of Reinforced Concrete Bridge Columns. Journal of Structural Engineering, Accepted Manuscript. 2013.

Padgett, J.E., and R. DesRoches. Sensitivity of Seismic Response and Fragility to Parameter Uncertainty. Journal of Structural Engineering (ASCE), Vol. 133, No. 12, 2007, pp. 1710-1718.

Padgett, J.E., and R. DesRoches. Retrofitted Bridge Fragility Analysis for Typical Classes of Multispan Bridges. Earthquake Spectra, Vol. 25, No. 1, 2009, pp. 117-141. 
Padgett, J. Retrofitted Bridge Fragility Curves for Assessing the Consequences of an Earthquake Event. Mid-America Earthquake Center. 2005.

http://mae.cee.uiuc.edu/documents/slc online magazine 2005 july padgett.pdf. Accessed

December 11, 2010

Padgett, J.E., and R. DesRoches. 2008. Methodology for the Development of Analytical Fragility Curves for. Earthquake Engineering and Structural Dynamics, Vol. 37, No. 8, 2008, pp. 11571174.

Pan, Y. Seismic Fragility and Risk Management of Highway Bridges in New York State. PHD Thesis. The City University of New York, New York, NY, 2007.

Pantelides, C.P., and J. Gergely. 2008. Seismic Retrofit of Reinforced Concrete Beam-Column T-joints in Bridge Piers with FRP Composite Jackets. ACI Special Publication, Vol. 258, 2008, pp. 1-18.

Paulay, T, and M.J.N Priestley. Seismic Design of Reinforced Concrete and Masonry Structures. John Wiley and Sons, Inc. 1992.

PEER. NGA Database. Pacific Earthquake Engineering Research Center, University of California, Berkeley, CA, 2006. http://peer.berkeley.edu/nga/. Accessed 2015.

PEER/ATC-72-1. Modeling and Acceptance Criteria for Seismic Design and Analysis of Tall Buildings. Prepared by Applied Technology Council. Prepared for Pacific Earthquake Engineering Research Center (PEER), Redwood City, CA, 2010.

Popov, E.P., and M.D. Engelhardt. Seismic Eccentrically Braced Frames. Journal of Constructional Steel Research, Vol. 10, 1988, pp. 321-354.

Priestley, M.J.N., G.M. Calvi, and M.J. Kowalsky. Displacement-Based Seismic Design of Structures. IUSS Press, Italy, 2006.

Priestley, M.J.N., F. Seible, and E. Hines. Seismic Performance of Hollow Rectangular Reinforced Concrete Piers with Highly-Confined Boundary Elements. Report No. SSRP-99/15. Department of Structural Engineering, University of California at San Diego, La Jolla, CA, 2002.

Priestley, M.J.N., F. Seible, and G.M. Calvi. Seismic Design and Retrofit of Bridges. New York: John Wiley and Sons. 1996.

Pujol, S., M. Sozen, and J. Ramirez. Displacement History Effects on Drift Capacity of Reinforced Concrete Columns. ACI Structural Journal, Vol. 103, No. 2, 2006, pp. 253-262.

Pulido, C., M. Saiid Saiidi, D. Sanders, A. Itani, and S. El-Azazy. 2004. Seismic Performance of Two-Column Bents-Part II: Retrofit with Infill Walls. ACI Structural Journal, Vol. 101, No. 5, 2004, pp. 642-649. 
Raghunandan, M., and B. Liel. Effect of Ground Motion Duration on Earthquake-Induced Structural Collapse. Structural Safety, Vol. 41, 2013, pp. 119-133.

Rahnama, M., and H. Krawinkler. Effects of Soft Soils and Hysteresis Model on Seismic Demands. Report No. 108. John A. Blume Earthquake Engineering Center. Department of Civil Engineering, Stanford University. 1993.

Reno, M., and M. Pohll. Seismic Retrofit of California's Auburn-Foresthill Bridge. In Transportation Research Record: Journal of the Transportation Research Board, No. 2201, Transportation Research Board of the National Academies, Washington, D.C., 2010, pp. 83-94.

Richards, P., and C. Uang. Testing Protocol for Short Links in Eccentrically Braced Frames. Journal of Structural Engineering (ASCE), Vol. 132, No. 8, 2006, pp. 1183-1191.

Ridell, R., and N.M. Newmark. Statistical Analysis of the Response of Nonlinear Systems Subjected to Earthquakes. Structural Research Series, No. 468, University of Illinois at UrbanaChampaign. 1979.

Rodriguez, M., and R. Park. Seismic Load Test on Reinforced Concrete Columns Strengthened by Jacketing. ACI Structural Journal, Vol. 91, No. 2, 1994, pp. 150-159.

Ruiz-Garcia, J., and E. Miranda. Inelastic Displacement Ratios for Evaluation of Existing Structures. Earthquake Engineering and Structural Dynamics, Vol. 32, No. 8, 2003, pp. 12371258.

Ruiz-Garcia, J., and E. Miranda. Probabilistic Estimation of Maximum Inelastic Displacement Demands for Performance-Based Design. Earthquake Engineering and Structural Dynamics Vol. 36, No. 9, 2007, pp. 1235-1254.

Saatcioglu, M., and C. Yalcin. External Prestressing Concrete Columns for Improved Seismic Shear Resistance. Journal of Structural Engineering, Vol. 129, No. 8, 2003, pp. 1057-1070.

Seible, F., G.A. Hegemier, M.J.N. Priestley, D. Innamorato, and F. Ho. Carbon Fiber Jacket Retrofit Test of Rectangular Flexural Column with Lap Spliced Reinforcement. Report No. ACTT-95/04, University of California, San Diego: Advanced Composites Technology Transfer Consortium, 1995.

Shinozuka, M., M.Q. Feng, H. Kim, and S. Kim. Nonlinear Static Procedure for Fragility Curve Development. Journal or Engineering Mechanics (ASCE), Vol. 126, No. 12, 2000, pp. 12871295.

Shinozuka, M., M. Q. Feng, J. Lee, and T. Naganuma. Statistical Analysis of Fragility Curves. Journal of Engineering Mechanics (ASCE), Vol. 126, No. 12, 2000, pp. 1224-1231. 
Shinozuka, M., S. Kim, S. Kushiyama, and J. Yi. Fragility Curves of Concrete Bridges Retrofitted By Column Jacketing. Earthquake Engineering and Engineering Vibration, Vol. 1, No. 2, 2002, pp. 195-202.

Shinozuka, M., V. Saxena, G. Deodatis, and M.Q. Feng. Development of Fragility Curves for Multi-Span Reinforced Concrete Bridges. 2001. http://www.civil.columbia.edu/iassar/downloadfiles/SC1 texts/. Accessed September 30, 2010.

Simon, J, J.M. Bracci, and P. Gardoni. Seismic Response and Fragility of Deteriorated Reinforced Concrete Bridges. Journal of Structural Engineering (ASCE), Vol. 136, No. 10, 2010, pp. 1273-1281.

Stapleton, S., C. McDaniel, W. Cofer, and D. McLean. Performance of Lightly Confined Reinforced Concrete Columns in Long-Duration Subduction Zone Earthquakes. Transportation Research Record: Journal of the Transportation Research Board, No. 1928, Transportation Research Board of the National Academies, Washington, D.C., 2005, pp. 185-192.

Stephens, J., and J. Yao. Damage Assessment Using Response Measurements. Journal of Structural Engineering, Vol. 113, No. 4, 1987, pp. 787-801.

Stojadinović, B., and K. Mackie. R-Factor Parameterized Bridge Damage Fragility Curves. Journal of Bridge Engineering (ASCE), Vol. 12, No. 4, 2007, pp. 500-510.

Sugano. Seismic Strengthening of Existing Reinforced Concrete Buildings in Japan. Bulletin of the New Zealand National Society for Earthquake Engineering, Vol. 14, No. 4, 1981, pp. 209222.

Takeda, T., and N. Nielson, and M. Sozen. Reinforced Concrete Response to Simulated Earthquakes. Journal of the Structural Division (ASCE), Vol. 96, No. 12, 1970, pp. 2557-2573.

Takemura, H., and K. Kawashima. Effect of Loading Hysteresis on Ductility Capacity of Reinforced Concrete Bridge Piers. Journal of Structural Engineering, Vol. 43A, 1997, pp. 849858 (in Japanese).

Teng, J.G., J.F. Chen, S.T. Smith, and L. Lam. FRP: Strengthened RC Structures. Wiley, Frontiers in Physics, 2001.

Teng, T-L. Southern California Earthquake Data Center. 1994. http://scedc.caltech.edu/significant/northridge1994.html\#e. Accessed 2015.

Teran, A., and J. Ruiz. Reinforced Concrete Jacketing of Existing Structures. Tenth World Conference in Earthquake Engineering. Balkema, Rotterdam. 1992, pp. 5107-5113.

Thompson, T.J. Effects of Long-Duration Earthquakes on Concrete Bridges with Poorly Confined Columns. M.S. thesis. Washington State University, Pullman, WA. 2004. 
U. Chile. University of Chile, Earthquakes of Chile. 2010.

http://terremotos.ing.uchile.cl/registros/164. Accessed 2015.

USGS, and H.G. Wilshire. USGS page -Oakland. U.S. Geological Survey. 1989.

http://pubs.usgs.gov/dds/dds-29/screens/022sr.jpeg. Accessed 2015.

Vargas, R., and M. Bruneau. Analytical Response and Design of Buildings with Metallic Structural Fuses. I. Journal of Structural Engineering, Vol. 135, No. 4, 2009, pp. 386-393.

Vosooghi, A., and M Saiid Saiidi. Experimental Fragility Curves for Seismic Response of Reinforced Concrete Bridge Columns. ACI Structural Journal, Vol. 109, No. 6, 2012, pp. 825834.

Wen, Y.K. Method for Random Vibration of Hysteretic Systems. Journal of Engineering Mechanics Division (ASCE), Vol. 112, 1976, pp. 249-263.

Wright, T., R. DesRosches, and J. Padgett. Bridge Seismic Retrofitting Practices in the Central and Southeastern United States. Journal of Bridge Engineering, Vol. 16, No. 1, 2011, pp. 82-92.

WSDOT. Bridge Design Manual M 23-50. 2006.

Xiao, Y., H. Wu, and G.R. Martin. Prefabricated Composite Jacketing of RC Columns for Enhanced Shear Strength. Journal of Structural Engineering, Vol. 125, No. 3, 1999, pp. 255264.

Yamamoto, Y., and H. Umemura. Analysis of Reinforced Concrete Frames Retrofitted with Steel Brace. Proceedings of the 10th World Conference on Earthquake Engineering. Balkema, Rotterdam. 1992, pp. 5187-5192.

Yamazaki, F., J. Ohnishi, and S. Tayama. Earthquake Damage Assessment of Expressway Structures in Japan. Asia-Pacific Symposium on Structural Reliability and its Application. Taipei, Taiwan. 1999, pp. 205-214.

Yang, J. Nonlinear Responses of High-Rise Building in Giant Subduction Earthquakes. Ph.D. Thesis. California Institute of Technology, Pasadena, CA. 2009. 
APPENDIX A

EARTHQUAKE CHARACTERISTICS 



\section{APPENDIX A}

\section{EARTHQUAKE CHARACTERISTICS}

The crustal earthquakes from the FEMA 695 far-field record set were chosen as the representative crustal ground motion set in this study. The crustal ground motions along with their general characteristic are summarized in Table A.1

\section{Table A.1: Crustal Ground Motions}

\begin{tabular}{c|c|c|c|c|c|c}
\hline Earthquake & Station & M & $\begin{array}{c}\text { Site } \\
\text { Class }\end{array}$ & $\begin{array}{c}\text { Dist. To } \\
\text { Epicenter (km) }\end{array}$ & $\begin{array}{c}\text { PGA }_{\mathbf{x}} \\
(\mathbf{g})\end{array}$ & $\begin{array}{c}\text { PGA }_{\mathbf{y}} \\
(\mathbf{g})\end{array}$ \\
\hline Loma Prieta & Gilroy Array \#3 & 6.9 & $\mathrm{D}$ & 31 & 0.56 & 0.37 \\
\hline Cape Mendocino & Rio Dell Overpass & 7.0 & $\mathrm{D}$ & 23 & 0.39 & 0.55 \\
\hline Loma Prieta & Capitola & 6.9 & $\mathrm{D}$ & 10 & 0.53 & 0.44 \\
\hline Northridge & Beverly Hills - Mulhol & 6.7 & $\mathrm{D}$ & 13 & 0.42 & - \\
\hline Kobe, Japan & Nishi-Akashi & 6.9 & $\mathrm{C}$ & 9 & 0.51 & 0.50 \\
\hline Chi-Chi, Taiwan & TCU045 & 7.6 & $\mathrm{C}$ & 78 & 0.47 & 0.51 \\
\hline Northridge & Canyon Country-WLC & 6.7 & $\mathrm{D}$ & 27 & 0.41 & 0.48 \\
\hline Superstition Hills & Poe Road (temp) & 6.5 & $\mathrm{D}$ & 11 & 0.45 & 0.30 \\
\hline Landers & Coolwater & 7.3 & $\mathrm{D}$ & 82 & 0.28 & 0.42 \\
\hline Imperial Valley & El Centro Array \#11 & 6.5 & $\mathrm{D}$ & 29 & 0.36 & 0.38 \\
\hline Kocaeli, Turkey & Duzce & 7.5 & $\mathrm{D}$ & 98 & 0.31 & 0.36 \\
\hline Superstition Hills & El Centro Imp. Co. & 6.5 & $\mathrm{D}$ & 36 & 0.36 & 0.26 \\
\hline Imperial Valley & Delta & 6.5 & $\mathrm{D}$ & 34 & 0.24 & 0.35 \\
\hline Friuli, Italy & Tolmezzo & 6.5 & $\mathrm{C}$ & 20 & 0.35 & 0.31 \\
\hline Hector Mine & Hector & 7.1 & $\mathrm{C}$ & 27 & 0.27 & 0.34 \\
\hline Kobe, Japan & Shin-Osaka & 6.9 & $\mathrm{D}$ & 46 & 0.24 & 0.21 \\
\hline Landers & Yermo Fire Station & 7.3 & $\mathrm{D}$ & 86 & 0.24 & 0.15 \\
\hline Kocaeli, Turkey & Arcelik & 7.5 & $\mathrm{C}$ & 54 & 0.22 & 0.15 \\
\hline San Fernando & LA - Hollywood & 6.6 & $\mathrm{D}$ & 40 & 0.21 & 0.17 \\
\hline
\end{tabular}

The subduction zone ground motion sets used in this study were chosen from the 1985

Valparaiso (COSMOS), 2007 Sumatra (COSMOS), 2010 Maule (U. Chile), and 2011 Tohoku (K-Net) earthquakes with distances to the epicenter greater than $100 \mathrm{~km}$ to reduce the number of records and ensure far field response. The subduction ground motions along with their general characteristic are summarized in Table A.2. 
Table A.2: Subduction Ground Motions

\begin{tabular}{|c|c|c|c|c|c|}
\hline Earthquake & Station & Site Class & Dist. To Epicenter (km) & PGA $_{x}(g)$ & $\operatorname{PGA}_{\mathrm{y}}(\mathrm{g})$ \\
\hline \multirow{16}{*}{ 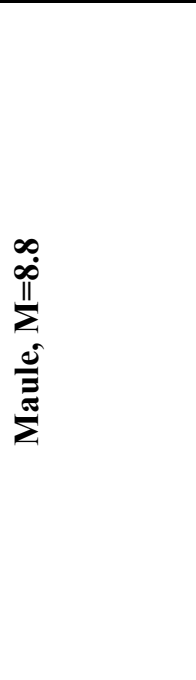 } & Hualane & $\mathrm{C}$ & 195 & 0.69 & 0.43 \\
\hline & Santiago Maipu & $\mathrm{C}$ & 381 & 0.49 & 0.56 \\
\hline & Llolleo & $\mathrm{D}$ & 331 & 0.33 & 0.56 \\
\hline & Curico & $\mathrm{C}$ & 232 & 0.41 & 0.47 \\
\hline & Talca & $\mathrm{C}$ & 348 & 0.47 & 0.42 \\
\hline & Papudo & $\mathrm{C}$ & 404 & 0.30 & 0.42 \\
\hline & Vina del Mar el Salto & $\mathrm{D}$ & 391 & - & 0.35 \\
\hline & Matanzas & $\mathrm{C}$ & 286 & 0.34 & 0.29 \\
\hline & Vina del Mar Centro & $\mathrm{D}$ & 394 & 0.33 & 0.22 \\
\hline & Santiago Centro & $\mathrm{C}$ & 393 & 0.21 & 0.31 \\
\hline & Valparaiso UTFSM & $\mathrm{B}$ & 391 & 0.13 & 0.30 \\
\hline & StgoPenalolen & $\mathrm{C} / \mathrm{D}$ & 393 & 0.29 & 0.30 \\
\hline & StgoPuentealto & $\mathrm{C}$ & 386 & 0.27 & 0.27 \\
\hline & Valparaiso Almendral & $\mathrm{C} / \mathrm{D}$ & 391 & 0.22 & 0.27 \\
\hline & Santiago La Florida & $\mathrm{C}$ & 391 & 0.13 & 0.19 \\
\hline & Valdivia & $\mathrm{C} / \mathrm{D}$ & 394 & 0.13 & 0.09 \\
\hline \multirow{18}{*}{ 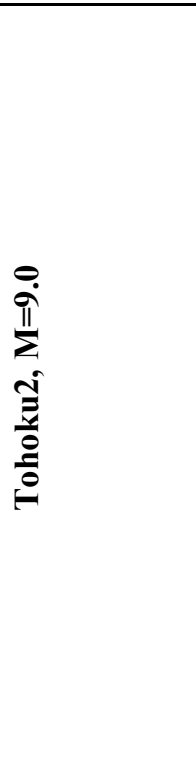 } & Tohwa & $\mathrm{D}$ & 152 & 0.81 & 0.58 \\
\hline & Okhuma & $\mathrm{D}$ & 184 & 0.70 & - \\
\hline & Toyosato & $\mathrm{D}$ & 151 & 0.66 & 0.58 \\
\hline & Kashima & $\mathrm{D}$ & 308 & 0.66 & 0.50 \\
\hline & Nakaminato & $\mathrm{D}$ & 279 & - & 0.56 \\
\hline & Toride & $\mathrm{D}$ & 349 & 0.53 & 0.48 \\
\hline & Mooka & $\mathrm{D}$ & 312 & 0.43 & 0.42 \\
\hline & Aisari & $\mathrm{D}$ & 200 & 0.40 & 0.32 \\
\hline & Iwaki & $\mathrm{D}$ & 206 & 0.30 & 0.38 \\
\hline & Kiryuh & $\mathrm{D}$ & 365 & 0.36 & 0.29 \\
\hline & Kakuda & $E$ & 183 & 0.36 & - \\
\hline & Tsukuba & $\mathrm{D}$ & 330 & - & 0.34 \\
\hline & Fukushima & $\mathrm{D}$ & 213 & 0.30 & 0.33 \\
\hline & Minamidohri & $E$ & 369 & 0.20 & 0.26 \\
\hline & Inage & $\mathrm{D}$ & 369 & 0.21 & 0.24 \\
\hline & Mizue & N/A & 376 & 0.16 & 0.22 \\
\hline & Ohta & $\mathrm{D}$ & 370 & 0.21 & 0.21 \\
\hline & Serinuma & $\mathrm{D}$ & 316 & 0.19 & 0.18 \\
\hline \multirow{16}{*}{ 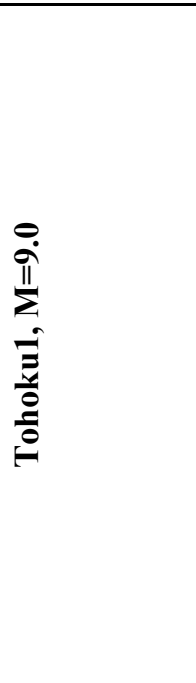 } & Tsukidate & $\mathrm{D}$ & 175 & 1.29 & - \\
\hline & Shiogama & $\mathrm{D}$ & 163 & 2.01 & 0.77 \\
\hline & Hitachi & $\mathrm{D}$ & 258 & 1.21 & - \\
\hline & Sendai & $\mathrm{D}$ & 170 & 1.00 & - \\
\hline & Hokota & $\mathrm{D}$ & 301 & 1.09 & 1.38 \\
\hline & Shirakawa & $\mathrm{D}$ & 259 & 0.97 & 1.32 \\
\hline & Ohmiya & $\mathrm{D}$ & 277 & 1.03 & 1.31 \\
\hline & Motegi & $\mathrm{D}$ & 294 & - & 0.73 \\
\hline & Imaichi & $\mathrm{D}$ & 317 & 1.21 & 1.04 \\
\hline & Hirono & $\mathrm{D}$ & 190 & 0.90 & 1.14 \\
\hline & Kohriyama & $\mathrm{D}$ & 234 & 1.09 & 0.76 \\
\hline & Sakura & $\mathrm{D}$ & 353 & 0.50 & 1.06 \\
\hline & Funehiki & $\mathrm{D}$ & 215 & 0.75 & 1.03 \\
\hline & Ichinoseki & $\mathrm{C}$ & 178 & 0.87 & 1.02 \\
\hline & Kasama & $\mathrm{D}$ & 301 & 0.61 & - \\
\hline & Oshika & $\mathrm{B}$ & 121 & 0.70 & 0.94 \\
\hline
\end{tabular}




\section{APPENDIX B}

SCRIPTING PROCEDURE USED FOR NUMERICAL ANALYSIS 



\section{APPENDIX B}

\section{SCRIPTING PROCEDURE USED FOR NUMERICAL ANALYSIS}

All analysis was conducted using OpenSees and a series of scripts. The process was broken down into several scripts for ease of modification for various SDOF systems, earthquakes, or desired output, and is described below by defining the functions of each script. A schematic view of the process follows in Figure B.1.

- Full Analysis - defines the following parameters and calls on Inelastic Spectra and Cumulative Displacement.

o Ground motions

o Ductility levels

o Degree of damping

o Analysis time step and length

o Period step and range

o SDOF systems

- Inelastic Spectra - calls on Elastic Spectra and Inelastic Analysis, then calculates inelastic spectra.

- Elastic Spectra - uses the SDOF system Elastic Model to produce time history analysis of the desired earthquake(s) at each ductility level and period in the defined range. Maximum values at each period are found to form the spectra.

- Inelastic Analysis - uses results of Elastic Spectra to increase the efficiency of the iterative process (described in the body of the paper) required to reach the desired ductility level, then calls on the SDOF system that was defined in Full Analysis. 
- SDOF Systems - were defined so that the parameters varied with yield force to maintain the integrity of the models at each period and included Bilinear Model, Elastic Nonlinear Model, and Degrading Model. Parameters are more fully defined in Appendix B.

- Cumulative Displacement - calculated the cumulative displacement and residual displacement for at each period of analysis.

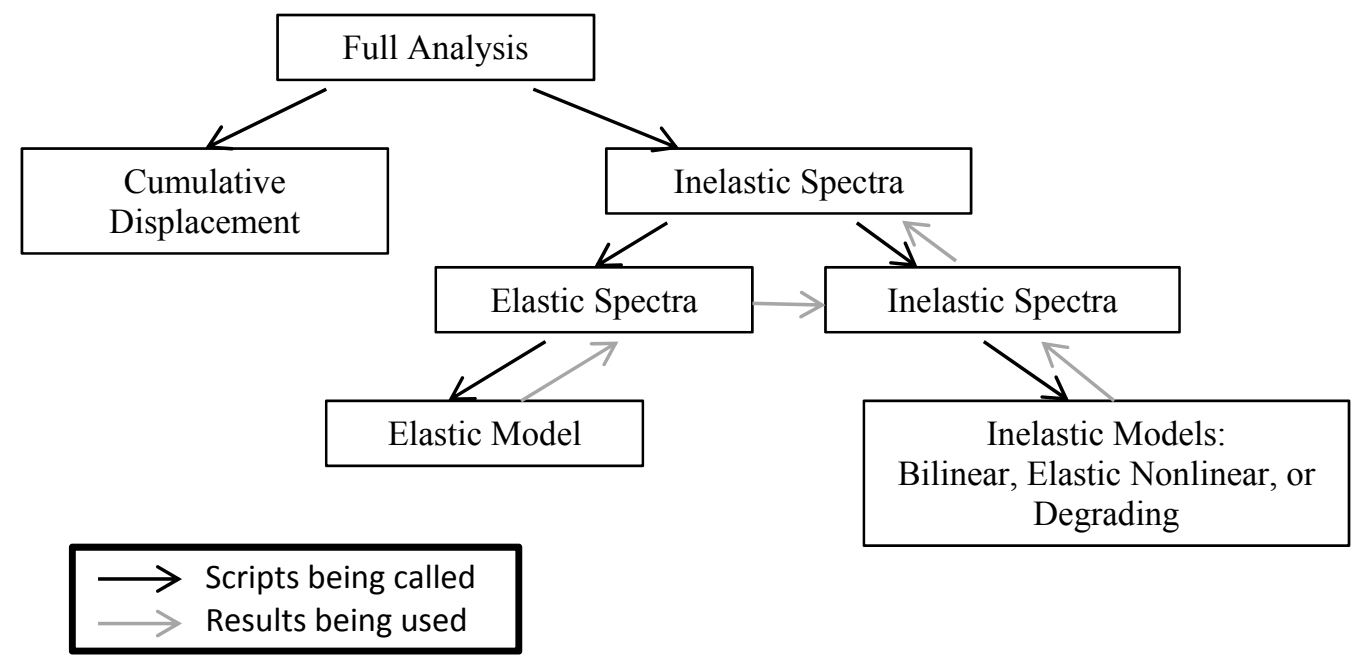

Figure B.1: Scripts used in analysis procedure 


\section{APPENDIX C}

\section{DEVELOPMENT OF THE NUMERICAL MODELS}





\section{APPENDIX C}

\section{DEVELOPMENT OF THE NUMERICAL MODELS}

The hysteretic models were defined as one dimensional SDOF systems using different uniaxial material types. Each material was assigned to a zero length element connecting two nodes, one free and one fixed. In addition to the strength deteriorating material used to describe the degrading model, OpenSees materials Hardening and ElasticBilin were used to describe the bilinear and elastic nonlinear models, respectively. Parameters used to define each of these models are shown in Table C.1.

Table C.1: ElasticBilin and Hardening Material Parameters

\begin{tabular}{c|c|c|c}
\hline \multicolumn{2}{c|}{ ELASTICBILIN } & \multicolumn{2}{c}{ HARDENING } \\
\hline $\begin{array}{c}\text { OpenSees } \\
\text { Variable }\end{array}$ & Defined & $\begin{array}{c}\text { OpenSees } \\
\text { Variable }\end{array}$ & Defined \\
\hline $\mathrm{EP} 1$ & $k=\frac{1}{(T / 2 \pi)^{2}}$ & $\mathrm{E}$ & $k=\frac{1}{(T / 2 \pi)^{2}}$ \\
\hline $\mathrm{EP} 2$ & $0.05 k$ & sigmaY & $f_{v}$ \\
\hline epsP2 & $f_{y} / k$ & H_iso & $0.05 k(1-0.05)$ \\
\hline & & H_kin & 0.0 \\
\hline
\end{tabular}

Analysis of a degrading system in addition to the more simple bilinear and elastic nonlinear systems was deemed necessary to produce results of broader applicability. Several uniaxial materials with degrading properties were available in OpenSees. To determine which was most appropriate for the objectives of this study, a general hysteretic shape was chosen. Then the shape was confirmed and specific parameters calibrated based on experimental data.

Because there are a wide variety of structures and connections which display varying degrees of strength and stiffness deterioration, calibrating the model to experimental data required choosing a hysteretic shape which was at least somewhat structure specific. As this research was a precursor to experimental testing of retrofitted reinforced column bridge piers, a shape was chosen which resembled the type of behavior expected in such structures, but could also be extended to represent similarly behaving structures. FEMA 440A utilized several general hysteretic shapes to study the effects of strength and stiffness degradation in structures. Model 3a, representative of ductile moment frame behavior, was chosen as the shape to most likely characterize the behavior of a retrofitted concrete bridge pier, and the modified Ibarra-MedinaKrawinkler deterioration model with peak-oriented hysteretic response was the OpenSees material with the most similar hysteretic behavior. 
The capacity boundary of the degrading model was defined based on FEMA 440A model 3a. Experimental data was needed to confirm the degree of pinching in the hysteresis, as well as to calibrate the cyclic deterioration parameters. The results of over 400 cyclic tests of reinforced concrete columns were available in the PEER Structural Performance Database. Force displacement data was found for columns of similar dimensions, longitudinal reinforcement ratio, and transverse reinforcement ratio to the experimental column related to this study. Graphing results for several of the columns showed the degree of pinching inherent in the chosen degrading model to be adequate. Experimental results are compared to the degrading model under the same loading protocol as shown in Figure C.1.

Table C.2: Column Properties

\begin{tabular}{c|c|c|c|c}
\hline Column & Type & Span to Depth Ratio & Dimensions (mm) & Axial Load Ratio \\
\hline Lehman 415 & Spiral & 4 & $\mathrm{~d}=609.6$ & 0.072 \\
\hline Henry 415p & Spiral & 4 & $\mathrm{~d}=609.6$ & 0.12 \\
\hline Kowalsky and Moyer 1 & Spiral & 5.33 & $\mathrm{~d}=457.2$ (octagonal) & 0.041 \\
\hline Zahn 7 & Rect & 4 & $400 \mathrm{x} 400$ & 0.223 \\
\hline Column & Long. Reinf. & $\boldsymbol{\rho}_{\mathbf{l}}$ & Transverse Reinf. & $\boldsymbol{\rho}_{\mathbf{t}}$ \\
\hline Lehman 415 & $22-15.9 \mathrm{~mm}$ & 0.0149 & $6.4 \mathrm{~mm} @ 31.8 \mathrm{~mm}$ & 0.007 \\
\hline Henry 415p & $22-15.9 \mathrm{~mm}$ & 0.0149 & $6.4 \mathrm{~mm} @ 31.8 \mathrm{~mm}$ & 0.007 \\
\hline Kowalsky and Moyer 1 & $12-19 \mathrm{~mm}$ & 0.0198 & $9.5 \mathrm{~mm} @ 76.2 \mathrm{~mm}$ & 0.0092 \\
\hline Zahn 7 & $12-16 \mathrm{~mm}$ & 0.0151 & $10 \mathrm{~mm} @ 117 \mathrm{~mm}$ & 0.016
\end{tabular}



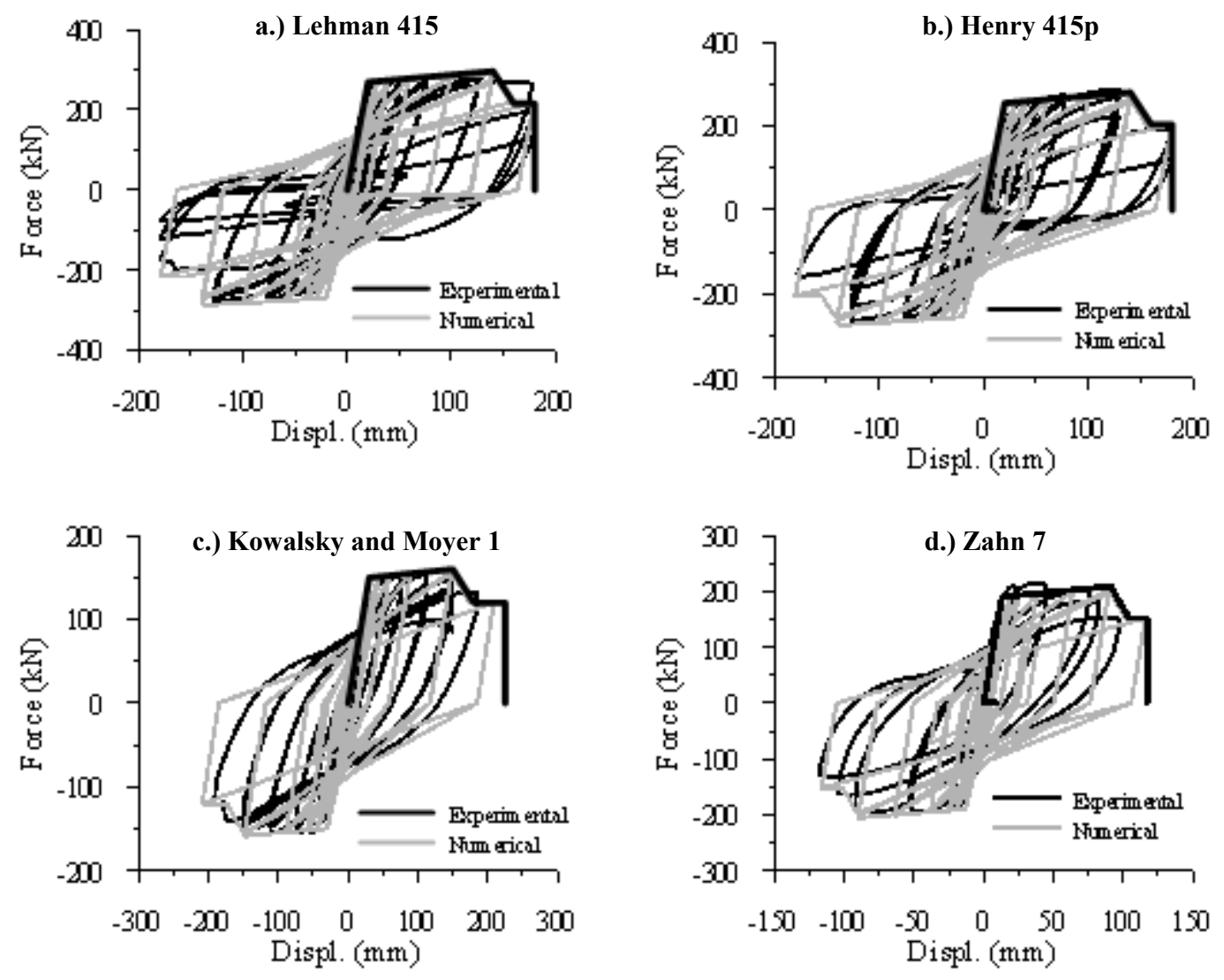

Figure C.1: Experimental column results vs. degrading model

The same experimental results showed the degree of cyclic deterioration to be inconsistent and at least somewhat dependent on the loading protocol which varied. Due to the inconsistencies and because strength deterioration existed in the model as a part of the hysteretic shape, the cyclic deterioration parameters were defined so that no noticeable cyclic deterioration existed. This also aided in simplifying the numerical analysis to a certain degree. Parameters for the degrading model at each ductility level are shown in Table C.3. 
Table C.3: ModIMKPeakOriented Material Parameters

\begin{tabular}{c|c|c|c}
\hline OpenSees Variable & Ductility 2 & Ductility 4 & Ductility 8 \\
\hline K0 & $k=\frac{1}{(T / 2 \pi)^{2}}$ & $k=\frac{1}{(T / 2 \pi)^{2}}$ & $k=\frac{1}{(T / 2 \pi)^{2}}$ \\
\hline As_Plus & 0.0167 & 0.0167 & 0.0167 \\
\hline As_Neg & 0.0167 & 0.0167 & 0.0167 \\
\hline My_Plus & $f_{v}$ & $f_{v}$ & $f_{v}$ \\
\hline My_Neg & $-f_{v}$ & $-f_{v}$ & $200 f_{y}$ \\
\hline Lambda_S & $50 f_{y}$ & $100 f_{y}$ & $200 f_{v}$ \\
\hline Lambda_C & $50 f_{y}$ & $100 f_{v}$ & $200 f_{v}$ \\
\hline Lambda_A & $50 f_{v}$ & $100 f_{v}$ & $200 f_{y}$ \\
\hline Lambda_K & $50 f_{y}$ & $100 f_{y}$ & 1.0 \\
\hline c_S & 1.0 & 1.0 & 1.0 \\
\hline c_C & 1.0 & 1.0 & 1.0 \\
\hline c_A & 1.0 & 1.0 & 1.0 \\
\hline c_K & 1.0 & 1.0 & $5.32\left(f_{y} / k\right)-\left(f_{y} / k\right)$ \\
\hline theta_p_Plus & $1.001\left(f_{y} / k\right)-\left(f_{y} / k\right)$ & $2.44\left(f_{y} / k\right)-\left(f_{y} / k\right)$ & $5.32\left(f_{y} / k\right)-\left(f_{y} / k\right)$ \\
\hline theta_p_Neg & $1.001\left(f_{y} / k\right)-\left(f_{y} / k\right)$ & $2.44\left(f_{y} / k\right)-\left(f_{y} / k\right)$ & $3.56\left(f_{y} / k\right)$ \\
\hline theta_pc_Plus & $2.5\left(f_{y} / k\right)$ & $3.4\left(f_{y} / k\right)$ & $3.56\left(f_{y} / k\right)$ \\
\hline theta_pc_Neg & $2.5\left(f_{y} / k\right)$ & $3.4\left(f_{y} / k\right)$ & 0.8 \\
\hline Res_Pos & 0.8 & 0.8 & 0.8 \\
\hline Res_Neg & 0.8 & 0.8 & $100,000\left(f_{y} / k\right)$ \\
\hline theta_u_Plus & $100,000\left(f_{y} / k\right)$ & $100,000\left(f_{y} / k\right)$ & $100,000\left(f_{y} / k\right)$ \\
\hline theta_u_Neg & $100,000\left(f_{y} / k\right)$ & $100,000\left(f_{y} / k\right)$ & 1.0 \\
\hline D_Plus & 1.0 & 1.0 & 1.0 \\
\hline D_Neg & 1.0 & 1.0 & \\
\hline & & & \\
\hline
\end{tabular}




\section{APPENDIX D}

RESIDUAL DISPLACEMENT UPPER BOUND 



\section{RESIDUAL DISPLACEMENT UPPER BOUND}

Each set of NRD spectra approached an upper bound that was not apparent when analyzing only the mean of each set. For the bilinear without hardening model, the value of the upper bound was the ductility level minus one. The degrading and bilinear with hardening models approached similar upper bounds. The exact upper bound value for each model can be explained by the hysteretic behavior when one assumes each SDOF system will be approximately "centered" at the end of each time history analysis. Figure D.1 along with the equations found in explains the behavior that results in the upper bound value. As an example, the NRD spectra are shown for the Tohoku2 set of the bilinear model in Figure D.2.

a.) Bilinear

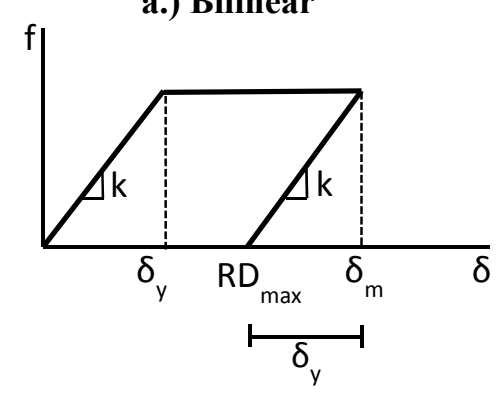

b.) Degrading

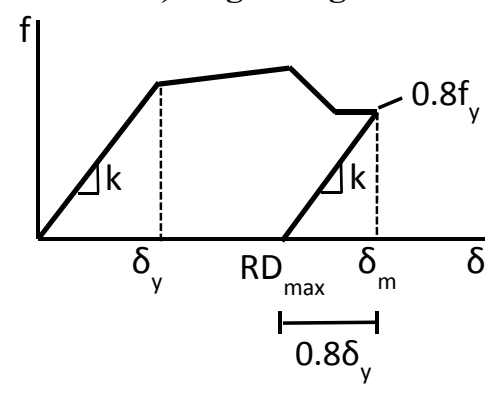

c.) Bilinear with Hardening

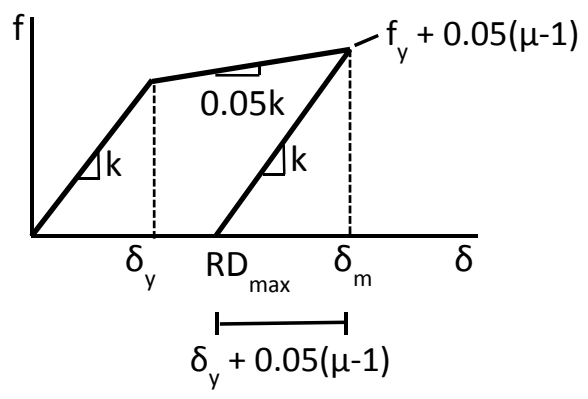

Figure D.1: Hysteretic behavior explaining NRD upper bound 
a.) Ductility 2
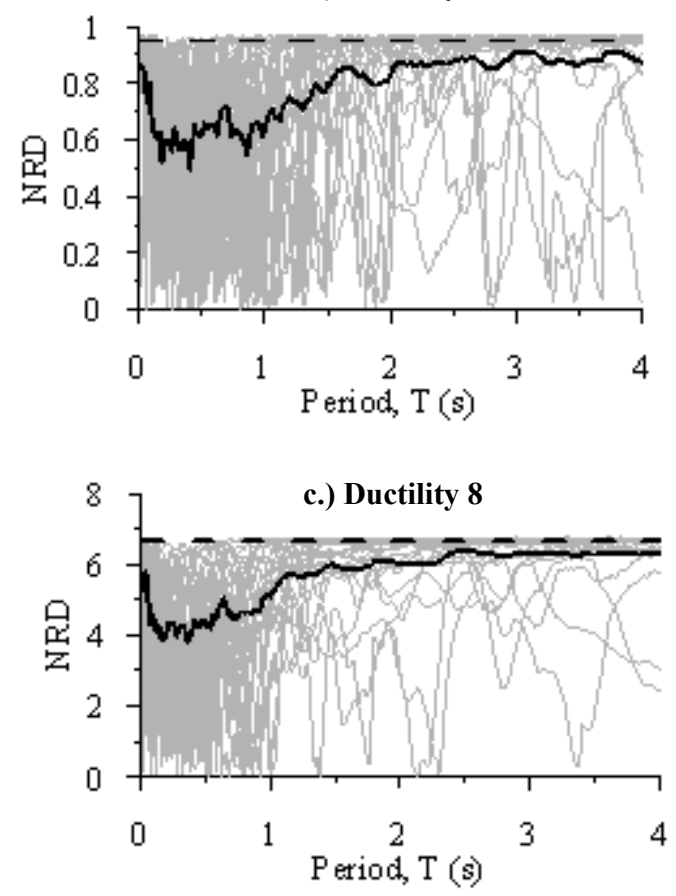

b.) Ductility 4
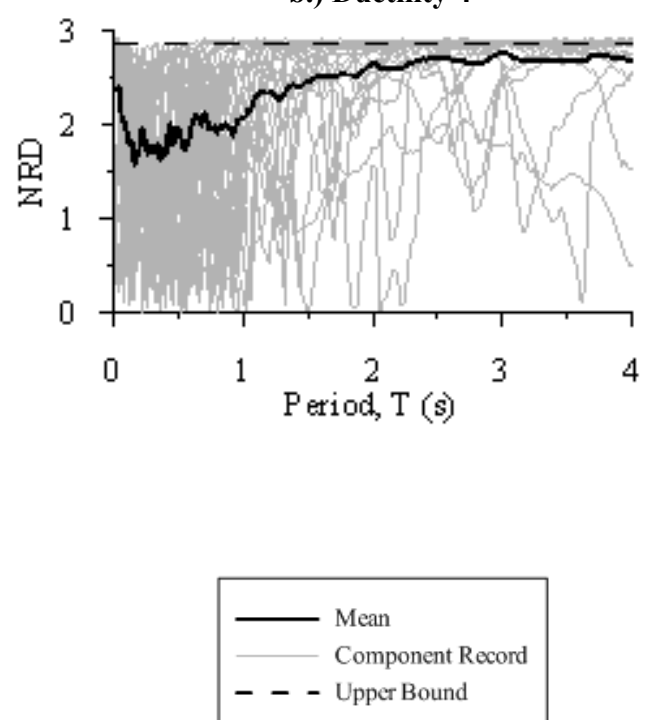

Figure D.2: Tohoku2 Bilinear model NRD

\section{Table D.1: NRD Upper Bound}

\begin{tabular}{c|c|c}
\hline Bilinear Model & Degrading Model & Bilinear with Hardening Model \\
\hline$R D_{\text {max }}=\delta_{m}-\delta_{y}$ & $R D_{\max }=\delta_{m}-0.8 \delta_{y}$ & $R D_{\max }=\delta_{m}-\lambda \delta_{y} *$ \\
$\mu=\frac{\delta_{m}}{\delta_{y}} \rightarrow \delta_{m}=\mu \delta_{y}$ & $\mu=\frac{\delta_{m}}{\delta y} \rightarrow \delta_{m}=\mu \delta_{y}$ & $\mu=\frac{\delta_{m}}{\delta_{y}} \rightarrow \delta_{m}=\mu \delta_{y}$ \\
$\therefore R D_{\max }=\mu \delta_{y}-\delta_{y}$ & $\therefore R D_{\max }=\mu \delta_{y}-0.8 \delta_{y}$ & $\therefore R D_{\max }=\mu \delta_{y}-\lambda \delta_{y}$ \\
$=\delta_{y}(\mu-1)$ & $=\delta_{y}(\mu-0.8)$ & $\delta_{y}(\mu-\lambda)$ \\
$N R D_{\max }=R D_{\max } / \delta_{y}$ & $N R D_{\max }=R D_{\max } / \delta_{y}$ & $N R D_{\max }=R D_{\max } / \delta_{y}$ \\
$\therefore N R D_{\max }=\mu-1$ & $\therefore N R D_{\max }=\mu-0.8$ & $\therefore N R D_{\max }=\mu-\lambda$ \\
\hline
\end{tabular}

$* \lambda$ can be calculated for each ductility value using the above figure.

$\lambda=1.05,1.15,1.35$ for ductility values $2,4,8$, respectively. 


\section{APPENDIX E}

\section{ADDITIONAL RESULTS FROM CHAPTER 2}





\section{ADDITIONAL RESULTS FROM CHAPTER 2}
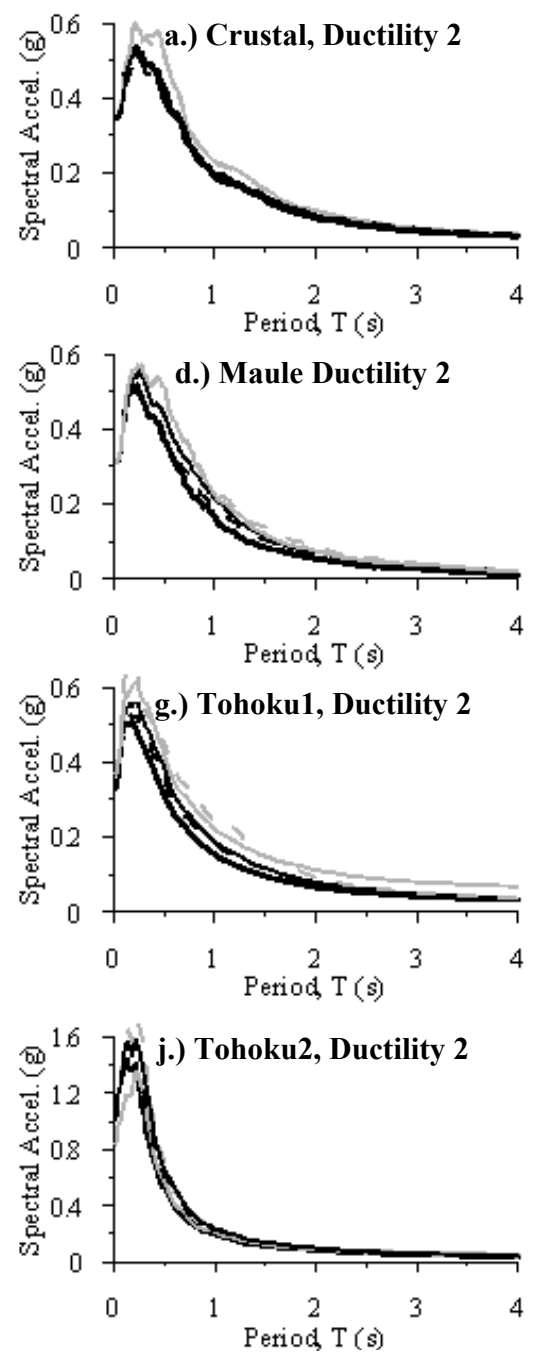
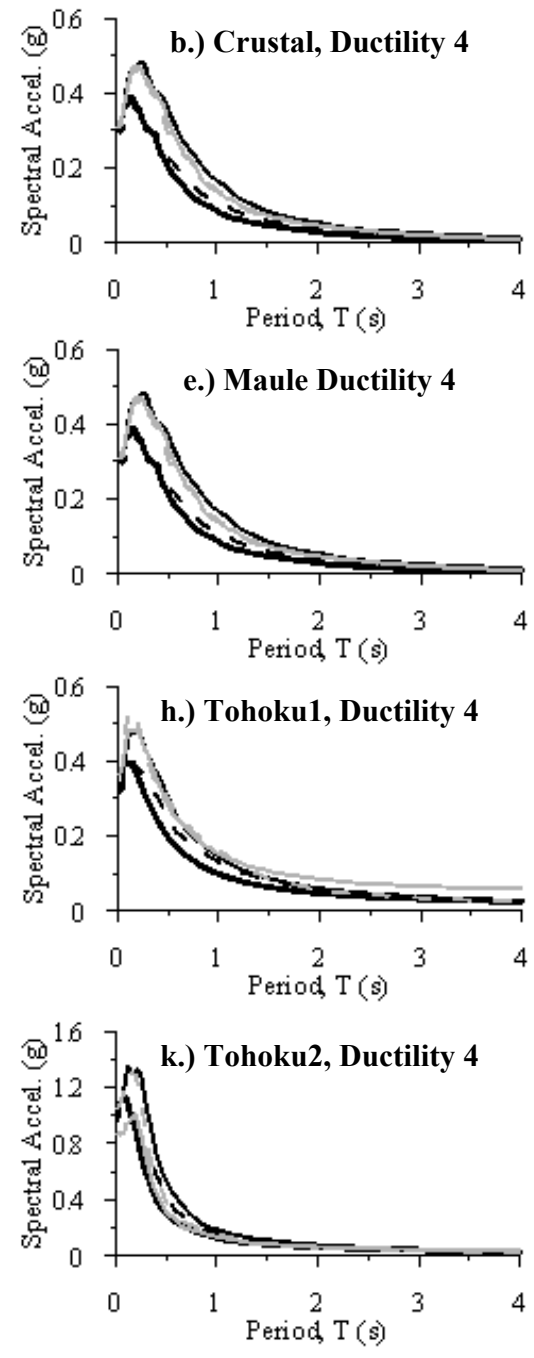
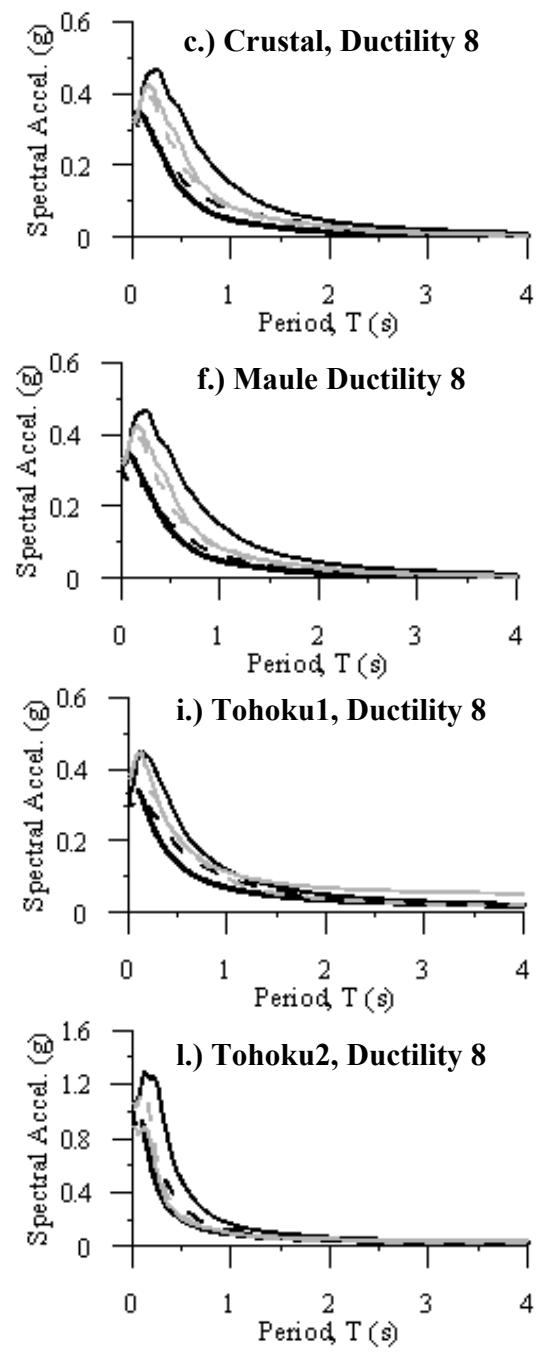

Bilinear $\mathrm{H}$

- BilinearNoH ElasNL H - ElasNL NoH Degrading

Figure E.1: Mean inelastic acceleration spectra by record set 

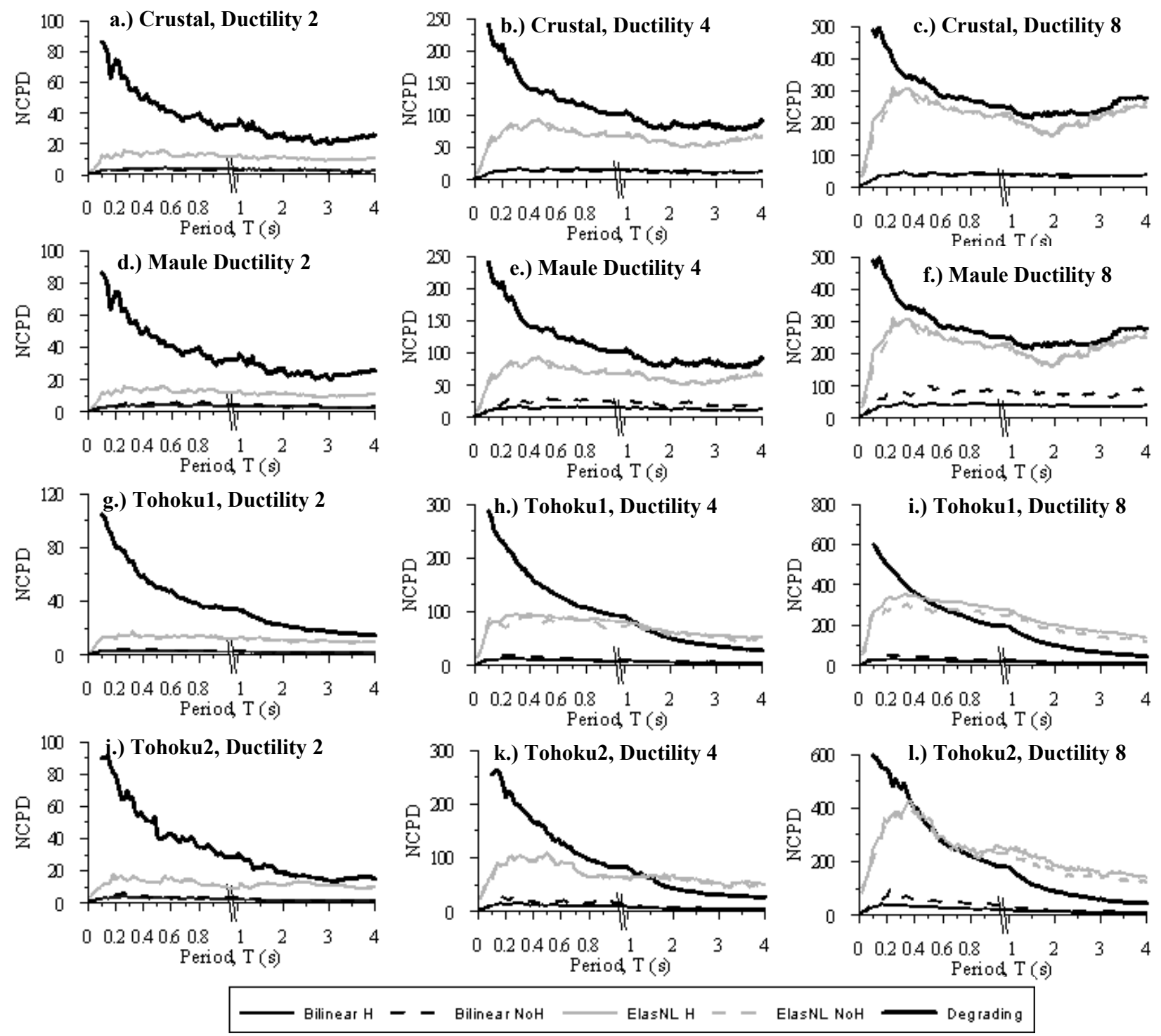

Figure E.2: Mean NCPD spectra by record set 

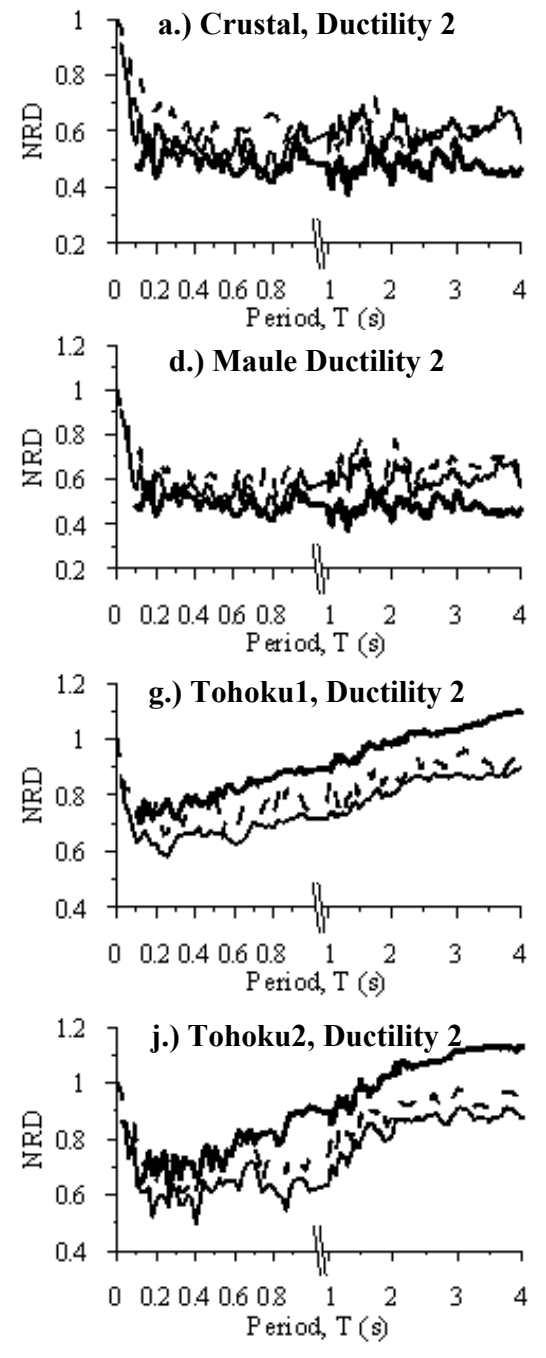
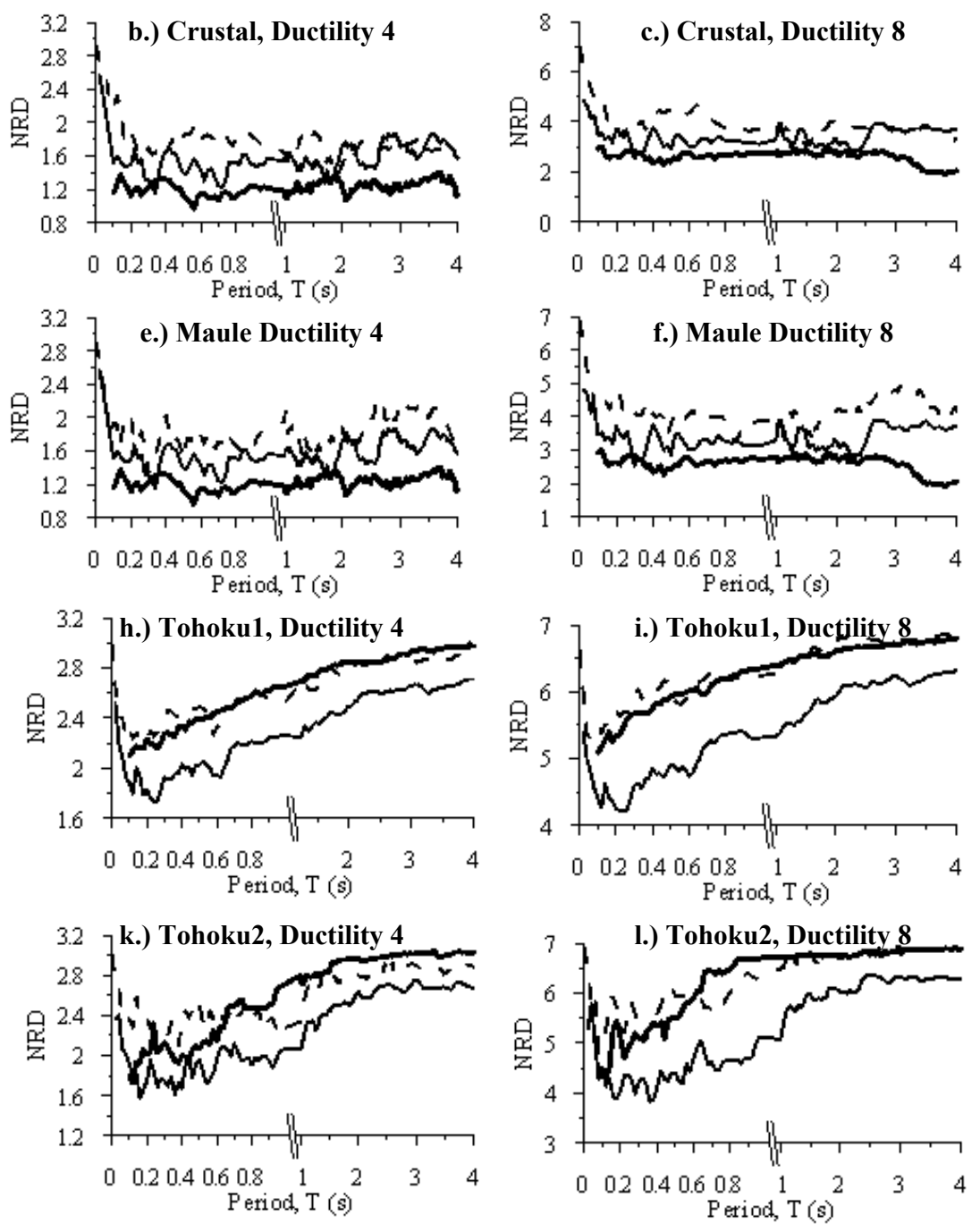

Figure E.3: Mean NRD spectra by record set 
E-1 


\section{APPENDIX F}

\section{CYCLIC LOADING PROTOCOLS CONSIDERING ALL EXCURSIONS}





\section{APPENDIX F}

\section{CYCLIC LOADING PROTOCOLS CONSIDERING ALL EXCURSIONS}

This appendix shows the loading protocols that were developed considering all the inelastic excursions in case experimentalists decide those would be more appropriate for their application. $\mu=2$
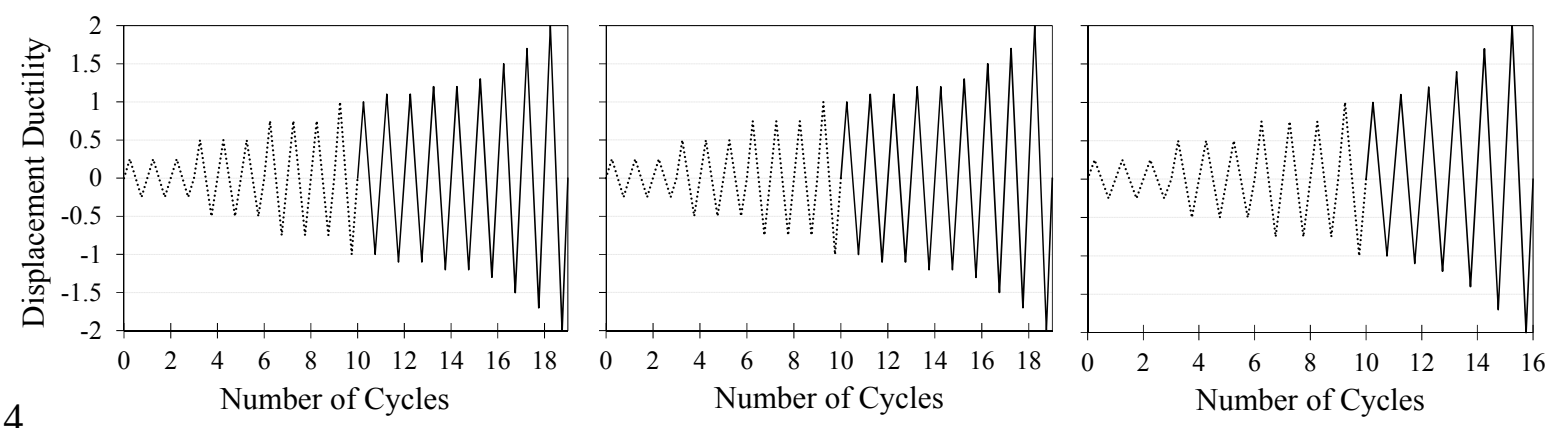
$\mu=4$
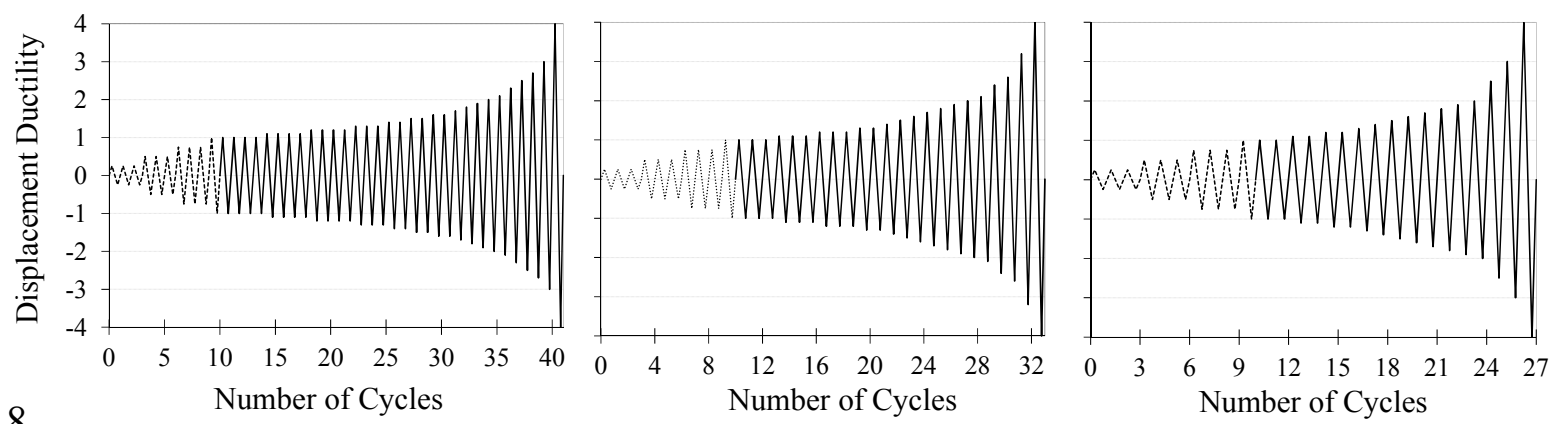

$\mu=8$

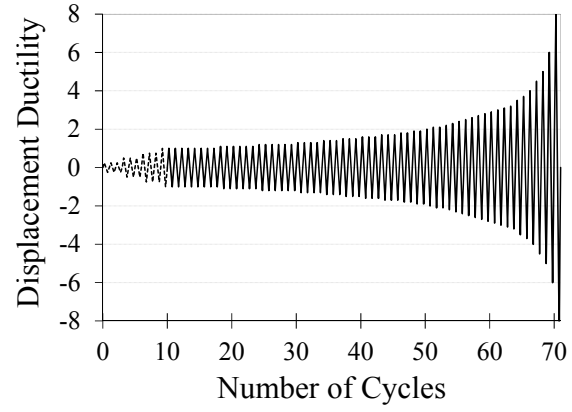

(a)

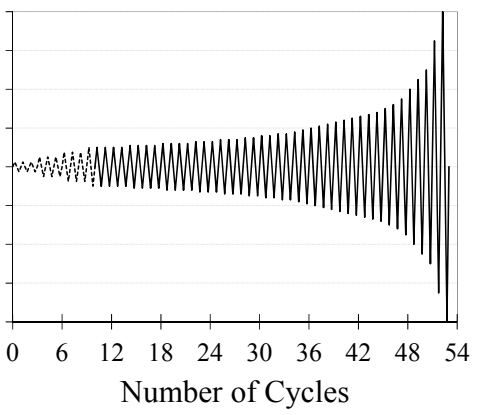

(b)

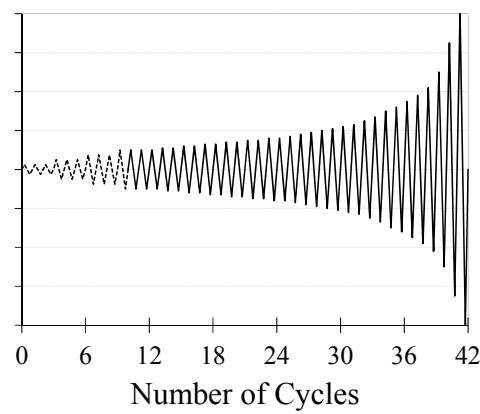

(c)

Figure F.1: Loading protocols considering all excursions for component ductilities $(\mu)=2,4$ and 8. (a) $\mathrm{T}=0.5 \mathrm{sec}$, (b) $\mathrm{T}=1.0 \mathrm{sec}$, (c) $\mathrm{T}=2.0 \mathrm{sec}$. 
Table F.1: Loading protocols utilizing all excursions

\begin{tabular}{|c|c|c|c|c|c|c|}
\hline \multirow{3}{*}{$\begin{array}{c}\text { Cycle } \\
\text { Amplitude } \\
\mathbf{x} \delta_{\text {yield }}\end{array}$} & \multicolumn{6}{|c|}{ Number of Inelastic Cycles } \\
\hline & \multicolumn{3}{|c|}{ Ductility $(\mu=4)$} & \multicolumn{3}{|c|}{ Ductility $(\mu=8)$} \\
\hline & $\mathrm{T}=0.5 \mathrm{~s}$ & $\mathrm{~T}=1.0 \mathrm{~s}$ & $\mathrm{~T}=2.0 \mathrm{~s}$ & $\mathrm{~T}=0.5 \mathrm{~s}$ & $\mathrm{~T}=1.0 \mathrm{~s}$ & $\mathrm{~T}=2.0 \mathrm{~s}$ \\
\hline 1.0 & 4 & 3 & 2 & 8 & 4 & 3 \\
\hline 1.1 & 4 & 3 & 2 & 6 & 4 & 3 \\
\hline 1.2 & 4 & 3 & 2 & 6 & 4 & 2 \\
\hline 1.3 & 3 & 2 & 2 & 4 & 3 & 2 \\
\hline 1.4 & 2 & 1 & 1 & 3 & 3 & 2 \\
\hline 1.5 & 2 & 1 & 1 & 3 & 2 & 1 \\
\hline 1.6 & 2 & 1 & 1 & 3 & 2 & 1 \\
\hline 1.7 & 1 & 1 & - & 3 & 2 & 1 \\
\hline 1.8 & 1 & 1 & 1 & 2 & 2 & 1 \\
\hline 1.9 & 1 & 1 & - & 2 & 1 & 1 \\
\hline 2.0 & 1 & 1 & 1 & 2 & 1 & 1 \\
\hline 2.1 & 1 & - & - & 2 & 1 & 1 \\
\hline 2.2 & - & 1 & - & 1 & 1 & 1 \\
\hline 2.3 & 1 & - & - & 1 & 1 & - \\
\hline 2.4 & - & - & - & 1 & 1 & 1 \\
\hline 2.5 & 1 & 1 & 1 & 1 & 1 & - \\
\hline 2.6 & - & - & - & 1 & 1 & 1 \\
\hline 2.7 & - & - & - & 1 & - & - \\
\hline 2.8 & - & - & - & 1 & 1 & - \\
\hline 2.9 & - & - & - & 1 & - & - \\
\hline 3.0 & 1 & 1 & 1 & 1 & 1 & 1 \\
\hline 3.2 & - & - & - & 1 & 1 & - \\
\hline 3.5 & - & - & - & 1 & 1 & 1 \\
\hline 4.0 & 1 & 1 & 1 & 1 & 1 & 1 \\
\hline 4.5 & & & & 1 & - & 1 \\
\hline 5.0 & & & & 1 & 1 & 1 \\
\hline 5.5 & & & & - & - & - \\
\hline 6.0 & & & & 1 & 1 & 1 \\
\hline 6.5 & & & & - & - & - \\
\hline 7.0 & & & & - & - & 1 \\
\hline 8.0 & & & & 1 & 1 & 1 \\
\hline
\end{tabular}


APPENDIX G

MOMENT-CURVATURE ANALYSIS OF REPRESENTATIVE SQUARE COLUMN 



\section{MOMENT-CURVATURE ANALYSIS OF REPRESENTATIVE SQUARE COLUMN}

\section{$\underline{\text { Column }}$}

Cross Section

Width $\times$ Depth $=24 \times 24$ in

$\mathrm{f}^{\prime}{ }_{\mathrm{c}}=3.5[\mathrm{ksi}], \mathrm{f}^{\prime}{ }_{\mathrm{ce}}=4.5[\mathrm{ksi}], \mathrm{f}_{\mathrm{y}}=60[\mathrm{ksi}], \mathrm{fye}=68[\mathrm{Ksi}]$

Longitudinal Reinforcement: \#10 in each corner

Transverse Reinforcement: \#3@12 [in]

Axial Load: 160 [kip]

\section{Flexural Capacity}

AASHTO specifications (Concrete Modeling): Confined concrete should be computed using Mander's Model. (This formula is based at the first hoop fracture). Maximum strain from Priestley's formula was considered as $\varepsilon_{c u}=0.0109$.

Moment Curvature:

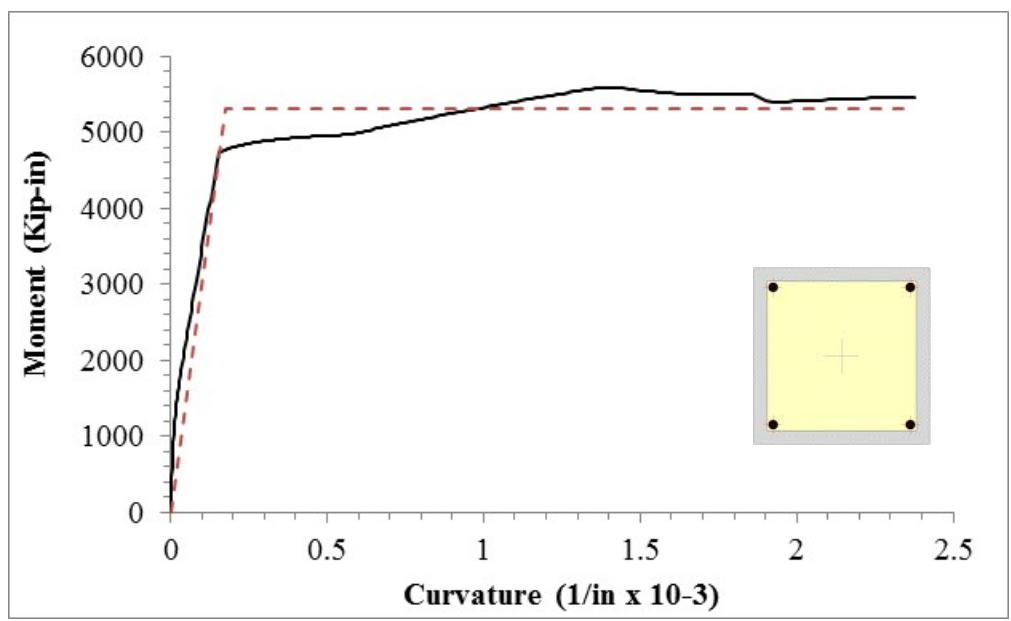

Figure G.1: Moment-curvature curve for square column 
From the moment-curvature analysis can be extracted the following data:

\section{Table G.1: Results from moment-curvature analysis}

\begin{tabular}{l|c|l}
\hline$\phi_{\mathrm{y}}$ & 0.15476 & $\left(1 /\right.$ in) $\times 10^{-3}$ \\
\hline$\phi_{\mathbf{y} \text { (idealized) }}$ & 0.17429 & $(1 /$ in $) \times 10^{-3}$ \\
\hline$\phi_{\mathrm{u}}$ & 2.376 & $(1 /$ in $) \times 10^{-3}$ \\
\hline $\mathbf{M}_{\mathbf{y}}$ & 4712.69 & Kip-in \\
\hline $\mathbf{M}_{\mathbf{u}}$ & 5472.24 & Kip-in \\
\hline $\mathbf{M}_{\mathrm{p}}$ & 5307.39 & Kip-in \\
\hline
\end{tabular}

Neutral axis:

$$
c=\frac{\varepsilon_{c u}}{\phi_{u}}=\frac{0.0109}{0.002376}=4.59 \mathrm{in}
$$

Plastic Hinge Length:

$$
\begin{gathered}
L_{p}=0.08 L+0.15 f_{y e} d_{b l} \geq 0.3 f_{y e} d_{b l} \\
L_{p}=0.08(112)+0.15(68)(1.27)=21.9 \geq 0.3(68)(1.27)=25.91 \\
\therefore L_{p}=25.91 \mathrm{in}
\end{gathered}
$$

Plastic curvature:

$$
\phi_{p}=\phi_{u}-\phi_{y}=0.0022 / \text { in }
$$

Plastic rotation:

$$
\theta_{p}=L_{p} \phi_{p}=0.057 \mathrm{rad}
$$

Yield displacement:

$$
\Delta_{y}=\frac{\phi_{y} L^{2}}{3}=0.73 \mathrm{in}
$$

Plastic displacement:

$$
\Delta_{p}=\theta_{p}\left(L-0.5 L_{p}\right)=5.65 \text { in }
$$

Ultimate displacement

$$
\Delta_{u}=\Delta_{y}+\Delta_{p}=6.38 \mathrm{in}
$$

Column displacement ductility:

$$
\mu_{d}=\frac{\Delta_{u}}{\Delta_{y}}=8
$$




\section{Shear strength}

Initial Shear strength:

$$
\begin{gathered}
V_{i}=V_{s}+V_{p}+V_{c i} \\
V_{s}=\frac{A_{v} f_{y h} D^{\prime \prime}}{s} \cot \theta=\frac{2(0.11)(68)(20)}{12} * \frac{1}{0.597}=42 \mathrm{kip} \\
\tan \theta=\left(\frac{1.6 \rho_{v} A_{e}}{\Lambda \rho_{t} A_{g}}\right)^{0.25}=\left(\frac{1.6 * \frac{2(0.11)}{21(12)} *\left(0.8 * 24^{2}\right)}{1(4 * 1.27)}\right)^{0.25}=0.6 \\
V_{p}=\operatorname{Ptan} \alpha=(160) \frac{(24-4.66)}{2 * 112}=14 \mathrm{kip} \\
V_{c i}=3.5 \sqrt{f_{c e}^{\prime}} A_{e}=3.5 \sqrt{4500}\left(0.8 * 24^{2}\right)=108 \mathrm{kip} \\
V_{i}=42+14+108=164 \mathrm{kip}
\end{gathered}
$$

Final Shear strength:

$$
\begin{gathered}
V_{f}=V_{s}+V_{p}+V_{c f} \\
V_{c f}=0.5 \sqrt{f_{c e}^{\prime}} A_{e}=0.5 \sqrt{4500}\left(0.8 * 24^{2}\right)=15 \mathrm{kip} \\
V_{f}=71 \mathrm{kip}
\end{gathered}
$$

Shear demand in flexion:

$$
\begin{gathered}
V_{m}=\frac{M_{p}}{L}=\frac{(5307)}{112}=47 \text { kip } \\
V_{m}<0.85\left(V_{f}\right)
\end{gathered}
$$

This result implies that the column rotational capacity is limited by flexure and not shear. 



\section{APPENDIX H}

ADDITIONAL EXPERIMENTAL RESULTS FROM CHAPTER 4 



\section{ADDITIONAL EXPERIMENTAL RESULTS FROM CHAPTER 4}

A few representative strain gage measurements are illustrated below. The location of the strain gages is depicted in Figure 4.8.
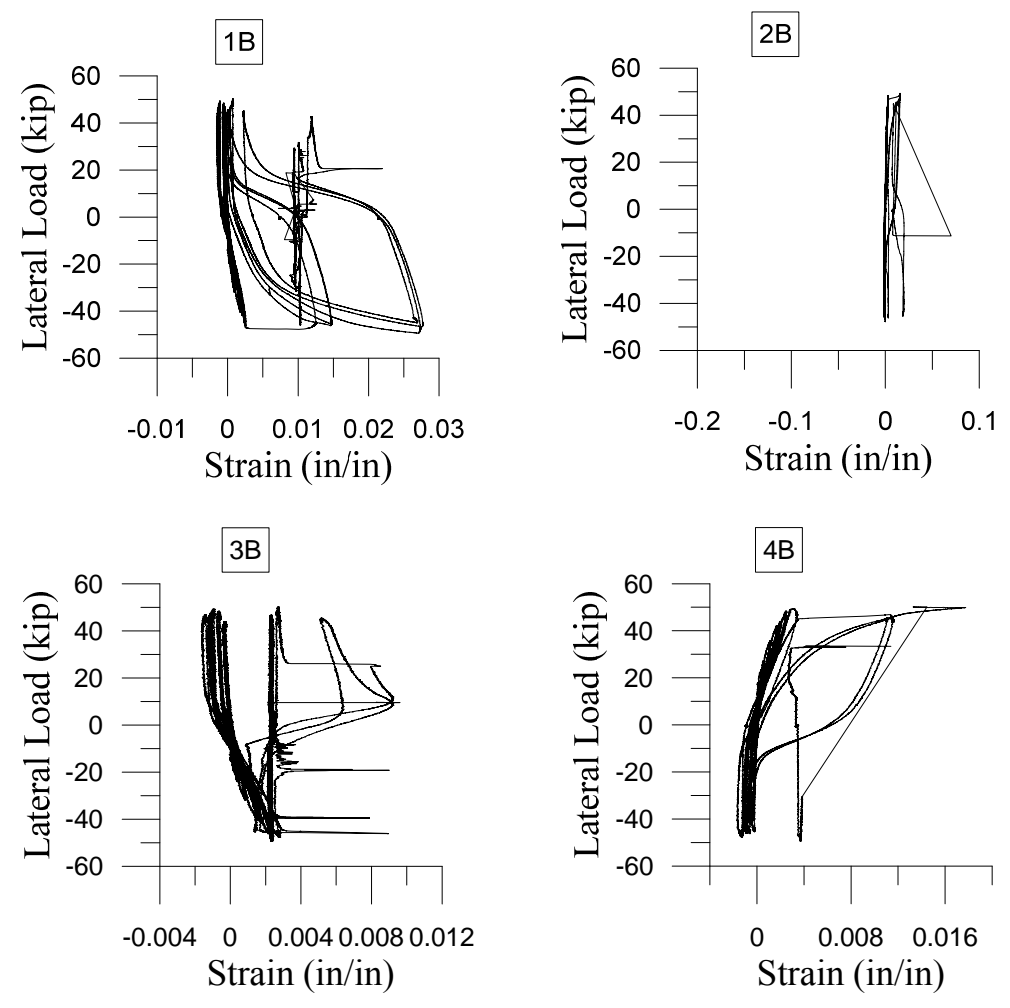

Figure H.1: Strain gage measurements in Column A-CO 
1B

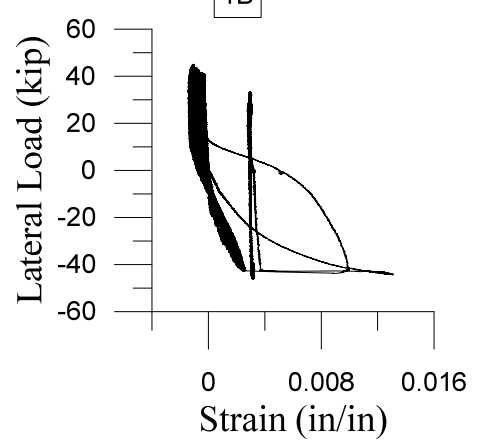

3B

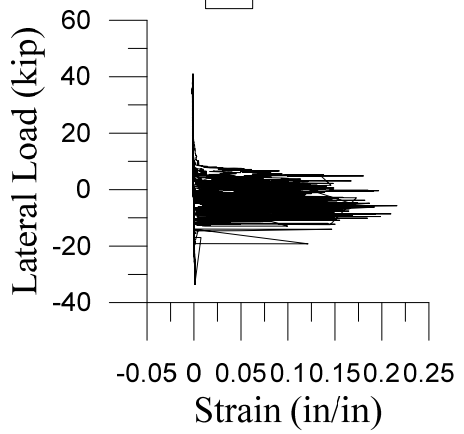

2B

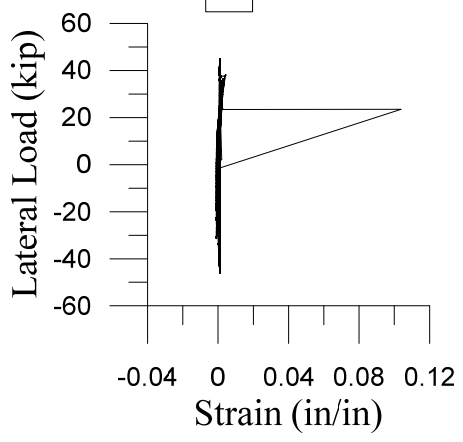

$4 B$

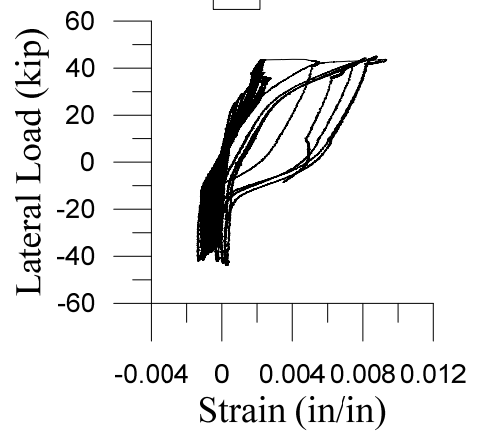

Figure H.2: Strain gage measurements in Column A-SU 


\section{APPENDIX I}

\section{DRAWINGS AND DESIGN OF CONNECTION}





\section{APPENDIX I}

\section{DRAWINGS AND DESIGN OF CONNECTION}

This appendix shows the details for the experimental program of a half-scale RC bridge bent retrofitted using Buckling Restrained Braces (BRBs).

The general layout for the retrofitted RC bent and the Buckling Restrained Braces is shown below.

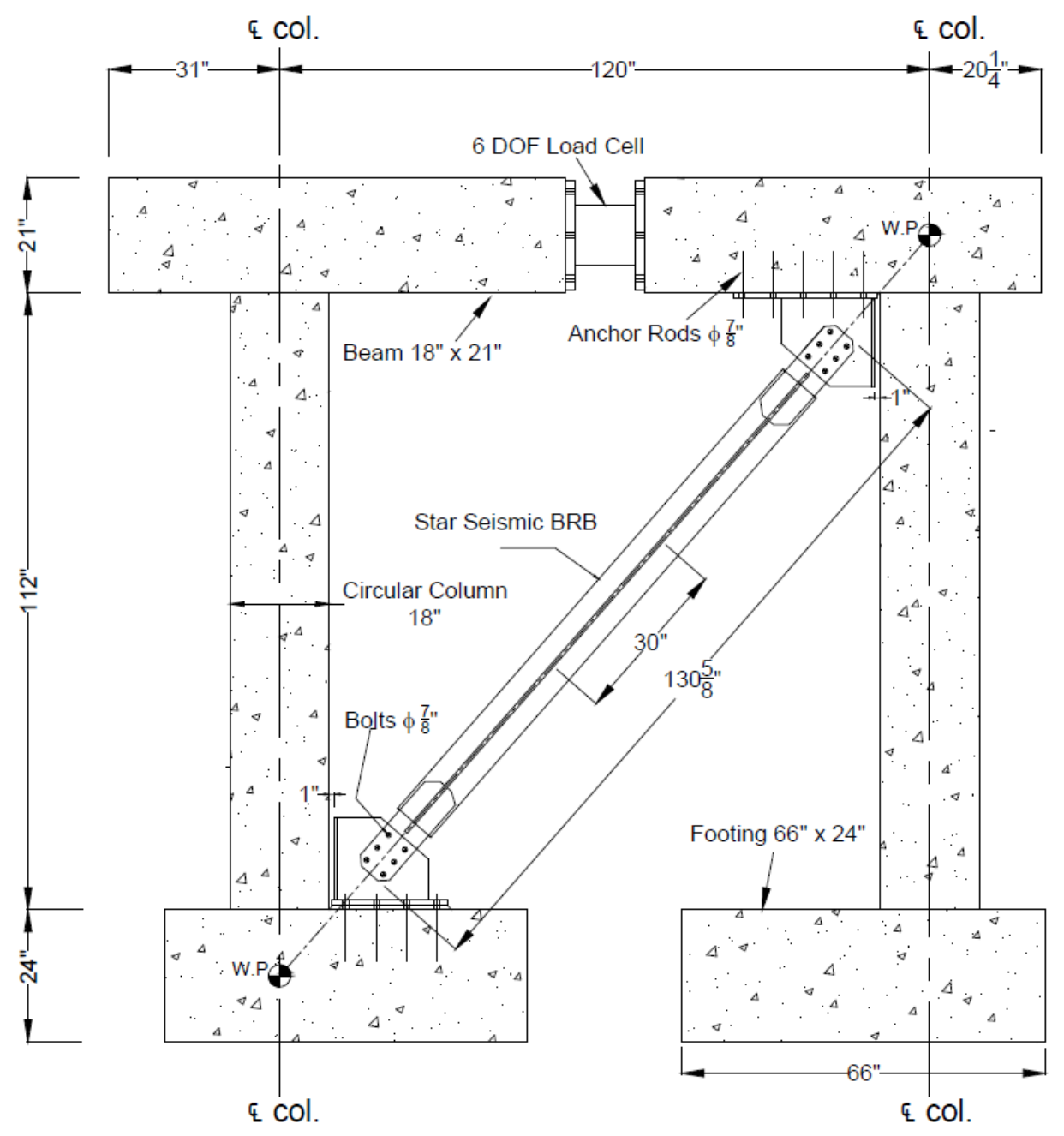

Figure I.1: Layout retrofitted bent (Model 1) 


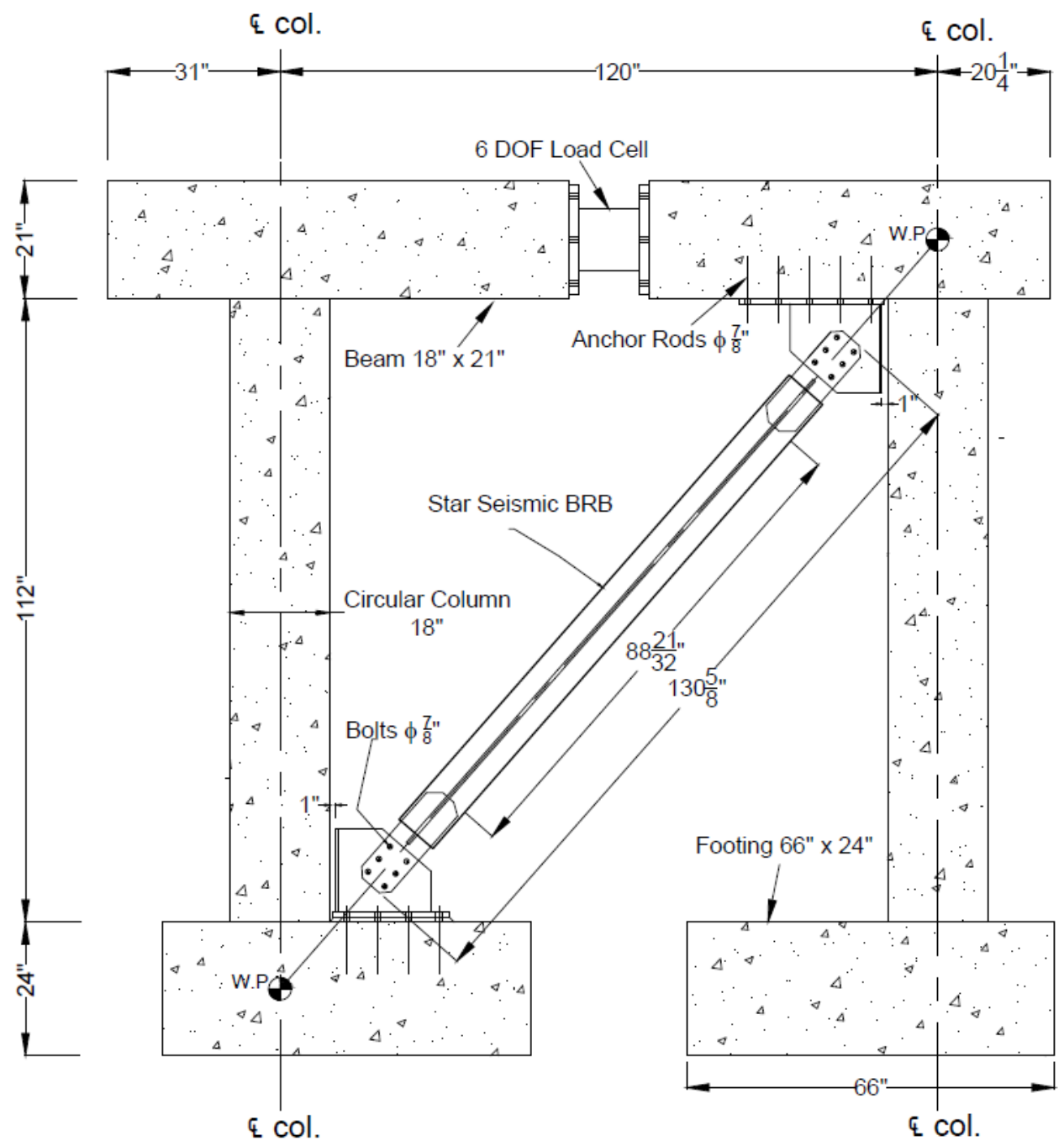

Figure I.2: Layout retrofitted bent (Model 2) 


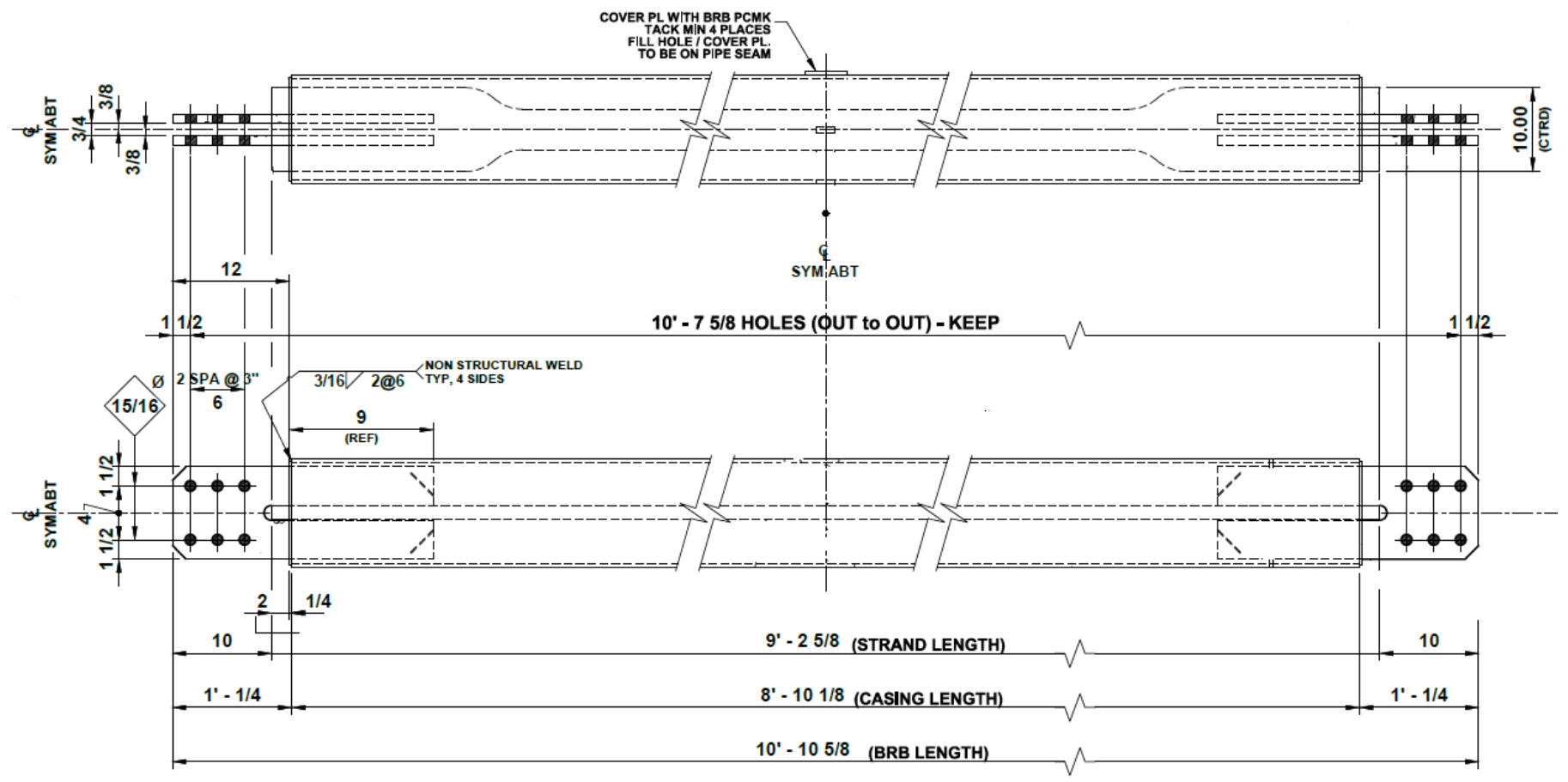

Figure I.3: Example of BRB Layout (Model 1) 


\section{Design of Top Bolted Connection}

Structural Materials:

* BRB Steel Core: ASTM A36. $F_{y}{ }^{B R B}=44.2 \mathrm{ksi}$. Coupon test required

* Gusset Plates: ASTM A572, Gr. 50. $F_{\text {ymin }}=50 \mathrm{ksi}, F_{u}=65 \mathrm{ksi}$

* Weld electrodes: E70XX

* High Strength Bolts: Group A (ASTM A325) $F_{n t}=90 \mathrm{ksi}, F_{n v}=54 \mathrm{ksi}$. Standard Holes

\section{BRB Properties:}

$F_{y B R B}:=44.2 \mathrm{ksi} \quad R_{y}:=1.1 \quad A_{B R B}:=1.2 \mathrm{in}^{2} \quad \theta_{1}:=48.74$

$E:=29000 \mathrm{ksi} \quad \beta \omega:=1.45 \quad \omega:=1.3 \quad \theta:=\theta_{1} \cdot \frac{\pi}{180}=0.851 \cdot \mathrm{rad}$

$P_{a d}:=\max \left(\beta \omega \cdot R_{y} \cdot F_{y B R B} \cdot A_{B R B}, \omega R_{y} \cdot F_{y B R B} \cdot A_{B R B}\right)$

$P_{a d}=84.599 \cdot k i p$

\section{Bolts:}

Try 7/8" bolts ASTM 325. Diameter: $d:=\frac{7}{8}$ in

Dimension of hole: $h:=d+\frac{1}{16}$ in $=0.937 \cdot$ in

Area: $A_{\text {bolt }}:=\pi \cdot \frac{d^{2}}{4}=0.601 \cdot i n^{2}$

Strength per bolt: (in double shear)

Shear:

$\phi R_{n v}:=0.75 \cdot 54 \mathrm{ksi} \cdot A_{\text {bolt }}=24.353 \cdot \mathrm{kip}$

$N_{\text {boltv }}:=\frac{P_{a d}}{\phi R_{n v} \cdot 2}=1.737$

Tension:

$F_{n t}:=90 \mathrm{ksi}$

Slip - Critical:

$\mu:=0.3$ Class A surface (the smallest slip coefficient)

$D_{u}:=1.13 \quad h_{f}:=1 \quad T_{b}:=39$ kip

AISC2011 Table J3.1: 
$n_{s}:=2 \quad R_{n s l i p}:=\mu \cdot D_{u} \cdot h_{f} \cdot T_{b} \cdot n_{s}=26.442 \mathrm{kip}$

$N_{\text {boltslip }}:=\frac{P_{a d}}{R_{\text {nslip }}}=3.199$

Try 6 bolts $7 / 8^{\prime \prime}$

$$
\phi R_{n s l i p}:=1 \cdot 6 \cdot R_{n s l i p}=158.652 \cdot k i p>\mathrm{P}_{\mathrm{ad}}
$$

Gusset Plate Design: (AISC, Steel Construction Manual 2011)

ASTM A572 Gr50 $\quad F_{y}:=50 k s i \quad F_{u}:=65 k s i$

Thickness (try): $t:=\frac{5}{8}$ in

Bearing: $s p:=3 \cdot d=2.625$ in $\quad$ use $s=3$ in $\quad s p 1:=3$ in $\quad \phi_{\text {bearing }}:=0.75$

$l_{e}:=1.5 \mathrm{in}$

$l_{\text {cedge }}:=l_{e}-\frac{h}{2}=1.031$ in $\quad l_{c}:=s p 1-h=2.062$ in

$R_{\text {nbedge }}:=1.2 l_{\text {cedge }} \cdot t \cdot F_{u}=50.273 \mathrm{kip}$

$R_{\text {nbear }}:=1.2 l_{c} \cdot t \cdot F_{u}=100.547 \mathrm{kip}$

Upper Limit: $\quad R_{\text {nupper }}:=2.4 \cdot d \cdot t \cdot F_{u}=85.313 \mathrm{kip}$

Using 6 bolts:

$\phi R_{\text {nbearing }}:=0.75 \cdot\left\{2 \cdot \min \left(R_{\text {nbedge }}, R_{\text {nupper }}\right)+4 \cdot \min \left(R_{\text {nbedge }}, R_{\text {nupper }}\right)\right\}=331.3$ kip $>\mathrm{P}_{\mathrm{ad}}$

Block shear strength:

$h_{1}:=d+\frac{1}{8}$ in $=1$ in $\quad U_{b s}:=1$ (for gusset plate)

$A_{g v}:=2 \cdot t \cdot\left(2 \cdot s p 1+l_{e}\right)=9.375 i n^{2} \quad A_{n v}:=2 \cdot t \cdot\left(2 \cdot s p 1+l_{e}-2.5 \cdot h_{1}\right)=6.25 i n^{2}$

$A_{n t}:=t \cdot\left(2 \cdot l_{e}-h_{1}\right)=1.25 \mathrm{in}^{2}$

$\phi R_{n b s}:=0.75 \cdot \min \left[\left(0.6 F_{u} A_{n v}+U_{b s} F_{u} A_{n t}\right),\left(0.6 F_{y} A_{g v}+U_{b s} F_{u} A_{n t}\right)\right]=243.75 k i p>\mathrm{P}_{\mathrm{ad}} \quad(\mathrm{OK})$

Whitmore section:

$l w:=\left(2 \cdot 2+2 \cdot 6 \cdot \tan \left(\frac{\pi}{6}\right)\right)$ in $=10.928$ in

$\underline{\text { Tension on the gross area: }}$

$A_{g}:=l w \cdot t=6.83$ in $^{2} \quad \phi P_{\text {ngross }}:=0.9 F_{y} \cdot A_{g}=307.356$ kip $>\mathrm{P}_{\text {ad }} \quad(\mathrm{OK})$ 
Tension on the net area:

$\phi P_{\text {nnet }}:=0.75 \cdot F_{u} \cdot t \cdot\left(l w-2 \cdot h_{1}\right)=272.031$ kip $>\mathrm{P}_{\mathrm{ad}} \quad(\mathrm{OK})$

Buckling Strength: (compression) $\quad$ AISC J4.4 and E3
$4.71 \cdot \sqrt{\frac{E}{F_{y}}}=113.432$
$A_{g}=6.83 \mathrm{in}^{2}$
Inertia $:=l w \cdot \frac{t^{3}}{12}=0.222 \mathrm{in}^{4}$
$r:=\sqrt{\frac{\text { Inertia }}{A_{g}}}=0.18$ in $\quad l_{b}:=9.5 \mathrm{in}$
$k:=1$
$\frac{k \cdot l_{b}}{r}=52.654$
$F_{e}:=\frac{\pi^{2} \cdot E}{\left(\frac{k \cdot l_{b}}{r}\right)^{2}}=103.236 \mathrm{ksi}$
$F_{c r}:=0.658^{\frac{F y}{F_{e}}} \cdot F_{y}=40.825 \mathrm{ksi}$
$\phi R_{n c}:=0.9 \cdot F_{c r} \cdot A_{g}=250.959$ kip $>\mathrm{P}_{\mathrm{ad}}$
$(\mathrm{OK})$

Buckling of free edge:
$L_{f g}:=0.75 \cdot \sqrt{\frac{E}{F_{y}}} \cdot t=11.289 \mathrm{in}$
(Maximum length of free edge) 


\section{Design of Anchor Rods}

The adjusted strength of the $\mathrm{BRB}\left(\mathrm{P}_{\mathrm{ad}}\right)$ with the corresponding free body diagram were utilized to design the anchor rods as depicted in Figure I-4. The uniform force method was used to design the gusset plate to concrete connection. (AISC Manual Part 13)

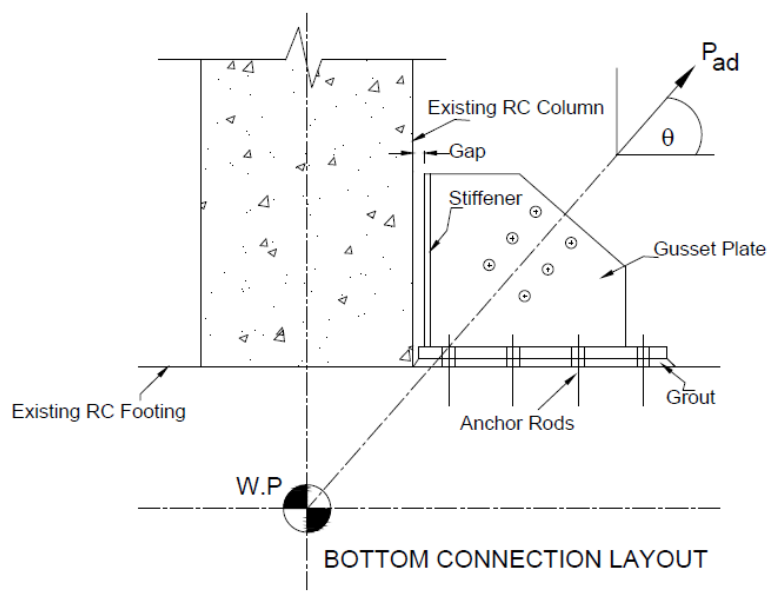

(a)

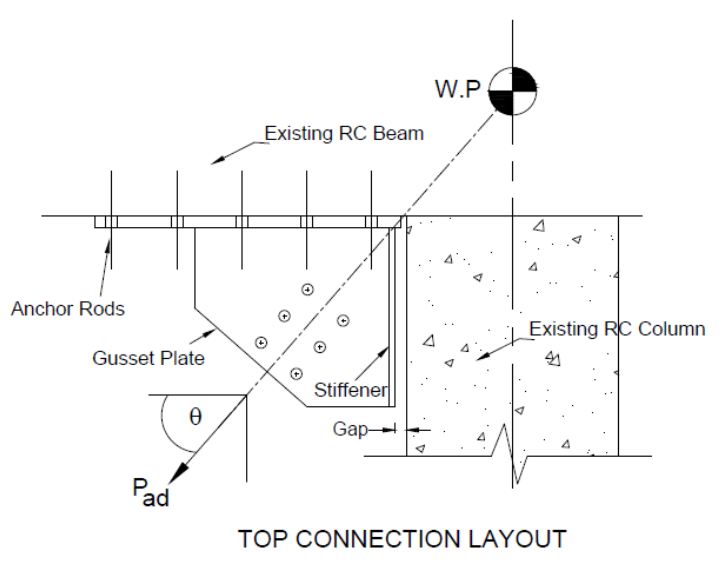

(b)

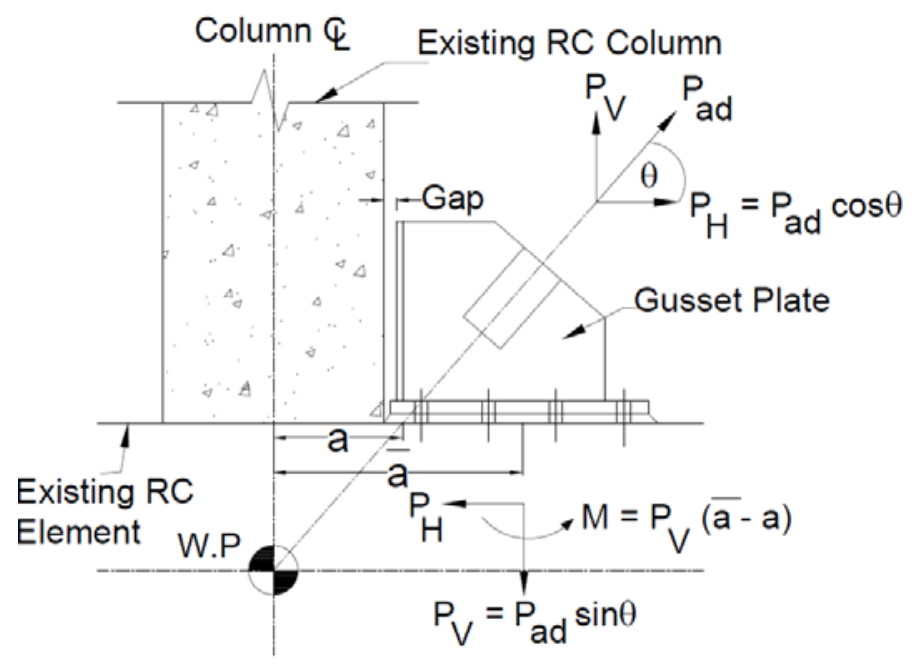

(c)

Figure I.4: Gusset Plates. (a) Bottom connection, (b) Top connection, (c) Free body diagram

The anchor rods were designed using the free software "Profis" provided by Hilti as shown in Figure I.5. The details for the connections used in the experimental program are shown in Figure I.6 and Figure I.7. 


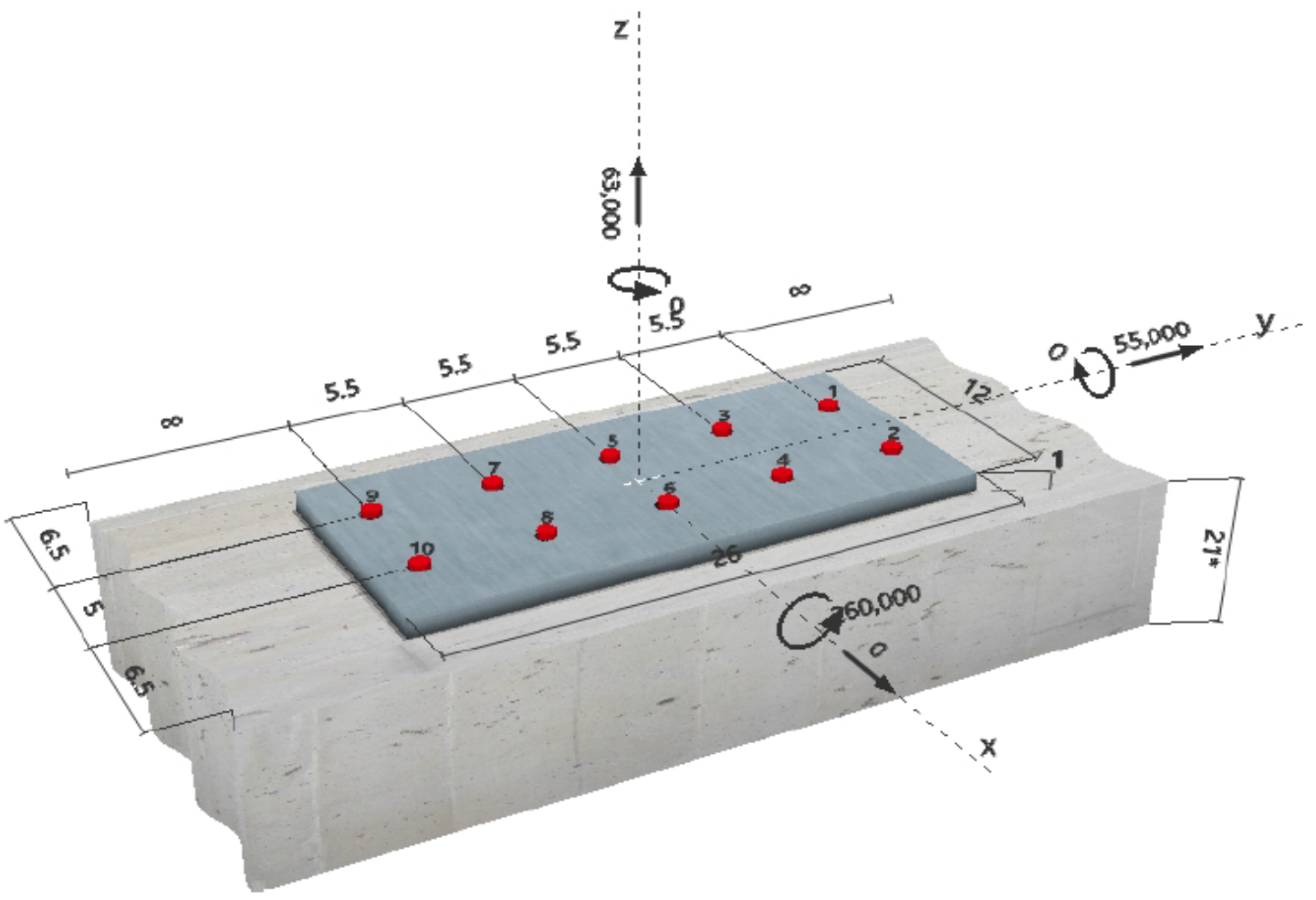

Figure I.5: Anchor rods example for top connection 

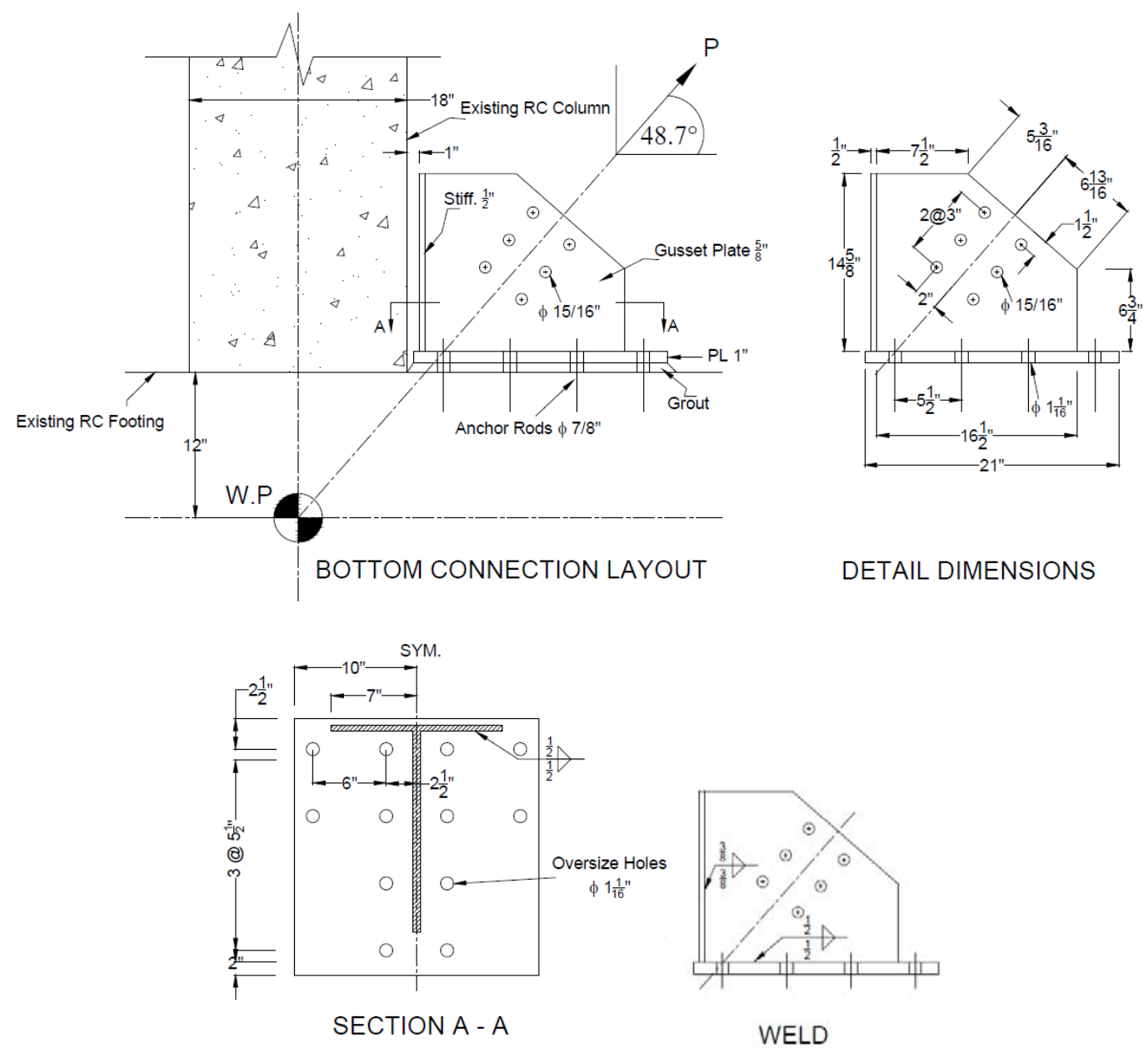

Figure I.6: Detailing of bottom gusset plate 

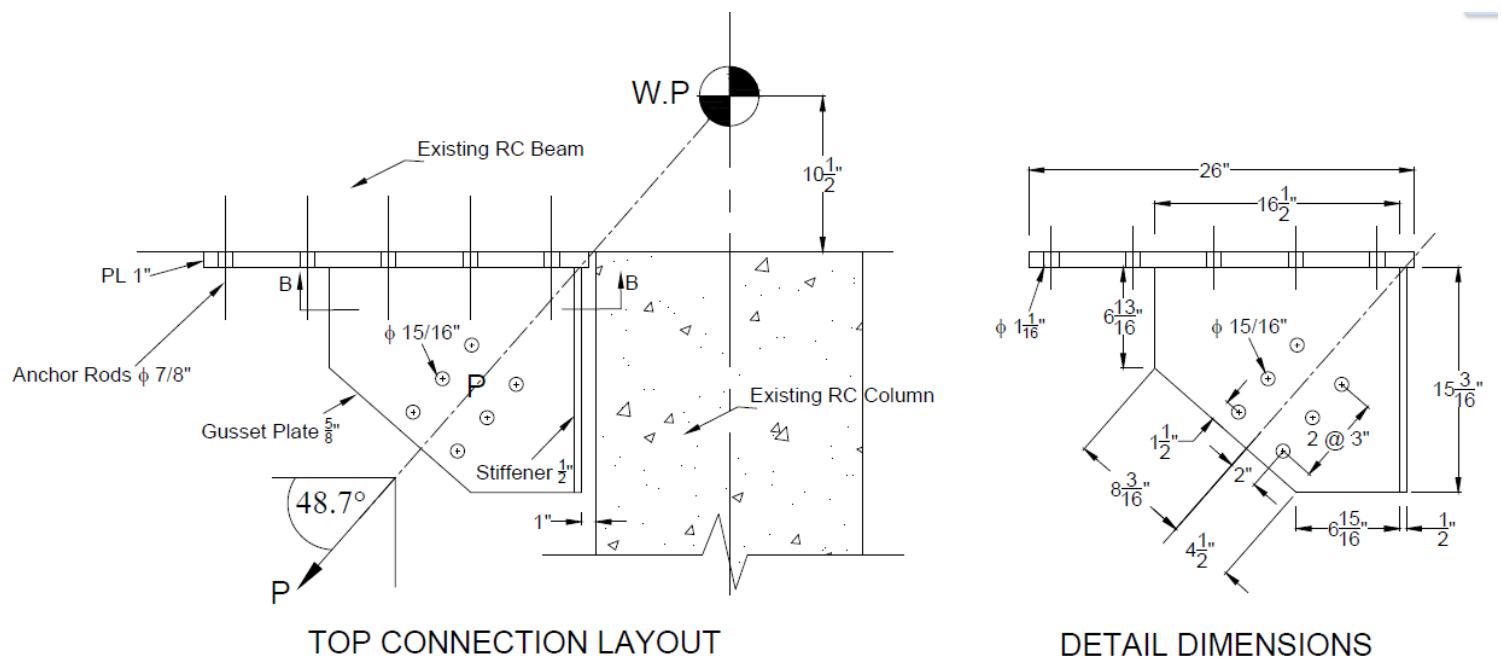

DETAIL DIMENSIONS
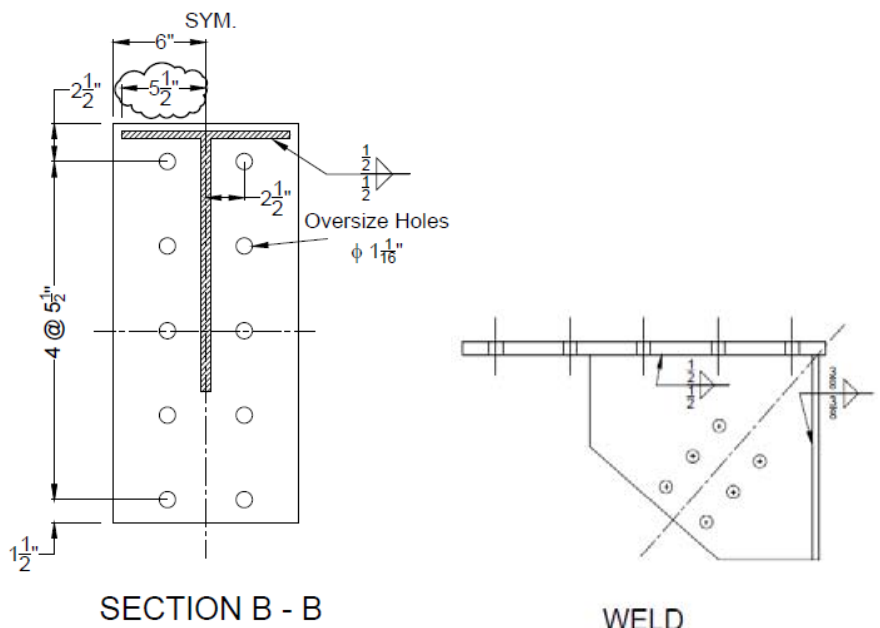

WELD

Figure I.7: Detailing of top gusset plate 


\section{APPENDIX J}

ADDITIONAL EXPERIMENTAL RESULTS FROM CHAPTER 7 



\section{APPENDIX J}

\section{ADDITIONAL EXPERIMENTAL RESULTS FROM CHAPTER 7}

More than 88 strain gage measurements were recorded in each test. For that reason, just a few representative recordings are depicted below. The figures show the lateral load vs strain. The location of the strain gages is shown in Figure 7.10. The designation for the strain gages is: component-location-height-type of reinforcement. For example F1-1-6-D means Footing 1 at location 1 (shown in Figure 7.10.), 6 inches high on dowel reinforcing steel. C2-1-4-C means Column 2 at location 1, 4 inches high on continuous steel. 

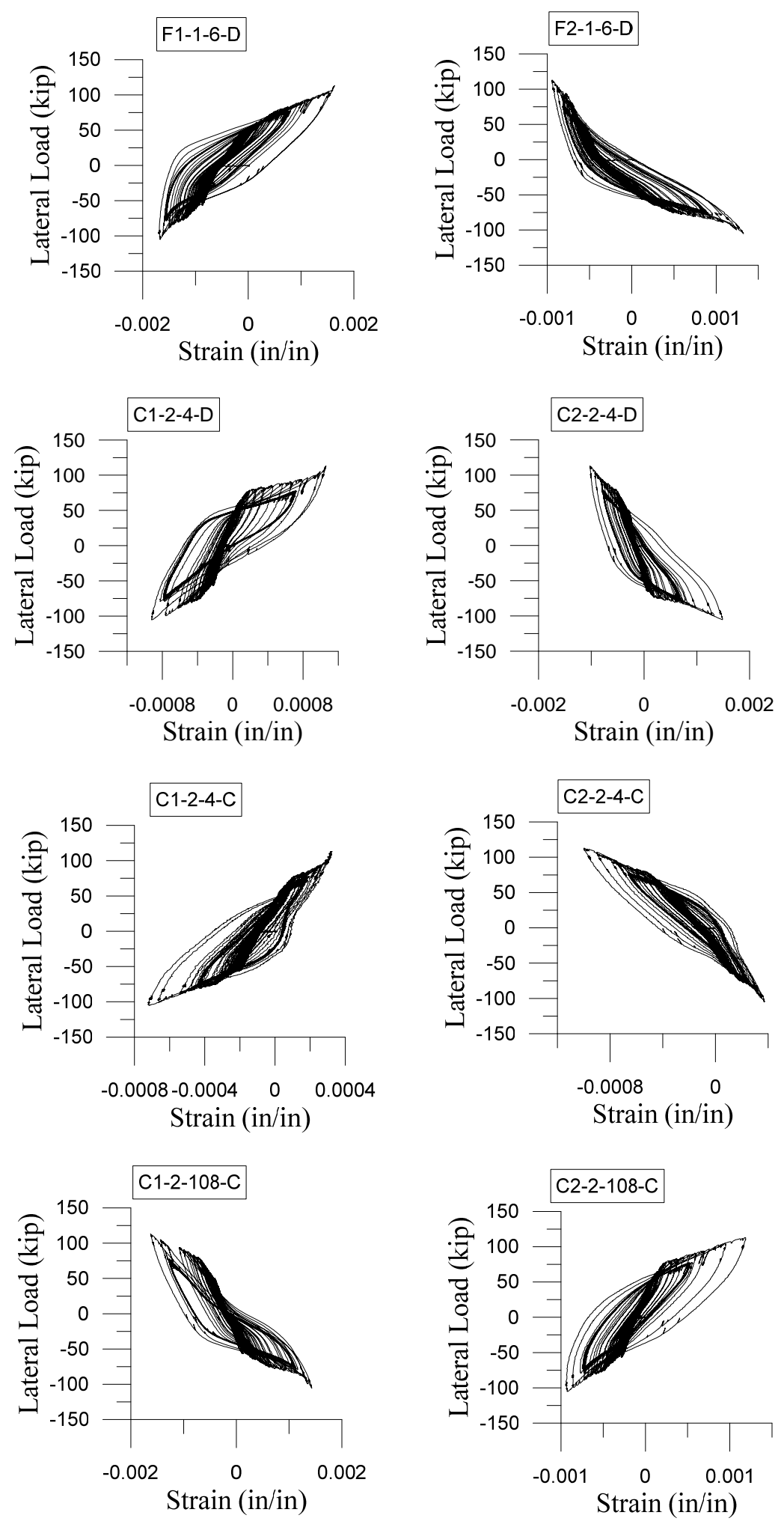

Figure J.1: Strain gage measurements (Model 1) 

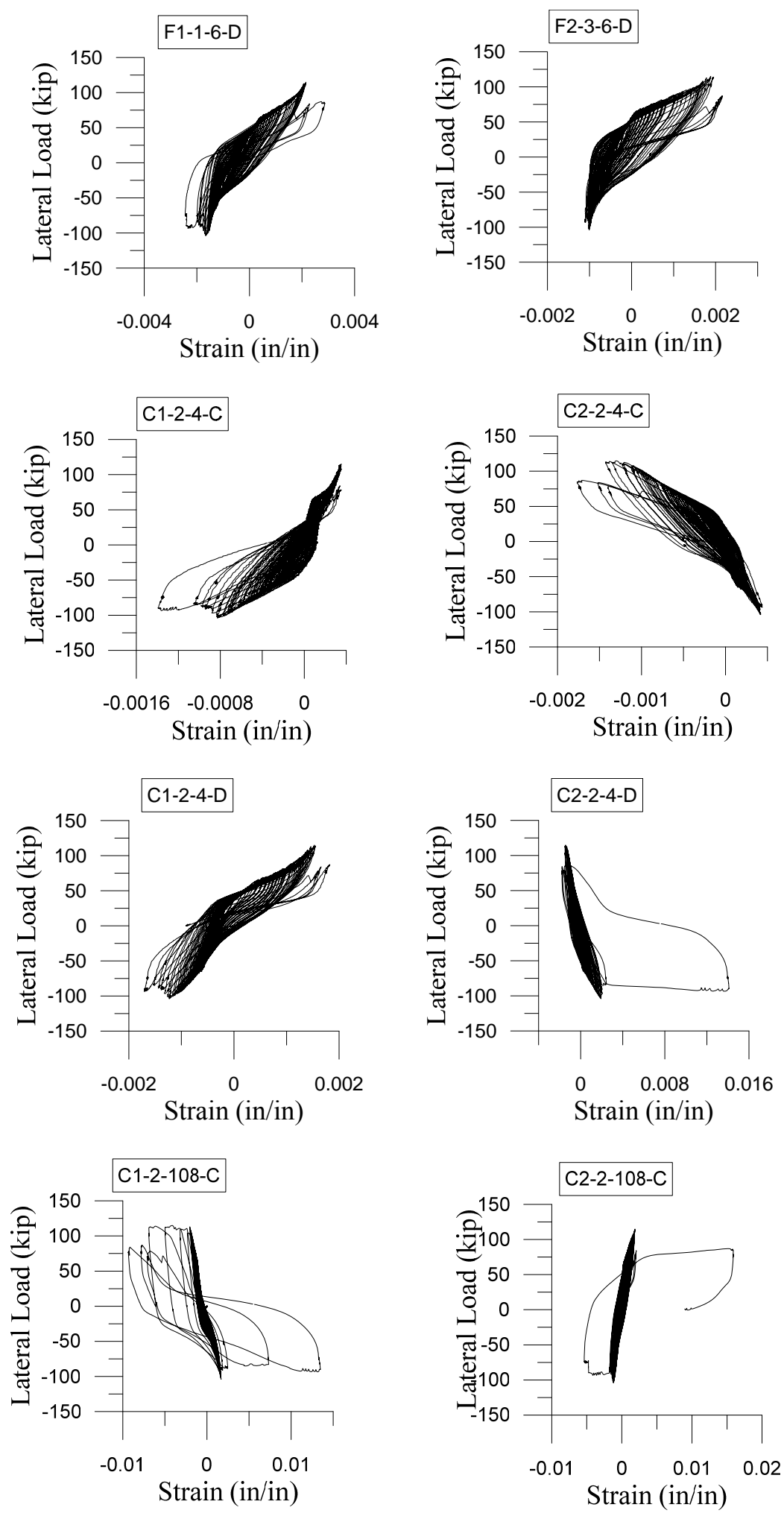

Figure J.2: Strain gage measurements (Model 2) 

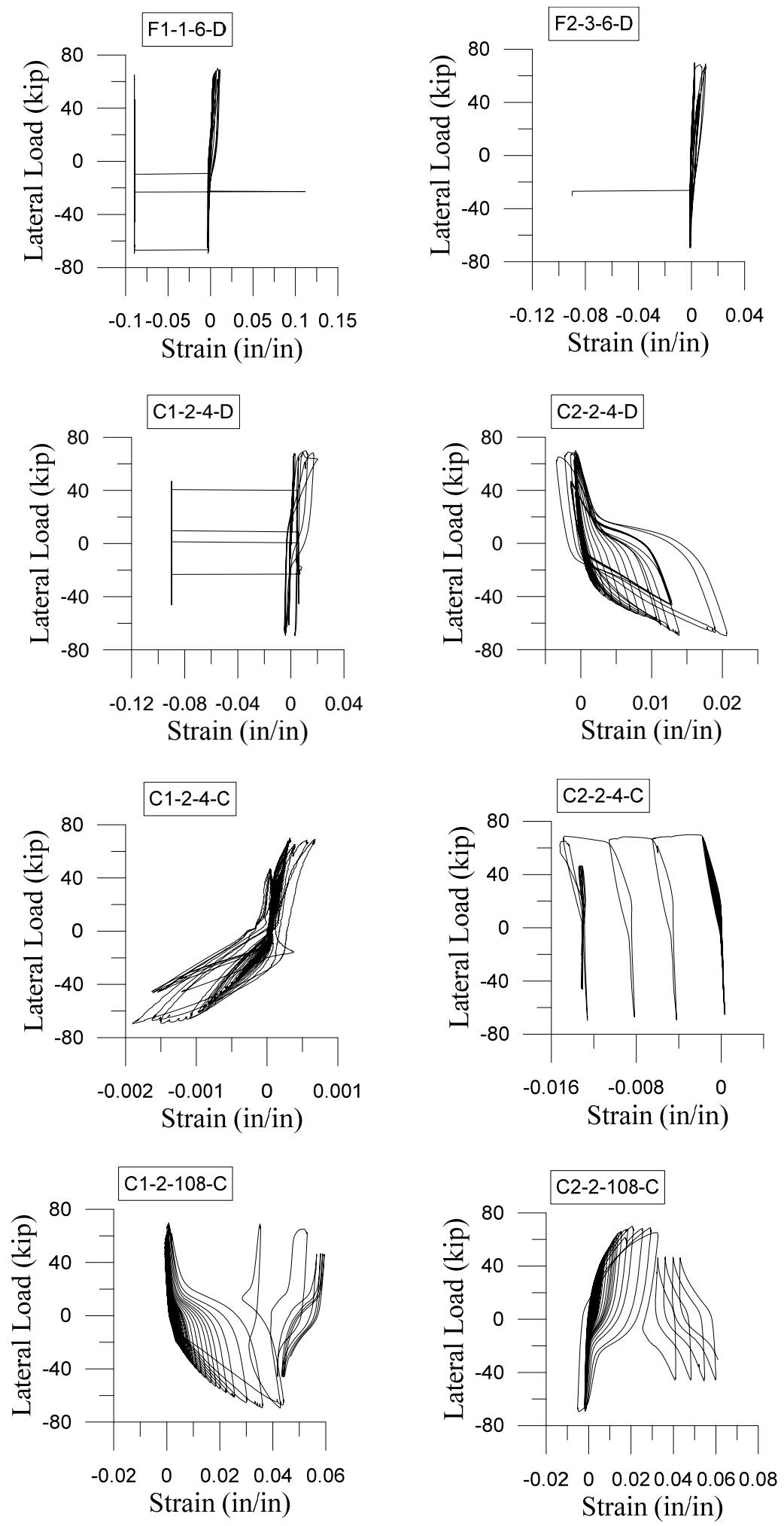

Figure J.3: Strain gage measurements (As-built bent) 